\title{
Managing Portfolio of Elective Surgical Procedures: A Multidimensional Inverse Newsvendor Problem
}

\author{
Hessam Bavafa \\ Wisconsin School of Business, University of Wisconsin-Madison, hessam.bavafa@wisc.edu \\ Charles M. Leys \\ School of Medicine and Public Health, University of Wisconsin-Madison leys@surgery.wisc.edu \\ Lerzan Örmeci \\ Department of Industrial Engineering, Koç University, lormeci@ku.edu.tr \\ Sergei Savin \\ The Wharton School, University of Pennsylvania, savin@wharton.upenn.edu
}

We consider the problem of allocating daily hospital service capacity among several types of elective surgical procedures, in the presence of random numbers of urgent procedures described by arbitrary finite-support distributions. Our focus is on the interaction between two major, constraining hospital resources: operating room (OR) and recovery bed capacity. In our model, each type of surgical procedure has an associated revenue, stochastic procedure duration, and stochastic length-of-stay (LOS). We consider arbitrary distributions of procedure and LOS durations and derive a two-moment approximation based on the Central Limit Theorem (CLT) for the total procedure duration and the daily number of occupied beds for a given portfolio of procedures. An important novel element of our model is accounting for correlation among the surgical and patient LOS durations for the procedures performed by the same surgical team. We treat the available OR and recovery bed capacity as nominal, allowing them to be exceeded at a cost. The resulting model is a novel, multi-dimensional variant of the inverse newsvendor problem, where multiple demand types compete for multiple types of service capacity.

We characterize the optimal number of elective procedures for single-specialty hospitals, and derive an optimality bound for a "front-end" capacity management approach that focuses exclusively on OR capacity. For a setting with two dominant procedure types, we provide an analytical characterization of the optimal portfolio composition under the condition that the revenue from each procedure is proportional to the expected use of hospital resources. We also derive a general analytical description of the optimal portfolio for an arbitrary number of procedure types.

For the general case of an arbitrary number of procedure types in the presence of urgent procedures, we conduct a numerical study using data we have collected at a medium-size teaching hospital. Our numerical study illustrates the composition of the optimal portfolios of elective procedures in different practical settings and investigates the quality of the CLT-based approximation and the effectiveness of the "front-end" approach to hospital capacity management. 


\section{Introduction}

Hospital care is a core component of any advanced system of health care delivery. The current changes in the way care is financed and delivered in the U.S. are forcing many hospitals to reevaluate their approaches to managing their operations and profit streams (Lee and Zenios 2012). In a study of hospital closures in the U.S. in the 1990s, Ciliberto and Lindrooth (2007) observe that low efficiency and inadequate reimbursement rates are the two main determinants of hospital closures. In settings where many hospitals aim for the highest possible utilization of every component of their service capacity, proper management of capacity allocation among various services is key to sustained profitability.

In many hospitals, surgical services are responsible for the largest share of hospital revenues and profits and have a direct impact on the utilization of "downstream" hospital resources, in particular hospital beds (Beliën et al. 2006). Given the importance of the revenue stream generated by surgical procedures, operating room (OR) capacity is often viewed as a major constraint limiting a hospital's ability to increase its revenues (see, for example, Cardoen et al. 2010 and Begen and Queyranne 2011). At the same time, limited "downstream" capacity may also play a crucial role in shaping a hospital's ability to handle a particular combination or volume of surgical procedures. For example, after each surgery, patients are transferred to hospital recovery beds, often after a stay at the post-anesthesia care unit. The time it takes a patient to recover from surgery and leave the hospital (length-of-stay, or LOS) is uncertain, and the distribution of a patient's LOS depends on the type of performed surgical procedure. The presence of two major types of constraining resources, those that are related to the surgical procedure itself and those that promote post-op recovery, can create tension between procedures that use the former resources in a more profitable manner and those that are better at using the latter. When the recovery bed capacity becomes strained, hospitals must place the patients in beds that do not match their needs, add temporary beds, or even discharge patients early and cancel future elective surgeries (Song et al. 2018, Dai and Shi 2018). All these coping mechanisms can decrease patient satisfaction, disrupt other hospital activities, affect quality of care and, ultimately, hurt a hospital's competitive position. In our analysis, we focus on the interaction between OR and hospital bed capacity. The issue of particular interest to us is the performance of the "front-end" approach to hospital capacity management that focuses exclusively on OR capacity and ignores the potential impact on the "downstream" recovery bed capacity.

Our paper makes the following contributions to the literature:

1. We develop a novel model for optimizing the daily portfolio of elective surgical procedures by considering the interaction between the utilization of two limited resources: OR and hospital 
bed capacity. In our model, each surgical procedure has an associated expected revenue, stochastic duration, as well as stochastic LOS with arbitrary finite-support distributions.

To account for the observed effects of surgical teams on the surgery outcomes (Wang et al. 2018), we consider the setting where the surgical and LOS durations for the procedures performed by the same surgical team are perfectly correlated. For given daily numbers of procedures, we use a two-moment approximation based on the Central Limit Theorem (CLT) to derive distributions for the total required OR capacity and the total required number of recovery beds (Proposition 1). We assume that, for each procedure type, the total daily number of procedures is comprised of elective procedures, and a random number of urgent procedures that the hospital must accommodate, with the number of elective procedures bounded from above. In optimizing the daily portfolio of elective procedures, we allow for the total resource requirements to exceed the nominal capacity values, with penalty costs imposed when this occurs. The resulting model is a new variant of the inverse newsvendor problem introduced in Carr and Lovejoy (2000), namely, the variant where multiple demand types compete for multiple limited resources.

2. For the single-procedure setting, we provide a complete characterization of the optimal number of elective procedures and its monotonicity properties (Proposition 2). In addition, we derive a bound on the relative performance of the "front-end" capacity allocation policy (Proposition 3).

3. In the setting with multiple types of elective procedures, we derive two separate sets of results, both in the absence of urgent procedures. First, for a setting with two dominant procedure types, we provide an analytical characterization of the optimal portfolio composition under a condition that both procedure types have associated revenues proportional to the expected resource use but are asymmetric in terms of the second moments of their resource usage (Proposition 4). For an arbitrary number of procedure types, in the absence of limitations on the elective surgeries, we offer a general analytical characterization of the optimal portfolio (Proposition B1 in the Appendix), as well as sharper expressions for the optimal portfolio values in the special case of "proportional" revenues and standard deviations for OR and recovery bed usage for all procedure types (Corollary B1 in the Appendix).

4. We validate the use of the CLT-based approximation by comparing it with the exact solution for the single-procedure setting where both surgical and LOS durations are represented by two-point distributions. For the general case with $n>2$ procedures there exist no closed-form expressions for the usage of hospital resources, and we use a numerical study based on a real-life dataset to show that the first two moments of the exact distributions are sufficient to approximate the total duration and the occupancy levels for the purposes of optimizing the portfolio of elective surgical procedures. We also investigate the composition of the optimal portfolios with the approximated and exact solutions. 
5. We use a real-life dataset to investigate the effectiveness of the "front-end" capacity allocation approach, as well as the effects of problem parameters on the resource utilizations and the composition of optimal portfolios. This dataset includes information on procedure durations, hospital compensation amounts, and patient LOS.

The normal approximation we derive facilitates the subsequent analyses of the effects of two different resource capacities on the optimal numbers of elective surgical procedures. In our analysis, we focus on establishing the optimal "capacity buffer" values for each resource in the presence of "overcapacity" costs. Both the theoretical results we present, as well as the numerical investigation we conduct using a real-life data set, generate insights on how these capacity buffers change and interact as the parameters of the problem vary. The main take-away from our analyses is the significant effect of the variability on the optimal portfolio, which can be observed in different ways. For example, in all settings, the number of type- $i$ procedures is decreasing in the variance of its resource usage, see e.g., Proposition 2 and numerical analyses in Section 6. Also, in the twoprocedure setting, Proposition 4 suggests that the optimal number of procedures of one type is increasing in the variance of the other type's resource usage. The relationship between the optimal portfolio and variability can be simpler in special cases; e.g., for $n>2$ number of procedures in a completely proportional setting, where the average benefit with respect to each resource and the ratio of variances for the OR and bed occupancies are equal for all procedures, the number of each procedure in the optimal portfolio is inversely proportional to its variance of surgery durations, or equivalently to its variance of bed occupancy (Corollary B1).

The remainder of this paper is organized as follows. In Section 2, we provide a review of the related literature. Our model is introduced in Section 3. Section 4 analyzes the case of a single procedure type, and Section 5 provides results for the settings with two types of procedures. Our numerical study is described in Section 6. Section 7 discusses our findings and outlines potential extensions to our work. Our results for an arbitrary number of surgical procedure types are given in Appendix B.

\section{Literature Review}

Our paper focuses on the optimal management of limited hospital service capacity and is related to revenue management (RM) literature. Our model considers two homogeneous resources, hospital beds and OR time, and assumes that the revenues for surgical procedures are exogenously specified, either by the government or by private insurers. Thus, we focus on quantity-based RM rather than price-based RM. Quantity-based RM has been addressed extensively in the operations management literature; see Talluri and Van Ryzin (2005) for a comprehensive review on the subject. Early papers in this literature stream, starting with the seminal work of Littlewood (2005) (first published in 
1972), focused on services with fixed durations, such as air travel. However, in many industries such as hotels, rentals, telecommunications, and health care, customers utilize service resources for varying lengths of time. Moreover, this line of literature mostly considers only a single resource.

We first consider the studies on the single-resource capacity. The earlier studies in this area mainly focused on the effects of LOS in hotel RM: for example, Weatherford (1995) evaluates the effect of including LOS in the optimization model, but treats the LOS as fixed and announced by customers in advance at the time when a hotel reservation is made. Papers that treat occupancy times as stochastic rely largely on numerical methods and provide limited analytical insights into the nature of optimal capacity allocation policies (Bitran and Mondschein 1995, Lai and Ng 2005). Later on, the "stochastic knapsack" models, with Poisson arrivals of customers and exponential service times, have been proposed to model service systems with unscheduled customer arrivals and random service durations (Altman et al. 2001, Örmeci et al. 2001, Savin et al. 2005, Örmeci and Burnetas 2005, Papier and Thonemann 2010). In the context of health care, RM problems with LOS durations have been considered more recently. Ayvaz and Huh (2010) is one of the first such studies, modeling the allocation of hospital capacity among two patient types under a fixed LOS. Helm et al. (2011) develop an MDP model to study the optimal patient admission policies and show that both the cancellations of scheduled procedures and the admissions of call-in (unscheduled non-emergency) procedures are determined by optimal thresholds on the number of occupied beds. Bekker and Koeleman (2011) use a heavy traffic approximation for the $G / G / \infty$ queue to calculate the expected daily bed occupancy for any given weekly patient admission pattern and to determine the number of daily elective procedures that minimizes the deviation of the offered load from the target load. All these studies, except for Bekker and Koeleman (2011), assume either a fixed or exponential LOS durations. Unlike these papers, our study only assumes that patient hospital stay durations have a finite support, and makes no assumptions on the shape of the distributions of these durations. Although our assumptions on the LOS durations are similar to those of Bekker and Koeleman (2011), our analysis deals with multiple hospital resources by simultaneously studying the allocation of OR and recovery bed capacity, rather than just one resource as considered in Bekker and Koeleman (2011).

One stream in health care operations literature that analyzes capacity management for multiple resources in hospitals uses mainly mathematical models. Most recently, Rath et al. (2017) propose a mathematical model that considers two resources, namely OR and anesthesiologists, to solve an OR scheduling problem. Among papers that explicitly model patient LOS, Adan and Vissers (2002) consider patients with deterministic resource requirements (beds, operating theater capacity, nursing capacity and intensive-care beds) and minimize the deviation between the realized and 
the target resource utilizations using an LP model. Adan et al. (2009) demonstrate the importance of accounting for randomness in patient LOS durations when making decisions regarding patient hospital admissions. Beliën and Demeulemeester (2007), Vanberkel et al. (2011), and Essen et al. (2013) use the sum of multinomial random variables to approximate the number of occupied beds. Helm and Van Oyen (2014) formulate a new Poisson-arrival-location model to compute the probability distributions for the number of occupied beds. In the present work, we too model the distribution of the number of occupied beds, as well as that of the surgical durations, but our analytical investigation centers on the impact of LOS variability on the structure of the optimal portfolios of surgical procedures. Thus, our approach is closer to that adopted in the areas of RM and inventory control, rather than this stream.

The other stream of literature that models multiple resources focuses on discrete-time Markov decision frameworks. Nunes et al. (2009) consider multiple patient groups with care processes that require service from multiple resources for discrete random amounts of time and develop a Markov decision process (MDP) model to determine the optimal surgery schedule that stabilizes average hospital resources utilization at desirable levels. However, they conclude that the MDP approach is not suitable for realistically-sized problem instances. More recently, Barz and Rajaram (2015) and Hulshof et al. (2016) developed MDP models for admission control of patients in the presence of multiple hospital resources, with the patient flow modeled using Markov chain dynamics. The resulting models are analyzed via the approximate dynamic programming framework. Truong et al. (2013) also consider a surgery scheduling problem that accounts for both OR and bed capacity constraints, while assuming exponential LOS durations. All these studies consider short-term, tactical control of mismatches between the demand for the hospital resources and the resource capacity. In contrast, our focus is on a medium-term, more strategic approach of selecting the optimal portfolio of surgical procedures.

In summary, we aim to determine the total daily number of elective procedures in the presence of urgent procedures that should be accepted. Our model allows the total resource requirements to exceed the nominal capacity values, with corresponding penalty costs upon such an excess. Hence, the spirit of our study is similar to the inverse newsvendor problem introduced in Carr and Lovejoy (2000). The distinguishing characteristics of our paper are as follows: to account for multiple resources with no assumption on the distribution of their random use by the patients and to provide a strategic approach to find an optimal daily portfolio of surgical procedures.

\section{Allocating Multiple Hospital Resources to Multiple Types of Surgical Procedures: A Model}

We consider a setting where hospital manages its resources by selecting the optimal daily portfolio of $n$ types of elective surgical procedures $\left(a_{1}^{e}, \ldots, a_{n}^{e}\right)$ to perform on any day $t$ in the presence 
of a random vector of urgent procedures $\left(a_{1, t}^{u}, \ldots, a_{n, t}^{u}\right)$, so that the total daily number of type- $i$ procedures on day $t$ is

$$
a_{i, t}=a_{i}^{e}+a_{i, t}^{u}, i=1, \ldots, n
$$

We focus on daily portfolios to isolate the effect of the competition between surgical procedures for different hospital resources, so that our results can identify the desired volume of surgical procedures at the strategic level. In practice, operational-level surgical plans often use a weekly scheduling cycle to incorporate the dependency of certain factors, such as surgeons' preferences, on the day of the week. In Section 7, we discuss how our model can be extended to handle these weekly cycles.

A type- $i$ procedure brings a reward of $R_{i}$. Following the common financial compensation rules used by the U.S. government (Medicare) as well as private insurers, we assume that this reward does not depend on the LOS of a patient. However, our model is still valid in the settings when a hospital charges the patients depending on the duration of their LOS. Since the LOS of a given patient cannot be known in advance, the hospital has to decide on the surgery mix using the LOS distributions of procedure types rather than the actual realizations of LOS.

We also assume that the hospital receives the same compensation irrespective of whether the procedure is elective or nonelective/urgent. Note that some studies indicate that urgent procedures may be less profitable for the hospital, see Haider et al. (2015). However, since in our setting the hospital must accept all urgent procedures, differentiating the rewards with respect to urgency will not affect the optimal mix of elective surgeries.

All surgical patients require access to multiple hospital resources, with ORs and recovery beds being the major ones. Although our model and analysis can be extended to any number of resources, the model presented below explicitly accounts for the two major resources with limited nominal capacities: operating rooms are available for a total amount of $\tau$ time units each day and there are $c$ hospital beds.

The nominal OR capacity, $\tau$, reflects the strategic decision of the hospital, and may depend on several factors such as the number of ORs, the number of surgeons using the ORs, the number of OR staff and their daily work schedules. The actual daily use of OR fluctuates around this nominal capacity level due to several factors, including random surgery durations. One of our aims is to understand the effects of such fluctuations on managing hospital resources. We assume that the hospital incurs a penalty cost of $\$ \pi^{S}$ per hour when the total duration of all surgical procedures performed on a particular day exceeds $\tau$. The actual corresponding penalty cost may change with the type of the patient who causes the OR capacity violation, with this type being a random variable that depends on the operational level activities such as daily scheduling policies and arrival 
processes of urgent procedures during the day. We assume a generic penalty cost, $\$ \pi^{S}$, and interpret it as the expected hourly value of these random OR capacity violation costs. Our numerical study (Section 6) shows how to estimate $\$ \pi^{S}$ from hospital data. We also perform sensitivity analysis that explores the effect of this cost parameter on the optimal daily portfolio of elective procedures and the expected utilization of OR and bed capacities.

Hospitals account for beds either as the number of physical beds or as the number of operational or staffed beds, where the former is generally greater than the latter, see De Bruin et al. (2010). The nominal number of beds in our model, $c$, corresponds to the number of operational beds, so that the hospital management plans to staff $c$ beds at the strategic level, and keeps some spare beds to be staffed only when needed. We assume that a hospital incurs a penalty cost of $\$ \pi^{N}$ for every patient in excess of $c$. The actual cost may depend on the type of the patient that causes the bed capacity violation, however, as in the case of operating room, we interpret $\$ \pi^{N}$ as the expected value of this actual cost. Section 6 provides an estimation example and a sensitivity analysis for this cost as well.

We should note that the capacity values we use in our model should be considered as "nominal," i.e., the values that can be exceeded, if necessary, at a cost. Such "nominal" values can be contrasted to much higher maximum capacity determined, for example, by the maximum possible duration of all surgical procedures over all ORs or by the maximum possible number of staffed beds the hospital can operate at any given time. In actual hospital environments, the resource utilization that approaches the levels defined by this "hard" capacity constraints is cost prohibitive, and hospitals typically operate under moderate expected utilization of maximum resource capacity levels, e.g., around $75 \%$ for the operating room utilization (Foster 2012) and around 63\% for the bed utilization (National Center for Health Statistics 2017). In our model, we incorporate this observation using penalty costs associated with exceeding the nominal capacity, with high penalty costs keeping the optimal resource utilization well below the "hard" resource constraints.

Our aim is to characterize the patient mix that maximizes the expected profit of the hospital given the capacity of the resources at a strategic level. For many hospitals, it may not be possible to generate as many surgery requests as the optimal solution would suggest. To account for such restrictions on the demand for elective surgeries, we impose daily caps on the numbers of surgical procedures so that:

$$
a_{i}^{e} \leq U_{i}, \quad i \in\{1,2, \ldots, n\} .
$$

Finally, for analytical tractability, we treat all numbers of surgical procedures as continuous variables. To summarize, our model focuses on the optimal daily portfolio of $n$ types of elective surgical procedures $\left(a_{1}^{e}, \ldots, a_{n}^{e}\right)$ to be performed on any day $t$ satisfying $a_{i}^{e} \leq U_{i}$ for all $i$ in the presence of a random vector of urgent procedures $\left(a_{1, t}^{u}, \ldots, a_{n, t}^{u}\right)$ with the objective of maximizing the hospital's daily expected profit. 


\subsection{Random components: Durations of surgery and LOS, number of urgent procedures}

We use $S_{i, j, t}$ and $L_{i, j, t}$ to denote the random variables describing the duration of the $j$-th procedure of type $i$ on day $t(t=1,2, \ldots)$, and the length of stay (LOS) duration for a patient who underwent that procedure, respectively. We assume that both the surgical duration $\left(S_{i, j, t}\right)$ and LOS $\left(L_{i, j, t}\right)$ are bounded, which is valid for all procedures when reasonably high upper bounds are chosen. These random variables are related to each other through a number of factors. For example, studies that classify surgical complications show that the LOS of a patient increases with the level of surgical complications, see e.g., Dindo et al. (2004), and such complications would also affect the surgical duration. Accordingly, we define a joint probability distribution for the pairs $\left(S_{i, j, t}, L_{i, j, t}\right)$.

Assumption 1. $S_{i, j, t}$ and $L_{i, j, t}$ are distributed according to a joint continuous probability distribution with a finite support, and both the support and the probability density function (pdf) of the distribution depend only on $i$, but not on $j$ or $t$ :

$$
\begin{aligned}
p\left(S_{i, j, t}=s, L_{i, j, t}=l\right)=f_{i}(s, l), \quad & s \in\left[0, S_{\max }^{i}\right], l \in\left[0, L_{\max }^{i}\right] \\
& i=1, \ldots, n, j=1, \ldots, a_{i, t}, t=1,2, \ldots
\end{aligned}
$$

where $0<S_{\max }^{i}$ and $0<L_{\max }^{i}$, and

$$
\int_{0}^{L_{\max }^{i}} \int_{0}^{S_{\max }^{i}} f_{i}(s, l) d s d l=1, \quad i=1, \ldots, n .
$$

Next, we assume that the outcomes of surgeries performed on different days, as well as of those from different types, are independent of each other. These assumptions are rather natural, and they facilitate the subsequent mathematical analyses.

Assumption 2. The duration pairs $\left(S_{i_{1}, j_{1}, t_{1}}, L_{i_{2}, j_{2}, t_{1}}\right)$ and $\left(S_{i_{1}, j_{1}, t_{2}}, L_{i_{2}, j_{2}, t_{2}}\right)$ for all procedures performed on different days $t_{1} \neq t_{2}$ are independent random variables.

ASSUMPTION 3. The duration pairs $\left(S_{i_{1}, j_{1}, t}, L_{i_{1}, j_{1}, t}\right)$ and $\left(S_{i_{2}, j_{2}, t}, L_{i_{2}, j_{2}, t}\right)$ for all procedures of different types $\left(i_{1} \neq i_{2}\right)$ performed on the same day $t$ are independent random variables.

The following parameters defined for each procedure type will be useful in the subsequent analyses. More specifically, we let

$$
\mu_{i}^{S}=\int_{0}^{S_{\max }^{i}} s f_{i}^{S}(s) d s=\int_{0}^{S_{\max }^{i}} \int_{0}^{L_{\max }^{i}} s f_{i}(s, l) d s d l,
$$

and

$$
\left(\sigma_{i}^{S}\right)^{2}=\int_{0}^{S_{\max }^{i}} f_{i}^{S}(s)\left(s-\mu_{i}^{S}\right)^{2} d s=\int_{0}^{S_{\max }^{i}} \int_{0}^{L_{\max }^{i}}\left(s-\mu_{i}^{S}\right)^{2} f_{i}(s, l) d s d l
$$


denote the expected value and the variance of the duration of a procedure of the $i$-th type. We also define the following quantities that will be used to calculate the mean and variance of the daily bed occupancy:

$$
\begin{aligned}
\mu_{i}^{N} & =\int_{0}^{L_{\max }^{i}} \int_{0}^{S_{\max }^{i}} l f_{i}(s, l) d s d l=\sum_{T=0}^{L_{\max }^{i}} p_{i, T}^{L}, \\
\left(\sigma_{i}^{N}\right)^{2} & =\sum_{T=0}^{L_{\max }^{i}} p_{i, T}^{L}\left(1-p_{i, T}^{L}\right),
\end{aligned}
$$

where

$$
p_{i, T}^{L}=\int_{T}^{L_{\max }^{i}} \int_{0}^{S_{\max }^{i}} f_{i}(s, l) d s d l, \quad T=0, \cdots, L_{\max }^{i} .
$$

Note that $p_{i, T}^{L}$ is the probability that a type- $i$ patient stays at the hospital for at least $T$ days. The relation between these quantities and the daily bed occupancy levels is explicitly established in the proof of Proposition 1, which can be found in the Appendix. Also, while patients are admitted and discharged during the day, we count the bed occupancy using midnight census. For example, a patient that is admitted any time on a particular day and released the day after is assumed to have a one-day LOS.

To account for the well-known dependence of the surgical durations on the surgical team who perform the surgery (see, e.g., Wang et al. 2018), we assume the following correlation structure for the durations associated with the procedures of the same type $i$ performed on the same day $t$. Consider a partition of the set of procedures of type $i$ into $K_{i}$ subsets, with $q_{i k} \in[0,1], i=1, \ldots, n$, $k=1, \ldots, K_{i}$ being a fraction of $a_{i, t}$ procedures that belong to a subset $C_{i, k}$, with $\sum_{k=1}^{K_{i}} q_{i k}=1$. In this setting, each subset $k=1, \ldots, K_{i}$ contains the surgical procedures of type $i$ performed by the same surgeon or the same surgical team (indexed by $k=1, \ldots, K_{i}$ ). We assume that the procedures carried on by a certain surgical team on a given day result in perfectly correlated surgery and LOS duration pairs. Naturally, this perfect correlation structure is a simplification of reality; however, making this assumption produces a higher fidelity model than assuming a complete independence of surgical and LOS duration pairs resulting from procedures performed by the same surgical team. This assumption not only accounts for the effect of the surgical teams, but also implies a particular structure of the limiting distributions of the OR time and recovery bed requirements, as specified in Proposition 1 below.

Assumption 4. The duration pairs associated with procedures of type $i=1, \ldots, n$ that belong to different subsets $k_{1} \in\left\{1,2, \ldots, K_{i}\right\}$ and $k_{2} \in\left\{1,2, \ldots, K_{i}\right\}, k_{1} \neq k_{2}$, and performed on day $t$, $\left(S_{i, j_{1}, t}, L_{i, j_{1}, t}\right)$ and $\left(S_{i, j_{2}, t}, L_{i, j_{2}, t}\right), j_{1} \in C_{i, k_{1}}, j_{2} \in C_{i, k_{2}}$ are independent random variables. 
Assumption 5. All procedures of type $i=1, \ldots, n$ in a subset $k=1, \ldots, K_{i}$, performed on day $t$, result in perfectly correlated pairs of procedure and LOS durations $\left(S_{i, j_{1}, t}, L_{i, j_{1}, t}\right)$ and $\left(S_{i, j_{2}, t}, L_{i, j_{2}, t}\right)$, $j_{1}, j_{2} \in C_{i, k}$.

In order to account for this correlation structure, we define the following parameters:

$$
\begin{aligned}
& \bar{\sigma}_{i}^{S}=\sigma_{i}^{S}\left(\sqrt{\sum_{k=1}^{K_{i}} q_{i k}^{2}}\right), \\
& \bar{\sigma}_{i}^{N}=\sigma_{i}^{N}\left(\sqrt{\sum_{k=1}^{K_{i}} q_{i k}^{2}}\right), i=1, \ldots, n .
\end{aligned}
$$

Finally, we characterize the distribution of the daily numbers of urgent surgical procedures. We use $a_{i, t}^{u}$ to denote the number of type- $i$ urgent procedures on day $t$ and assume that $a_{i, t}^{u}$ 's, for all procedure types, are drawn from a joint probability distribution with a finite support.

Assumption 6. The numbers of urgent procedures on any day $t,\left(a_{1, t}^{u}, \ldots, a_{n, t}^{u}\right)$, are distributed on the interval $\left[l_{1}, h_{1}\right] \otimes \ldots \otimes\left[l_{n}, h_{n}\right]$ according to the joint $p d f f^{u}\left(\xi_{1}, \ldots, \xi_{n}\right)$. Moreover, $\left(a_{1, t}^{u}, \ldots, a_{n, t}^{u}\right)$ are distributed independently from $S_{i, j, t}$ and $L_{i, j, t}$ for any $(i, j, t)$ and independently from $\left(a_{1, t^{\prime}}^{u}, \ldots, a_{n, t^{\prime}}^{u}\right)$ for any $t^{\prime} \neq t$.

The expected number of type- $i$ urgent procedures, denoted by $\bar{\xi}_{i}$, is given by:

$$
\bar{\xi}_{i}=\int_{l_{1}}^{h_{1}} \ldots \int_{l_{n}}^{h_{n}} d \xi_{1} \ldots d \xi_{n} f^{u}\left(\xi_{1}, \ldots, \xi_{n}\right) \xi_{i} .
$$

\subsection{CLT-based approximation for the expected profit function}

We consider the setting where the same number of elective procedures is performed on any day $t$, and use the following notation for the vector of the number of elective procedures:

$$
\mathbf{a}^{e}=\left(a_{1}^{e}, \ldots, a_{n}^{e}\right)
$$

We label the vector of the urgent number of procedures performed on day $t$ as:

$$
\mathbf{a}_{t}^{u}=\left(a_{1, t}^{u}, \ldots, a_{n, t}^{u}\right)
$$

and let

$$
D_{t}\left(\mathbf{a}^{e}, \mathbf{a}_{t}^{u}\right)
$$

denote the total duration of all surgical procedures, both elective and urgent, performed on day $t$. 
In addition, we define the maximum value of LOS over all patient types by:

$$
L_{\max }=\max _{i=1, \ldots, n}\left(L_{\max }^{i}\right)
$$

In the presence of random numbers of urgent procedures, the bed occupancy on any day may, in general, be a function of the total number of procedures performed on any of the previous $L_{\max }$ days. Accordingly, we use

$$
O_{t}\left(\mathbf{a}^{e}, \mathbf{a}_{t-L_{\max }}^{u}, \ldots, \mathbf{a}_{t}^{u}\right)
$$

to denote the bed occupancy on day $t$.

We assume that the hospital aims to maximize its expected daily profit, which depends not only on the number of surgeries on day $t$, but also on the numbers of procedures performed on days $t-L_{\max }, t-L_{\max }+1, \ldots, t$. Hence, the hospital's objective must be calculated by taking the expectation with respect to the numbers of urgent procedures $a_{i, T}^{u}, i=1, \ldots, n$, over the days $T=t-L_{\max }, \ldots, t$.

Below, we use the notation $\xi_{i, T}$ to denote the random number of urgent procedures of type $i$ performed on day $T$, and $\boldsymbol{\xi}_{T}$ to denote the vector of urgent procedures of all types $\left(\xi_{1, T}, \ldots, \xi_{n, T}\right)$, performed on day $T$. Then, the expected daily profit for the hospital on any day $t$ is given by:

$$
\begin{aligned}
& \tilde{\Pi}\left(\mathbf{a}^{e}\right)=\int_{l_{1}}^{h_{1}} \ldots \int_{l_{n}}^{h_{n}} d \boldsymbol{\xi}_{t-L_{\max }} f^{u}\left(\boldsymbol{\xi}_{t-L_{\max }}\right) \cdots \int_{l_{1}}^{h_{1}} \ldots \int_{l_{n}}^{h_{n}} d \boldsymbol{\xi}_{t} f^{u}\left(\boldsymbol{\xi}_{t}\right) \\
& \left(\sum_{i=1}^{n} R_{i}\left(a_{i}^{e}+\xi_{i, t}\right)-\pi^{N} E\left[\left(O_{t}\left(\mathbf{a}^{e}, \boldsymbol{\xi}_{t-L_{\max }}, \ldots, \boldsymbol{\xi}_{t}\right)-c\right)^{+}\right]-\pi^{S} E\left[\left(D_{t}\left(\mathbf{a}^{e}, \boldsymbol{\xi}_{t}\right)-\tau\right)^{+}\right]\right),
\end{aligned}
$$

where $c$ and $\tau$ are the "nominal" values of hospital bed and daily OR capacities, respectively. In particular, (18) implies that a hospital incurs the penalty cost of $\$ \pi^{N}$ per patient when the midnight census exceeds $c$, and the penalty cost of $\$ \pi^{S}$ per hour when the total duration of all surgical procedures performed on a particular day exceeds $\tau$. We can re-write (18) as follows:

$$
\begin{aligned}
& \tilde{\Pi}\left(\mathbf{a}^{e}\right)=\sum_{i=1}^{n} R_{i}\left(a_{i}^{e}+\bar{\xi}_{i}\right)-\pi^{S} \int_{l_{1}}^{h_{1}} \ldots \int_{l_{n}}^{h_{n}} d \boldsymbol{\xi}_{t} f^{u}\left(\boldsymbol{\xi}_{t}\right) E\left[\left(D_{t}\left(\mathbf{a}^{e}, \boldsymbol{\xi}_{t}\right)-\tau\right)^{+}\right] \\
& -\pi^{N} \int_{l_{1}}^{h_{1}} \ldots \int_{l_{n}}^{h_{n}} d \boldsymbol{\xi}_{t-L_{\max }} f^{u}\left(\boldsymbol{\xi}_{t-L_{\max }}\right) \ldots \int_{l_{1}}^{h_{1}} \ldots \int_{l_{n}}^{h_{n}} d \boldsymbol{\xi}_{t} f^{u}\left(\boldsymbol{\xi}_{t}\right) E\left[\left(O_{t}\left(\mathbf{a}^{e}, \boldsymbol{\xi}_{t-L_{\max }}, \ldots, \boldsymbol{\xi}_{t}\right)-c\right)^{+}\right] .
\end{aligned}
$$

For general surgery and LOS duration distributions, it is very difficult to compute the expectations inside the integrals. Hence, we resort deriving limiting distributions for $D_{t}\left(\mathbf{a}^{e}, \mathbf{a}_{t}^{u}\right)$ and $O_{t}\left(\mathbf{a}^{e}, \mathbf{a}_{t-L_{\max }^{u}}^{u}, \ldots, \mathbf{a}_{t}^{u}\right)$ under the correlation structure defined above: 
Proposition 1. Let $\left(\mathbf{a}^{e}, \mathbf{a}_{t-L_{\max }}^{u}, \ldots, \mathbf{a}_{t}^{u}\right)$ be the vector of the numbers of procedures performed on days $t-L_{\max }, \ldots, t$, and define

$$
\begin{aligned}
M^{S}\left(\mathbf{a}^{e}, \mathbf{a}_{t}^{u}\right) & =\sum_{i=1}^{n}\left(a_{i}^{e}+a_{i, t}^{u}\right) \mu_{i}^{S}, \\
\left(\Sigma^{S}\left(\mathbf{a}^{e}, \mathbf{a}_{t}^{u}\right)\right)^{2} & =\sum_{i=1}^{n}\left(a_{i}^{e}+a_{i, t}^{u}\right)^{2}\left(\bar{\sigma}_{i}^{S}\right)^{2}, \\
M^{N}\left(\mathbf{a}^{e}, \mathbf{a}_{t-L_{\max }^{u}}^{u}, \ldots, \mathbf{a}_{t}^{u}\right) & =\sum_{i=1}^{n}\left(a_{i}^{e} \mu_{i}^{N}+\sum_{T=0}^{L_{\max }^{i}} a_{i, t-T}^{u} p_{i, T}^{L}\right), \\
\left(\Sigma^{N}\left(\mathbf{a}^{e}, \mathbf{a}_{t-L_{\max }^{u}}^{u}, \ldots, \mathbf{a}_{t}^{u}\right)\right)^{2} & =\sum_{i=1}^{n}\left(\sum_{k=1}^{K_{i}} q_{i k}^{2}\right)\left(\sum_{T=0}^{L_{\max }^{i}}\left(a_{i}^{e}+a_{i, t-T}^{u}\right)^{2} p_{i, T}^{L}\left(1-p_{i, T}^{L}\right)\right) .
\end{aligned}
$$

In addition, suppose that for each procedure type $i=1, \ldots, n$ there exists $\delta_{i}>0$ such that

$$
\lim _{K_{i} \rightarrow \infty} \frac{\left(\sum_{k=1}^{K_{i}} q_{i k}^{2+\delta_{i}}\right)^{\frac{1}{2+\delta_{i}}}}{\left(\sum_{k=1}^{K_{i}} q_{i k}^{2}\right)^{\frac{1}{2}}}=0 .
$$

Then, as $K_{i} \rightarrow \infty$,

$$
\frac{D_{t}\left(\mathbf{a}^{e}, \mathbf{a}_{t}^{u}\right)-M^{S}\left(\mathbf{a}^{e}, \mathbf{a}_{t}^{u}\right)}{\Sigma^{S}\left(\mathbf{a}^{e}, \mathbf{a}_{t}^{u}\right)} \stackrel{d}{\rightarrow} \mathcal{N}(0,1)
$$

and

$$
\frac{O_{t}\left(\mathbf{a}^{e}, \mathbf{a}_{t-L_{\max }^{u}}^{u}, \ldots, \mathbf{a}_{t}^{u}\right)-M^{N}\left(\mathbf{a}^{e}, \mathbf{a}_{t-L_{\max }^{u}}^{u}, \ldots, \mathbf{a}_{t}^{u}\right)}{\Sigma^{N}\left(\mathbf{a}^{e}, \mathbf{a}_{t-L_{\max }^{u}}^{u}, \ldots, \mathbf{a}_{t}^{u}\right)} \stackrel{d}{\rightarrow} \mathcal{N}(0,1) .
$$

Proposition 1 derives normal approximations for the total duration of all surgical procedures, $D_{t}\left(\mathbf{a}^{e}, \mathbf{a}_{t}^{u}\right)$, for given numbers of elective and urgent procedures on day $t$, as well as for the bed occupancy, $O_{t}\left(\mathbf{a}^{e}, \mathbf{a}_{t-L_{\max }}^{u}, \ldots, \mathbf{a}_{t}^{u}\right)$, for given numbers of elective and urgent procedures performed in the last $L_{\max }$ days. These approximations involve easy-to-calculate parameters $M^{S}\left(\mathbf{a}^{e}, \mathbf{a}_{t}^{u}\right)$, $\Sigma^{S}\left(\mathbf{a}^{e}, \mathbf{a}_{t}^{u}\right), M^{N}\left(\mathbf{a}^{e}, \mathbf{a}_{t-L_{\max }^{u}}^{u}, \ldots, \mathbf{a}_{t}^{u}\right)$ and $\Sigma^{N}\left(\mathbf{a}^{e}, \mathbf{a}_{t-L_{\max }}^{u}, \ldots, \mathbf{a}_{t}^{u}\right)$ that are based only on the first two moments of the distributions of surgical durations and patient length-of-stay. Thus, Proposition 1 provides a powerful tool for studying trade-offs faced by a hospital designing the optimal portfolio of elective procedures under arbitrary procedure and length-of-stay durations. The power of this approximation, however, comes at a cost, as (25) and (26) rely on two assumptions. First, these normal approximations become exact representations of the underlying distributions only in the asymptotic regime of $K_{i} \rightarrow \infty$. Second, they require the condition (24) that describes a setting where, as the number of surgical teams performing the same procedure grows, none of those teams "dominates" others in terms of the share of procedures it performs. Given these assumptions, it 
is important to establish how the approximations outlined in Proposition 1 perform in realistic settings that allow for low values of $K_{i}$ and "hoarding" of procedures by one or more surgical teams. With this in mind, we provide an analytical comparison between the exact and approximated optimal portfolios of surgical procedures in a special case in Section B.2 of the Appendix, as well as numerical investigations of the quality of the approximations in Section 6.2.

The often-observed pronounced correlation among the procedures performed by the same surgical team imposes a significant analytical challenge for a tractable analysis of the impact of selected set of elective procedures on the use of hospital resources, and condition (24) is introduced to deal with this challenge. To underscore the impact of this correlation, we derive a second CLT-based approximation, presented in Proposition A1 of the Appendix, that calculates the expressions for the use of OR time and recovery beds under the alternative assumption of the independence of the instances of surgical duration and LOS pairs produced by the same surgical team. Contrasting the two results underscores two important points. First, both results are asymptotic, with Proposition 1 holding in the limit of $K_{i} \rightarrow \infty$, and Proposition A1 in the limit of $a_{i} \rightarrow \infty$. Second, under the two alternative correlational structures, the expressions for the variance of the OR time and bed usage are qualitatively different, although their first moments are identical. In particular, the perfectly correlated durations produce, for the same numbers of daily procedures, higher variance in terms of the use of both resources. This, in turn, results in higher "overage" costs, and, consequently, may produce, for the same values of penalty $\operatorname{costs} \pi^{N}$ and $\pi^{S}$, smaller optimal numbers of daily elective procedures. Thus, ignoring the correlation structure described in the Assumption 5 may result in overutilization of hospital resources. With this in mind, we conduct our analysis below based on the more realistic Assumption 5 and the limiting distributions derived in Proposition 1.

Proposition 1 enables the analytical characterization of the optimal portfolio of surgical procedures, as it leads to a closed-form expression for the limiting value of the objective function (19), as given by the following lemma.

Lemma 1. Under the limiting distributions for $O_{t}(\cdot)$ and $D_{t}(\cdot)$ given in Proposition 1 , the expected daily profit (19) can be approximated as

$$
\begin{aligned}
& \Pi\left(\mathbf{a}^{e}\right)=\sum_{i=1}^{n} R_{i}\left(a_{i}^{e}+\bar{\xi}_{i}\right)-\pi^{S} \int_{l_{1}}^{h_{1}} \ldots \int_{l_{n}}^{h_{n}} d \xi_{1, t} \ldots d \xi_{n, t} f\left(\xi_{1, t}, \ldots, \xi_{n, t}\right) \Sigma^{S}\left(\mathbf{a}^{e}, \boldsymbol{\xi}_{t}\right) \times \\
& \left(\frac{1}{\sqrt{2 \pi}} e^{-\frac{1}{2}\left(\frac{\tau-M^{S}\left(\mathbf{a}^{e}, \boldsymbol{\xi}_{t}\right)}{\Sigma^{S}\left(\mathbf{a}^{e}, \boldsymbol{\xi}_{t}\right)}\right)^{2}}-\left(\frac{\tau-M^{S}\left(\mathbf{a}^{e}, \boldsymbol{\xi}_{t}\right)}{2 \Sigma^{S}\left(\mathbf{a}^{e}, \boldsymbol{\xi}_{t}\right)}\right) \operatorname{erfc}\left(\frac{\tau-M^{S}\left(\mathbf{a}^{e}, \boldsymbol{\xi}_{t}\right)}{\sqrt{2} \Sigma^{S}\left(\mathbf{a}^{e}, \boldsymbol{\xi}_{t}\right)}\right)\right) \\
& -\pi^{N} \int_{l_{1}}^{h_{1}} \ldots \int_{l_{n}}^{h_{n}} d \boldsymbol{\xi}_{t-L_{\max }} f^{u}\left(\boldsymbol{\xi}_{t-L_{\max }}\right) \ldots \int_{l_{1}}^{h_{1}} \ldots \int_{l_{n}}^{h_{n}} d \boldsymbol{\xi}_{t} f^{u}\left(\boldsymbol{\xi}_{t}\right)
\end{aligned}
$$




$$
\begin{aligned}
& \times \Sigma^{N}\left(\mathbf{a}^{e}, \boldsymbol{\xi}_{t-L_{\max }}, \ldots, \boldsymbol{\xi}_{t}\right) \times\left(\frac{1}{\sqrt{2 \pi}} e^{-\frac{1}{2}\left(\frac{c-M^{N}\left(\mathbf{a}^{e}, \boldsymbol{\xi}_{t-L \max }, \ldots, \boldsymbol{\xi}_{t}\right)}{\Sigma^{N}\left(\mathbf{a}^{e}, \boldsymbol{\xi}_{t-L \max }, \ldots, \boldsymbol{\xi}_{t}\right)}\right)^{2}}\right. \\
& \left.-\left(\frac{c-M^{N}\left(\mathbf{a}^{e}, \boldsymbol{\xi}_{t-L_{\max }}, \ldots, \boldsymbol{\xi}_{t}\right)}{2 \Sigma^{N}\left(\mathbf{a}^{e}, \boldsymbol{\xi}_{t-L_{\max }}, \ldots, \boldsymbol{\xi}_{t}\right)}\right) \operatorname{erfc}\left(\frac{c-M^{N}\left(\mathbf{a}^{e}, \boldsymbol{\xi}_{t-L_{\max }}, \ldots, \boldsymbol{\xi}_{t}\right)}{\sqrt{2} \Sigma^{N}\left(\mathbf{a}^{e}, \boldsymbol{\xi}_{t-L_{\max }}, \ldots, \boldsymbol{\xi}_{t}\right)}\right)\right),
\end{aligned}
$$

where

$$
\operatorname{erfc}(x)=\frac{2}{\sqrt{\pi}} \int_{x}^{+\infty} e^{-t^{2}} d t
$$

is the complementary error function.

Then, the problem of finding the optimal portfolio of surgical procedures can be formulated as

$$
\max _{0 \leq \mathbf{a}^{e} \leq \mathbf{U}} \Pi\left(\mathbf{a}^{e}\right)
$$

where $\mathbf{U}=\left(U_{1}, U_{2}, \ldots, U_{n}\right)$.

The optimization problem (29) reflects a setting where multiple types of surgical procedures compete for hospital resources. Note that in this optimization problem we assume that the hospital is a profit maximizing entity. In practice, hospitals can have altruistic and other long-run considerations, such as preventing excessive wait times on certain procedures. The upper bounds on the number of surgical procedures can be used to represent such considerations as well. Extending the problem by including lower bounds is also possible.

Among the hospital resources, OR capacity is often considered to be most important, as it is tied to the main source of hospital revenue. Ignoring the costs that surgical procedures may impose on other hospital resources, such as recovery bed capacity, reduces (29) to a single-resource case that may be viewed as a variant of the inverse newsvendor problem analyzed in Carr and Lovejoy (2000), where the optimal matching between the demand and supply of a single resource is achieved via controlling the demand side. To underscore the novelty of our modeling and analysis, we emphasize the importance of accounting for the impact of a given portfolio of surgical procedures on multiple resources, and, from that perspective, our model may be described as a "multidimensional inverse newsvendor." Below we start with the case of a single procedure type and then proceed to extend our analysis to multiple procedure types.

\section{Managing Hospital Service Capacity in a Single-Specialty Hospital}

For a small number of specialty (e.g., cardiac or orthopedic) hospitals or single-specialty hospitals (SSH), a particular class of surgical procedures may dominate the portfolio mix. In such a setting, the hospital is faced with the problem of balancing the revenues it receives with the potentially unequal impact that procedures have on OR and recovery bed capacities. In addition, while many 
SSHs specialize in elective procedures, about $42 \%$ of them have an emergency department, so that some of the performed procedures may be nonelective (U.S. Government Accountability Office 2003).

In this section, we analyze the problem of optimizing the number of elective procedures for a single-specialty hospital in the presence of urgent procedures. In particular, we first analyze the case of arbitrary distributions of surgery and LOS durations using the CLT-based approximations for the total required OR and recovery bed capacities derived in Proposition 1.

When considering a setting where a hospital specializes in a particular surgical procedure, we let $l$ and $h$ be the lower and the upper bound on the daily number of urgent surgical procedures, respectively, and let $f(\xi)$ be the corresponding pdf. In the case of a single procedure type, we drop the procedure index and use the following notation: $a^{e}=a_{1}^{e}, R=R_{1}, \mu^{S}=\mu_{1}^{S}, \sigma^{S}=\sigma_{1}^{S}, \bar{\sigma}^{S}=\bar{\sigma}_{1}^{S}$, $\mu^{N}=\mu_{1}^{N}, \sigma^{N}=\sigma_{1}^{N}, \bar{\sigma}^{N}=\bar{\sigma}_{1}^{N}, K=K_{1}, q_{k}=q_{1 k}, k \in\{1, \ldots, K\}, l=l^{1}, h=h^{1}, \bar{\xi}=\bar{\xi}_{1}, U=U_{1}$. We also define

$$
\begin{aligned}
\rho^{N}\left(a^{e}, a_{t-L_{\max }^{u}}^{u}, \ldots, a_{t}^{u}\right) & =\frac{M^{N}\left(a^{e}, a_{t-L_{\max }}^{u}, \ldots, a_{t}^{u}\right)}{\Sigma^{N}\left(a^{e}, a_{t-L_{\max }^{u}}^{u}, \ldots, a_{t}^{u}\right)}, \\
\rho^{S} & =\frac{\mu^{S}}{\bar{\sigma}^{S}} .
\end{aligned}
$$

For the analysis below it is convenient to use the following function:

$$
\begin{array}{r}
H(x)=\frac{\pi^{S} \bar{\sigma}^{S}}{\sqrt{2 \pi}}\left(\int_{l}^{h} d \xi_{t} f^{u}\left(\xi_{t}\right)\left(e^{-\frac{1}{2}\left(\frac{\tau}{\left(x+\xi_{t}\right) \bar{\sigma}^{S}}-\rho^{S}\right)^{2}}+\rho^{S} \int_{\frac{\tau}{\left(x+\xi_{t}\right) \bar{\sigma}^{S}}-\rho^{S}}^{+\infty} e^{-\frac{1}{2} v^{2}} d v\right)\right) \\
+\frac{\pi^{N}}{\sqrt{2 \pi}}\left(\int _ { \Xi ^ { u } } d \boldsymbol { \Xi } _ { t } F ^ { u } ( \boldsymbol { \Xi } _ { t } ) \left(\frac{\sqrt{\sum_{k=1}^{K} q_{k}^{2}}}{\Sigma^{N}\left(x, \Xi_{t}\right)}\left(\sum_{T=0}^{L_{\max }}\left(x+\xi_{t-T}\right) p_{T}^{L}\left(1-p_{T}^{L}\right)\right) e^{-\frac{1}{2}\left(\frac{c-M^{N}\left(x, \Xi_{t}\right)}{\Sigma^{N}\left(x, \mathbf{\Xi}_{t}\right)}\right)^{2}}\right.\right. \\
\left.\left.+\mu^{N} \int_{\frac{c-M^{N}\left(x, \mathbf{\Xi}_{t}\right)}{\Sigma^{N}\left(x, \mathbf{\Xi}_{t}\right)}}^{+\infty} e^{-\frac{1}{2} v^{2}} d v\right)\right),
\end{array}
$$

where we set

$$
\int_{\Xi^{u}} d \boldsymbol{\Xi}_{t} F^{u}\left(\boldsymbol{\Xi}_{t}\right)=\int_{l}^{h} \cdots \int_{l}^{h} d \xi_{t-L_{\max }} \cdots d \xi_{t} f^{u}\left(\xi_{t-L_{\max }}\right) \cdots f^{u}\left(\xi_{t}\right)
$$

with

$$
\begin{aligned}
& \boldsymbol{\Xi}^{u}=\underbrace{[l, h] \otimes \cdots \otimes[l, h]}_{L_{\max }+1 \text { times }}=[l, h]^{\otimes\left(L_{\max }+1\right)}, \\
& \boldsymbol{\Xi}_{t}=\left(\xi_{t-L_{\max }} \cdots, \xi_{t}\right),
\end{aligned}
$$




$$
\begin{aligned}
d \boldsymbol{\Xi}_{t} & =d \xi_{t-L_{\max }} \cdots d \xi_{t}, \\
F^{u}\left(\boldsymbol{\Xi}_{t}\right) & =\prod_{T=0}^{L_{\max }} f^{u}\left(\xi_{t-T}\right) .
\end{aligned}
$$

Note that $\partial \Pi / \partial a^{e}=R-H\left(a^{e}\right)$.

We assume that both the OR and bed capacities are sufficient to accommodate, in expectation, the maximum number of urgent procedures. In other words, $h$ is bounded from above by $\hat{h}$, where $\hat{h}=\min \left\{\frac{\tau}{\mu^{S}}, \frac{c}{\mu^{N}}\right\}:$

$$
h<\hat{h} \text {. }
$$

The following result describes the optimal number of elective surgical procedures.

Proposition 2. In a setting with a single procedure type, the following statements hold.

a) The expected daily profit function $\Pi\left(a^{e}\right)$ is a concave function of $a^{e}$.

b) For

$$
R \leq H(0)
$$

the optimal daily number of procedures $\hat{a}^{e}$ is 0 .

c) For

$$
\begin{aligned}
R>H(+\infty)= & \frac{\pi^{N} \bar{\sigma}^{N}}{\sqrt{2 \pi}}\left(e^{-\frac{\mu_{N}^{2}}{2\left(\bar{\sigma}^{N}\right)^{2}}}+\sqrt{\frac{\pi}{2}} \frac{\mu_{N}}{\overline{\sigma^{N}}} \operatorname{erfc}\left(\frac{1}{\sqrt{2}}\left(-\frac{\mu_{N}}{\bar{\sigma}^{N}}\right)\right)\right) \\
& +\frac{\pi^{S} \bar{\sigma}^{S}}{\sqrt{2 \pi}}\left(e^{-\frac{1}{2}\left(\rho^{S}\right)^{2}}+\sqrt{\frac{\pi}{2}} \rho^{S} \operatorname{erfc}\left(\frac{1}{\sqrt{2}}\left(-\rho^{S}\right)\right)\right),
\end{aligned}
$$

the optimal daily number of procedures $\hat{a}^{e}$ is equal to the upper bound $U$.

d) For

$$
H(0)<R<H(+\infty)
$$

the optimal daily number of procedures is given by:

$$
\hat{a}^{e}=\min \left\{H^{-1}(R), U\right\} .
$$

e) The optimal daily number of procedures, $\hat{a}^{e}$, is an increasing function of $R, c$, and $\tau$, and a decreasing function of $\pi^{N}, \pi^{S}, \mu^{S}$, and $\mu^{N}$. Moreover, when

$$
R<H(\hat{h}-h)
$$


$\hat{a}^{e}$ is decreasing in $\bar{\sigma}^{S}$.

f) Consider two cdf functions for the daily number of urgent procedures, $\Phi^{1}(\xi)$ and $\Phi^{2}(\xi)$ such that the first distribution exhibits first-order stochastic dominance over the second,

$$
\Phi^{1}(\xi) \leq \Phi^{2}(\xi), \forall \xi \in[l, h]
$$

and let $\hat{a}^{e, 1}$ and $\hat{a}^{e, 2}$ be the optimal daily numbers of elective surgical procedures corresponding to $\Phi^{1}(\xi)$ and $\Phi^{2}(\xi)$, respectively. Then, $\hat{a}^{e, 1} \leq \hat{a}^{e, 2}$.

Proposition 2 expresses the optimal number of surgical procedures $\hat{a}^{e}$, when it is strictly positive, as a unique root of the one-dimensional transcendental equation $H\left(\hat{a}^{e}\right)=R$ that can be solved numerically.

Figure 1 illustrates the dependence of the optimal daily number of elective procedures on the parameters of the distribution of the daily number of urgent procedures in the setting where the number of urgent procedures can take two values: $l=0$ with probability $1-q_{h}$, and $h$ with probability $q_{h}$. In this Figure, to illustrate the impact of the demand limitations we are using a setting where the maximum daily number of elective procedures is set at $45 \%$ of the value that matches, in expectation, the capacity of the bottleneck resource. Also, note that the curves in Figure 1 are based on the CLT-based approximation for the hospital profit function described in Lemma 1, and, therefore, do not depend on the $K_{i}$ values.

As part (f) of Proposition 2 implies, a hospital's ability to accommodate elective procedures declines as the number of urgent surgeries stochastically increases. In particular, higher values of $h$ and $q_{h}$ both force diminished optimal values of $\hat{a}^{e}$, with elective procedures being "squeezed out" completely once these parameters reach critical levels.

Figure 2 illustrates the results of Proposition 2 for four settings with different levels of penalty $\operatorname{costs} \pi^{N}$ and $\pi^{S}$ and the same distribution of the daily number of urgent procedures as used in Figure 1 with $q_{h}=0.5$ and $h=10$. Note that for all settings, the optimal number of procedures approaches the following limit when there is ample bed capacity:

$$
\hat{a}_{S}^{e}=\underset{a^{e} \geq 0}{\arg \max }\left(\Pi\left(a^{e}\right) \mid \pi^{N}=0\right) .
$$

This limiting value is a decreasing function of $\pi^{S}$ and is an important benchmark as it represents the optimal number of procedures under the "front-end" planning approach that focuses exclusively on optimal utilization of operating theater resources, ignoring the potential impact on "backroom" capacity, such as recovery resources. The optimal procedure numbers in Figure 2 display the monotonicity properties described in Proposition 2. Note that, unlike the other three cases, the setting with the lowest values of penalty costs, $\pi^{N}=0.5$ and $\pi^{S}=1.5$, exhibits a positive 


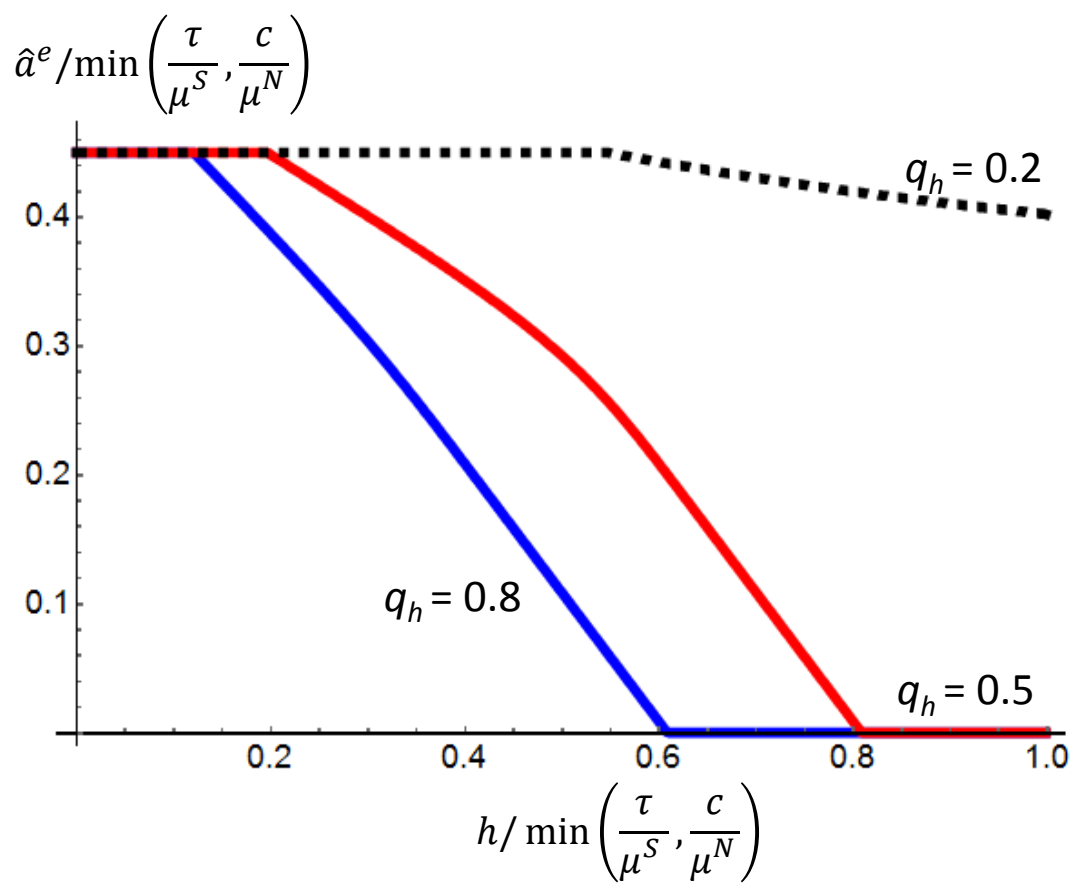

Figure 1 : Optimal expected surgical load $\hat{a}^{e}$ for the two-value distribution of the daily number of urgent procedures for different values of the "high" value, $h$, and its probability, $q_{h}\left(U=0.45 \min \left(\frac{\tau}{\mu^{S}}, \frac{c}{\mu^{N}}\right), R=1, \tau=\right.$ $\left.50, c=100, \mu^{N}=\bar{\sigma}^{N}=\mu^{S}=\bar{\sigma}^{S}=1, \pi^{N}=\pi^{S}=2, l=0\right)$.

limit $\hat{a}_{S}^{e}=\lim _{c \rightarrow 0} \hat{a}^{e}$. In this setting, even severe restrictions on the recovery bed capacity will not prevent the hospital from putting a significant load on its surgical resources. Such an approach is optimal in settings where the penalty cost associated with exceeding the recovery bed capacity is low.

Figure 3 shows how the ratio of the profit values obtained for $a_{e}=\hat{a}_{S}^{e}$ and $a_{e}=\hat{a}^{e}$ depends on the recovery bed capacity in four settings illustrated in Figure 2. When bed capacity is too tight or the process of alleviating bed shortages is too expensive, the performance deterioration associated with ignoring the "backroom" processes can become acute, leading to financial losses. At the same time, the "front-end" approach to determining the number of surgical procedures can be effective in settings with ample bed capacity or a low penalty associated with bed capacity overload. Note that since $\hat{a}_{S}^{e}$ is the optimal number of daily elective procedures for $\pi^{N}=0$, the monotonicity results of Proposition 2 imply that $\hat{a}_{S}^{e} \geq \hat{a}^{e}$.

We can interpret the ratio $R / \pi^{S}$ as the maximum amount of excess operating room time that the reward, $R$, can "cover." Our next result derives an upper bound, $\epsilon^{S}$ :

$$
\epsilon^{S}=\left(\frac{\pi^{N}}{\pi^{S} \bar{\sigma}^{S}}\right) \frac{I_{2}^{N}-\left(c-\left(\frac{\tau}{\mu^{S}}-h\right) \mu^{N}\right) I_{0}^{N}+I_{1}^{N}}{\sqrt{2 \pi}\left(\bar{\xi}+\frac{\tau}{\mu^{S}}\right)\left(\frac{R}{\pi^{S} \bar{\sigma}^{S}}\right)-\frac{\tau}{\mu^{S}} J_{1}^{S}-J_{2}^{S}-\rho^{S} I_{2}^{S}},
$$




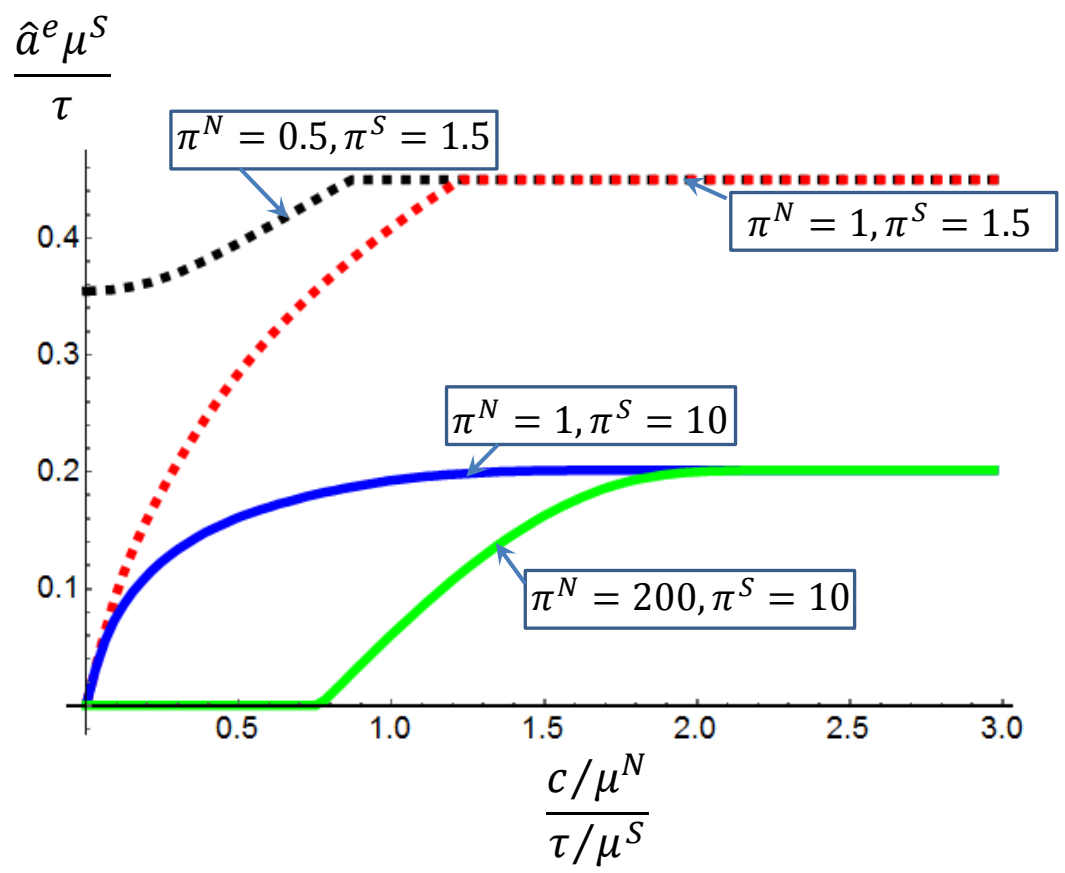

Figure 2: Optimal expected daily surgical load from elective procedures, $\frac{\hat{a}^{e} \mu^{S}}{\tau}$, as a function of the bed capacity factor $\frac{\frac{c}{\mu^{N}}}{\frac{\tau}{\mu^{S}}}$ for different values of penalty $\operatorname{costs} \pi^{N}$ and $\pi^{S}\left(U=0.45 \min \left(\frac{\tau}{\mu^{S}}, \frac{c}{\mu^{N}}\right), R=1, \tau=50, \mu^{N}=\right.$ $\left.1, \bar{\sigma}^{N}=1, \mu^{S}=1, \bar{\sigma}^{S}=1, q_{h}=0.5, l=0, h=10\right)$.

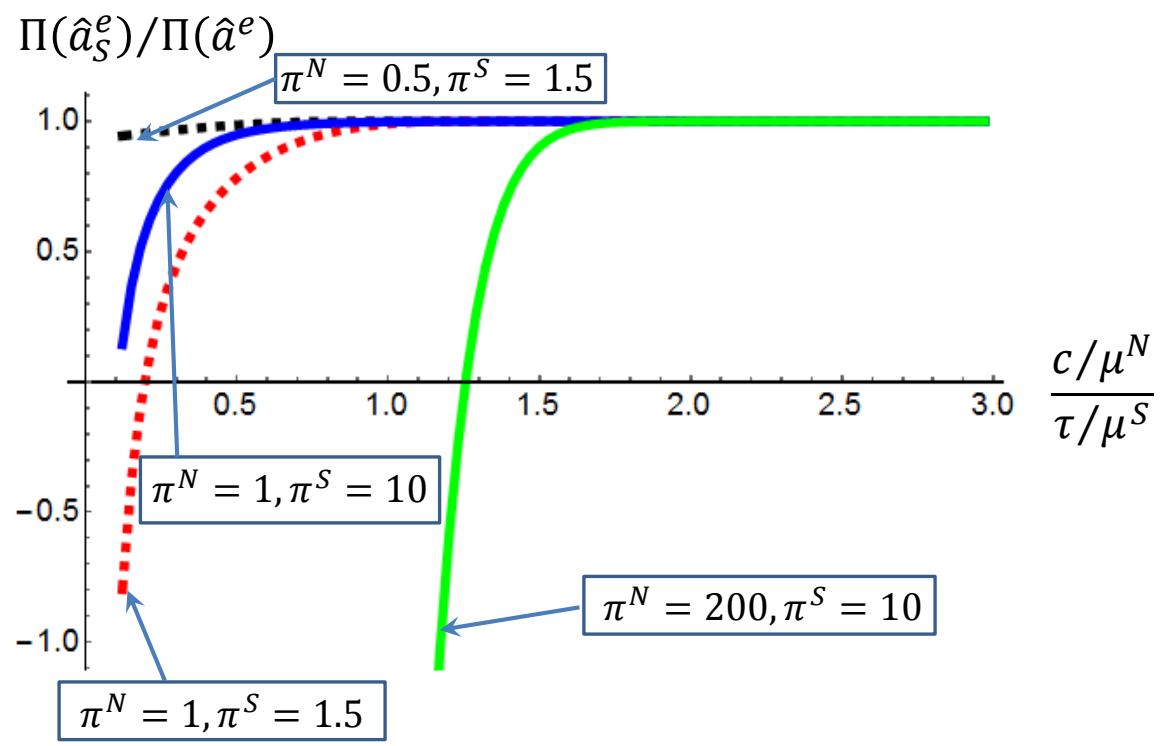

Figure 3 : Relative performance of the "front-end" approach to selecting the number of elective surgical procedures, $\frac{\Pi\left(\hat{a}_{S}^{e}\right)}{\Pi\left(\hat{a}^{e}\right)}$, as a function of the bed capacity factor $\frac{\frac{c}{\mu^{N}}}{\frac{\tau^{S}}{\mu^{S}}}$ for different values of penalty $\operatorname{costs} \pi^{N}$ and $\pi^{S}$ $\left(U=0.45 \min \left(\frac{\tau}{\mu^{S}}, \frac{c}{\mu^{N}}\right), R=1, \tau=50, \mu^{N}=1, \bar{\sigma}^{N}=1, \mu^{S}=1, \bar{\sigma}^{S}=1, q_{h}=0.5, l=0, h=10\right)$. 
on the relative performance of the "front-end" approach when the ratio $R / \pi^{S}$ is bounded by $B_{L}$ and $B_{H}$ :

$$
\begin{aligned}
B_{L} & =\frac{\bar{\sigma}^{S}}{\sqrt{2 \pi}}\left(\frac{1}{\bar{\xi}+\frac{\tau}{\mu^{S}}}\right)\left(\frac{\tau}{\mu^{S}} I_{1}^{S}+J_{2}^{S}+\rho^{S} I_{2}^{S}\right), \\
B_{H} & =\frac{\bar{\sigma}^{S}}{\sqrt{2 \pi}}\left(J_{1}^{S}+\rho^{S} I_{1}^{S}\right),
\end{aligned}
$$

where

$$
\begin{aligned}
& I_{0}^{N}=\int_{\boldsymbol{\Xi}^{u}} d \boldsymbol{\Xi}_{t} F^{u}\left(\boldsymbol{\Xi}_{t}\right) \quad \int^{+\infty} e^{-\frac{1}{2} v^{2}} d v, \\
& \frac{c-M^{N}\left(\frac{\tau}{\mu^{S}}-h, \mathbf{\Xi}_{t}\right)}{\Sigma^{N}\left(\frac{\tau}{\mu^{S}}-h, \Xi_{t}\right)} \\
& I_{1}^{N}=\int_{\Xi^{u}} d \boldsymbol{\Xi}_{t} F^{u}\left(\boldsymbol{\Xi}_{t}\right) \sum_{T=0}^{L_{\max }} p_{T}^{L} \xi_{t-T} \int^{+\infty} e^{-\frac{1}{2} v^{2}} d v, \\
& \frac{c-M^{N}\left(\frac{\tau}{\mu^{S}}-h, \mathbf{\Xi}_{t}\right)}{\Sigma^{N}\left(\frac{\tau}{\mu^{S}}-h, \mathbf{\Xi}_{t}\right)} \\
& I_{2}^{N}=\int_{\boldsymbol{\Xi}^{u}} d \boldsymbol{\Xi}_{t} F^{u}\left(\boldsymbol{\Xi}_{t}\right) \Sigma^{N}\left(\frac{\tau}{\mu^{S}}-h, \boldsymbol{\Xi}_{t}\right) e^{-\frac{1}{2}\left(\frac{c-M^{N}\left(\frac{\tau}{\mu^{S}}-h, \boldsymbol{\Xi}_{t}\right)}{\Sigma^{N}\left(\frac{\tau}{\mu^{S}}-h, \boldsymbol{\Xi}_{t}\right)}\right)^{2}}, \\
& I_{1}^{S}=\sqrt{\frac{\pi}{2}} \int_{l}^{h} d \xi f(\xi) \operatorname{erfc}\left(\frac{1}{\sqrt{2}}\left(\frac{\tau}{\left(\frac{\tau}{\mu^{S}}-h+\xi\right) \bar{\sigma}^{S}}-\rho^{S}\right)\right), \\
& I_{2}^{S}=\sqrt{\frac{\pi}{2}} \int_{l}^{h} d \xi f(\xi) \xi \operatorname{erfc}\left(\frac{1}{\sqrt{2}}\left(\frac{\tau}{\left(\frac{\tau}{\mu^{S}}-h+\xi\right) \bar{\sigma}^{S}}-\rho^{S}\right)\right), \\
& J_{1}^{S}=\int_{l}^{h} d \xi f(\xi) e^{-\frac{1}{2}\left(\frac{\tau}{\left(\frac{\tau}{\mu^{S}}-h+\xi\right) \bar{\sigma}^{S}}-\rho^{S}\right)^{2}} \\
& J_{2}^{S}=\int_{l}^{h} d \xi f(\xi) \xi e^{-\frac{1}{2}\left(\frac{\tau}{\left(\frac{\tau}{\mu^{S}}-h+\xi\right) \bar{\sigma}^{S}}-\rho^{S}\right)^{2}} .
\end{aligned}
$$

Then, the relative performance of the "front-end" approach can be described as follows.

Proposition 3. a) For

$$
B_{L}<\frac{R}{\pi^{S}}<B_{H}
$$

we have

$$
\frac{\Pi\left(\hat{a}^{e}\right)-\Pi\left(\hat{a}_{S}^{e}\right)}{\Pi\left(\hat{a}^{e}\right)} \leq \epsilon^{S} .
$$


b) Consider two cdf functions for the daily number of urgent procedures, $\Phi^{1}(\xi)$ and $\Phi^{2}(\xi)$, both strictly increasing on $\xi \in[l, h]$, such that

$$
\begin{aligned}
\Phi^{i}(l) & =0, i=1,2, \\
\Phi^{i}(h) & =1, i=1,2, \\
\Phi^{1}(\xi) & \leq \Phi^{2}(\xi), \forall \xi \in[l, h],
\end{aligned}
$$

and let, under (56), $\epsilon^{S, 1}$ and $\epsilon^{S, 2}$ be the upper bound values defined by (57) for $\Phi^{1}(\xi)$ and $\Phi^{2}(\xi)$, respectively. Then, $\epsilon^{S, 2} \leq \epsilon^{S, 1}$.

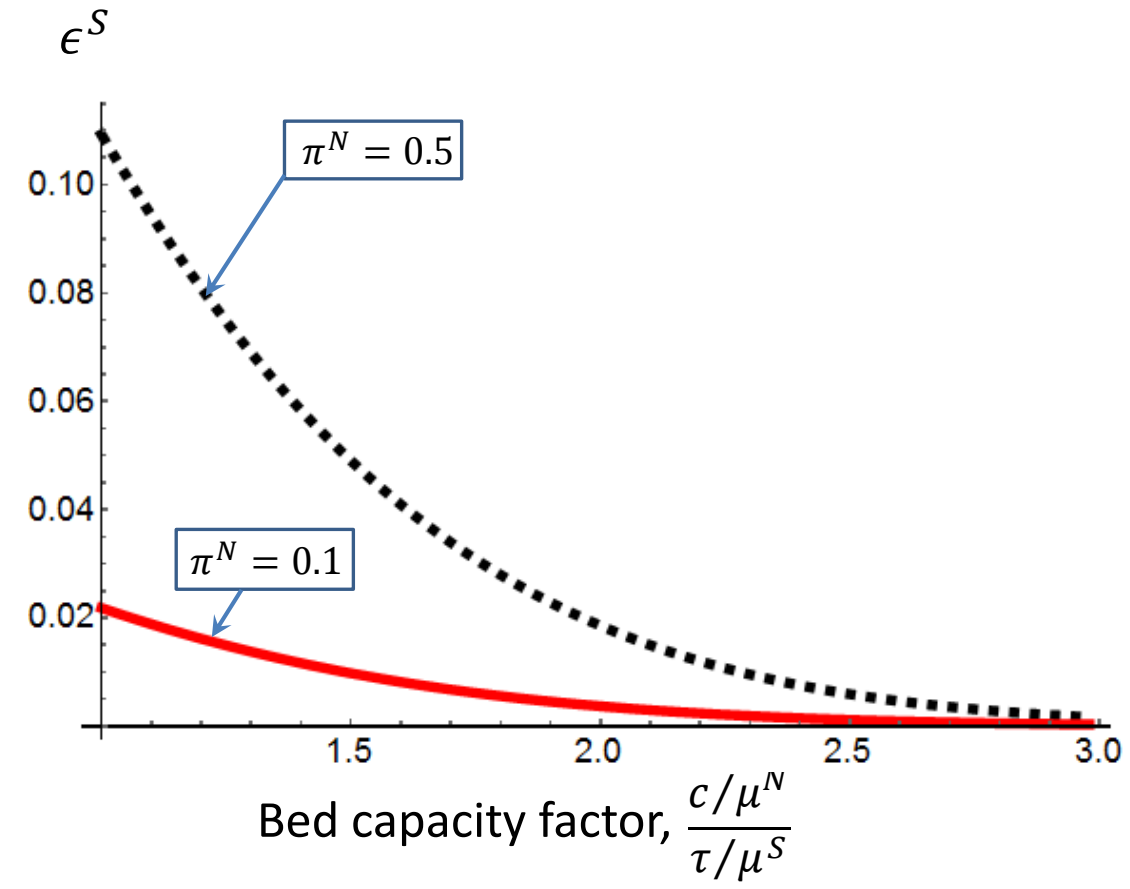

Figure 4: The upper bound $\epsilon^{S}$ on the relative profit loss generated by the "front-end" approach as a function of the bed capacity factor $\frac{\frac{c}{\mu^{N}}}{\frac{\tau}{\mu^{S}}}$ for different values of penalty cost $\pi^{N}\left(R=3.5, \tau=50, \mu^{N}=1, \bar{\sigma}^{N}=1, \mu^{S}=\right.$ $\left.1, \bar{\sigma}^{S}=1, \pi^{S}=5, q_{h}=0.5, l=0, h=10\right)$.

The upper bound on the relative profit loss resulting from ignoring the "backroom" capacity, as described by Proposition 3, is a decreasing function of the bed capacity $c$ and is proportional to the bed overload $\operatorname{cost} \pi^{N}$. Figure 4 shows the exponential decline of the performance gap $\epsilon^{S}$ with the increasing recovery bed capacity $c$ for two different values of the penalty $\operatorname{cost} \pi^{N}$.

Figure 5 illustrates part (b) of Proposition 3 in the setting with the two-value distribution of the daily number of urgent procedures with $l=0$ and $h=10$. In particular, Figure 5 shows how the upper bound $\epsilon^{S}$ changes as a function of the probability of the "high" value $q_{h}$. 


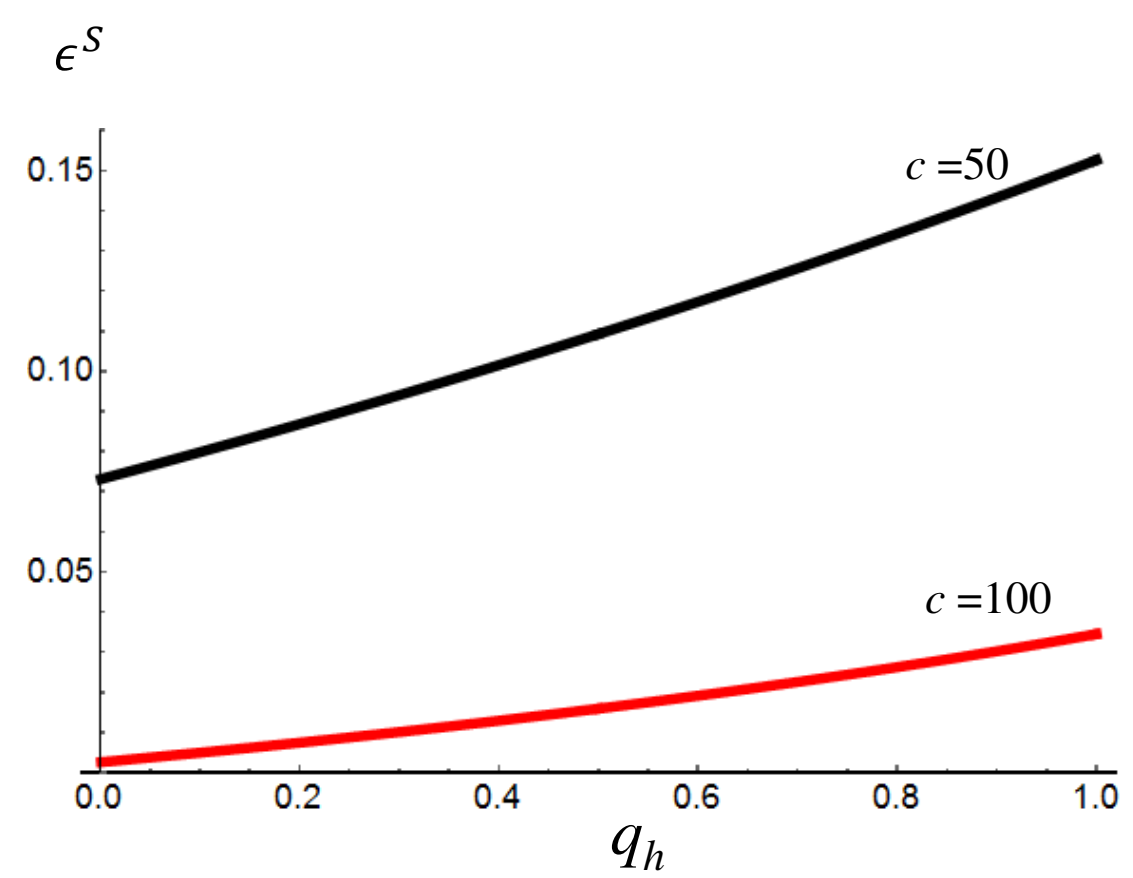

Figure 5: The upper bound $\epsilon^{S}$ on the relative profit loss generated by the "front-end" approach for the two-value distribution of the daily number of urgent procedures as the function of the probability $q_{h}$ of the "high" value for two values of bed capacity $c\left(R=3.5, \tau=50, \mu^{N}=\bar{\sigma}^{N}=\mu^{S}=\bar{\sigma}^{S}=1, \pi^{N}=0.5, \pi^{S}=5\right.$, $l=0, h=10)$.

To investigate the quality of the approximation suggested by Proposition 1, in Section B.2 of the Appendix we develop closed-form expressions of the optimal number of elective procedures for a special case where both the surgical times and patient LOS durations have two-point distributions. The theoretical and numerical analyses of this case indicate that the CLT-based approach combines a clear benefit of being able to handle arbitrary surgery and LOS distributions with an adequate performance in settings where a proper accounting must be made for the presence of a limited "backroom" capacity.

\section{Managing Hospital Service Capacity: Two Procedure Types}

The majority of hospitals in the U.S. perform multiple types of surgical procedures. Below we extend our analysis of Section 4 to this more common setting. When multiple procedure types utilize multiple hospital resources, the trade-off associated with balancing the revenues of a particular procedure with the "uneven" cost pressure it exerts on different resources is further complicated by the competition for each resource among different types of procedures. To isolate the effects of this trade-off, we assume that there are no urgent procedures. Our analysis of the multiple-type setting focuses on the case of two procedure types that provides important insights on the optimal resolution of such trade-off, whereas the analysis of the case with $n>2$ procedure types in the 
absence of upper bounds on the numbers of elective procedures is included in Section B.1 of the Appendix.

For the case of two procedure types, we look at special settings in order to obtain an analytical characterization of the optimal portfolio of elective procedures. In the presence of two constraining resources, such as recovery bed capacity and OR time, an important special case to analyze is the one where different surgical procedures exhibit different amounts of "pressure" on each resource. In particular, we consider two settings we call "asymmetric" and "proportional asymmetric" where there is a strong asymmetry in the variance values for the required surgery time and the recovery bed capacity. Formally, we use the following definition.

Definition 1. The setting with two procedure types is asymmetric if

$$
\sigma_{1}^{S}=\sigma_{2}^{N}=0
$$

The setting with two procedure types is proportional asymmetric if, in addition to (61), we have

$$
\begin{aligned}
& r_{S}=\frac{R_{1}}{\mu_{1}^{S}}=\frac{R_{2}}{\mu_{2}^{S}}, \\
& r_{N}=\frac{R_{1}}{\mu_{1}^{N}}=\frac{R_{2}}{\mu_{2}^{N}} .
\end{aligned}
$$

As Definition 1 implies, in the asymmetric setting procedures of type 1 result in completely predictable surgery durations, while procedures of type 2 in completely predictable LOS durations. The proportional asymmetric setting combines the acute asymmetry in the stochastic components of procedure impact on two resources with complete symmetry in terms of the expected compensation for the use of these resources, on a per-unit-of-time basis. As we will show below, the combination of these features will allow us to put a sharper focus on the mechanism of the optimal portfolio construction.

Similar to the setting with a single surgical procedure type, it is convenient to use

$$
\begin{aligned}
\rho_{1}^{N} & =\frac{\mu_{1}^{N}}{\bar{\sigma}_{1}^{N}}, \\
\rho_{2}^{S} & =\frac{\mu_{2}^{S}}{\bar{\sigma}_{2}^{S}} .
\end{aligned}
$$

Note that in this setting, i.e., with two asymmetric procedure types in the absence of urgent procedures, the mean and standard deviation of the number of occupied beds have much simpler forms that can be written in terms of $\mu_{i}^{N}$ and $\bar{\sigma}_{1}^{N}$ :

$$
\begin{aligned}
M^{N}\left(\mathbf{a}^{e}\right) & =a_{1}^{e} \mu_{1}^{N}+a_{2}^{e} \mu_{2}^{N}, \\
\Sigma^{N}\left(\mathbf{a}^{e}\right) & =\bar{\sigma}_{1}^{N} a_{1}^{e} .
\end{aligned}
$$


The following result describes the composition of such a portfolio in the setting where all procedures are elective and the revenue the hospital receives is proportional to the expected load that a procedure exerts on hospital resources. For a proportional asymmetric setting in the absence of urgent procedures, define the following:

$$
\omega_{N, S}= \begin{cases}\sqrt{\frac{2}{\pi}} \frac{\pi^{S}}{\rho_{2}^{S} r_{S}}+\frac{\pi^{N}}{r^{N}} \operatorname{erfc}\left(\sqrt{\ln \left(\frac{\pi^{N} \rho_{2}^{S} r_{S}}{\pi^{S} \rho_{1}^{N} r_{N}}\right)}\right)+\frac{\pi^{S}}{r^{S}}, & \frac{\pi^{S}}{\rho_{2}^{S} r_{S}}<\frac{\pi^{N}}{\rho_{1}^{N} r_{N}}, \\ \sqrt{\frac{2}{\pi}} \frac{\pi^{N}}{\rho_{1}^{N} r_{N}}+\frac{\pi^{S}}{r^{S}} \operatorname{erfc}\left(\sqrt{\ln \left(\frac{\pi^{S} \rho_{1}^{N} r_{N}}{\pi^{N} \rho_{2}^{S} r_{S}}\right)}\right)+\frac{\pi^{N}}{r^{N}}, & \frac{\pi^{S}}{\rho_{2}^{S} r_{S}}>\frac{\pi^{N}}{\rho_{1}^{N} r_{N}} .\end{cases}
$$

Proposition 4. In the asymmetric setting with the absence of urgent procedures, the following statements hold.

(a) $\Pi\left(a_{1}^{e}, a_{2}^{e}\right)$ is a concave function of $\left(a_{1}^{e}, a_{2}^{e}\right)$ for $a_{1}^{e}, a_{2}^{e} \geq 0$.

(b) In the proportional asymmetric setting with

$$
\omega_{N, S} \geq 2,
$$

let $Z$ be the unique solution to

$$
\frac{1}{\sqrt{2 \pi}} e^{-\frac{1}{2} Z}=1-\frac{\pi^{N}}{2 r^{N}} \operatorname{erfc}\left(\sqrt{\frac{1}{2} Z+\ln \left(\frac{\pi^{N}}{\rho_{1}^{N} r_{N}}\right)}\right)-\frac{\pi^{S}}{2 r^{S}} \operatorname{erfc}\left(\sqrt{\frac{1}{2} Z+\ln \left(\frac{\pi^{S}}{\rho_{2}^{S} r_{S}}\right)}\right),
$$

and

$$
\begin{aligned}
& m^{-}=\frac{r^{N}}{r^{S}} \frac{1}{\left(1+\sqrt{Z+2 \ln \left(\frac{\pi^{N}}{\rho_{1}^{N} r_{N}}\right)} \frac{1}{\rho_{1}^{N}}\right)}, \\
& m^{+}=\frac{r^{N}}{r^{S}}\left(1+\sqrt{Z+2 \ln \left(\frac{\pi^{S}}{\rho_{2}^{S} r_{S}}\right)} \frac{1}{\rho_{2}^{S}}\right) .
\end{aligned}
$$

In addition, for

$$
m^{-}<\frac{\tau}{c}<m^{+}
$$

let

$$
\begin{aligned}
& a_{1}^{0}=\frac{1}{\mu_{1}^{S}} \frac{c m^{+}-\tau}{\frac{m^{+}}{m^{-}}-1} \\
& a_{2}^{0}=\frac{1}{\mu_{2}^{N}} \frac{\frac{\tau}{m^{-}}-c}{\frac{m^{+}}{m^{-}}-1} .
\end{aligned}
$$

Then the optimal portfolio, $\left(\hat{a}_{1}^{e}, \hat{a}_{2}^{e}\right)$, is determined as follows:

1. If $a_{1}^{0} \leq U_{1}$ and $a_{2}^{0} \leq U_{2}$, then $\left(\hat{a}_{1}^{e}, \hat{a}_{2}^{e}\right)=\left(a_{1}^{0}, a_{2}^{0}\right)$. 
2. If $a_{1}^{0} \leq U_{1}$ and $a_{2}^{0}>U_{2}$, then $\hat{a}_{2}^{e}=U_{2}$, and $\hat{a}_{1}^{e}=\min \left\{U_{1}, \hat{a}_{1}^{0}\right\}$, where

$$
\hat{a}_{1}^{0}=\frac{c-U_{2} \mu_{2}^{N}}{\mu_{1}^{N}+\bar{\sigma}_{1}^{N} \sqrt{Z_{1}}}
$$

and $Z_{1}$ is the unique solution to:

$$
\frac{\pi^{N} \bar{\sigma}_{1}^{N}}{\sqrt{2 \pi} R_{1}} e^{-\frac{1}{2} Z_{1}}=1-\frac{\pi^{N}}{2 r^{N}} \operatorname{erfc}\left(\sqrt{Z_{1}}\right)-\frac{\pi^{S}}{2 r^{S}} \operatorname{erfc}\left(\frac{1}{\bar{\sigma}_{2}^{S}}\left(\frac{\tau}{U_{2}}-\mu_{2}^{S}-\frac{\mu_{1}^{S}\left(c / U_{2}-\mu_{2}^{N}\right)}{\mu_{1}^{N}+\bar{\sigma}_{1}^{N} \sqrt{Z_{1}}}\right)\right) .
$$

3. If $a_{1}^{0}>U_{1}$ and $a_{2}^{0} \leq U_{2}$, then $\hat{a}_{1}^{e}=U_{1}$, and $\hat{a}_{2}^{e}=\min \left\{U_{2}, \hat{a}_{2}^{0}\right\}$, where

$$
\hat{a}_{2}^{0}=\frac{\tau-U_{1} \mu_{1}^{S}}{\mu_{2}^{S}+\bar{\sigma}_{2}^{S} \sqrt{Z_{2}}}
$$

and $Z_{2}$ is the unique solution to:

$$
\frac{\pi^{S} \bar{\sigma}_{2}^{S}}{\sqrt{2 \pi} R_{2}} e^{-\frac{1}{2} Z_{2}}=1-\frac{\pi^{N}}{2 r^{N}} \operatorname{erfc}\left(\frac{1}{\bar{\sigma}_{1}^{N}}\left(\frac{c}{U_{1}}-\mu_{1}^{N}-\frac{\mu_{2}^{N}\left(\tau / U_{1}-\mu_{1}^{S}\right)}{\mu_{2}^{S}+\bar{\sigma}_{2}^{S} \sqrt{Z_{2}}}\right)\right)-\frac{\pi^{S}}{2 r^{S}} \operatorname{erfc}\left(\sqrt{Z_{2}}\right) .
$$

4. If $a_{1}^{0}>U_{1}$ and $a_{2}^{0}>U_{2}$, then $\left(\hat{a}_{1}^{e}, \hat{a}_{2}^{e}\right)=\left(U_{1}, U_{2}\right)$.

Proposition 4 expresses the optimal numbers of procedures of two types in terms of easy-to-compute quantities $Z, Z_{1}$, and $Z_{2}$ that describe the overall load on hospital resources offered by the entire portfolio. Note that the bound (69) ensures that the expected duration of the procedures included in the portfolio does not exceed the nominal daily OR capacity $\tau$, while the expected number of beds occupied by patients recovering from all procedures does not exceed the nominal bed capacity $c$. Thus, the bound (69) is not restrictive as it is likely to be satisfied in any practical setting. The limits on the ratio of the capacities of two resources imposed by (73) ensure that the optimal portfolio includes positive values for the numbers of procedures of each type, and reflect a requirement that the hospital's patient treatment capacity is balanced: in other words, that the OR capacity and the recovery bed capacity are not mismatched.

From the expressions given by (71) and (72), we expect $m^{-}$to be decreasing in $\bar{\sigma}_{1}^{N}$ and $m^{+}$to be increasing in $\bar{\sigma}_{2}^{S}$. Then, expressions (74) and (75) suggest that $\hat{a}_{1}^{0}$ is increasing in $\bar{\sigma}_{2}^{S}$, while $\hat{a}_{2}^{0}$ is increasing in $\bar{\sigma}_{1}^{N}$. Hence, the variability of a resource usage of one procedure type not only affects its own "share" in the optimal portfolio but also the "share" of the other procedure type.

Figure 6 provides an example of how the composition of the optimal portfolio changes as a function of the recovery bed capacity within the range outlined by (73) in the absence (Figure 6a) and in the presence (Figure 6b) of the demand constraints. Recall that under the asymmetric proportional setting, two types of procedures share the same degree of "monetary attractiveness" as expressed by the revenue per unit of time each resource is used, $r^{N}$ and $r^{S}$. Thus, the features that distinguish two procedure types and, in the absence of demand restrictions, shape the competition for hospital service capacity are the different degrees of uncertainty the procedures display with 


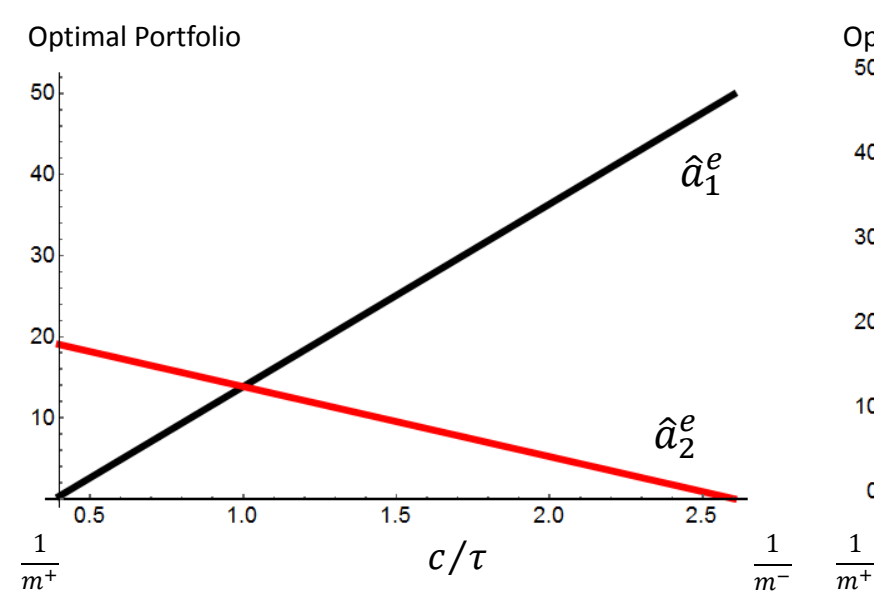

(a) No demand constraints

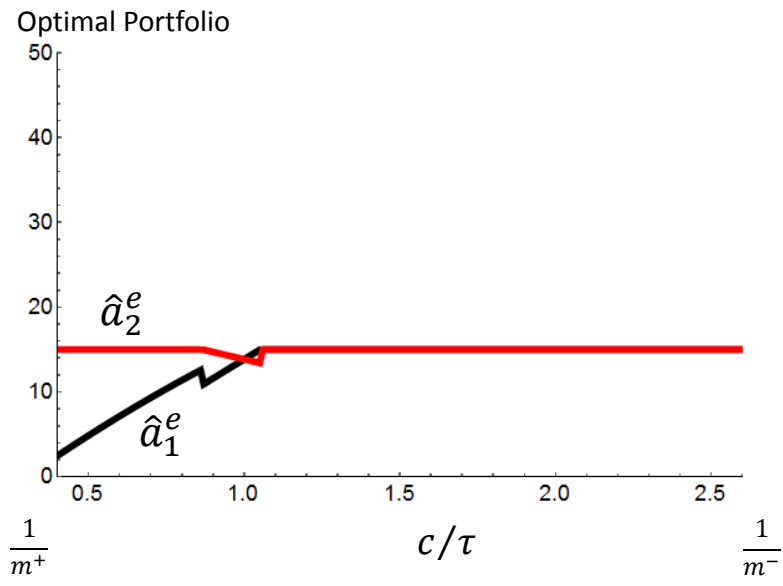

(b) $U_{1}=U_{2}=15$

Figure 6: The optimal portfolio for the asymmetric proportional setting in the absence of urgent procedures as a function of the number of recovery beds $c\left(r^{S}=r^{N}=R_{1}=R_{2}=1, \pi^{S}=\pi^{N}=1, \rho_{2}^{S}=\rho_{1}^{N}=0.5, \tau=50\right)$

respect to the utilization of hospital resources. From that perspective, procedures of type 2, which offer complete predictability in terms of the number of beds they require, are more preferable from the point of view of bed capacity utilization than the type- 1 procedures that provide the same compensation rate, but inject a degree of uncertainty in the way they use recovery beds. In the same way, taken in isolation, limited OR capacity will make type-1 procedures more preferred than type-2 procedures. The way this trade-off is resolved for any particular combination of OR capacity $\tau$ and bed capacity $c$ depends, among other things, on the parameters $\rho_{1}^{N}$ and $\rho_{2}^{S}$ that express the degrees of stochasticity that type-1 procedures bring in their utilization of bed capacity and type-2 procedures bring in their utilization of the OR capacity, respectively. In particular, when the recovery bed capacity is ample, the "front-end" approach to hospital capacity utilization will be optimal and will produce a portfolio that includes only type- 1 procedures, as indicated by the results for $\frac{c}{\tau}=\frac{1}{m^{-}}$on Figure 6a. On the other hand, the opposite is true of the optimal portfolio for the case of ample OR capacity: it will include only type- 2 procedures, as shown on Figure 6 a for $\frac{c}{\tau}=\frac{1}{m^{+}}$. For the intermediate values of $\frac{c}{\tau} \in\left(\frac{1}{m^{+}}, \frac{1}{m^{-}}\right)$both procedure types are "in play" as the impact of stochastic LOS durations from type-1 procedures is commensurate with that of the stochastic procedure durations of type-2 procedures.

The presence of limitations on the demand for surgical procedures may qualitatively alter the composition of the optimal portfolio of elective procedures, as indicated in Figure 6b. In addition to discontinuities in the portfolio composition outlined in Proposition 4 and observed at the points where one of the procedures reaches its upper demand limit, the presence of limited demand for a particular procedure allows the competing procedure to serve as a "replacement" and increase its 
presence in the portfolio. In the setting illustrated in Figure 6b, this effect is especially dramatic when the hospital operates sufficient number of beds, and severe demand restrictions make it profitable for the hospital to attract maximum possible number of both procedures, rather than focusing exclusively on the procedure that uses the "bottleneck" resource, i.e., OR time, in the most efficient manner.

\section{Allocation of Hospital Service Capacity: A Numerical Study}

In this section we use real data on the duration of surgical procedures as well as the resulting LOS values to illustrate the properties of optimal portfolios of elective surgical procedures. We also investigate the effectiveness of the "front-end" approach to allocating hospital service capacity in different real-life settings.

We collected the duration and LOS data at a medium-size teaching hospital. This data source covers the total of 71,030 surgical procedures, and corresponds to all procedures (excluding those related to vulnerable populations) performed at the hospital between January 1, 2014 and December 31, 2016. For each procedure, we record the surgery duration, patient LOS, and whether the surgery was elective. To account for the profitability of surgical procedures, we use the 2009 Nationwide Inpatient Sample (Healthcare Cost and Utilization Project 2013), the largest publicly available all-payer dataset for inpatient care in the U.S.

\subsection{Description of the data}

For our numerical analysis, we select the data for the most frequent majority-elective inpatient procedures. In particular, we focus on procedures with mean and median patient LOS of at least one day and with at least $50 \%$ of surgeries being elective (additional details on these procedures are provided in Section C.1 of the Appendix).

In order to provide a concise illustration of the properties of the optimal portfolios and the performance of the "front-end" capacity management approach, we picked the three most frequently performed procedures, which account for $17.28 \%$ of the cases in the subset of majority-elective inpatient procedures. Table 1 reports the average daily number of elective and nonelective procedures, OR time, and bed requirement parameters, as well as the hospital charges for these three procedures (the hospital charges are shown as the fraction of the charge for procedure 2 , the one with the highest charge).

It is important to note that the profit margin for a procedure is equal, on average, to a fraction of the hospital charges. In practice, while there may be some variation in the reimbursement fraction for different procedures depending on the type of payer that reimburses the hospital (government agency or private insurance company), we assume that the value of that fraction is approximately 


\begin{tabular}{|c|c|c|c|c|c|c|c|}
\hline \multirow{2}{*}{ Procedure, $i$} & \multicolumn{2}{|c|}{ Average Daily Number } & \multicolumn{2}{c|}{ Duration (Hours) } & Bed Requirement & Hospital Charges \\
\cline { 2 - 7 } & Elective & Non-Elective & Mean, $\mu_{i}^{S}$ & $\mathrm{SD}, \sigma_{i}^{S}$ & Mean, $\mu_{i}^{N}$ & $\mathrm{SD}, \sigma_{i}^{N}$ & (Relative to Procedure 2) \\
\hline 1 & 1.07 & 0.17 & 3.20 & 1.78 & 4.31 & 1.35 & 0.76 \\
\hline 2 & 0.77 & 0.18 & 4.38 & 2.27 & 5.80 & 1.50 & 1.00 \\
\hline 3 & 0.75 & 0.03 & 2.90 & 1.24 & 2.55 & 1.07 & 0.47 \\
\hline
\end{tabular}

Table 1 Average daily numbers, OR time and bed requirements, and hospital charges for the top three surgical procedures.

\begin{tabular}{|c|c|}
\hline \multicolumn{2}{|c|}{ Procedure 1} \\
\hline Team, $k$ & $q_{1, k}$ \\
\hline 1 & 0.3607 \\
\hline 2 & 0.1782 \\
\hline 3 & 0.1328 \\
\hline 4 & 0.0907 \\
\hline 5 & 0.0853 \\
\hline 6 & 0.0810 \\
\hline 7 & 0.0713 \\
\hline
\end{tabular}

\begin{tabular}{|c|c|}
\hline \multicolumn{2}{|c|}{ Procedure 2} \\
\hline Team, $k$ & $q_{2, k}$ \\
\hline 1 & 0.5325 \\
\hline 2 & 0.2062 \\
\hline 3 & 0.0989 \\
\hline 4 & 0.1624 \\
\hline
\end{tabular}

\begin{tabular}{|c|c|}
\hline \multicolumn{2}{|c|}{ Procedure 3} \\
\hline Team, $k$ & $q_{3, k}$ \\
\hline 1 & 0.3731 \\
\hline 2 & 0.1986 \\
\hline 3 & 0.1330 \\
\hline 4 & 0.0915 \\
\hline 5 & 0.0812 \\
\hline 6 & 0.1226 \\
\hline
\end{tabular}

Table 2 Fractions $q_{i k}$ of procedure $i=1,2,3$ performed by team $k$.

the same for all procedures. Thus, we will be using the charge value reported in Table 1 as a proxy for the profitability, $R_{i}$ of a particular procedure $i$. Also, as Table 1 indicates, all three procedures are performed predominantly on an elective basis, with a small nonelective component.

There are multiple teams that perform each procedure. For example, procedure 1 is performed by seven teams, while procedure 2 is performed by four teams. Table 2 reports the average fraction of each procedure performed by a particular surgical team.

In our numerical study we use the "base case" setting: Table 3 provides a summary of the "basecase" parameters, including the optimal portfolio values $\hat{a}_{i}^{e}, i=1, \ldots, 3$, for the three-procedure setting. Note that we continue to use continuous quantities to describe the numbers of surgical procedures. The estimation procedure used to obtain the parameters for this base case is described in detail in Section C.2 of the Appendix.

\begin{tabular}{|c|c|c|}
\hline \multicolumn{2}{|l|}{ Quantity } & Value \\
\hline \multicolumn{2}{|l|}{$\tau$, hours } & 14.79 \\
\hline \multicolumn{2}{|l|}{$c$, beds } & 18.00 \\
\hline \multicolumn{2}{|l|}{$\pi_{b}^{S}$, multiples of $R_{2}$} & 0.55 \\
\hline \multicolumn{2}{|l|}{$\pi_{b}^{N}$, multiples of $R_{2}$} & 14.03 \\
\hline \multirow{3}{*}{$\begin{array}{l}\text { Optimal daily number } \\
\text { of elective } \\
\text { procedures }\end{array}$} & Type-1, $\hat{a}_{1}^{e}$ & 1.55 \\
\hline & Type-2, $\hat{a}_{2}^{e}$ & 0.49 \\
\hline & Type- $3, \hat{a}_{3}^{e}$ & 0.87 \\
\hline
\end{tabular}

Table 3 "Base-case" values for the three-procedure setting. 


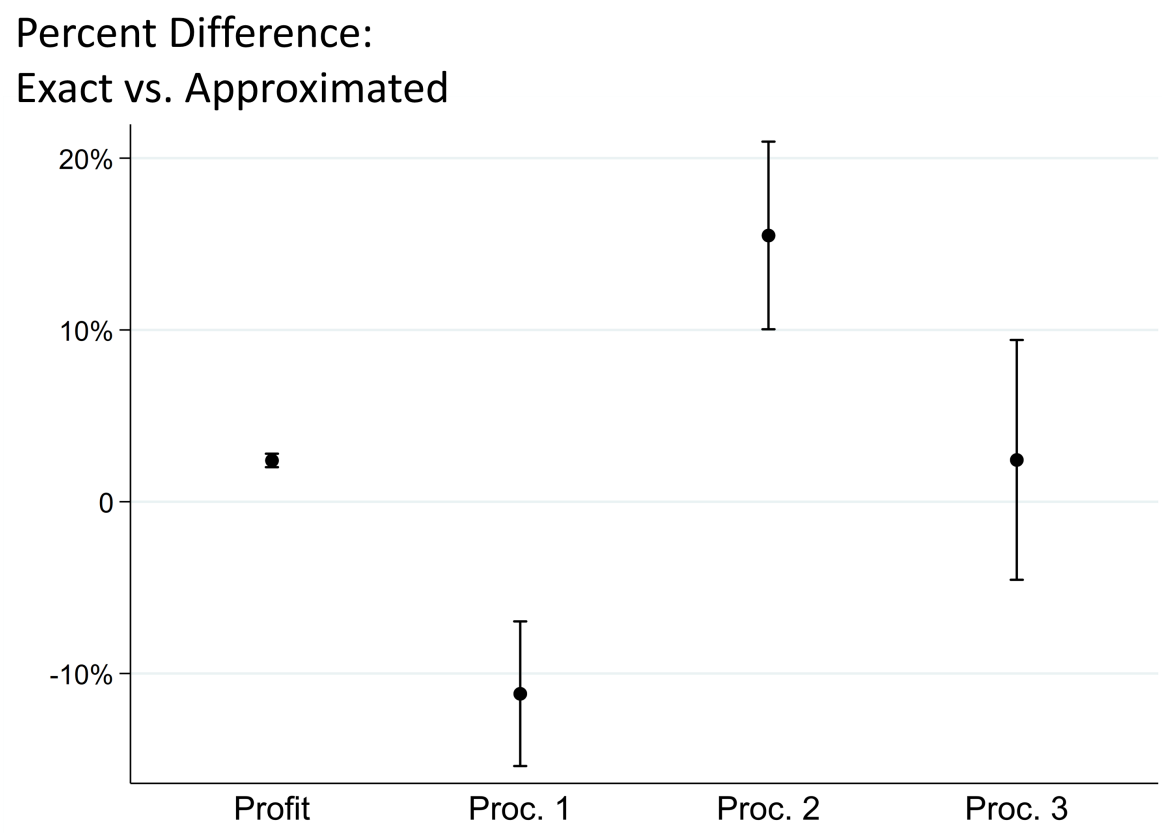

Figure 7 : Percentage difference between the exact and approximated values of the optimal profit and each of the three procedures in the optimal portfolio. The bars show the simulation-based $95 \%$ confidence intervals.

\subsection{Accuracy of the CLT-based approximation}

Our analysis of the optimal portfolios of elective procedures presented in Sections 4 and 5 is based on approximating the exact expected daily profit (19) by the expression given by (27). This approximation is based on the central-limit result of Proposition 1 that assumes that the number of physician teams performing every procedure is very high, and that, as stated in (24), none of the teams dominates others in terms of the proportion of procedures it performs. In the hospital example we studied, as specified in Table 2, the numbers of surgical teams that performed top three elective procedures was between 4 and 7. Thus, it is important to establish the accuracy of the approximation we used in our setting, i.e., the setting with small numbers of surgical teams.

In this section, we report the results of the numerical study that compares the optimal portfolio and corresponding profit obtained using (27) with their respective exact values defined by (19). The exact problem optimization using (19), similar to (27), involves evaluating an integral with a large number of combinations of different values of nonelective procedures. We evaluated this integral using Monte Carlo simulation by randomly drawing two sets of values: (1) LOS values according to their empirical distributions, and (2) the number of nonelective cases (the possible values are 0 or 1 with probabilities reported in column 3 of Table 1 ).

To compare the exact and the approximate approaches, we evaluated each approach using 50 different random seeds. Let $\hat{\mathbf{a}}_{E}^{e}$ be the vector representing the exact optimal portfolio, i.e., the 
portfolio that optimizes (19). Figure 7 shows the $95 \%$ confidence intervals for average percentage difference between the exact and the approximate values of the optimal portfolio and the corre-

sponding profit values. In particular, the percentage differences are calculated as $\frac{\tilde{\Pi}\left(\hat{\mathbf{a}}_{E}^{e}\right)-\Pi\left(\hat{\mathbf{a}}^{e}\right)}{\tilde{\Pi}\left(\hat{\mathbf{a}}_{E}^{e}\right)} \times 100$ for the optimal profit and as $\frac{\hat{a}_{i, E}^{e}-\hat{a}_{i}^{e}}{\hat{a}_{i, E}^{e}} \times 100$ for each procedure in the optimal portfolio.

The optimal portfolios suggested by the exact and approximate solutions are significantly different. In particular, the approximation appears to favor procedure 1 over procedure 2 in a statistically significant way, with the average differences of about 10\%-15\%. However, these different portfolios generate very close daily expected profits, since the approximated optimal profit is within $5 \%$ of the exact value. Moreover, the approximation qualitatively maintains the ordering of procedures in the portfolio: in both the approximated and exact solutions, procedures 1 and 2 have the highest and lowest optimal values, respectively.

We also investigate the impact of the penalty costs, $\pi^{N}$ and $\pi^{S}$, on the performance of our approximation in Section C.3 of the Appendix (Figures C1-C3). We observe that as the values of $\pi^{N}$ and $\pi^{S}$ change by $50 \%$ in either direction, the approximated optimal profit stays within $5 \%$ of its exact value. All these observations suggest that the approximation-based optimal portfolios perform reasonably well as compared to the exact ones.

\subsection{Optimal portfolio composition and the performance of the "front-end" capacity management approach}

In our numerical study we focus on illustrating how the key outcomes are affected by the penalty $\operatorname{costs}\left(\pi^{N}\right.$ and $\left.\pi^{S}\right)$, variability of procedure bed occupancy and OR time $\left(\left(\sigma_{i}^{N}\right)^{2}\right.$ and $\left.\left(\sigma_{i}^{S}\right)^{2}\right)$, and hospital bed capacity $(c)$. In particular, we look separately at the impact of these five parameters on (1) the optimal portfolio composition and utilization of hospital resources, and (2) the performance gap of the front-end approach.

Table 1 reports the profit margin as well as the expected value and standard deviation for procedure duration and bed requirements corresponding to each of the three procedure types. In order to gain insights into the composition of the optimal portfolio of elective procedures, it is useful to express the data in Table 1 using the "profitability ratios" for each procedure with respect to how it utilizes each hospital resource. Table 4 shows these parameters defined as the ratios of the profit margin to the first as well as the second moments of the requirements that each procedure presents for each resource.

As Table 4 indicates, procedures 1 and 2 are similar in terms of how profitable they are given how much of each resource (OR time and bed capacity) they consume. Procedure 3 stands separately from procedures 1 and 2 . On the one hand, it appears to be a less attractive choice with respect to the utilization of OR time, as it is dominated by the first two procedures, requiring, on a "dollar for 


\begin{tabular}{|c|l|l|l|l|}
\hline \multirow{2}{*}{ Procedure, $i$} & \multicolumn{2}{|c|}{ Duration } & \multicolumn{2}{c|}{ Bed Requirement } \\
\cline { 2 - 5 } & $R_{i} / \mu_{i}^{S}$ & $R_{i} / \sigma_{i}^{S}$ & $R_{i} / \mu_{i}^{N}$ & $R_{i} / \sigma_{i}^{N}$ \\
\hline 1 & 0.2375 & 0.4270 & 0.1763 & 0.5630 \\
\hline 2 & 0.2283 & 0.4405 & 0.1724 & 0.6667 \\
\hline 3 & 0.1621 & 0.3790 & 0.1843 & 0.4393 \\
\hline
\end{tabular}

Table 4 Profitability ratios for the top three surgical procedures.

dollar" basis, more OR time on average, and is more unpredictable in its OR utilization. However, in terms of bed utilization the preference ordering among the three procedure types is less clear: procedure 3 is more attractive from the point of view of the expected compensation for bed usage, but appears to result in a disproportionately high standard deviation of bed usage.

6.3.1. Impact on optimal portfolio and hospital resources: We investigate the impact of changes in $\pi^{N}, \pi^{S},\left(\sigma_{1}^{N}\right)^{2},\left(\sigma_{1}^{S}\right)^{2}$, and $c$ on four key quantities: a) expected bed utilization, b) expected OR utilization, c) procedure components of elective bed utilization, and d) procedure components of elective OR utilization. In particular, we define the expected bed utilization as

$$
u_{c}=\frac{\sum_{i=1}^{3} \mu_{i}^{N}\left(\hat{a}_{i}^{e}+a_{i}^{u}\right)}{c},
$$

and the expected OR utilization as

$$
u_{\tau}=\frac{\sum_{i=1}^{3} \mu_{i}^{S}\left(\hat{a}_{i}^{e}+a_{i}^{u}\right)}{\tau} .
$$

Further, the component of elective bed utilization corresponding to procedure $i=1, \ldots, 3$ is defined as

$$
x_{i}^{c}=\frac{\mu_{i}^{N} \hat{a}_{i}^{e}}{\sum_{j=1}^{3} \mu_{j}^{N} \hat{a}_{j}^{e}},
$$

and the component of elective OR utilization corresponding to procedure $i=1, \ldots, 3$ is defined as

$$
x_{i}^{\tau}=\frac{\mu_{i}^{S} \hat{a}_{i}^{e}}{\sum_{j=1}^{3} \mu_{j}^{S} \hat{a}_{j}^{e}} .
$$

Note that (82) and (83) reflect the contributions of elective procedures of each type to the total expected utilization of the corresponding resource by the elective procedures. We place all the figures of this section in Section C.4, and only summarize the insights generated by them here. In the figures we change one of the parameters, while all others remain at their base case levels.

We observe the impact of penalty costs, $\pi^{N}$ and $\pi^{S}$, while they change over the ranges $\frac{\pi^{N}}{\pi_{b}^{N}} \in$ $(0,1.5)$, and $\frac{\pi^{S}}{\pi_{b}^{S}} \in(0.6,2)$ (Figures $\mathrm{C} 4$ and $\mathrm{C} 5$ ). When one of the penalties associated with exceeding 
hospital's nominal resource capacities is increased, the expected utilizations of both resources decrease. For low values of $\pi^{N}$, on the other hand, both expected utilizations exceed $100 \%$ because the penalty cost $\pi_{b}^{S}$ associated with exceeding the nominal capacity of the OR capacity is not sufficiently large. Note that we do not observe such a phenomena when $\pi^{S}$ is low.

Our analysis highlights the fact that the composition of optimal portfolio depends on how "good" each procedure is in using both OR time and hospital beds. For example, were recovery beds the only hospital resource, there would be a clear preference for procedure 3 because it is the procedure that most profitably utilizes bed capacity, in expectation. However, procedures 1 and 2 are better than procedure 3 at utilizing OR capacity, on average. Thus, procedure 3 yields its portfolio share to procedures 1 and 2 for lower values of $\pi^{N}$ or for higher values of $\pi^{S}$. In the extreme case, where the value of $\pi^{N}$ is close to 0 , procedure 3 is completely left out of the portfolio. On the other hand, procedure 3 remains in the optimal portfolio for all values of $\pi^{S}$ due to the presence of the second constraining resource, recovery beds.

When examining the impact of changes in the variability of LOS and OR time on the four key quantities defined above, we change the parameters of the distributions of OR time and bed occupancy for procedure 1 (Figures C6 and C7). In particular, we keep the means of OR time and bed occupancy constant and scale their variances, e.g., $\mu_{1^{\prime}}^{N}=\mu_{1}^{N}$ and $\left(\sigma_{1^{\prime}}^{N}\right)^{2}=\kappa\left(\sigma_{1}^{N}\right)^{2}$, where $0.5 \leq \kappa \leq 1.5$. The details of this scaling procedure are presented in Section C.4 of the Appendix.

As the variability of bed occupancy for procedure 1 increases, the portfolio "share" allocated to procedure 1 goes down. We also observe that as $\left(\sigma_{1}^{N}\right)^{2}$ increases, bed utilization decreases and OR utilization increases. Similarly, the "share" of procedure 1 in the optimal portfolio decreases as $\left(\sigma_{1}^{S}\right)^{2}$ increases. However, the OR utilization first decreases and then increases in $\left(\sigma_{1}^{S}\right)^{2}$, while the bed utilization is always decreasing.

Changes in the expected OR utilization highlight the subtle, yet consequential, relationships between this outcome and key system variables such as a procedure's variances of OR time or bed occupancy. The underlying reason for these variances to have non-obvious impact on OR utilization is portfolio re-balancing; e.g., if the variance of OR time for procedure 1 increases, the optimal portfolio will likely favor the other procedures. The resulting OR utilization may then increase or decrease, depending on the characteristics of the procedures that replace procedure 1 in the new optimal portfolio.

Finally, we investigate the effect of the number of beds, $c$, for $c \in\{14,15, \ldots, 22\}$ (Figure C8). The utilization of beds and OR time both increase with bed capacity. Also, as bed capacity increases, the portfolio share of procedure 3- the best procedure of the three in terms of profitably utilizing bed capacity - decreases. This is because increases in bed capacity lead to OR time becoming more of a bottleneck resource, and procedures 1 and 2 are better than procedure 3 in terms of profitably 
utilizing OR time (Table 4). Note that the overall impact of increasing $c$ is close to the impact of decreasing $\pi^{N}$.

6.3.2. Impact on the performance of the front-end policy This section considers the impact of changes in $\pi^{N}, \pi^{S},\left(\sigma_{1}^{N}\right)^{2},\left(\sigma_{1}^{S}\right)^{2}$, and $c$, respectively, on the performance gap of the "frontend" approach to hospital capacity management (Figure C9). This quantity is defined similarly to the expression in (57):

$$
\Delta_{S}=\frac{\Pi\left(\hat{\mathbf{a}}^{e}\right)-\Pi\left(\hat{\mathbf{a}}_{S}^{e}\right)}{\Pi\left(\hat{\mathbf{a}}^{e}\right)},
$$

where for every combination of the penalty $\operatorname{costs}\left(\pi^{S}, \pi^{N}\right), \hat{\mathbf{a}}^{e}=\left(\hat{a}_{1}^{e}, \hat{a}_{2}^{e}, \hat{a}_{3}^{e}\right)$ is the optimal portfolio of surgical procedures, and $\hat{\mathbf{a}}_{S}^{e}=\left(\hat{a}_{1, S}^{e}, \hat{a}_{2, S}^{e}, \hat{a}_{3, S}^{e}\right)$ is the optimal portfolio of surgical procedures for a given value of $\pi^{S}$ and for $\pi^{N}=0$.

At the base case, the front-end policy that ignores the impact of the elective portfolio on bed capacity leads to a substantial profit loss, i.e., $\Delta_{S}=11.7$, as a consequence of a relatively small value of the "base case" penalty cost $\pi_{b}^{S}$. This is because the portfolio that is selected at $\pi^{N}=0$ is considerably different from the one with non-zero $\pi^{N}$, e.g., procedure 3 is not in the portfolio when $\pi^{N}=0$ (Figure C4c). Also, the bed utilization exceeds $100 \%$ for near-zero values of $\pi^{N}$ (Figure C4a), which leads to substantial revenue loss as $\pi^{N}$ increases unless the portfolio is rebalanced. The profit loss associated with the front-end policy, while mitigated by the increases in the penalty $\operatorname{cost} \pi^{S}$, persists even when this penalty doubles as compared to the "base case".

The variance of bed occupancy and OR time for procedure 1 affect the performance gap in opposite directions. This is partly because the variance of bed occupancy has no effect on the optimal portfolio of the front-end approach. As bed capacity increases, the key constraining resource becomes the operating room, which is the only resource considered in the front-end approach. Accordingly, the performance of the front-end approach approaches optimality in this case.

Finally, Figure C10 presents the trade-off between the variance of bed occupancy and OR time. In this plot, all the points on the line have the same front-end performance gap, equal to the baseline gap of about 11.7. Also, the plot passes through $(1,1)$ by construction because both variance values are set to their empirical values. The figure shows a "budget set of uncertainty" that results in the same front-end performance gap: increases in procedure 1's bed occupancy variance can be compensated for by reductions in the procedure's OR time variance.

\section{Discussion}

Hospitals are complex systems that serve a variety of patients, each using multiple resources. To operate effectively, patient needs should be strategically matched with the available resource capacity. In this paper, we build a model that combines the task of rationing limited, "front-end" 
OR capacity with allocation of the "downstream" bed capacity. Our study focuses on building analytical insights into the impact of stochastic procedure and LOS durations on the trade-offs among multiple procedure types. Our model accommodates arbitrary finite-support distributions and uses a CLT-based two-moment approximation for the total procedure duration and the daily number of occupied beds for a given portfolio of procedures.

Our analysis focuses on the strategic match between the demand for surgical procedures and the supply of hospital service capacity. We make several simplifying assumptions. First, we treat the hospital bed capacity as homogeneous, whereas in practice it is likely that different procedure types, while "competing" for the common OR capacity, may require different types of beds at the recovery stage. In addition, we assume that the hospital uses the same portfolio of surgical procedures every day, while in reality a weekly scheduling cycle is more common, reflecting the dependence of both available resources and surgeons' preferences on the day of the week. In such settings, our model can be generalized to handle the weekly cycle of procedures by formulating the problem's objective function using separable (by day of the week) OR capacity terms, and a "mixed" recovery bed term that, for every day, may contain the variables corresponding to the procedures performed on all days of the week.

Another potential line of work to complement our approach here would focus on mechanisms of tactical management of constrained hospital resources. In our model, we use an "open loop" approach to construct the optimal procedure mix and do not utilize knowledge of actual bed occupancy to adjust the influx of already scheduled procedures in situations with looming bed shortage. In practice, hospitals often use rescheduling and/or cancellations of elective procedures as one of the tools for restoring the tactical balance between the demand for recovery services and the supply of beds. The analysis of these dynamic cancellation and rescheduling policies is a challenging and promising extension to the present work.

\section{References}

Adan I, Bekkers J, Dellaert N, Vissers J, Yu X (2009) Patient mix optimisation and stochastic resource requirements: A case study in cardiothoracic surgery planning. Health Care Management Science 12(2):129-141.

Adan I, Vissers J (2002) Patient mix optimisation in hospital admission planning: a case study. International Journal of Operations and Production Management 22(4):445-461.

Altman E, Jiménez T, Koole G (2001) On optimal call admission control in a resource-sharing system. IEEE Transactions on Communications 49(9):1659-1668.

Ayvaz N, Huh WT (2010) Allocation of hospital capacity to multiple types of patients. Journal of Revenue and Pricing Management 9(5):386-398. 
Barz C, Rajaram K (2015) Elective patient admission and scheduling under multiple resource constraints. Production and Operations Management 24(12):1907-1930.

Begen M, Queyranne M (2011) Appointment scheduling with discrete random durations. Mathematics of Operations Research 36(2):240-257.

Bekker R, Koeleman P (2011) Scheduling admissions and reducing variability in bed demand. Health Care Management Science 14(3):237-249, ISSN 1386-9620.

Beliën J, Demeulemeester E (2007) Building cyclic master surgery schedules with leveled resulting bed occupancy. European Journal of Operational Research 176(2):1185-1204.

Beliën J, Demeulemeester E, Cardoen B (2006) Visualizing the demand for various resources as a function of the master surgery schedule: A case study. Journal of Medical Systems 30(5):343-350.

Billingsley P (1995) Probability and Measure (Wiley, New York).

Bitran G, Mondschein S (1995) An application of yield management to the hotel industry considering multiple day stays. Operations Research 43(3):427-443.

Brickley J, Van Horn R (2002) Managerial incentives in nonprofit organizations: Evidence from hospitals. The Journal of Law and Economics 45(1):227-249.

Cardoen B, Demeulemeester E, Beliën J (2010) Operating room planning and scheduling: A literature review. European Journal of Operational Research 201(3):921-932.

Carr S, Lovejoy B (2000) The inverse newsvendor problem: Choosing an optimal demand portfolio for capacitated resources. Management Science 46(7):912-927.

Ciliberto F, Lindrooth R (2007) Exit from the hospital industry. Economic Inquiry 45(1):71-81.

Cohen M, Ho T, Ren Z, Terwiesch C (2003) Measuring imputed cost in the semiconductor equipment supply chain. Management Science 49(12):1653-1670.

Dai J, Shi P (2018) Inpatient overflow: An approximate dynamic programming approach. Manufacturing \& Service Operations Management (forthcoming).

De Bruin A, Bekker R, van Zanten L, Koole G (2010) Dimensioning hospital wards using the Erlang loss model. Annals of Operations Research 178(1):23-43, ISSN 0254-5330.

Deshpande V, Arıkan M (2012) The impact of airline flight schedules on flight delays. Manufacturing \& Service Operations Management 14(3):423-440.

Dindo D, Demartines N, Clavien PA (2004) Classification of surgical complications: A new proposal with evaluation in a cohort of 6336 patients and results of a survey. Annals of Surgery 240(2):205-213.

Duggan M (2000) Hospital ownership and public medical spending. The Quarterly Journal of Economics 115(4):1343-1373.

Essen J, Bosch J, Hans E, Houdenhoven M, Hurink J (2013) Reducing the number of required beds by rearranging the OR-schedule. OR Spectrum 1-21, ISSN 0171-6468. 
Foster T (2012) Data for benchmarking your ors performance. OR Manager 28(1):13-16.

Haider A, Obirieze A, Velopulos C, Richard P, Latif A, Scott V, Zogg C, Haut E, Efron D, Cornwell E, MacKenzie E, Gaskin D (2015) Incremental cost of emergency versus elective surgery. Annals of Surgery $262(2): 260-266$.

Healthcare Cost and Utilization Project (2013) Overview of the Nationwide Inpatient Sample (NIS). URL http://www.hcup-us.ahrq.gov/nisoverview.jsp, retrieved on May 21, 2017.

Helm J, AhmadBeygi S, Van Oyen M (2011) Design and analysis of hospital admission control for operational effectiveness. Production and Operations Management 20(3):359-374, ISSN 1937-5956.

Helm J, Van Oyen M (2014) Design and optimization methods for elective hospital admissions. Operations Research 62(6):1265-1282.

Hulshof PJ, Mes MR, Boucherie RJ, Hans EW (2016) Patient admission planning using approximate dynamic programming. Flexible Services and Manufacturing Journal 28(1-2):30-61.

Lai K, Ng W (2005) A stochastic approach to hotel revenue optimization. Computers and Operations Research 32(5):1059-1072.

Lee D, Zenios S (2012) An evidence-based incentive system for medicare's end-stage renal disease program. Management science 58(6):1092-1105.

Littlewood K (2005) Special issue papers: forecasting and control of passenger bookings. Journal of Revenue and Pricing Management 4(2):111-123.

National Center for Health Statistics (2017) Health, united states, 2016: With chartbook on long-term trends in health .

Nunes L, de Carvalho S, Rodrigues R (2009) Markov decision process applied to the control of hospital elective admissions. Artificial Intelligence in Medicine 47(2):159-171.

Olivares M, Terwiesch C, Cassorla L (2008) Structural estimation of the newsvendor model: an application to reserving operating room time. Management Science 54(1):41-55.

Örmeci E, Burnetas A (2005) Dynamic admission control for loss systems with batch arrivals. Advances in Applied Probability 37(4):915-937.

Örmeci E, Burnetas A, Wal V (2001) Admission policies for a two class loss system. Stochastic Models $17(4): 513-539$.

Papier F, Thonemann U (2010) Capacity rationing in stochastic rental systems with advance demand information. Operations Research 58(2):274-288.

Rath S, Rajaram K, Mahajan A (2017) Integrated anesthesiologist and room scheduling for surgeries: Methodology and application. Operations Research 65(6):1460-1478.

Savin S, Cohen M, Gans N, Katalan Z (2005) Capacity management in rental businesses with two customer bases. Operations Research 53(4):617-631. 
Song H, Tucker AL, Graue R, Moravick S, Yang JJ (2018) Capacity pooling in hospitals: The hidden consequences of off-service placement, Working Paper.

Talluri K, Van Ryzin G (2005) The Theory and Practice of Revenue Management (Springer Verlag).

Truong V, Wang X, Liu N, Anderson B (2013) Integrated scheduling and capacity planning with considerations for patients' length-of-stays, Working Paper.

US Government Accountability Office (2003) Specialty hospitals: Geographic location, services provided, and financial performance. Pub. no. GAO-04-167. URL http://www.gao.gov/new.items/d04167.pdf, retrieved on May 21, 2017.

Vanberkel P, Boucherie R, Hans E, Hurink J, van Lent W, van Harten W (2011) An exact approach for relating recovering surgical patient workload to the master surgical schedule. Journal of the Operational Research Society 62(10):1851-1860.

Wang J, Cabrera J, Tsui KL, Guo H, Bakker M, Kostis JB (2018) Clinical and non-clinical effects on surgery duration: Statistical modeling and analysis. URL https://arxiv.org/pdf/1801.04110.pdf, retrieved on January 29, 2018.

Weatherford L (1995) Length of stay heuristics: do they really make a difference? Cornell Hotel and Restaurant Administration Quarterly 36(6):70-79. 


\section{Appendices for "Managing Portfolio of Elective Surgical Procedures: A Multidimensional Inverse Newsvendor Problem"}

\section{Appendix A: Proofs of Analytical Results}

\section{Proof of Proposition 1}

Let

$$
\bar{S}_{i, k, t}=S_{i, j, t}, j \in C_{i, k}, k=1, \ldots, K_{i}
$$

denote the value of the type- $i$ procedure duration realized on day $t$ for any procedure $j$ in the subset $C_{i, k}$. Note that the marginal pdf for the distribution of $\bar{S}_{i, k, t}$ is given by

$$
p_{S}^{\mathrm{m}}\left(\bar{S}_{i, k, t}=s\right)=f_{i}^{S}(s)=\int_{0}^{L_{\max }^{i}} f_{i}(s, l) d l, s \in\left[0, S_{\max }^{i}\right], i=1, \ldots, n, t=1,2, \ldots, k=1, \ldots, K_{i}
$$

with

$$
\int_{0}^{S_{\max }^{i}} f_{i}^{S}(s) d s=1, i=1, \ldots, n
$$

and does not depend on $k$ or $t$.

The total duration of all surgical procedures of type $i$ performed on day $t$ is given by:

$$
D_{i, t}=a_{i, t} \mathcal{S}_{i, t}
$$

where

$$
\mathcal{S}_{i, t}=\sum_{k=1}^{K_{i}} q_{i k} \bar{S}_{i, k, t}
$$

is a convex combination of independent and identically distributed (iid) random variables $\bar{S}_{i, k, t}$. Note that $\mathcal{S}_{i, t}$ is a random variable with the support on $\left[0, S_{\max }\right]$ and the expectation and the variance given by $\mu_{i}^{\mathrm{S}}$ and $\left(\sigma_{i}^{\mathrm{S}}\right)^{2}\left(\sum_{k=1}^{K_{i}} q_{i k}^{2}\right)$, respectively. The Lyapunov Central Limit Theorem (Billingsley 1995, p. 362) states that for the sequence of $m$ independent random variables $X_{n}$, $n=1, \ldots, m$, each with the finite expectation $\mu_{n}$ and the variance $\sigma_{n}^{2}$, the expression

$$
\frac{\sum_{n=1}^{m}\left(X_{n}-\mu_{n}\right)}{\sqrt{\sum_{n=1}^{m} \sigma_{n}^{2}}}
$$

converges in distribution to a standard normal random variable $N(0,1)$ when $m \rightarrow \infty$ provided that there exists $\delta>0$ such that

$$
\lim _{m \rightarrow \infty} \frac{\sum_{n=1}^{m} E\left[\left|X_{n}-\mu_{n}\right|^{2+\delta}\right]}{\left(\sum_{n=1}^{m} \sigma_{n}^{2}\right)^{\frac{2+\delta}{2}}}=0 .
$$


Applying this result to

$$
X_{k}=q_{i k} \bar{S}_{i, k, t},
$$

we get $E\left[X_{k}\right]=q_{i k} \mu_{i}^{\mathrm{S}}, \operatorname{Var}\left[X_{k}\right]=q_{i k}^{2}\left(\sigma_{i}^{S}\right)^{2}$, so that the expression in (A7) becomes

$$
\begin{aligned}
& \frac{\sum_{k=1}^{K_{i}} E\left[\left|X_{k}-\mu_{k}\right|^{2+\delta}\right]}{\left(\sum_{k=1}^{K_{i}} \sigma_{k}^{2}\right)^{\frac{2+\delta}{2}}}=\left(\frac{\int_{0}^{S_{\max }} f_{i}^{S}(s)\left|s-\mu_{i}^{S}\right|^{2+\delta} d s}{\left(\sigma_{i}^{S}\right)^{2+\delta}}\right)\left(\frac{\sum_{k=1}^{K_{i}} q_{i k}^{2+\delta}}{\left(\sum_{k=1}^{K_{i}} q_{i k}^{2}\right)^{\frac{2+\delta}{2}}}\right) \\
= & \left(\frac{\int_{0}^{S_{\max }} f_{i}^{S}(s)\left|s-\mu_{i}^{S}\right|^{2+\delta} d s}{\left(\sigma_{i}^{S}\right)^{2+\delta}}\right)\left(\frac{\left(\sum_{k=1}^{K_{i}} q_{i k}^{2+\delta}\right)^{\frac{1}{2+\delta}}}{\left(\sum_{k=1}^{K_{i}} q_{i k}^{2}\right)^{\frac{1}{2}}}\right)^{2+\delta}
\end{aligned}
$$

The limit of the last expression when $K_{i} \rightarrow \infty$ is 0 if and only if

$$
\lim _{K_{i} \rightarrow \infty} \frac{\left(\sum_{k=1}^{K_{i}} q_{i k}^{2+\delta}\right)^{\frac{1}{2+\delta}}}{\left(\sum_{k=1}^{K_{i}} q_{i k}^{2}\right)^{\frac{1}{2}}}=0 .
$$

Note that, for (A8), we have

$$
\frac{\sum_{k=1}^{K_{i}}\left(X_{k}-\mu_{k}\right)}{\sqrt{\sum_{k=1}^{K_{i}} \sigma_{k}^{2}}}=\frac{\mathcal{S}_{i, t}-\mu_{i}^{S}}{\sigma_{i}^{S} \sqrt{\sum_{k=1}^{K_{i}} q_{i k}^{2}}} .
$$

Thus, as $K_{i} \rightarrow \infty$,

$$
\frac{\mathcal{S}_{i, t}-\mu_{i}^{S}}{\sigma_{i}^{S} \sqrt{\sum_{k=1}^{K_{i}} q_{i k}^{2}}} \stackrel{d}{\rightarrow} \mathcal{N}(0,1) .
$$

We can use this result to represent the limiting distribution of $\mathcal{S}_{i, t}$ for any $t$ as a normal random variable with the mean $\mu_{i}^{\mathrm{S}}$ and the standard deviation $\sigma_{i}^{S} \sqrt{\sum_{k=1}^{K_{i}} q_{i k}^{2}}$. Then, given the independence of procedure durations for different procedure types, the total duration of all surgical procedures performed on day $t$ is represented by a random variable,

$$
D_{t}\left(\mathbf{a}^{e}, \mathbf{a}_{t}^{u}\right)=\sum_{i=1}^{n} a_{i, t} \mathcal{S}_{i, t} \sim N\left(\sum_{i=1}^{n} a_{i, t} \mu_{i}^{S}, \sqrt{\sum_{i=1}^{n}\left(a_{i, t}\right)^{2}\left(\sigma_{i}^{S}\right)^{2}\left(\sum_{k=1}^{K_{i}} q_{i k}^{2}\right)}\right),
$$

i.e., a normal random variable with the mean $M^{S}\left(\mathbf{a}^{e}, \mathbf{a}_{t}^{u}\right)=\sum_{i=1}^{n} a_{i, t} \mu_{i}^{S}$ and the standard deviation $\Sigma^{S}\left(\mathbf{a}^{e}, \mathbf{a}_{t}^{u}\right)=\sqrt{\sum_{i=1}^{n}\left(a_{i, t}\right)^{2}\left(\bar{\sigma}^{\mathrm{S}_{i}}\right)^{2}}$, where $a_{i, t}=a_{i}^{e}+a_{i, t}^{u}$ and $\bar{\sigma}_{i}^{\mathrm{S}}$ is defined as $\sqrt{\left(\sigma_{i}^{S}\right)^{2}\left(\sum_{k=1}^{K_{i}} q_{i k}^{2}\right)}$ by $(10)$. 
Next we will apply a similar approach to modeling the number of beds $O_{t}\left(\mathbf{a}^{e}, \mathbf{a}_{t-L_{\max }}^{u}, \ldots, \mathbf{a}_{t}^{u}\right)$ occupied by recovering patients at midnight on day $t \geq L_{\max }^{i}, i=1, \ldots, n$ ("midnight census"). Under the correlation structure of Assumption 5 we can define

$$
\bar{L}_{i, k, t}=L_{i, j, t}, j \in C_{i, k}, k=1, \ldots, K_{i}
$$

to denote the value of the LOS duration resulting from a procedure of type $i$ performed on day $t$ for any procedure $j$ in the subset $C_{i, k}$. The marginal pdf for the distribution of $\bar{L}_{i, k, t}$ is given by

$$
p_{L}^{\mathrm{m}}\left(\bar{L}_{i, k, t}=l\right)=f_{i}^{L}(l)=\int_{0}^{S_{\max }^{i}} f_{i}(s, l) d s, l \in\left[0, L_{\max }^{i}\right], i=1, \ldots, n, t=1,2, \ldots, k=1, \ldots, K_{i}
$$

with

$$
\int_{0}^{L_{\max }^{i}} f_{i}^{L}(l) d l=1, i=1, \ldots, n,
$$

and, similar to $f_{i}^{S}(s)$, does not depend on $k$ or $t$. Similar to (5)-(6), we use

$$
\mu_{i}^{N}=\int_{0}^{L_{\max }^{i}} l f_{i}^{L}(l) d l=\int_{0}^{L_{\max }^{i}} \int_{0}^{S_{\max }^{i}} l f_{i}(s, l) d s d l
$$

and

$$
\left(\sigma_{i}^{L}\right)^{2}=\int_{0}^{L_{\max }} f_{i}^{L}(l)\left(l-\mu_{i}^{N}\right)^{2} d l=\int_{0}^{L_{\max }^{i}} \int_{0}^{S_{\max }^{i}}\left(l-\mu_{i}^{N}\right)^{2} f_{i}(s, l) d s d l
$$

to denote the expected value and the variance of the LOS duration resulting from a procedure of the $i$-th type. Note that the expression in (A17) is also the expected number of days a type- $i$ patient occupies a bed, so we denote it by $\mu_{i}^{N}$, rather than $\mu_{i}^{L}$.

Let $I_{i, k, t-T}$ be the indicator variable that is equal to 1 if patients who underwent procedure from a subset $k$ of type $i$ on day $t-T(T=0,1,2, \ldots)$ remain at the hospital and contribute to the midnight census on day $t$, and equal to 0 otherwise. Noting that $p_{i, T}^{L}$ is the probability that a type- $i$ patient stays at the hospital for at least $T$ days for $T=0, \ldots, L_{\max }^{i}$, which is defined by (9), we can express the probability that $I_{i, k, t-T}=1$ as

$$
P\left(I_{i, k, t-T}=1\right)= \begin{cases}p_{i, T}^{L}, & T=0, \ldots, L_{\max }^{i} \\ 0, & T \geq L_{\max }^{i}+1\end{cases}
$$

with $p_{i, L_{\max }^{i}}^{L}=0$. Note that $I_{i, k_{1}, t-T}$ and $I_{i, k_{2}, t-T}$ are i.i.d. random variables for any pair of $k_{1}, k_{2} \in$ $\left\{1,2, \ldots, K_{i}\right\}$ with $k_{1} \neq k_{2}$.

Using the indicator variables $I_{i, k, t-T}$, we can express the number of occupied beds on day $t$ as

$$
O_{t}\left(\mathbf{a}^{e}, \mathbf{a}_{t-L_{\max }}^{u}, \ldots, \mathbf{a}_{t}^{u}\right)=\sum_{i=1}^{n} \sum_{k=1}^{K_{i}} \sum_{T=0}^{L_{\max }^{i}}\left(a_{i}^{e}+a_{i, t-T}^{u}\right) q_{i k} I_{i, k, t-T}=\sum_{i=1}^{n} \sum_{k=1}^{K_{i}} q_{i k} N_{i, k, t},
$$


where

$$
N_{i, k, t}=\sum_{T=0}^{L_{\max }^{i}}\left(a_{i}^{e}+a_{i, t-T}^{u}\right) I_{i, k, t-T}
$$

with $I_{i, k, t-T}$ independent Bernoulli random variables. Note that, similar to $I_{i, k_{1}, t-T}$ and $I_{i, k_{2}, t-T}$, $N_{i, k_{1}, t}$ and $N_{i, k_{2}, t}$ are i.i.d. random variables for any pair of $k_{1}, k_{2} \in\left\{1,2, \ldots, K_{i}\right\}$ with $k_{1} \neq k_{2}$. Moreover, $I_{i, k, t-T}$ and $a_{i, t-T}$ are also independent. Then, the expectation and the variance of $N_{i, k, t}$ are given by

$$
E\left[N_{i, k, t}\right]=\sum_{T=0}^{L_{\max }^{i}}\left(a_{i}^{e}+a_{i, t-T}^{u}\right) E\left[I_{i, k, t-T}\right]=\sum_{T=0}^{L_{\max }^{i}}\left(a_{i}^{e}+a_{i, t-T}^{u}\right) p_{i, T}^{L}=a_{i}^{e} \mu^{N}+\sum_{T=0}^{L_{\max }^{i}} a_{i, t-T}^{u} p_{i, T}^{L},
$$

and

$$
\begin{aligned}
\operatorname{Var}\left[N_{i, k, t}\right] & =\sum_{T=0}^{L_{\max }^{i}} \operatorname{Var}\left[\left(a_{i}^{e}+a_{i, t-T}^{u}\right) I_{i, k, t-T}\right] \\
& =\sum_{T=0}^{L_{\max }^{i}}\left(a_{i}^{e}+a_{i, t-T}^{u}\right)^{2} \operatorname{Var}\left[I_{i, k, t-T}\right]=\sum_{T=0}^{L_{\max }^{i}}\left(a_{i}^{e}+a_{i, t-T}^{u}\right)^{2} p_{i, T}^{L}\left(1-p_{i, T}^{L}\right),
\end{aligned}
$$

where none of these quantities depends on $k$.

Similarly to the procedure duration case, we can set $X_{k}=q_{i k} N_{i, k, t}$. Then, defining

$$
\mathcal{N}_{i, t}=\sum_{k=1}^{K_{i}} q_{i k} N_{i, k, t}
$$

we can use Lyapunov Central Limit Theorem to express the limiting distribution of $\mathcal{N}_{i t}$ for any $t$ as the normal random variable with the mean of $E\left[N_{i, k, t}\right]$ and the standard deviation of $\sqrt{\operatorname{Var}\left[N_{i, k, t}\right] \sum_{k=1}^{K_{i}} q_{i k}^{2}}$. Then, the independence of $\mathcal{N}_{i_{1}, t}$ and $\mathcal{N}_{i_{2}, t}$ for any pair of procedure types $i_{1}, i_{2} \in\{1,2, \ldots, n\}$ with $i_{1} \neq i_{2}$ implies that:

$$
\begin{aligned}
& O_{t}\left(\mathbf{a}^{e}, \mathbf{a}_{t-L_{\max }^{u}}^{u}, \ldots, \mathbf{a}_{t}^{u}\right) \\
& \sim N\left(\sum_{i=1}^{n}\left(a_{i}^{e} \mu^{N}+\sum_{T=0}^{L_{\max }^{i}} a_{i, t-T}^{u} p_{i, T}^{L}\right),\right. \\
& \qquad \sqrt{\left.\sum_{i=1}^{n}\left(\sum_{T=0}^{L_{\max }^{i}}\left(a_{i}^{e}+a_{i, t-T}^{u}\right)^{2} p_{i, T}^{L}\left(1-p_{i, T}^{L}\right)\right)\left(\sum_{k=1}^{K_{i}} q_{i k}^{2}\right)\right)} \\
& =N\left(M^{N}\left(\mathbf{a}^{e}, \mathbf{a}_{t-L_{\max }^{u}}^{u}, \ldots, \mathbf{a}_{t}^{u}\right), \Sigma^{N}\left(\mathbf{a}^{e}, \mathbf{a}_{t-L_{\max }^{u}}^{u}, \ldots, \mathbf{a}_{t}^{u}\right)\right) .
\end{aligned}
$$




\section{Proposition A1}

Proposition A1. Suppose that procedures of type $i=1, \ldots, n$ in a subset $k=1, \ldots, K_{i}$, performed on day $t$, result in independent pairs of procedure and LOS durations $\left(S_{i, j_{1}, t}, L_{i, j_{1}, t}\right)$ and $\left(S_{i, j_{2}, t}, L_{i, j_{2}, t}\right)$, $j_{1}, j_{2} \in C_{i, k}$ and define

$$
\begin{aligned}
M^{S}\left(\mathbf{a}^{e}, \mathbf{a}_{t}^{u}\right) & =\sum_{i=1}^{n}\left(a_{i}^{e}+a_{i, t}^{u}\right) \mu_{i}^{S}, \\
\left(\Sigma^{S}\left(\mathbf{a}^{e}, \mathbf{a}_{t}^{u}\right)\right)^{2} & =\sum_{i=1}^{n}\left(a_{i}^{e}+a_{i, t}^{u}\right)\left(\sigma_{i}^{S}\right)^{2}, \\
M^{N}\left(\mathbf{a}^{e}, \mathbf{a}_{t-L_{\max }^{u}}^{u}, \ldots, \mathbf{a}_{t}^{u}\right) & =\sum_{i=1}^{n}\left(a_{i}^{e} \mu_{i}^{N}+\sum_{T=0}^{L_{\max }^{i}} a_{i, t-T}^{u} p_{i, T}^{L}\right), \\
\left(\Sigma^{N}\left(\mathbf{a}^{e}, \mathbf{a}_{t-L_{\max }^{u}}^{u}, \ldots, \mathbf{a}_{t}^{u}\right)\right)^{2} & =\sum_{i=1}^{n}\left(\sum_{T=0}^{L_{\max }^{i}}\left(a_{i}^{e}+a_{i, t-T}^{u}\right) p_{i, T}^{L}\left(1-p_{i, T}^{L}\right)\right) .
\end{aligned}
$$

Then, as $a_{i, t}=a_{i}^{e}+a_{i, t-T}^{u} \rightarrow \infty$,

$$
\frac{D_{t}\left(\mathbf{a}^{e}, \mathbf{a}_{t}^{u}\right)-M^{S}\left(\mathbf{a}^{e}, \mathbf{a}_{t}^{u}\right)}{\sum^{S}\left(\mathbf{a}^{e}, \mathbf{a}_{t}^{u}\right)} \stackrel{d}{\rightarrow} \mathcal{N}(0,1)
$$

and

$$
\frac{O_{t}\left(\mathbf{a}^{e}, \mathbf{a}_{t-L_{\max }^{u}}^{u}, \ldots, \mathbf{a}_{t}^{u}\right)-M^{N}\left(\mathbf{a}^{e}, \mathbf{a}_{t-L_{\max }^{u}}^{u}, \ldots, \mathbf{a}_{t}^{u}\right)}{\Sigma^{N}\left(\mathbf{a}^{e}, \mathbf{a}_{t-L_{\max }^{u}}^{u}, \ldots, \mathbf{a}_{t}^{u}\right)} \stackrel{d}{\rightarrow} \mathcal{N}(0,1) .
$$

Proof. In this case, all surgical procedures result in independent pairs of procedure and LOS durations, so that we do not need to differentiate them with respect to subset $k$. Accordingly, we will use the procedure and LOS durations $\left(S_{i, j, t}, L_{i, j, t}\right)$, which corresponds to the $j$-th procedure of type $i$ on day $t$. We also set $a_{i, t}=a_{i}^{e}+a_{i, t}^{u}$, so that $a_{i, t}$ denotes the total number of type $i$ procedures performed in day $t$.

Consider the total procedure duration due to type- $i$ procedures on day $t$, denoted by $D_{i, t}\left(a_{i, t}\right)$ :

$$
D_{i, t}\left(a_{i, t}\right)=\sum_{j=1}^{a_{i, t}} S_{i, j, t} .
$$

The expected value and variance of $D_{i, t}\left(a_{i, t}\right)$ are given by:

$$
\begin{aligned}
E\left[D_{i, t}\left(a_{i, t}\right)\right] & =E\left[\sum_{j=1}^{a_{i, t}} S_{i, j, t}\right]=a_{i, t} \mu_{i}^{S}, \\
\operatorname{Var}\left[D_{i, t}\left(a_{i, t}\right)\right] & =\operatorname{Var}\left[\sum_{j=1}^{a_{i, t}} S_{i, j, t}\right]=a_{i, t} \operatorname{Var}\left[S_{i, j, t}\right]=a_{i, t}\left(\sigma_{i}^{S}\right)^{2},
\end{aligned}
$$

since $S_{i, j, t}$ are independent and identical distributed (i.i.d.)for fixed $i$ and $t$. Moreover, due to i.i.d. nature of $S_{i, j, t}$, the central limit theorem ensures that $D_{i, t}\left(a_{i, t}\right)$ has an approximate normal distribution as $a_{i, t}$ goes to infinity. 
The total procedure duration procedures on day $t D_{t}\left(\mathbf{a}^{e}, \mathbf{a}_{t}^{u}\right)$ is the sum of $D_{i, t}\left(a_{i, t}\right)$ 's, which are all approximately normal as $a_{i, t} \rightarrow \infty$. Then $D_{t}\left(\mathbf{a}^{e}, \mathbf{a}_{t}^{u}\right)$ is also approximately normal with the following parameters:

$$
\begin{aligned}
E\left[D_{t}\left(\mathbf{a}^{e}, \mathbf{a}_{t}^{u}\right)\right] & =\sum_{i=1}^{n} a_{i, t} \mu_{i}^{S}, \\
\operatorname{Var}\left[D_{t}\left(\mathbf{a}^{e}, \mathbf{a}_{t}^{u}\right)\right] & =\sum_{i=1}^{n} a_{i, t}\left(\sigma_{i}^{S}\right)^{2},
\end{aligned}
$$

which proves Proposition A1 for $D_{t}\left(\mathbf{a}^{e}, \mathbf{a}_{t}^{u}\right)$.

To prove the proposition for $O_{t}\left(\mathbf{a}^{e}, \mathbf{a}_{t-L_{\max }}^{u}, \ldots, \mathbf{a}_{t}^{u}\right)$, we let $N_{i, t}^{T}$ denote the number of type- $i$ patients who underwent the operation on day $t-T$ and who are still at the hospital. Then $N_{i, t}^{T}$ is a Binomial random variable with parameters $\left(a_{i, t-T}, p_{i, T}^{L}\right)$ from the discussion in the proof of Proposition 1, so that $E\left[N_{i, t}^{T}\right]=a_{i, t-T} p_{i, T}^{L}$ and $\operatorname{Var}\left[N_{i, t}^{T}\right]=a_{i, t-T} p_{i, T}^{L}\left(1-p_{i, T}^{L}\right)$. As $a_{i, t-T} \rightarrow \infty$, $N_{i, t}^{T}$ has an approximate normal distribution due to the central limit theorem. On the other hand, $O_{t}\left(\mathbf{a}^{e}, \mathbf{a}_{t-L_{\max }^{u}}^{u}, \ldots, \mathbf{a}_{t}^{u}\right)$ can be written in terms of $N_{i, t}^{T}$ 's as follows:

$$
O_{t}\left(\mathbf{a}^{e}, \mathbf{a}_{t-L_{\max }}^{u}, \ldots, \mathbf{a}_{t}^{u}\right)=\sum_{i=1}^{n} \sum_{T=0}^{L_{\max }^{i}} N_{i, t}^{T} .
$$

Then $O_{t}\left(\mathbf{a}^{e}, \mathbf{a}_{t-L_{\max }}^{u}, \ldots, \mathbf{a}_{t}^{u}\right)$ is the sum of approximately normal random variables, so that it also has an approximately normal distribution with the following parameters:

$$
\begin{aligned}
E\left[O_{t}\left(\mathbf{a}^{e}, \mathbf{a}_{t-L_{\max }^{u}}^{u}, \ldots, \mathbf{a}_{t}^{u}\right)\right] & =\sum_{i=1}^{n}\left(\sum_{T=0}^{L_{\max }^{i}} a_{i, t-T} p_{i, T}^{L}\right), \\
\operatorname{Var}\left[O_{t}\left(\mathbf{a}^{e}, \mathbf{a}_{t-L_{\max }^{u}}^{u}, \ldots, \mathbf{a}_{t}^{u}\right)\right] & =\sum_{i=1}^{n}\left(\sum_{T=0}^{L_{\max }^{i}} a_{i, t} p_{i, T}^{L}\left(1-p_{i, T}^{L}\right)\right),
\end{aligned}
$$

which proves the second part of Proposition A1.

Note that similar to the assumption $K_{i} \rightarrow \infty$ required for the central-limit result of Proposition 1 , the analytical tractability under the "independence" assumption demands the limit $a_{i} \rightarrow \infty$. Thus, irrespective of the correlation structure one considers, an additional limiting assumption must be made in order to obtain a closed-form characterization of the impact of a particular portfolio of elective procedures on the use of hospital resources. The alternative correlation structure considered in Proposition A1, as expected, does not influence the first moments for the use of OR time or recovery beds: the expressions (A26) and (A28) are identical to their counterparts from Proposition 1, (20) and (22). The key difference between the expressions for the variance of the OR time and bed usage (A27) and (A29) under the "independence" assumption and their counterparts 
(21) and (23) under perfect correlation assumption: while the variance values (A27) and (A29) are proportional to the first powers of the daily procedure numbers, (21) and (23) are proportional to their second powers. This is not surprising: perfectly correlated durations are expected to produce, for the same numbers of daily procedures, higher variance in terms of the use of both resources. This, in turn, results in higher "overage" costs - and, consequently, may produce, for the same values of penalty $\operatorname{costs} \pi^{N}$ and $\pi^{S}$, smaller optimal numbers of daily elective procedures. Thus, ignoring the correlation structure described in the Assumption 5 may result in overutilization of hospital resources. With this in mind, we conduct our analysis below based on the more realistic Assumption 5 and the limiting distribution derived in Proposition 1.

\section{Proof of Lemma 1}

Letting $\mathbf{A}_{t}^{u}=\left(\mathbf{a}_{t-L_{\max }}^{u}, \ldots, \mathbf{a}_{t}^{u}\right)$ in this proof, we will denote the number of occupied beds on day $t$ by $O_{t}\left(\mathbf{a}^{e}, \mathbf{A}_{t}^{u}\right)$, which is distributed approximately normal with mean $M^{N}\left(\mathbf{a}^{e}, \mathbf{A}_{t}^{u}\right)$ and standard deviation $\Sigma^{N}\left(\mathbf{a}^{e}, \mathbf{A}_{t}^{u}\right)$ by Proposition 1.

To compute the expected penalty for exceeding the bed constraint, we need to derive an expression for the expected "overflow" in the number of occupied beds:

$$
\begin{aligned}
& E\left[\left(O_{t}\left(\mathbf{a}^{e}, \mathbf{A}_{t}^{u}\right)-c\right)^{+}\right] \\
& =\int_{l_{1}}^{h_{1}} \ldots \int_{l_{n}}^{h_{n}} d \boldsymbol{\xi}_{t-L_{\max }} f^{u}\left(\boldsymbol{\xi}_{t-L_{\max }}\right) \ldots \int_{l_{1}}^{h_{1}} \ldots \int_{l_{n}}^{h_{n}} d \boldsymbol{\xi}_{t} f^{u}\left(\boldsymbol{\xi}_{t}\right) \int_{c}^{+\infty} d x \frac{e^{-\frac{1}{2}\left(\frac{x-M^{N}\left(\mathbf{a}^{e}, \mathbf{\Xi}_{t}\right)}{\Sigma^{N}\left(\mathbf{a}^{e}, \mathbf{\Xi}_{t}\right)}\right)^{2}}}{\sqrt{2 \pi} \Sigma^{N}\left(\mathbf{a}^{e}, \mathbf{\Xi}_{t}\right)}(x-c) .
\end{aligned}
$$

Now we set:

$$
\int_{\boldsymbol{\Xi}^{u}} d \boldsymbol{\Xi}_{t} F^{u}\left(\boldsymbol{\Xi}_{t}\right)=\left(\int_{l_{1}}^{h_{1}} \ldots \int_{l_{n}}^{h_{n}}\right) \cdots\left(\int_{l_{1}}^{h_{1}} \ldots \int_{l_{n}}^{h_{n}}\right) d \boldsymbol{\xi}_{t-L_{\max }} \cdots d \boldsymbol{\xi}_{t}\left(f^{u}\left(\boldsymbol{\xi}_{t-L_{\max }}\right) \cdots f^{u}\left(\boldsymbol{\xi}_{t}\right)\right) .
$$

with

$$
\begin{aligned}
\boldsymbol{\Xi}^{u} & =\left(\left[l_{1}, h_{1}\right] \times \cdots \times\left[l_{n}, h_{n}\right]\right) \times \cdots \times\left(\left[l_{1}, h_{1}\right] \times \cdots \times\left[l_{n}, h_{n}\right]\right), \\
\boldsymbol{\Xi}_{t} & =\left(\boldsymbol{\xi}_{t-L_{\max }}, \cdots, \boldsymbol{\xi}_{t}\right), \\
d \boldsymbol{\Xi}_{t} & =d \boldsymbol{\xi}_{t-L_{\max }} \cdots d \boldsymbol{\xi}_{t}, \\
F^{u}\left(\boldsymbol{\Xi}_{t}\right) & =\prod_{T=0}^{L_{\max }} f^{u}\left(\boldsymbol{\xi}_{t-T}\right) .
\end{aligned}
$$

Then we have the following expression:

$$
E\left[\left(O_{t}\left(\mathbf{a}^{e}, \mathbf{A}_{t}^{u}\right)-c\right)^{+}\right]
$$




$$
\begin{aligned}
& =\int_{\boldsymbol{\Xi}^{u}} d \boldsymbol{\Xi}_{t} F^{u}\left(\boldsymbol{\Xi}_{t}\right) \frac{1}{\sqrt{2 \pi}} \int_{\frac{c-M^{N}\left(\mathbf{a}^{e}, \mathbf{\Xi}_{t}\right)}{\Sigma^{N}\left(\mathbf{a}^{e}, \mathbf{\Xi}_{t}\right)}}^{+\infty} d v e^{-\frac{1}{2} v^{2}}\left(v \Sigma^{N}\left(\mathbf{a}^{e}, \boldsymbol{\Xi}_{t}\right)+M^{N}\left(\mathbf{a}^{e}, \boldsymbol{\Xi}_{t}\right)-c\right) \\
& =\int_{\boldsymbol{\Xi}^{u}} d \boldsymbol{\Xi}_{t} F^{u}\left(\boldsymbol{\Xi}_{t}\right) \frac{1}{\sqrt{2 \pi}} \times \\
& \left(\Sigma^{N}\left(\mathbf{a}^{e}, \boldsymbol{\Xi}_{t}\right) e^{-\frac{1}{2}\left(\frac{c-M^{N}\left(\mathbf{a}^{e}, \boldsymbol{\Xi}_{t}\right)}{\Sigma^{N}\left(\mathbf{a}^{e}, \boldsymbol{\Xi}_{t}\right)}\right)^{2}}+\left(M^{N}\left(\mathbf{a}^{e}, \boldsymbol{\Xi}_{t}\right)-c\right) \sqrt{\frac{\pi}{2}} \operatorname{erfc}\left(\frac{c-M^{N}\left(\mathbf{a}^{e}, \boldsymbol{\Xi}_{t}\right)}{\sqrt{2} \Sigma^{N}\left(\mathbf{a}^{e}, \boldsymbol{\Xi}_{t}\right)}\right)\right) \\
& =\int_{\boldsymbol{\Xi}^{u}} d \boldsymbol{\Xi}_{t} F^{u}\left(\boldsymbol{\Xi}_{t}\right) \Sigma^{N}\left(\mathbf{a}^{e}, \boldsymbol{\Xi}_{t}\right) \times \\
& \left(\frac{1}{\sqrt{2 \pi}} e^{-\frac{1}{2}\left(\frac{c-M^{N}\left(\mathbf{a}^{e}, \boldsymbol{\Xi}_{t}\right)}{\Sigma^{N}\left(\mathbf{a}^{e}, \boldsymbol{\Xi}_{t}\right)}\right)^{2}}-\left(\frac{c-M^{N}\left(\mathbf{a}^{e}, \boldsymbol{\Xi}_{t}\right)}{2 \Sigma^{N}\left(\mathbf{a}^{e}, \boldsymbol{\Xi}_{t}\right)}\right) \operatorname{erfc}\left(\frac{c-M^{N}\left(\mathbf{a}^{e}, \boldsymbol{\Xi}_{t}\right)}{\sqrt{2} \Sigma^{N}\left(\mathbf{a}^{e}, \boldsymbol{\Xi}_{t}\right)}\right)\right)
\end{aligned}
$$

In a similar fashion,

$$
\begin{aligned}
& E\left[\left(D_{t}\left(\mathbf{a}^{e}, \mathbf{a}_{t}^{u}\right)-\tau\right)^{+}\right] \\
= & \int_{l_{1}}^{h_{1}} \ldots \int_{l_{n}}^{h_{n}} d \boldsymbol{\xi}_{t} f^{u}\left(\boldsymbol{\xi}_{t}\right)\left(\sqrt{\sum_{i=1}^{n}\left(a_{i}^{e}+\xi_{i, t}\right)^{2}\left(\bar{\sigma}_{i}^{S}\right)^{2}}\right)\left(\frac{1}{\sqrt{2 \pi}} e^{-\frac{1}{2}\left(\frac{\tau-\sum_{i=1}^{n}\left(a_{i}^{e}+\xi_{i, t}\right) \mu_{i}^{S}}{\sqrt{\sum_{i=1}^{n}\left(a_{i}^{e}+\xi_{i, t}\right)^{2}\left(\bar{\sigma}_{i}^{S}\right)^{2}}}\right)^{2}}\right. \\
& \left.-\frac{1}{2}\left(\frac{\tau-\sum_{i=1}^{n}\left(a_{i}^{e}+\xi_{i, t}\right) \mu_{i}^{S}}{\sqrt{\sum_{i=1}^{n}\left(a_{i}^{e}+\xi_{i, t}\right)^{2}\left(\bar{\sigma}_{i}^{S}\right)^{2}}}\right) \operatorname{erfc}\left(\frac{1}{\sqrt{2}} \frac{\tau-\sum_{i=1}^{n}\left(a_{i}^{e}+\xi_{i, t}\right) \mu_{i}^{S}}{\left(\sqrt{\sum_{i=1}^{n}\left(a_{i}^{e}+\xi_{i, t}\right)^{2}\left(\bar{\sigma}_{i}^{S}\right)^{2}}\right)}\right)\right)
\end{aligned}
$$

where

$$
\left(\bar{\sigma}_{i}^{S}\right)^{2}=\left(\sigma_{i}^{S}\right)^{2} \sum_{k=1}^{K_{i}} q_{i k}^{2} .
$$

Substituting (A46) and (A47) into (19), we get (27).

\section{Proof of Proposition 2}

a) When the number of elective procedures is set at $a^{e}$, the daily profit function $\Pi\left(a^{e}\right)$ becomes

$$
\begin{aligned}
\Pi\left(a^{e}\right)=R a^{e}+R \int_{l}^{h} \xi f^{u}(\xi) d \xi \\
-\frac{\pi^{S} \bar{\sigma}^{S}}{\sqrt{2 \pi}}\left(\int_{l}^{h} d \xi f^{u}(\xi)\left(a^{e}+\xi\right)\left(e^{-\frac{1}{2}\left(\frac{\tau}{\left(a^{e}+\xi\right) \bar{\sigma}^{S}}-\rho^{S}\right)^{2}}-\left(\frac{\tau}{\left(a^{e}+\xi\right) \bar{\sigma}^{S}}-\rho^{S}\right) \int_{\frac{\tau}{\left(a^{e}+\xi\right) \bar{\sigma}^{S}}-\rho^{S}}^{+\infty} e^{-\frac{1}{2} v^{2}} d v\right)\right) \\
-\frac{\pi^{N}}{\sqrt{2 \pi}} \int_{\Xi^{u}} d \boldsymbol{\Xi}_{t} F^{u}\left(\boldsymbol{\Xi}_{t}\right) \Sigma^{N}\left(a^{e}, \boldsymbol{\Xi}_{t}\right)\left(e^{-\frac{1}{2}\left(\frac{c-M^{N}\left(a^{e}, \mathbf{\Xi}_{t}\right)}{\Sigma^{N}\left(a^{e}, \mathbf{\Xi}_{t}\right)}\right)^{2}}-\left(\frac{c-M^{N}\left(a^{e}, \mathbf{\Xi}_{t}\right)}{\Sigma^{N}\left(a^{e}, \mathbf{\Xi}_{t}\right)}\right) \int_{\frac{c-\mu^{N}\left(a^{e}, \cdot\right)}{\Sigma^{N}\left(a^{e}, \mathbf{\Xi}_{t}\right)}}^{+\infty} e^{-\frac{1}{2} v^{2}} d v\right) .
\end{aligned}
$$


The second derivative of the first two terms are 0 . So, it is sufficient to show that the remaining two terms have negative second derivatives. Setting $g^{S}\left(a^{e}, \xi\right)$ and $g^{N}\left(a^{e}, \boldsymbol{\Xi}_{t}\right)$ as:

$$
\begin{aligned}
& g^{S}\left(a^{e}, \xi\right)=\left(a^{e}+\xi\right)\left(e^{-\frac{1}{2}\left(\frac{\tau}{\left(a^{e}+\xi\right) \bar{\sigma}^{S}}-\rho^{S}\right)^{2}}-\left(\frac{\tau}{\left(a^{e}+\xi\right) \bar{\sigma}^{S}}-\rho^{S}\right) \int_{\frac{\tau}{\left(a^{e}+\xi\right) \bar{\sigma}^{S}}-\rho^{S}}^{+\infty} e^{-\frac{1}{2} v^{2}} d v\right), \\
& g^{N}\left(a^{e}, \boldsymbol{\Xi}_{t}\right)=\Sigma^{N}\left(a^{e}, \boldsymbol{\Xi}_{t}\right)\left(e^{-\frac{1}{2}\left(\frac{c-M^{N}\left(a^{e}, \boldsymbol{\Xi}_{t}\right)}{\Sigma^{N}\left(a^{e}, \mathbf{\Xi}_{t}\right)}\right)^{2}}-\left(\frac{c-M^{N}\left(a^{e}, \boldsymbol{\Xi}_{t}\right)}{\Sigma^{N}\left(a^{e}, \boldsymbol{\Xi}_{t}\right)}\right) \int_{\frac{c-M^{N}\left(a^{e}, \mathbf{\Xi}_{t}\right)}{\Sigma^{N}\left(a^{e}, \mathbf{\Xi}_{t}\right)}}^{+\infty} e^{-\frac{1}{2} v^{2}} d v\right),
\end{aligned}
$$

$\Pi\left(a^{e}\right)$ can be written as follows:

$$
\Pi\left(a^{e}\right)=R\left(a^{e}+\bar{\xi}\right)-\frac{\pi^{S} \bar{\sigma}^{S}}{\sqrt{2 \pi}} \int_{l}^{h} d \xi f^{u}(\xi) g^{S}\left(a^{e}, \xi\right)-\frac{\pi^{N}}{\sqrt{2 \pi}} \int_{\Xi^{u}} d \boldsymbol{\Xi}_{t} F^{u}\left(\boldsymbol{\Xi}_{t}\right) g^{N}\left(a^{e}, \boldsymbol{\Xi}_{t}\right) .
$$

The first and second derivatives $g^{S}$ with respect to $a^{e}$ are given by:

$$
\begin{aligned}
\frac{\partial g^{S}}{\partial a^{e}} & =e^{-\frac{1}{2}\left(\frac{\tau}{\left(a^{e}+\xi\right) \bar{\sigma}^{S}}-\rho^{S}\right)^{2}}+\rho^{S} \int_{\frac{\tau}{\left(a^{e}+\xi\right) \bar{\sigma}^{S}}-\rho^{S}}^{+\infty} e^{-\frac{1}{2} v^{2}} d v, \\
\frac{\partial^{2} g^{S}}{\partial\left(a^{e}\right)^{2}} & =\frac{\tau}{\left(a^{e}+\xi\right) \bar{\sigma}^{S}} e^{-\frac{1}{2}\left(\frac{\tau}{\left(a^{e}+\xi\right) \bar{\sigma}^{S}}-\rho^{S}\right)^{2}} \frac{\tau}{\left(a^{e}+\xi\right)^{2} \bar{\sigma}^{S}}>0,
\end{aligned}
$$

proving the second derivative of the second term is negative.

Now we write $g^{N}$ in terms of function $\alpha\left(a^{e}, \mathbf{\Xi}_{t}\right)$ :

$$
g^{N}\left(a^{e}, \boldsymbol{\Xi}_{t}\right)=\Sigma^{N}\left(a^{e}, \boldsymbol{\Xi}_{t}\right)\left(e^{-\frac{1}{2} \alpha\left(a^{e}, \boldsymbol{\Xi}_{t}\right)^{2}}-\alpha\left(a^{e}, \boldsymbol{\Xi}_{t}\right) \int_{\alpha\left(a^{e}, \boldsymbol{\Xi}_{t}\right)}^{+\infty} e^{-\frac{1}{2} v^{2}} d v\right)
$$

where

$$
\alpha\left(a^{e}, \boldsymbol{\Xi}_{t}\right)=\frac{c-M^{N}\left(a^{e}, \boldsymbol{\Xi}_{t}\right)}{\Sigma^{N}\left(a^{e}, \mathbf{\Xi}_{t}\right)} .
$$

The following notation will be useful to simplify the expressions:

$$
\begin{aligned}
A^{(m)}\left(x, \boldsymbol{\Xi}_{t}\right) & =\sum_{T=0}^{L_{\max }}\left(x+\xi_{t-T}\right)^{m} p_{T}^{L}\left(1-p_{T}^{L}\right) \quad \text { for } m=0,1,2 . \\
Q & =\sum_{k=1}^{K} q_{k}^{2} .
\end{aligned}
$$

Note that $A^{(0)}\left(x, \boldsymbol{\Xi}_{t}\right)=\sum_{T=0}^{L_{\max }} p_{T}^{L}\left(1-p_{T}^{L}\right)$ for all $x$ and $\boldsymbol{\Xi}_{t}$, so we denote it by $A^{(0)}$. Moreover, $\Sigma^{N}\left(a^{e}, \boldsymbol{\Xi}_{t}\right)=\sqrt{Q A^{(2)}\left(a^{e}, \boldsymbol{\Xi}_{t}\right)}, \partial A^{(2)} / \partial x=2 A^{(1)}$, and $\partial A^{(1)} / \partial x=A^{(0)}$. 
Now we can present the derivative of the functions in (A55):

$$
\begin{aligned}
\frac{\partial M^{N}}{\partial a^{e}} & =\mu^{N}, \\
\frac{\partial \Sigma^{N}}{\partial a^{e}} & =\frac{\sqrt{Q} A^{(1)}\left(a^{e}, \boldsymbol{\Xi}_{t}\right)}{\sqrt{A^{(2)}\left(a^{e}, \boldsymbol{\Xi}_{t}\right)}}, \\
\frac{\partial \alpha}{\partial a^{e}} & =\frac{-\mu^{N} \Sigma^{N}\left(a^{e}, \boldsymbol{\Xi}_{t}\right)-\left(c-M^{N}\left(a^{e}, \boldsymbol{\Xi}_{t}\right)\right) \frac{\sqrt{Q} A^{(1)}\left(a^{e}, \boldsymbol{\Xi}_{t}\right)}{\sqrt{A^{(2)}\left(a^{e}, \boldsymbol{\Xi}_{t}\right)}}}{\left(\Sigma^{N}\left(a^{e}, \boldsymbol{\Xi}_{t}\right)\right)^{2}} \\
& =\frac{-\mu^{N} \sqrt{Q A^{(2)}\left(a^{e}, \boldsymbol{\Xi}_{t}\right)}-\left(c-M^{N}\left(a^{e}, \boldsymbol{\Xi}_{t}\right)\right) \frac{\sqrt{Q} A^{(1)}\left(a^{e}, \boldsymbol{\Xi}_{t}\right)}{\sqrt{A^{(2)}\left(a^{e}, \boldsymbol{\Xi}_{t}\right)}}}{Q A^{(2)}\left(a^{e}, \boldsymbol{\Xi}_{t}\right)} \\
= & -\frac{\mu^{N}}{\sqrt{Q A^{(2)}\left(a^{e}, \boldsymbol{\Xi}_{t}\right)}}-\alpha\left(a^{e}, \boldsymbol{\Xi}_{t}\right) \frac{A^{(1)}\left(a^{e}, \boldsymbol{\Xi}_{t}\right)}{A^{(2)}\left(a^{e}, \mathbf{\Xi}_{t}\right)},
\end{aligned}
$$

Then the first and second derivatives of $g^{N}$ are as follows:

$$
\begin{aligned}
\frac{\partial g^{N}}{\partial a^{e}} & =\frac{\sqrt{Q} A^{(1)}\left(a^{e}, \boldsymbol{\Xi}_{t}\right)}{\sqrt{A^{(2)}\left(a^{e}, \boldsymbol{\Xi}_{t}\right)}}\left(e^{-\frac{1}{2}\left(\alpha\left(a^{e}, \boldsymbol{\Xi}_{t}\right)\right)^{2}}-\alpha\left(a^{e}, \boldsymbol{\Xi}_{t}\right) \int_{\alpha\left(a^{e}, \boldsymbol{\Xi}_{t}\right)}^{+\infty} e^{-\frac{1}{2} v^{2}} d v\right) \\
& +\sqrt{Q A^{(2)}\left(a^{e}, \boldsymbol{\Xi}_{t}\right)}\left(\int_{\alpha\left(a^{e}, \boldsymbol{\Xi}_{t}\right)}^{+\infty} e^{-\frac{1}{2} v^{2}} d v\right)\left(\frac{\mu^{N}}{\sqrt{Q A^{(2)}\left(a^{e}, \boldsymbol{\Xi}_{t}\right)}}+\alpha\left(a^{e}, \boldsymbol{\Xi}_{t}\right) \frac{A^{(1)}\left(a^{e}, \boldsymbol{\Xi}_{t}\right)}{A^{(2)}\left(a^{e}, \boldsymbol{\Xi}_{t}\right)}\right) \\
& =\frac{\sqrt{Q} A^{(1)}\left(a^{e}, \boldsymbol{\Xi}_{t}\right)}{\sqrt{A^{(2)}\left(a^{e}, \boldsymbol{\Xi}_{t}\right)}} e^{-\frac{1}{2}\left(\alpha\left(a^{e}, \boldsymbol{\Xi}_{t}\right)\right)^{2}}+\mu^{N} \int_{\alpha\left(a^{e}, \boldsymbol{\Xi}_{t}\right)}^{+\infty} e^{-\frac{1}{2} v^{2}} d v,
\end{aligned}
$$

and

$$
\begin{aligned}
\frac{\partial^{2} g^{N}}{\partial\left(a^{e}\right)^{2}}= & -\frac{\sqrt{Q} A^{(1)}\left(a^{e}, \boldsymbol{\Xi}_{t}\right)}{\sqrt{A^{(2)}\left(a^{e}, \boldsymbol{\Xi}_{t}\right)}} \frac{\partial \alpha}{\partial a^{e}} \alpha\left(a^{e}, \boldsymbol{\Xi}_{t}\right) e^{-\frac{1}{2}\left(\alpha\left(a^{e}, \boldsymbol{\Xi}_{t}\right)\right)^{2}} \\
& +\sqrt{Q} e^{-\frac{1}{2}\left(\alpha\left(a^{e}, \boldsymbol{\Xi}_{t}\right)\right)^{2}}\left(\frac{A^{(0)} A^{(2)}\left(a^{e}, \boldsymbol{\Xi}_{t}\right)-\left(A^{(1)}\left(a^{e}, \boldsymbol{\Xi}_{t}\right)\right)^{2}}{\left(A^{(2)}\left(a^{e}, \boldsymbol{\Xi}_{t}\right)\right)^{\frac{3}{2}}}\right)-\mu^{N} \frac{\partial \alpha}{\partial a^{e}} e^{-\frac{1}{2}\left(\alpha\left(a^{e}, \boldsymbol{\Xi}_{t}\right)\right)^{2}} \\
= & e^{-\frac{1}{2}\left(\alpha\left(a^{e}, \boldsymbol{\Xi}_{t}\right)\right)^{2}} \times \\
& \left(-\mu^{N} \frac{\partial \alpha}{\partial a^{e}}-\frac{\sqrt{Q} A^{(1)}\left(a^{e}, \boldsymbol{\Xi}_{t}\right)}{\sqrt{A^{(2)}\left(a^{e}, \boldsymbol{\Xi}_{t}\right)}} \frac{\partial \alpha}{\partial a^{e}} \alpha\left(a^{e}, \boldsymbol{\Xi}_{t}\right)+\sqrt{Q}\left(\frac{A^{(0)} A^{(2)}\left(a^{e}, \boldsymbol{\Xi}_{t}\right)-\left(A^{(1)}\left(a^{e}, \boldsymbol{\Xi}_{t}\right)\right)^{2}}{\left(A^{(2)}\left(a^{e}, \boldsymbol{\Xi}_{t}\right)\right)^{\frac{3}{2}}}\right)\right) .
\end{aligned}
$$

This quantity is positive only if the following inequality is satisfied:

$$
-\mu^{N} \frac{\partial \alpha}{\partial a^{e}}-\frac{\sqrt{Q} A^{(1)}\left(a^{e}, \boldsymbol{\Xi}_{t}\right)}{\sqrt{A^{(2)}\left(a^{e}, \boldsymbol{\Xi}_{t}\right)}} \frac{\partial \alpha}{\partial a^{e}} \alpha\left(a^{e}, \boldsymbol{\Xi}_{t}\right)+\sqrt{Q}\left(\frac{A^{(0)} A^{(2)}\left(a^{e}, \boldsymbol{\Xi}_{t}\right)-\left(A^{(1)}\left(a^{e}, \boldsymbol{\Xi}_{t}\right)\right)^{2}}{\left(A^{(2)}\left(a^{e}, \boldsymbol{\Xi}_{t}\right)\right)^{\frac{3}{2}}}\right) \geq 0 .
$$

Plugging in the previous expressions, we have:

$$
\frac{\left(\mu^{N}\right)^{2}}{\sqrt{Q A^{(2)}\left(a^{e}, \boldsymbol{\Xi}_{t}\right)}}+\frac{\mu^{N} A^{(1)}\left(a^{e}, \boldsymbol{\Xi}_{t}\right)}{A^{(2)}\left(a^{e}, \boldsymbol{\Xi}_{t}\right)} \alpha\left(a^{e}, \boldsymbol{\Xi}_{t}\right)+\frac{\mu^{N} A^{(1)}\left(a^{e}, \boldsymbol{\Xi}_{t}\right)}{A^{(2)}\left(a^{e}, \boldsymbol{\Xi}_{t}\right)} \alpha\left(a^{e}, \boldsymbol{\Xi}_{t}\right)
$$




$$
+\sqrt{Q} \frac{\left(A^{(1)}\left(a^{e}, \boldsymbol{\Xi}_{t}\right)\right)^{2}}{\left(A^{(2)}\left(a^{e}, \boldsymbol{\Xi}_{t}\right)\right)^{\frac{3}{2}}} \alpha\left(a^{e}, \boldsymbol{\Xi}_{t}\right)^{2}+\sqrt{Q} \frac{A^{(0)} A^{(2)}\left(a^{e}, \boldsymbol{\Xi}_{t}\right)-\left(A^{(1)}\left(a^{e}, \boldsymbol{\Xi}_{t}\right)\right)^{2}}{\left(A^{(2)}\left(a^{e}, \boldsymbol{\Xi}_{t}\right)\right)^{\frac{3}{2}}} \geq 0 .
$$

The first four terms can be written in terms of a perfect square:

$$
\begin{aligned}
& \frac{\left(\mu^{N}\right)^{2}}{\sqrt{Q A^{(2)}\left(a^{e}, \boldsymbol{\Xi}_{t}\right)}}+2 \frac{\mu^{N} A^{(1)}\left(a^{e}, \boldsymbol{\Xi}_{t}\right)}{A^{(2)}\left(a^{e}, \boldsymbol{\Xi}_{t}\right)} \alpha\left(a^{e}, \boldsymbol{\Xi}_{t}\right)+\sqrt{Q} \frac{\left(A^{(1)}\left(a^{e}, \boldsymbol{\Xi}_{t}\right)\right)^{2}}{\left(A^{(2)}\left(a^{e}, \boldsymbol{\Xi}_{t}\right)\right)^{\frac{3}{2}}} \alpha\left(a^{e}, \boldsymbol{\Xi}_{t}\right)^{2} \\
= & \frac{1}{\sqrt{Q A^{(2)}\left(a^{e}, \boldsymbol{\Xi}_{t}\right)}}\left(\left(\mu^{N}\right)^{2}+2 \frac{\sqrt{Q} \mu^{N} A^{(1)}\left(a^{e}, \boldsymbol{\Xi}_{t}\right)}{\sqrt{A^{(2)}\left(a^{e}, \boldsymbol{\Xi}_{t}\right)}} \alpha\left(a^{e}, \boldsymbol{\Xi}_{t}\right)+Q \frac{\left(A^{(1)}\left(a^{e}, \boldsymbol{\Xi}_{t}\right)\right)^{2}}{A^{(2)}\left(a^{e}, \boldsymbol{\Xi}_{t}\right)} \alpha\left(a^{e}, \boldsymbol{\Xi}_{t}\right)^{2}\right) \\
= & \frac{1}{\sqrt{Q A^{(2)}\left(a^{e}, \boldsymbol{\Xi}_{t}\right)}}\left(\mu^{N}+\frac{\sqrt{Q} A^{(1)}\left(a^{e}, \boldsymbol{\Xi}_{t}\right)}{\sqrt{A^{(2)}\left(a^{e}, \boldsymbol{\Xi}_{t}\right)}} \alpha\left(a^{e}, \boldsymbol{\Xi}_{t}\right)\right)^{2} \geq 0 .
\end{aligned}
$$

Now consider the numerator of the last term. By plugging in the definitions of $A^{(m)}$ for $m=0,1,2$, we need to show that:

$$
\begin{aligned}
& \sum_{T_{1}=0}^{L_{\max }} \sum_{T_{2}=0}^{L_{\max }}\left(\left(a^{e}+\xi_{t-T_{1}}\right)^{2} p_{T_{1}}^{L}\left(1-p_{T_{1}}^{L}\right)\right)\left(p_{T_{2}}^{L}\left(1-p_{T_{2}}^{L}\right)\right) \\
- & \sum_{T_{1}=0}^{L_{\max }} \sum_{T_{2}=0}^{L_{\max }}\left(\left(a^{e}+\xi_{t-T_{1}}\right) p_{T_{1}}^{L}\left(1-p_{T_{1}}^{L}\right)\right)\left(\left(a^{e}+\xi_{t-T_{2}}\right) p_{T_{2}}^{L}\left(1-p_{T_{2}}^{L}\right)\right) \geq 0 .
\end{aligned}
$$

Now we define function $h(T)$ as:

$$
h(T)=\frac{p_{T}^{L}\left(1-p_{T}^{L}\right)}{\sum_{T=0}^{L_{\max }}\left(p_{T}^{L}\left(1-p_{T}^{L}\right)\right)} .
$$

Note that $h(T)$ can be interpreted as a probability mass function of a random variable $X$, where $X$ will have a value of $a^{e}+\xi_{t-T}$ with probability $h(T)$. Then dividing all terms in inequality (A67) by $\left(\sum_{T=0}^{L_{\max }}\left(p_{T}^{L}\left(1-p_{T}^{L}\right)\right)\right)^{2}$, we have:

$$
\sum_{T_{1}=0}^{L_{\max }} \sum_{T_{2}=0}^{L_{\max }}\left(\left(a^{e}+\xi_{t-T_{1}}\right)^{2} h\left(T_{1}\right) h\left(T_{2}\right)\right)-\sum_{T_{1}=0}^{L_{\max }} \sum_{T_{2}=0}^{L_{\max }}\left(\left(a^{e}+\xi_{t-T_{1}}\right) h\left(T_{1}\right)\right)\left(\left(a^{e}+\xi_{t-T_{2}}\right) h\left(T_{2}\right)\right) \geq 0,
$$

which can be written as follows in terms of random variable $X$ :

$$
E\left[X^{2}\right]-(E[X])^{2}=\operatorname{Var}[X] \geq 0 .
$$

Therefore, the last term is also always positive, proving the concavity of the objective function.

Note that the first derivative of the objective function with respect to $a^{e}$ is

$$
\begin{aligned}
& \frac{\partial \Pi}{\partial a^{e}}=R-\frac{\pi^{S} \bar{\sigma}^{S}}{\sqrt{2 \pi}}\left(\int_{l}^{h} d \xi f^{u}(\xi)\left(e^{-\frac{1}{2}\left(\frac{\tau}{\left(a^{e}+\xi\right) \bar{\sigma}^{S}}-\rho^{S}\right)^{2}}+\rho^{S} \int_{\frac{\tau}{\left(a^{e}+\xi\right) \bar{\sigma}^{S}}-\rho^{S}}^{+\infty} e^{-\frac{1}{2} v^{2}} d v\right)\right) \\
& -\frac{\pi^{N}}{\sqrt{2 \pi}} \int_{\boldsymbol{\Xi}^{u}} d \boldsymbol{\Xi}_{t} F^{u}\left(\boldsymbol{\Xi}_{t}\right)\left(\mu^{N} \int_{\alpha\left(a^{e}, \boldsymbol{\Xi}_{t}\right)}^{+\infty} e^{-\frac{1}{2} v^{2}} d v+\sqrt{Q} \frac{A^{(1)}\left(a^{e}, \boldsymbol{\Xi}_{t}\right)}{\sqrt{A^{(2)}\left(a^{e}, \mathbf{\Xi}_{t}\right)}} e^{-\frac{1}{2} \alpha\left(a^{e}, \boldsymbol{\Xi}_{t}\right)^{2}}\right) .
\end{aligned}
$$


b) Note that

$$
\begin{gathered}
\lim _{a^{e} \rightarrow 0} \frac{\partial \Pi}{\partial a^{e}}=R-\frac{\pi^{S} \bar{\sigma}^{S}}{\sqrt{2 \pi}}\left(\int_{l}^{h} d \xi f^{u}(\xi)\left(e^{-\frac{1}{2}\left(\frac{\tau}{\xi \bar{\sigma} S}-\rho^{S}\right)^{2}}+\rho^{S} \int_{\frac{\tau}{\xi \bar{\sigma} S}-\rho^{S}}^{+\infty} e^{-\frac{1}{2} v^{2}} d v\right)\right) \\
-\frac{\pi^{N}}{\sqrt{2 \pi}} \int_{\Xi^{u}} d \boldsymbol{\Xi}_{t} F^{u}\left(\boldsymbol{\Xi}_{t}\right)\left(\mu^{N} \int_{\alpha\left(0, \boldsymbol{\Xi}_{t}\right)}^{+\infty} e^{-\frac{1}{2} v^{2}} d v+\sqrt{Q} \frac{A^{(1)}\left(0, \boldsymbol{\Xi}_{t}\right)}{\sqrt{A^{(2)}\left(0, \mathbf{\Xi}_{t}\right)}} e^{-\frac{1}{2} \alpha\left(0, \boldsymbol{\Xi}_{t}\right)^{2}}\right) .
\end{gathered}
$$

Thus, for

$$
\begin{aligned}
& R \leq \frac{\pi^{N}}{\sqrt{2 \pi}} \int_{\Xi^{u}} d \boldsymbol{\Xi}_{t} F^{u}\left(\boldsymbol{\Xi}_{t}\right)\left(\mu^{N} \int_{\alpha\left(0, \boldsymbol{\Xi}_{t}\right)}^{+\infty} e^{-\frac{1}{2} v^{2}} d v+\sqrt{Q} \frac{A^{(1)}\left(0, \boldsymbol{\Xi}_{t}\right)}{\sqrt{A^{(2)}\left(0, \boldsymbol{\Xi}_{t}\right)}} e^{-\frac{1}{2} \alpha\left(0, \boldsymbol{\Xi}_{t}\right)^{2}}\right) \\
& +\frac{\pi^{S} \bar{\sigma}^{S}}{\sqrt{2 \pi}}\left(\int_{l}^{h} d \xi f^{u}(\xi)\left(e^{-\frac{1}{2}\left(\frac{\tau}{\xi \bar{\sigma} S}-\rho^{S}\right)^{2}}+\rho^{S} \int_{\frac{\tau}{\xi \bar{\sigma} S}-\rho^{S}}^{+\infty} e^{-\frac{1}{2} v^{2}} d v\right)\right)
\end{aligned}
$$

the optimal value of the number of elective procedures is $\hat{a}^{e}=0$.

c) Further,

$$
\begin{aligned}
& \lim _{a^{e} \rightarrow+\infty} \frac{\partial \Pi}{\partial a^{e}}= R-\frac{\pi^{N}}{\sqrt{2 \pi}} \int_{\Xi^{u}} d \boldsymbol{\Xi}_{t} F^{u}\left(\boldsymbol{\Xi}_{t}\right)\left(\mu^{N} \int_{-\frac{\mu^{N}}{\sqrt{Q A^{(0)}}}}^{+\infty} e^{-\frac{1}{2} v^{2}} d v+\sqrt{Q A^{(0)}} e^{-\frac{1}{2} \frac{\left(\mu^{N}\right)^{2}}{Q A^{(0)}}}\right) \\
&-\frac{\pi^{S} \bar{\sigma}^{S}}{\sqrt{2 \pi}}\left(\int_{l}^{h} d \xi f^{u}(\xi)\left(e^{-\frac{1}{2}\left(\rho^{S}\right)^{2}}+\sqrt{\frac{\pi}{2}} \rho^{S} \operatorname{erfc}\left(\frac{1}{\sqrt{2}}\left(-\rho^{S}\right)\right)\right)\right) \\
&= R-\frac{\pi^{N} \sqrt{Q A^{(0)}}}{\sqrt{2 \pi}}\left(\frac{\mu^{N}}{\sqrt{Q A^{(0)}}} \int_{\mu^{N}}^{+\infty} e^{-\frac{1}{2} v^{2}} d v+e^{-\frac{1}{2} \frac{\left(\mu^{N}\right)^{2}}{Q A^{(0)}}}\right) \\
&-\frac{\pi^{S} \bar{\sigma}^{S}}{\sqrt{2 \pi}}\left(e^{-\frac{1}{2}\left(\rho^{S}\right)^{2}}+\sqrt{\frac{\pi}{2} \rho^{S} \operatorname{erfc}}\left(\frac{1}{\sqrt{2}}\left(-\rho^{S}\right)\right)\right) \\
&= R-\frac{\pi^{N} \sqrt{Q A^{(0)}}}{\sqrt{2 \pi}}\left(e^{-\frac{1}{2}\left(\frac{\mu^{N}}{\sqrt{Q A^{(0)}}}\right)^{2}}+\sqrt{\frac{\pi}{2}} \frac{\mu^{N}}{\sqrt{Q A^{(0)}}} \operatorname{erfc}\left(\frac{1}{\sqrt{2}}\left(-\frac{\mu^{N}}{\sqrt{Q A^{(0)}}}\right)\right)\right) \\
&-\frac{\pi^{S} \bar{\sigma}^{S}}{\sqrt{2 \pi}}\left(e^{-\frac{1}{2}\left(\rho^{S}\right)^{2}}+\sqrt{\frac{\pi}{2} \rho^{S} \operatorname{erfc}}\left(\frac{1}{\sqrt{2}}\left(-\rho^{S}\right)\right)\right) . \\
&(\mathrm{A} 7
\end{aligned}
$$

Thus, for

$$
R>\frac{\pi^{N} \sqrt{Q A^{(0)}}}{\sqrt{2 \pi}}\left(e^{-\frac{1}{2}\left(\frac{\mu^{N}}{\sqrt{Q A^{(0)}}}\right)^{2}}+\sqrt{\frac{\pi}{2}} \frac{\mu^{N}}{\sqrt{Q A^{(0)}}} \operatorname{erfc}\left(\frac{1}{\sqrt{2}}\left(-\frac{\mu^{N}}{\sqrt{Q A^{(0)}}}\right)\right)\right)
$$




$$
+\frac{\pi^{S} \bar{\sigma}^{S}}{\sqrt{2 \pi}}\left(e^{-\frac{1}{2}\left(\rho^{S}\right)^{2}}+\sqrt{\frac{\pi}{2}} \rho^{S} \operatorname{erfc}\left(\frac{1}{\sqrt{2}}\left(-\rho^{S}\right)\right)\right)
$$

it is optimal to perform as many surgical procedures as possible, so that $\hat{a}^{e}=U$. Note that $\bar{\sigma}^{N}=$ $\sqrt{Q A^{(0)}}$.

d) First consider the problem without demand constraints, and denote the optimal number of daily elective procedures in this setting by $\hat{a}_{\infty}^{e}$. Then for

$$
\begin{aligned}
& \frac{\pi^{N}}{\sqrt{2 \pi}} \int_{\boldsymbol{\Xi}^{u}} d \boldsymbol{\Xi}_{t} F^{u}\left(\boldsymbol{\Xi}_{t}\right)\left(\mu^{N} \int_{\alpha\left(0, \Xi_{t}\right)}^{+\infty} e^{-\frac{1}{2} v^{2}} d v+\sqrt{Q} \frac{A^{(1)}\left(0, \boldsymbol{\Xi}_{t}\right)}{\sqrt{A^{(2)}\left(0, \boldsymbol{\Xi}_{t}\right)}} e^{-\frac{1}{2} \alpha\left(0, \boldsymbol{\Xi}_{t}\right)^{2}}\right) \\
+ & \frac{\pi^{S} \bar{\sigma}^{S}}{\sqrt{2 \pi}}\left(\int_{l}^{h} d \xi f^{u}(\xi)\left(e^{-\frac{1}{2}\left(\frac{\tau}{\xi \bar{\sigma} S^{S}}-\rho^{S}\right)^{2}}+\rho^{S} \int_{\frac{\tau}{\xi \bar{\sigma}^{S}}-\rho^{S}}^{+\infty} e^{-\frac{1}{2} v^{2}} d v\right)\right) \\
\leq & R \leq \frac{\pi^{N} \Sigma^{N}\left(a^{e}, \boldsymbol{\Xi}_{t}\right) \sqrt{Q A^{(0)}}}{\sqrt{2 \pi}}\left(e^{-\frac{1}{2}\left(\frac{\mu^{N}}{\sqrt{Q A^{(0)}}}\right)^{2}}+\sqrt{\frac{\pi}{2}} \frac{\mu^{N}}{\sqrt{Q A^{(0)}}} \operatorname{erfc}\left(\frac{1}{\sqrt{2}}\left(-\frac{\mu^{N}}{\sqrt{Q A^{(0)}}}\right)\right)\right) \\
& +\frac{\pi^{S} \bar{\sigma}^{S}}{\sqrt{2 \pi}}\left(e^{-\frac{1}{2}\left(\rho^{S}\right)^{2}}+\sqrt{\frac{\pi}{2}} \rho^{S} \operatorname{erfc}\left(\frac{1}{\sqrt{2}}\left(-\rho^{S}\right)\right)\right)
\end{aligned}
$$

$\hat{a}_{\infty}^{e}$ is given by a unique solution to

$$
\begin{aligned}
R= & \frac{\pi^{N}}{\sqrt{2 \pi}} \int_{\boldsymbol{\Xi}^{u}} d \boldsymbol{\Xi}_{t} F^{u}\left(\boldsymbol{\Xi}_{t}\right)\left(\mu^{N} \int_{\alpha\left(a^{e}, \boldsymbol{\Xi}_{t}\right)}^{+\infty} e^{-\frac{1}{2} v^{2}} d v+\sqrt{Q} \frac{A^{(1)}\left(a^{e}, \boldsymbol{\Xi}_{t}\right)}{\sqrt{A^{(2)}\left(a^{e}, \mathbf{\Xi}_{t}\right)}} e^{-\frac{1}{2} \alpha\left(a^{e}, \boldsymbol{\Xi}_{t}\right)^{2}}\right) \\
& +\frac{\pi^{S} \bar{\sigma}^{S}}{\sqrt{2 \pi}}\left(\int_{l}^{h} d \xi f^{u}(\xi)\left(e^{-\frac{1}{2}\left(\frac{\tau}{\left(a^{e}+\xi\right) \bar{\sigma}^{S}}-\rho^{S}\right)^{2}}+\rho^{S} \int_{\frac{\tau}{\left(a^{e}+\xi\right) \bar{\sigma}^{S}}-\rho^{S}}^{+\infty} e^{-\frac{1}{2} v^{2}} d v\right)\right),
\end{aligned}
$$

where the RHS is $H\left(\hat{a}_{\infty}^{e}\right)$. Since $H(x)$ is a strictly increasing function of $x, H^{-1}(R)$ exists, implying that $\hat{a}_{\infty}^{e}=H^{-1}(R)$. In the setting where $a^{e}$ cannot exceed $U$, the optimal number of surgical procedures is given by $\hat{a}^{e}=\min \left\{H^{-1}(R), U\right\}$ by the concavity of $\Pi\left(a^{e}\right)$.

e) Next, consider the dependence of the optimal number of procedures $\hat{a}^{e}(\phi)$ on a parameter $\phi=R, c, \tau, \pi^{N}, \pi^{S}, \mu^{S}, \mu^{N}$, and $\bar{\sigma}^{S}$. If $\hat{a}^{e}(\phi) \geq U$, then the derivative of $\Pi$ with respect to $\phi$ is 0 , trivially satisfying all the given relations. Hence, we only consider the cases when $\hat{a}^{e}(\phi)<U$. Since

$$
\frac{\partial \Pi}{\partial a^{e}}\left(\hat{a}^{e}(\phi), \phi\right)=0
$$

we have

$$
\frac{\partial^{2} \Pi}{\partial\left(a^{e}\right)^{2}} \frac{\partial \hat{a}^{e}}{\partial \phi}+\frac{\partial^{2} \Pi}{\partial a^{e} \partial \phi}=0
$$

and

$$
\frac{\partial \hat{a}^{e}}{\partial \phi}=-\frac{\frac{\partial^{2} \Pi}{\partial a^{e} \partial \phi}}{\frac{\partial^{2} \Pi}{\partial\left(a^{e}\right)^{2}}},
$$


14

Bavafa, Leys, Örmeci, and Savin: Managing Portfolio of Elective Surgical Procedures

so that the sign of $\frac{\partial \hat{a}^{e}}{\partial \phi}$ coincides with the sign of $\frac{\partial^{2} \Pi}{\partial a^{e} \partial \phi}$. Using (A71), we have $\frac{\partial^{2} \Pi}{\partial a^{e} \partial R}>0$ and $\frac{\partial^{2} \Pi}{\partial a^{e} \partial \pi^{N}}, \frac{\partial^{2} \Pi}{\partial a^{e} \partial \pi^{S}}<0$. Further, since the sign $\frac{\partial^{2} \Pi}{\partial a^{e} \partial c}$ and $\frac{\partial^{2} \Pi}{\partial a^{e} \partial \tau}$ is opposite to the sign of $\frac{\partial^{2} \Pi}{\partial\left(a^{e}\right)^{2}}$, we have $\frac{\partial^{2} \Pi}{\partial a^{e} \partial c}>0, \frac{\partial^{2} \Pi}{\partial a^{e} \partial \tau}>0$, so that $\frac{\partial \hat{a}^{e}}{\partial c}>0$ and $\frac{\partial \hat{a}^{e}}{\partial \tau}>0$. Now, consider, for any $\boldsymbol{\Xi}_{t}$,

$$
\begin{aligned}
& \frac{\partial}{\partial \mu^{N}}\left(\mu^{N} \int_{\alpha\left(a^{e}, \boldsymbol{\Xi}_{t}\right)}^{+\infty} e^{-\frac{1}{2} v^{2}} d v+\sqrt{Q} \frac{A^{(1)}\left(a^{e}, \boldsymbol{\Xi}_{t}\right)}{\sqrt{A^{(2)}\left(a^{e}, \mathbf{\Xi}_{t}\right)}} e^{-\frac{1}{2}\left(\alpha\left(a^{e}, \boldsymbol{\Xi}_{t}\right)\right)^{2}}\right)=\int_{\alpha\left(a^{e}, \boldsymbol{\Xi}_{t}\right)}^{+\infty} e^{-\frac{1}{2} v^{2}} d v+ \\
& \frac{\mu^{N} a^{e}}{\sum^{N}\left(a^{e}, \mathbf{\Xi}_{t}\right)} e^{-\frac{1}{2}\left(\alpha\left(a^{e}, \boldsymbol{\Xi}_{t}\right)\right)^{2}}+\frac{\sqrt{Q} A^{(1)}\left(a^{e}, \boldsymbol{\Xi}_{t}\right)\left(c-M^{N}\left(a^{e}, \boldsymbol{\Xi}_{t}\right)\right) a^{e}}{\sqrt{A^{(2)}\left(a^{e}, \boldsymbol{\Xi}_{t}\right)}\left(\Sigma^{N}\left(a^{e}, \boldsymbol{\Xi}_{t}\right)\right)^{2}} e^{-\frac{1}{2}\left(\frac{c-M^{N}\left(a^{e}, \boldsymbol{\Xi}_{t}\right)}{\Sigma^{N}\left(a^{e}, \boldsymbol{\Xi}_{t}\right)}\right)^{2}} .
\end{aligned}
$$

Note that $\sigma^{N}\left(a^{e}, \boldsymbol{\Xi}_{t}\right)=\sqrt{A^{(2)}(\cdot)}$, and $M^{N}\left(a^{e}, \boldsymbol{\Xi}_{t}\right)=a^{e} \mu^{N}+\sum_{T=0}^{L_{\max }} \xi_{t-T} p_{T}^{L}$. To show that the last two terms are positive even for the largest possible value of urgent procedures, $h$, we consider the following expression:

$$
\frac{a^{e}}{\Sigma^{N}\left(a^{e}, \boldsymbol{\Xi}_{t}\right)} e^{-\frac{1}{2}\left(\frac{c-M^{N}\left(a^{e}, \mathbf{\Xi}_{t}\right)}{\Sigma^{N}\left(a^{e}, \mathbf{\Xi}_{t}\right)}\right)^{2}}\left(\mu^{N}+\frac{A^{(1)}\left(a^{e}, \boldsymbol{\Xi}_{t}\right)\left(c-\left(a^{e}+h\right) \mu^{N}\right)}{A^{(2)}\left(a^{e}, \boldsymbol{\Xi}_{t}\right)}\right),
$$

which is positive if and only if

$$
\mu^{N}\left(A^{(2)}\left(a^{e}, \boldsymbol{\Xi}_{t}\right)-A^{(1)}\left(a^{e}, \boldsymbol{\Xi}_{t}\right) a^{e}\right)+A^{(1)}\left(a^{e}, \boldsymbol{\Xi}_{t}\right)\left(c-h \mu^{N}\right)>0,
$$

which is always true under the assumption $c>h \mu^{N}$, so that $\frac{\partial^{2} \Pi}{\partial a^{e} \partial \mu^{N}}<0$ and $\frac{\partial \hat{a}^{e}}{\partial \mu^{N}}<0$.

For $\frac{\partial \hat{a}^{e}}{\partial \mu^{S}}$, we consider, for any $\xi \in[l, h]$,

$$
\begin{aligned}
& \frac{\partial}{\partial \mu^{S}}\left(e^{-\frac{1}{2}\left(\frac{\tau}{\left(a^{e}+\xi\right) \bar{\sigma}^{S}}-\rho^{S}\right)^{2}}+\rho^{S} \int_{\frac{\tau}{\left(a^{e}+\xi\right) \bar{\sigma}^{S}}-\rho^{S}}^{+\infty} e^{-\frac{1}{2} v^{2}} d v\right) \\
= & \left(\frac{\tau}{\left(a^{e}+\xi\right) \bar{\sigma}^{S}}-\rho^{S}\right) e^{-\frac{1}{2}\left(\frac{\tau}{\left(a^{e}+\xi\right) \bar{\sigma}^{S}}-\rho^{S}\right)^{2}} \frac{1}{\bar{\sigma}^{S}}+\frac{1}{\bar{\sigma}^{S}} \int_{\frac{\tau}{\left(a^{e}+\xi\right) \bar{\sigma}^{S}}-\rho^{S}}^{+\infty} e^{-\frac{1}{2} v^{2}} d v+\frac{1}{\bar{\sigma}^{S}} \rho^{S} e^{-\frac{1}{2}\left(\frac{\tau}{\left(a^{e}+\xi\right) \bar{\sigma}^{S}}-\rho^{S}\right)^{2}} \\
= & \left(\frac{\tau}{\left(a^{e}+\xi\right) \bar{\sigma}^{S}}\right) e^{-\frac{1}{2}\left(\frac{\tau}{\left(a^{e}+\xi\right) \bar{\sigma}^{S}}-\rho^{S}\right)^{2}} \frac{1}{\bar{\sigma}^{S}}+\frac{1}{\bar{\sigma}^{S}} \int_{\frac{\tau}{\left(a^{e}+\xi\right) \bar{\sigma}^{S}}-\rho^{S}}^{+\infty} e^{-\frac{1}{2} v^{2}} d v>0 .
\end{aligned}
$$

Next,

$$
\begin{aligned}
& \frac{\partial}{\partial \bar{\sigma}^{S}}\left(\bar{\sigma}^{S}\left(e^{-\frac{1}{2}\left(\frac{\tau}{\left(a^{e}+\xi\right) \bar{\sigma}^{S}}-\rho^{S}\right)^{2}}+\rho^{S} \int_{\frac{\tau}{\left(a^{e}+\xi\right) \bar{\sigma}^{S}}-\rho^{S}}^{+\infty} e^{-\frac{1}{2} v^{2}} d v\right)\right) \\
= & \left(e^{-\frac{1}{2}\left(\frac{\tau}{\left(a^{e}+\xi\right) \bar{\sigma}^{S}}-\rho^{S}\right)^{2}}+\rho^{S} \int_{\frac{\tau}{\left(a^{e}+\xi\right) \bar{\sigma}^{S}}-\rho^{S}}^{+\infty} e^{-\frac{1}{2} v^{2}} d v\right) \\
+ & \bar{\sigma}^{S} \frac{\partial}{\partial \bar{\sigma}^{S}}\left(e^{-\frac{1}{2}\left(\frac{\tau}{\left(a^{e}+\xi\right) \bar{\sigma}^{S}}-\rho^{S}\right)^{2}}+\rho^{S} \int_{\frac{\tau}{\left(a^{e}+\xi\right) \bar{\sigma}^{S}}-\rho^{S}}^{+\infty} e^{-\frac{1}{2} v^{2}} d v\right)
\end{aligned}
$$




$$
\begin{aligned}
& =\left(e^{-\frac{1}{2}\left(\frac{\tau}{\left(a^{e}+\xi\right) \bar{\sigma}^{S}}-\rho^{S}\right)^{2}}+\rho^{S} \int_{\frac{\tau}{\left(a^{e}+\xi\right) \bar{\sigma}^{S}}-\rho^{S}}^{+\infty} e^{-\frac{1}{2} v^{2}} d v\right)+\bar{\sigma}^{S} \\
& \times\left(\left(\frac{\tau}{\left(a^{e}+\xi\right) \bar{\sigma}^{S}}\right) e^{-\frac{1}{2}\left(\frac{\tau}{\left(a^{e}+\xi\right) \bar{\sigma}^{S}}-\rho^{S}\right)^{2}} \frac{1}{\bar{\sigma}^{S}}\left(\frac{\tau}{\left(a^{e}+\xi\right) \bar{\sigma}^{S}}-\rho^{S}\right)-\frac{\rho^{S}}{\bar{\sigma}^{S}} \int_{\frac{\tau}{\left(a^{e}+\xi\right) \bar{\sigma}^{S}}-\rho^{S}}^{+\infty} e^{-\frac{1}{2} v^{2}} d v\right) \\
& =e^{-\frac{1}{2}\left(\frac{\tau}{\left(a^{e}+\xi\right) \bar{\sigma}^{S}}-\rho^{S}\right)^{2}}\left(1+\left(\frac{\tau}{\left(a^{e}+\xi\right) \bar{\sigma}^{S}}-\rho^{S}\right)\left(\frac{\tau}{\left(a^{e}+\xi\right) \bar{\sigma}^{S}}\right)\right)>0
\end{aligned}
$$

for $a^{e}+h \leq \frac{\tau}{\mu^{S}}$, which is true under the assumption $R<H(\hat{h}-h)$.

f) Finally, consider cdf functions for the daily number of urgent procedures, $\Phi^{1}(\xi)$ and $\Phi^{2}(\xi)$ such that $\Phi^{1}(\xi) \leq \Phi^{2}(\xi), \forall \xi \in[l, h]$, and let $\hat{a}^{e, 1}$ and $\hat{a}^{e, 2}$ be the optimal daily numbers of elective procedures under $\Phi^{1}(\xi)$ and $\Phi^{2}(\xi)$, respectively. When $\hat{a}^{e, 1}$ or $\hat{a}^{e, 2}$ do not satisfy this constraint, the result is either trivial or it follows immediately from the result without the demand constraint. Accordingly, it is sufficient to show this result for the case without the demand constraint. Note that for any $\xi \in[l, h]$, both

$$
\frac{\partial g^{N}}{\partial a^{e}}=\mu^{N} \int_{\alpha\left(a^{e}, \boldsymbol{\Xi}_{t}\right)}^{+\infty} e^{-\frac{1}{2} v^{2}} d v+\sqrt{Q} \frac{A^{(1)}\left(a^{e}, \boldsymbol{\Xi}_{t}\right)}{\sqrt{A^{(2)}\left(a^{e}, \boldsymbol{\Xi}_{t}\right)}} e^{-\frac{1}{2} \alpha\left(a^{e}, \boldsymbol{\Xi}_{t}\right)^{2}}
$$

and

$$
\frac{\partial g^{S}}{\partial a^{e}}=e^{-\frac{1}{2}\left(\frac{\tau}{\left(a^{e}+\xi\right) \bar{\sigma}^{S}}-\rho^{S}\right)^{2}}+\rho^{S} \int_{\frac{\tau}{\left(a^{e}+\xi\right) \bar{\sigma}^{S}}-\rho^{S}}^{+\infty} e^{-\frac{1}{2} v^{2}} d v
$$

are increasing functions of $\xi$.

The first-order stochastic dominance of $\Phi^{1}(\xi)$ over $\Phi^{2}(\xi)$ implies that

$$
\begin{aligned}
& \frac{\pi^{N}}{\sqrt{2 \pi}} \int_{\boldsymbol{\Xi}^{1}} d \boldsymbol{\Xi}_{t}^{1} \Phi^{1}\left(\boldsymbol{\Xi}_{t}^{1}\right)\left(\mu^{N} \int_{\alpha\left(a^{e}, \boldsymbol{\Xi}_{t}^{1}\right)}^{+\infty} e^{-\frac{1}{2} v^{2}} d v+\sqrt{Q} \frac{A^{(1)}\left(a^{e}, \boldsymbol{\Xi}_{t}^{1}\right)}{\sqrt{A^{(2)}\left(a^{e}, \boldsymbol{\Xi}_{t}^{1}\right)}} e^{-\frac{1}{2} \alpha\left(a^{e}, \boldsymbol{\Xi}_{t}^{1}\right)^{2}}\right) \\
& +\frac{\pi^{S} \bar{\sigma}^{S}}{\sqrt{2 \pi}}\left(\int_{l}^{h} d \Phi^{1}\left(\xi^{1}\right)\left(e^{-\frac{1}{2}\left(\frac{\tau}{\left(a^{e}+\xi^{1}\right) \bar{\sigma}^{S}}-\rho^{S}\right)^{2}}+\rho^{S} \int_{\frac{\tau}{\left(a^{e}+\xi^{1}\right) \bar{\sigma}^{S}}-\rho^{S}}^{+\infty} e^{-\frac{1}{2} v^{2}} d v\right)\right) \\
& \geq \frac{\pi^{N}}{\sqrt{2 \pi}} \int_{\boldsymbol{\Xi}^{2}} d \boldsymbol{\Xi}_{t}^{2} \Phi^{2}\left(\boldsymbol{\Xi}_{t}^{2}\right)\left(\mu^{N} \int_{\alpha\left(a^{e}, \boldsymbol{\Xi}_{t}^{2}\right)}^{+\infty} e^{-\frac{1}{2} v^{2}} d v+\sqrt{Q} \frac{A^{(1)}\left(a^{e}, \boldsymbol{\Xi}_{t}^{2}\right)}{\sqrt{A^{(2)}\left(a^{e}, \boldsymbol{\Xi}_{t}^{2}\right)}} e^{-\frac{1}{2} \alpha\left(a^{e}, \boldsymbol{\Xi}_{t}^{2}\right)^{2}}\right) \\
& +\frac{\pi^{S} \bar{\sigma}^{S}}{\sqrt{2 \pi}}\left(\int_{l}^{h} d \Phi^{2}\left(\xi^{2}\right)\left(e^{-\frac{1}{2}\left(\frac{\tau}{\left(a^{e}+\xi^{2}\right) \bar{\sigma}^{S}}-\rho^{S}\right)^{2}}+\rho^{S} \int_{\frac{\tau}{\left(a^{e}+\xi^{2}\right) \bar{\sigma}^{S}}-\rho^{S}}^{+\infty} e^{-\frac{1}{2} v^{2}} d v\right)\right) .
\end{aligned}
$$

Then, since 


$$
\begin{aligned}
R & =\frac{\pi^{N}}{\sqrt{2 \pi}} \int_{\boldsymbol{\Xi}^{1}} d \boldsymbol{\Xi}_{t}^{1} \Phi^{1}\left(\boldsymbol{\Xi}_{t}^{1}\right)\left(\mu^{N} \int_{\alpha\left(\hat{a}^{e, 1}, \boldsymbol{\Xi}_{t}^{1}\right)}^{+\infty} e^{-\frac{1}{2} v^{2}} d v+\sqrt{Q} \frac{A^{(1)}\left(\hat{a}^{e, 1}, \boldsymbol{\Xi}_{t}^{1}\right)}{\sqrt{A^{(2)}\left(\hat{a}^{e, 1}, \boldsymbol{\Xi}_{t}^{1}\right)}} e^{-\frac{1}{2} \alpha\left(\hat{a}^{e, 1}, \boldsymbol{\Xi}_{t}^{1}\right)^{2}}\right) \\
& +\frac{\pi^{S} \bar{\sigma}^{S}}{\sqrt{2 \pi}}\left(\int_{l}^{h} d \Phi^{1}\left(\xi^{1}\right)\left(e^{-\frac{1}{2}\left(\frac{\tau}{\left(\hat{a}^{e, 1}+\xi^{1}\right) \bar{\sigma}^{S}}-\rho^{S}\right)^{2}}+\rho^{S} \int_{\frac{\tau}{\left(\hat{a}^{e, 1}+\xi^{1}\right) \bar{\sigma}^{S}}-\rho^{S}}^{+\infty} e^{-\frac{1}{2} v^{2}} d v\right)\right),
\end{aligned}
$$

we have

$$
\begin{array}{r}
\frac{\pi^{N}}{\sqrt{2 \pi}} \int_{\Xi^{2}} d \boldsymbol{\Xi}_{t}^{2} \Phi^{2}\left(\boldsymbol{\Xi}_{t}^{2}\right)\left(\mu^{N} \int_{\alpha\left(\hat{a}^{e, 1}, \boldsymbol{\Xi}_{t}^{2}\right)}^{+\infty} e^{-\frac{1}{2} v^{2}} d v+\sqrt{Q} \frac{A^{(1)}\left(\hat{a}^{e, 1}, \boldsymbol{\Xi}_{t}^{2}\right)}{\sqrt{A^{(2)}\left(\hat{a}^{e, 1}, \boldsymbol{\Xi}_{t}^{2}\right)}} e^{-\frac{1}{2} \alpha\left(\hat{a}^{e, 1}, \boldsymbol{\Xi}_{t}^{2}\right)^{2}}\right) \\
+\frac{\pi^{S} \bar{\sigma}^{S}}{\sqrt{2 \pi}}\left(\int_{l}^{h} d \Phi^{2}\left(\xi^{2}\right)\left(e^{-\frac{1}{2}\left(\frac{\tau}{\left(\hat{a}^{e, 1}+\xi^{2}\right) \bar{\sigma}^{S}}-\rho^{S}\right)^{2}}+\rho^{S} \int_{\frac{\tau}{\left(\hat{a}^{e, 1}+\xi^{2}\right) \bar{\sigma}^{S}}-\rho^{S}}^{+\infty} e^{-\frac{1}{2} v^{2}} d v\right)\right) \leq R,
\end{array}
$$

so that $\hat{a}^{e, 1} \leq \hat{a}^{e, 2}$.

\section{Proof of Proposition 3}

a) We start by observing that, as (A49) and (A71) imply, the optimal daily profit value is given by

$$
\begin{aligned}
& \Pi\left(\hat{a}^{e}\right)=R \hat{a}^{e}+R \int_{l}^{h} \xi f^{u}(\xi) d \xi \\
& -\frac{\pi^{S} \bar{\sigma}^{S}}{\sqrt{2 \pi}}\left(\int_{l}^{h} d \xi f^{u}(\xi)\left(\hat{a}^{e}+\xi\right)\left(e^{-\frac{1}{2}\left(\frac{\tau}{\left(\hat{a}^{e}+\xi\right) \bar{\sigma}^{S}}-\rho^{S}\right)^{2}}-\left(\frac{\tau}{\left(\hat{a}^{e}+\xi\right) \bar{\sigma}^{S}}-\rho^{S}\right) \int_{\frac{\tau}{\left(\hat{a}^{e}+\xi\right) \bar{\sigma}^{S}}-\rho^{S}}^{+\infty} e^{-\frac{1}{2} v^{2}} d v\right)\right) \\
& -\frac{\pi^{N}}{\sqrt{2 \pi}} \int_{\boldsymbol{\Xi}^{u}} d \boldsymbol{\Xi}_{t} F^{u}\left(\boldsymbol{\Xi}_{t}\right) \Sigma^{N}\left(a^{e}, \boldsymbol{\Xi}_{t}\right)\left(e^{-\frac{1}{2}\left(\frac{c-M^{N}\left(a^{e}, \boldsymbol{\Xi}_{t}\right)}{\Sigma^{N}\left(a^{e}, \boldsymbol{\Xi}_{t}\right)}\right)^{2}}-\left(\frac{c-M^{N}\left(a^{e}, \boldsymbol{\Xi}_{t}\right)}{\Sigma^{N}\left(a^{e}, \boldsymbol{\Xi}_{t}\right)}\right) \int_{\frac{c-M^{N}\left(a^{e}, \boldsymbol{\Xi}_{t}\right)}{\Sigma^{N}\left(a^{e}, \boldsymbol{\Xi}_{t}\right)}}^{+\infty} e^{-\frac{1}{2} v^{2}} d v\right) \\
& =\hat{a}^{e} \frac{\partial \Pi}{\partial \hat{a}^{e}}+\frac{\pi^{S} \tau}{\sqrt{2 \pi}} \int_{l}^{h} d \xi f^{u}(\xi) \int_{\frac{\tau}{\left(\hat{a}^{e}+\xi\right) \bar{\sigma}^{S}}-\rho^{S}}^{+\infty} e^{-\frac{1}{2} v^{2}} d v+\frac{\pi^{N} c}{\sqrt{2 \pi}} \int_{\Xi^{u}} d \boldsymbol{\Xi}_{t} F^{u}\left(\boldsymbol{\Xi}_{t}\right) \int_{\frac{c-M^{N}\left(a^{e}, \boldsymbol{\Xi}_{t}\right)}{\Sigma^{N}\left(a^{e}, \mathbf{\Xi}_{t}\right)}}^{+\infty} e^{-\frac{1}{2} v^{2}} d v \\
& +\int_{l}^{h} d \xi f^{u}(\xi) \xi\left(R-\frac{\pi^{S} \bar{\sigma}^{S}}{\sqrt{2 \pi}}\left(e^{-\frac{1}{2}\left(\frac{\tau}{\left(\hat{a}^{e}+\xi\right) \bar{\sigma}^{S}}-\rho^{S}\right)^{2}}+\rho^{S} \int_{\frac{\tau}{\left(\hat{a}^{e}+\xi\right) \bar{\sigma}^{S}}-\rho^{S}}^{+\infty} e^{-\frac{1}{2} v^{2}} d v\right)\right) \\
& -\frac{\pi^{N}}{\sqrt{2 \pi}} \int_{\boldsymbol{\Xi}^{u}} d \boldsymbol{\Xi}_{t} F^{u}\left(\boldsymbol{\Xi}_{t}\right)\left(\frac{\sqrt{Q}}{\sqrt{A^{(2)}\left(\hat{a}^{e}, \boldsymbol{\Xi}_{t}\right)}}\left(A^{(2)}\left(\hat{a}^{e}, \boldsymbol{\Xi}_{t}\right)-A^{(1)}\left(\hat{a}^{e}, \boldsymbol{\Xi}_{t}\right)\right) e^{-\frac{1}{2}\left(\alpha\left(\hat{a}^{e}, \boldsymbol{\Xi}_{t}\right)\right)^{2}}\right. \\
& \left.-\sum_{T=0}^{L_{\max }} \xi_{t-T} p_{T}^{L} \int_{\alpha\left(\hat{a}^{e}, \Xi_{t}\right)}^{+\infty} e^{-\frac{1}{2} v^{2}} d v\right)
\end{aligned}
$$




$$
\begin{aligned}
= & \frac{\pi^{S} \tau}{\sqrt{2 \pi}} \int_{l}^{h} d \xi f^{u}(\xi) \int_{\frac{\tau}{\left(\hat{a}^{e}+\xi\right) \bar{\sigma}^{S}}-\rho^{S}}^{+\infty} e^{-\frac{1}{2} v^{2}} d v+\frac{\pi^{N} c}{\sqrt{2 \pi}} \int_{\Xi^{u}} d \boldsymbol{\Xi}_{t} F^{u}\left(\boldsymbol{\Xi}_{t}\right) \int_{\frac{c-M^{N}\left(a^{e}, \boldsymbol{\Xi}_{t}\right)}{\Sigma^{N}\left(a^{e}, \mathbf{\Xi}_{t}\right)}}^{+\infty} e^{-\frac{1}{2} v^{2}} d v \\
& +\int_{l}^{h} d \xi f^{u}(\xi) \xi\left(R-\frac{\pi^{S} \bar{\sigma}^{S}}{\sqrt{2 \pi}}\left(e^{-\frac{1}{2}\left(\frac{\tau}{\left(\hat{a}^{e}+\xi\right) \bar{\sigma}^{S}}-\rho^{S}\right)^{2}}+\rho^{S} \int_{\frac{\tau}{\left(\hat{a}^{e}+\xi\right) \bar{\sigma}^{S}}-\rho^{S}}^{+\infty} e^{-\frac{1}{2} v^{2}} d v\right)\right) \\
& -\frac{\pi^{N}}{\sqrt{2 \pi}} \int_{\Xi^{u}} d \Xi_{t} F^{u}\left(\boldsymbol{\Xi}_{t}\right)\left(\frac{\sqrt{Q}}{\sqrt{A^{(2)}\left(\hat{a}^{e}, \mathbf{\Xi}_{t}\right)}}\left(A^{(2)}\left(\hat{a}^{e}, \boldsymbol{\Xi}_{t}\right)-A^{(1)}\left(\hat{a}^{e}, \boldsymbol{\Xi}_{t}\right)\right) e^{-\frac{1}{2}\left(\alpha\left(\hat{a}^{e}, \boldsymbol{\Xi}_{t}\right)\right)^{2}}\right. \\
& \left.-\sum_{T=0}^{L_{\max }} \xi_{t-T} p_{T}^{L} \int_{\alpha\left(\hat{a}^{e}, \Xi_{t}\right)}^{+\infty} e^{-\frac{1}{2} v^{2}} d v\right) .
\end{aligned}
$$

Since $\frac{\partial \Pi}{\partial \hat{a}^{e}}=0$ for $\hat{a}^{e}<U$, we have the following from (A71):

$$
\begin{aligned}
R & =\frac{\pi^{N}}{\sqrt{2 \pi}} \int_{\Xi^{u}} d \boldsymbol{\Xi}_{t} F^{u}\left(\boldsymbol{\Xi}_{t}\right)\left(\mu^{N} \int_{\alpha\left(\hat{a}^{e}, \Xi_{t}\right)}^{+\infty} e^{-\frac{1}{2} v^{2}} d v+\sqrt{Q} \frac{A^{(1)}\left(\hat{a}^{e}, \boldsymbol{\Xi}_{t}\right)}{\sqrt{A^{(2)}\left(\hat{a}^{e}, \mathbf{\Xi}_{t}\right)}} e^{-\frac{1}{2} \alpha\left(\hat{a}^{e}, \boldsymbol{\Xi}_{t}\right)^{2}}\right) \\
& +\frac{\pi^{S} \bar{\sigma}^{S}}{\sqrt{2 \pi}} \int_{l}^{h} d \xi f^{u}(\xi)\left(e^{-\frac{1}{2}\left(\frac{\tau}{\left(\hat{a}^{e}+\xi\right) \bar{\sigma}^{S}}-\rho^{S}\right)^{2}}+\rho^{S} \int_{\frac{\tau}{\left(\hat{a}^{e}+\xi\right) \bar{\sigma}^{S}}-\rho^{S}}^{+\infty} e^{-\frac{1}{2} v^{2}} d v\right) .
\end{aligned}
$$

In a similar way, we get for $\hat{a}_{S}^{e}<U$,

$$
R=\frac{\pi^{S} \bar{\sigma}^{S}}{\sqrt{2 \pi}} \int_{l}^{h} d \xi f^{u}(\xi)\left(e^{-\frac{1}{2}\left(\frac{\tau}{\left(\hat{a}_{S}^{e}+\xi\right) \bar{\sigma}^{S}}-\rho^{S}\right)^{2}}+\rho^{S} \int_{\frac{\tau}{\left(\hat{a}_{S}^{e}+\xi\right) \bar{\sigma}^{S}}-\rho^{S}}^{+\infty} e^{-\frac{1}{2} v^{2}} d v\right) .
$$

By concavity of $\Pi$ and $\Pi_{S}$, equations (A92) and (A93) imply that $\hat{a}^{e} \leq \hat{a}_{S}^{e}$. We also have $\hat{a}^{e} \leq U$ and $\hat{a}_{S}^{e} \leq U$. Then:

$$
\Pi\left(\hat{a}_{S}^{e}\right) \leq \Pi\left(\hat{a}^{e}\right) \leq \Pi_{S}\left(\hat{a}^{e}\right) \leq \Pi_{S}\left(\hat{a}_{S}^{e}\right)
$$

where the first inequality follows from the optimality of $\hat{a}^{e}$, the second - from the fact that $\Pi\left(a^{e}\right) \leq$ $\Pi_{S}\left(a^{e}\right)$ for any $a^{e}$, the third one - from the optimality of $\hat{a}_{S}^{e}$ for $\Pi_{S}\left(a^{e}\right)$. Note that the gap between $\Pi\left(\hat{a}_{S}^{e}\right)$ and $\Pi_{S}\left(\hat{a}_{S}^{e}\right)$ in the case with the demand constraint will always be smaller than that in the case without the demand constraint. Hence, we focus on the latter case, and ignore the demand constraint in the rest of the proof.

Let

$$
\begin{aligned}
\Pi_{S}\left(a^{e}\right)= & R a^{e}+R \int_{l}^{h} \xi f^{u}(\xi) d \xi \\
& -\frac{\pi^{S} \bar{\sigma}^{S}}{\sqrt{2 \pi}}\left(\int_{l}^{h} d \xi f^{u}(\xi)\left(a^{e}+\xi\right)\left(e^{-\frac{1}{2}\left(\frac{\tau}{\left(a^{e}+\xi\right) \bar{\sigma}^{S}}-\rho^{S}\right.}\right)^{2}\right.
\end{aligned}
$$




$$
\left.\left.-\left(\frac{\tau}{\left(a^{e}+\xi\right) \bar{\sigma}^{S}}-\rho^{S}\right) \int_{\frac{\tau}{\left(a^{e}+\xi\right) \bar{\sigma}^{S}}-\rho^{S}}^{+\infty} e^{-\frac{1}{2} v^{2}} d v\right)\right)
$$

be the profit value in the absence of the penalty cost associated with the recovery bed capacity. In addition, we observe that:

$$
\begin{aligned}
& \Pi_{S}\left(\hat{a}_{S}^{e}\right)=R \hat{a}_{S}^{e}+R \int_{l}^{h} \xi f^{u}(\xi) d \xi \\
& -\frac{\pi^{S} \bar{\sigma}^{S}}{\sqrt{2 \pi}}\left(\int _ { l } ^ { h } d \xi f ^ { u } ( \xi ) ( \hat { a } _ { S } ^ { e } + \xi ) \left(e^{-\frac{1}{2}\left(\frac{\tau}{\left(\hat{a}_{S}^{e}+\xi\right) \bar{\sigma}^{S}}-\rho^{S}\right)^{2}}\right.\right. \\
& \left.\left.-\left(\frac{\tau}{\left(\hat{a}_{S}^{e}+\xi\right) \bar{\sigma}^{S}}-\rho^{S}\right) \int_{\frac{\tau}{\left(\hat{a}_{S}^{e}+\xi\right) \bar{\sigma}^{S}}-\rho^{S}}^{+\infty} e^{-\frac{1}{2} v^{2}} d v\right)\right) \\
& =\hat{a}_{S}^{e} \frac{\partial \prod_{S}}{\partial \hat{a}_{S}^{e}}+\frac{\pi^{S} \tau}{\sqrt{2 \pi}} \int_{l}^{h} d \xi f^{u}(\xi) \int_{\frac{\tau}{\left(\hat{a}^{e}+\xi\right) \bar{\sigma}^{S}}-\rho^{S}}^{+\infty} e^{-\frac{1}{2} v^{2}} d v+ \\
& +\int_{l}^{h} d \xi f^{u}(\xi) \xi\left(R-\frac{\pi^{S} \bar{\sigma}^{S}}{\sqrt{2 \pi}}\left(e^{-\frac{1}{2}\left(\frac{\tau}{\left(\hat{a}^{e}+\xi\right) \bar{\sigma}^{S}}-\rho^{S}\right)^{2}}+\rho^{S} \int_{\frac{\tau}{\left(\hat{a}^{e}+\xi\right) \bar{\sigma}^{S}}-\rho^{S}}^{+\infty} e^{-\frac{1}{2} v^{2}} d v\right)\right) \\
& =\frac{\pi^{S} \tau}{\sqrt{2 \pi}} \int_{l}^{h} d \xi f^{u}(\xi) \int_{\frac{\tau}{\left(\hat{a}^{e}+\xi\right) \bar{\sigma}^{S}}-\rho^{S}}^{+\infty} e^{-\frac{1}{2} v^{2}} d v+ \\
& +\int_{l}^{h} d \xi f^{u}(\xi) \xi\left(R-\frac{\pi^{S} \bar{\sigma}^{S}}{\sqrt{2 \pi}}\left(e^{-\frac{1}{2}\left(\frac{\tau}{\left(\hat{a}^{e}+\xi\right) \bar{\sigma}^{S}}-\rho^{S}\right)^{2}}+\rho^{S} \int_{\frac{\tau}{\left(\hat{a}^{e}+\xi\right) \bar{\sigma}^{S}}-\rho^{S}}^{+\infty} e^{-\frac{1}{2} v^{2}} d v\right)\right),
\end{aligned}
$$

since $\frac{\partial \Pi_{S}}{\partial \hat{a}_{S}^{e}}=0$. In addition, second inequality of (56) implies that $\frac{\partial \Pi_{S}}{\partial a^{e}}\left(\frac{\tau}{\mu^{S}}-h\right)<0$, so that

$$
\hat{a}_{S}^{e}<\frac{\tau}{\mu^{S}}-h,
$$

by concavity of $\Pi_{S}$. Consider the performance gap associated with using $\hat{a}_{S}^{e}$ instead of $\hat{a}^{e}$ :

$$
\frac{\Pi\left(\hat{a}^{e}\right)-\Pi\left(\hat{a}_{S}^{e}\right)}{\Pi\left(\hat{a}^{e}\right)}=1-\frac{\Pi\left(\hat{a}_{S}^{e}\right)}{\Pi\left(\hat{a}^{e}\right)} \leq 1-\frac{\Pi\left(\hat{a}_{S}^{e}\right)}{\Pi_{S}\left(\hat{a}_{S}^{e}\right)}=\frac{\Pi_{S}\left(\hat{a}_{S}^{e}\right)-\Pi\left(\hat{a}_{S}^{e}\right)}{\Pi_{S}\left(\hat{a}_{S}^{e}\right)},
$$

where

$$
\begin{aligned}
\Pi_{S}\left(\hat{a}_{S}^{e}\right)-\Pi\left(\hat{a}_{S}^{e}\right)=\frac{\pi^{N}}{\sqrt{2 \pi}} \int_{\boldsymbol{\Xi}^{u}} d \boldsymbol{\Xi}_{t} F^{u}\left(\boldsymbol{\Xi}_{t}\right) \Sigma^{N}\left(a^{e}, \boldsymbol{\Xi}_{t}\right) \\
\left(e^{-\frac{1}{2}\left(\alpha\left(\hat{a}_{S}^{e}, \boldsymbol{\Xi}_{t}\right)\right)^{2}}-\alpha\left(\hat{a}_{S}^{e}, \boldsymbol{\Xi}_{t}\right) \int_{\alpha\left(\hat{a}_{S}^{e}, \boldsymbol{\Xi}_{t}\right)}^{+\infty} e^{-\frac{1}{2} v^{2}} d v\right)
\end{aligned}
$$


by (A91) and (A96), whereas $\Pi_{S}\left(\hat{a}_{S}^{e}\right)$ is given by (A96). Note that we will suppress the dependence of all functions on $\boldsymbol{\Xi}_{t}$.

Further, since

$$
\left(e^{-\frac{1}{2}(Z)^{2}}-Z \int_{Z}^{+\infty} e^{-\frac{1}{2} v^{2}} d v\right)
$$

is a decreasing function of $Z, \alpha\left(a^{e}\right)$ is a decreasing function of $a^{e}$, and $\hat{a}_{S}^{e}<\frac{\tau}{\mu^{S}}-h$, we have

$$
e^{-\frac{1}{2} \alpha\left(\hat{a}_{S}^{e}, \cdot\right)^{2}}-\alpha\left(\hat{a}_{S}^{e}, \cdot\right) \int_{\alpha\left(\hat{a}_{S}^{e}, \cdot\right)}^{+\infty} e^{-\frac{1}{2} v^{2}} d v<e^{-\frac{1}{2} \alpha\left(\frac{\tau}{\mu^{S}}-h, \cdot\right)^{2}}-\alpha\left(\frac{\tau}{\mu^{S}}-h, \cdot\right) \int_{\alpha\left(\frac{\tau}{\mu^{S}}-h, \cdot\right)}^{+\infty} e^{-\frac{1}{2} v^{2}} d v .
$$

Thus, for the right-hand side of (A98) we have:

$$
\begin{aligned}
& \frac{\pi^{N}}{\sqrt{2 \pi}} \int_{\Xi^{u}} d \boldsymbol{\Xi}_{t} F^{u}\left(\boldsymbol{\Xi}_{t}\right) \sqrt{Q A^{(2)}\left(\hat{a}_{S}^{e}, \cdot\right)}\left(e^{-\frac{1}{2}\left(\alpha\left(\hat{a}_{S}^{e}, \cdot\right)\right)^{2}}-\alpha\left(\hat{a}_{S}^{e}, \cdot\right) \int_{\alpha\left(\hat{a}_{S}^{e}, \cdot\right)}^{+\infty} e^{-\frac{1}{2} v^{2}} d v\right)
\end{aligned}
$$

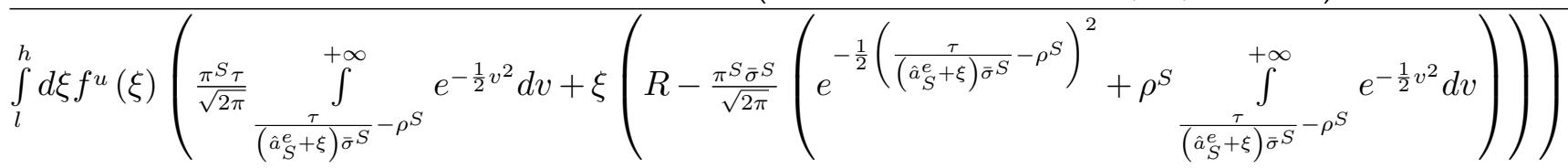

$$
\begin{aligned}
& \frac{\pi^{N}}{\sqrt{2 \pi}} \int_{\boldsymbol{\Xi}^{u}} d \boldsymbol{\Xi}_{t} F^{u}\left(\boldsymbol{\Xi}_{t}\right) \sqrt{Q A^{(2)}\left(\frac{\tau}{\mu^{S}}-h, \cdot\right)}\left(e^{-\frac{1}{2}\left(\alpha\left(\frac{\tau}{\mu^{S}}-h, \cdot\right)\right)^{2}}-\alpha\left(\frac{\tau}{\mu^{S}}-h, \cdot\right) \int_{\alpha\left(\frac{\tau}{\mu^{S}}-h, \cdot\right)}^{+\infty} e^{-\frac{1}{2} v^{2}} d v\right) \\
& \leq \frac{\int_{l}^{h} d \xi f^{u}(\xi)\left(\frac{\pi^{S} \tau}{\sqrt{2 \pi}} \int_{\frac{\tau}{\left(\hat{a}_{S}^{e}+\xi\right) \bar{\sigma}^{S}}-\rho^{S}}^{+\infty} e^{-\frac{1}{2} v^{2}} d v+\xi\left(R-\frac{\pi^{S} \bar{\sigma}^{S}}{\sqrt{2 \pi}}\left(e^{-\frac{1}{2}\left(\frac{\tau}{\left(\hat{a}_{S}^{e}+\xi\right) \bar{\sigma}^{S}}-\rho^{S}\right)^{2}}+\rho^{S} \int_{\frac{\tau}{\left(\hat{a}_{S}^{e}+\xi\right) \bar{\sigma}^{S}}-\rho^{S}}^{+\infty} e^{\left.-\frac{1}{2} v^{2} d v\right)}\right)\right)\right.}{,}
\end{aligned}
$$

since $A^{(2)}\left(a^{e}, \cdot\right)$ is also increasing in $a^{e}$.

Now noting that:

$$
\begin{aligned}
\alpha\left(a^{e}, \boldsymbol{\Xi}_{t}\right) & =\frac{c-M^{N}\left(a^{e}, \boldsymbol{\Xi}_{t}\right)}{\Sigma^{N}\left(a^{e}, \mathbf{\Xi}_{t}\right)}, \\
M^{N}\left(\mathbf{a}^{e}, \boldsymbol{\Xi}_{t}\right) & =a^{e} \mu^{N}+\sum_{T=0}^{L_{\max }^{i}} \xi_{t-T} p_{T}^{L}, \\
\left(\Sigma^{N}\left(\mathbf{a}^{e}, \boldsymbol{\Xi}_{t}\right)\right)^{2} & =\left(\sum_{k=1}^{K} q_{k}^{2}\right)\left(\sum_{T=0+1}^{L_{\max }}\left(a^{e}+\xi_{t-T}\right)^{2} p_{T}^{L}\left(1-p_{T}^{L}\right)\right)=Q A^{(2)}\left(a^{e}, \boldsymbol{\Xi}_{t}\right),
\end{aligned}
$$

the expression in the numerator of (A102) can be written as:

$$
\int_{\boldsymbol{\Xi}^{u}} d \boldsymbol{\Xi}_{t} F^{u}\left(\boldsymbol{\Xi}_{t}\right) \sqrt{Q A^{(2)}\left(\frac{\tau}{\mu^{S}}-h, \cdot\right)} \times
$$




$$
\begin{aligned}
& \left(e^{-\frac{1}{2}\left(\alpha\left(\frac{\tau}{\mu^{S}}-h, \cdot\right)\right)^{2}}-\left(\frac{c-\left(\frac{\tau}{\mu^{S}}-h\right) \mu^{N}-\sum_{T=0}^{L_{\max }} \xi_{t-T} p_{T}^{L}}{\sqrt{Q A^{(2)}\left(\frac{\tau}{\mu^{S}}-h, \cdot\right)}} \int_{\alpha\left(\frac{\tau}{\mu^{S}}-h, \cdot\right)}^{+\infty} e^{-\frac{1}{2} v^{2}} d v\right)\right. \\
& =\int_{\boldsymbol{\Xi}^{u}} d \boldsymbol{\Xi}_{t} F^{u}\left(\boldsymbol{\Xi}_{t}\right) \sqrt{Q A^{(2)}\left(\frac{\tau}{\mu^{S}}-h, \cdot\right)} e^{-\frac{1}{2}\left(\alpha\left(\frac{\tau}{\mu^{S}}-h, \cdot\right)\right)^{2}} \\
& -\int_{\boldsymbol{\Xi}^{u}} d \boldsymbol{\Xi}_{t} F^{u}\left(\boldsymbol{\Xi}_{t}\right)\left(\left(c-\left(\frac{\tau}{\mu^{S}}-h\right) \mu^{N}-\sum_{T=0}^{L_{\max }} \xi_{t-T} p_{T}^{L}\right) \int_{\alpha\left(\frac{\tau}{\mu^{S}}-h, \cdot\right)}^{+\infty} e^{-\frac{1}{2} v^{2}} d v\right) \\
& =\int_{\Xi^{u}} d \boldsymbol{\Xi}_{t} F^{u}\left(\boldsymbol{\Xi}_{t}\right) \sqrt{Q A^{(2)}\left(\frac{\tau}{\mu^{S}}-h, \cdot\right)} e^{-\frac{1}{2}\left(\alpha\left(\frac{\tau}{\mu^{S}}-h, \cdot\right)\right)^{2}} \\
& -\left(c-\left(\frac{\tau}{\mu^{S}}-h\right) \mu^{N}\right) \int_{\Xi^{u}} d \boldsymbol{\Xi}_{t} F^{u}\left(\boldsymbol{\Xi}_{t}\right) \int^{+\infty} e^{-\frac{1}{2} v^{2}} d v \\
& \alpha\left(\frac{\tau}{\mu^{S}}-h, \cdot\right) \\
& +\int_{\boldsymbol{\Xi}^{u}} d \boldsymbol{\Xi}_{t} F^{u}\left(\boldsymbol{\Xi}_{t}\right) \sum_{T=0}^{L_{\max }} p_{T}^{L} \xi_{t-T} \int_{(\tau)}^{+\infty} e^{-\frac{1}{2} v^{2}} d v \\
& \alpha\left(\frac{\tau}{\mu^{S}}-h, \cdot\right) \\
& =I_{2}^{N}-\left(c-\left(\frac{\tau}{\mu^{S}}-h\right) \mu^{N}\right) I_{0}^{N}+I_{1}^{N},
\end{aligned}
$$

where

$$
\begin{aligned}
& I_{0}^{N}=\int_{\boldsymbol{\Xi}^{u}} d \boldsymbol{\Xi}_{t} F^{u}\left(\boldsymbol{\Xi}_{t}\right) \int_{\alpha\left(\frac{\tau}{\mu^{S}}-h, \cdot\right)}^{+\infty} e^{-\frac{1}{2} v^{2}} d v, \\
& I_{1}^{N}=\int_{\Xi^{u}} d \boldsymbol{\Xi}_{t} F^{u}\left(\boldsymbol{\Xi}_{t}\right) \sum_{T=0}^{L_{\max }} p_{T}^{L} \xi_{t-T} \int_{\alpha\left(\frac{\tau}{\mu^{S}}-h, \cdot\right)}^{+\infty} e^{-\frac{1}{2} v^{2}} d v, \\
& I_{2}^{N}=\int_{\Xi^{u}} d \boldsymbol{\Xi}_{t} F^{u}\left(\boldsymbol{\Xi}_{t}\right) \sqrt{Q A^{(2)}\left(\frac{\tau}{\mu^{S}}-h, \cdot\right)} e^{-\frac{1}{2}\left(\alpha\left(\frac{\tau}{\mu^{S}}-h, \cdot\right)\right)^{2}},
\end{aligned}
$$

as defined by (49), (50) and (51) in the paper, respectively. Now we consider the denominator of (A102). Since

$$
\left(e^{-\frac{1}{2}(Z)^{2}}+\rho^{S} \int_{Z}^{+\infty} e^{-\frac{1}{2} v^{2}} d v\right)
$$

is a decreasing function of $Z$ for $Z>0, \frac{\tau}{\left(a_{S}^{e}+\xi\right) \bar{\sigma}^{S}}-\rho^{S}$ is a decreasing function of $a_{S}^{e}$, and $\frac{\tau}{\left(a_{S}^{e}+\xi\right) \bar{\sigma}^{S}}-$ $\rho^{S}>0$ by (A97), we have, for any $\xi \in[l, h]$, 


$$
\begin{gathered}
e^{-\frac{1}{2}\left(\frac{\tau}{\left(\hat{a}_{S}^{e}+\xi\right) \bar{\sigma} S}-\rho^{S}\right)^{2}}+\rho^{S} \int_{\frac{\tau}{\left(\hat{a}_{S}^{e}+\xi\right) \bar{\sigma}^{S}}-\rho^{S}}^{+\infty} e^{-\frac{1}{2} v^{2}} d v \\
\leq e^{-\frac{1}{2}\left(\frac{\tau}{\left(\frac{\tau}{\left.\mu^{S}-h+\xi\right) \bar{\sigma}^{S}}-\rho^{S}\right.}\right)^{2}}+\rho^{S} \int_{\frac{\tau}{\left(\frac{\tau}{\left.\mu^{S}-h+\xi\right)}\right)^{S}}-\rho^{S}}^{+\infty} e^{-\frac{1}{2} v^{2}} d v .
\end{gathered}
$$

Then, for the denominator of (A102) we have:

$$
\begin{aligned}
& \int_{l}^{h} d \xi f^{u}(\xi)\left(\frac{\pi^{S} \tau}{\sqrt{2 \pi}} \int_{\frac{\tau}{\left(\hat{a}_{S}^{e}+\xi\right) \bar{\sigma}^{S}}-\rho^{S}}^{+\infty} e^{-\frac{1}{2} v^{2}} d v\right. \\
& \left.+\xi\left(R-\frac{\pi^{S} \bar{\sigma}^{S}}{\sqrt{2 \pi}}\left(e^{-\frac{1}{2}\left(\frac{\tau}{\left(\hat{a}_{S}^{e}+\xi\right) \bar{\sigma}^{S}}-\rho^{S}\right)^{2}}+\rho^{S} \int_{\frac{\tau}{\left(\hat{a}_{S}^{e}+\xi\right) \bar{\sigma}^{S}}-\rho^{S}}^{+\infty} e^{-\frac{1}{2} v^{2}} d v\right)\right)\right) \\
& \geq \int_{l}^{h} d \xi f^{u}(\xi)\left(\frac{\pi^{S} \tau}{\sqrt{2 \pi}} \int_{\frac{\tau}{\left(\hat{a}_{S}^{e}+\xi\right) \bar{\sigma}^{S}}-\rho^{S}}^{+\infty} e^{-\frac{1}{2} v^{2}} d v\right. \\
& \left.+\xi\left(R-\frac{\pi^{S} \bar{\sigma}^{S}}{\sqrt{2 \pi}}\left(e^{-\frac{1}{2}\left(\frac{\tau}{\left(\frac{\tau}{\mu^{S}}-h+\xi\right) \bar{\sigma}^{S}}-\rho^{S}\right)^{2}}+\rho^{S} \int_{\frac{\tau}{\left(\frac{\tau}{\mu^{S}}-h+\xi\right) \bar{\sigma}^{S}}-\rho^{S}}^{+\infty} e^{-\frac{1}{2} v^{2}} d v\right)\right)\right) \\
& =\frac{\pi^{S} \tau}{\sqrt{2 \pi}} \int_{l}^{h} d \xi f^{u}(\xi) \int_{\frac{\tau}{\left(\hat{a}_{S}^{e}+\xi\right) \bar{\sigma}^{S}}-\rho^{S}}^{+\infty} e^{-\frac{1}{2} v^{2}} d v \\
& +\int_{l}^{h} d \xi f^{u}(\xi) \xi\left(R-\frac{\pi^{S} \bar{\sigma} S}{\sqrt{2 \pi}}\left(e^{-\frac{1}{2}\left(\frac{\tau}{\left(\frac{\tau}{\mu^{S}}-h+\xi\right) \bar{\sigma}^{S}}-\rho^{S}\right)^{2}}+\rho_{\frac{\tau}{\left(\frac{\tau}{\mu^{S}}-h+\xi\right) \bar{\sigma}^{S}}-\rho^{S}}^{+\infty} e^{-\frac{1}{2} v^{2}} d v\right)\right) \\
& =\frac{\tau}{\mu^{S}}\left(\int_{l}^{h} d \xi f^{u}(\xi) \frac{\pi^{S} \mu^{S}}{\sqrt{2 \pi}} \int_{\frac{\tau}{\left(\hat{a}_{S}^{e}+\xi\right) \bar{\sigma}^{S}}-\rho^{S}}^{+\infty} e^{-\frac{1}{2} v^{2}} d v\right)
\end{aligned}
$$




$$
+\int_{l}^{h} d \xi f^{u}(\xi) \xi\left(R-\frac{\pi^{S} \bar{\sigma}^{S}}{\sqrt{2 \pi}}\left(e^{-\frac{1}{2}\left(\frac{\tau}{\left(\frac{\tau}{\mu^{S}}-h+\xi\right) \bar{\sigma}^{S}}-\rho^{S}\right)^{2}}+\rho_{\frac{\tau}{\left(\frac{\tau}{\mu^{S}}-h+\xi\right) \bar{\sigma}^{S}}-\rho^{S}}^{+\infty} e^{-\frac{1}{2} v^{2}} d v\right)\right)
$$

Now, according to (A93), we have

$$
R-\frac{\pi^{S} \bar{\sigma}^{S}}{\sqrt{2 \pi}} \int_{l}^{h} d \xi f^{u}(\xi) e^{-\frac{1}{2}\left(\frac{\tau}{\left(\hat{a}_{S}^{e}+\xi\right) \bar{\sigma}^{S}}-\rho^{S}\right)^{2}}=\frac{\pi^{S} \bar{\sigma}^{S}}{\sqrt{2 \pi}} \rho^{S} \int_{l}^{h} d \xi f^{u}(\xi) \int_{\frac{\tau}{\left(\hat{a}_{S}^{e}+\xi\right) \bar{\sigma}^{S}}-\rho^{S}}^{+\infty} e^{-\frac{1}{2} v^{2}} d v
$$

Note that (A113) is equivalent to

$$
\int_{l}^{h} d \xi f^{u}(\xi)\left(R-\frac{\pi^{S} \bar{\sigma}^{S}}{\sqrt{2 \pi}} e^{-\frac{1}{2}\left(\frac{\tau}{\left(\hat{a}_{S}^{e}+\xi\right) \bar{\sigma}^{S}}-\rho^{S}\right)^{2}}\right)=\int_{l}^{h} d \xi f^{u}(\xi) \frac{\pi^{S} \mu^{S}}{\sqrt{2 \pi}} \int_{\frac{\tau}{\left(\hat{a}_{S}^{e}+\xi\right) \bar{\sigma}^{S}}-\rho^{S}}^{+\infty} e^{-\frac{1}{2} v^{2}} d v .
$$

Thus, the right-hand side of (A112) is equivalent to

$$
\begin{aligned}
& \frac{\tau}{\mu^{S}}\left(\int_{l}^{h} d \xi f^{u}(\xi)\left(R-\frac{\pi^{S} \bar{\sigma}^{S}}{\sqrt{2 \pi}} e^{\left.-\frac{1}{2}\left(\frac{\tau}{\left(\hat{a}_{S}^{e}+\xi\right) \bar{\sigma}^{S}}-\rho^{S}\right)^{2}\right)}\right)\right. \\
+ & \int_{l}^{h} d \xi f^{u}(\xi) \xi\left(R-\frac{\pi^{S} \bar{\sigma}^{S}}{\sqrt{2 \pi}}\left(e^{-\frac{1}{2}\left(\frac{\tau}{\left(\frac{\tau}{\mu^{S}}-h+\xi\right) \bar{\sigma}^{S}}-\rho^{S}\right)^{2}}+\rho_{\frac{\tau}{\left(\frac{\tau}{\mu^{S}}-h+\xi\right) \bar{\sigma}^{S}}-\rho^{S}}^{+\infty} e^{-\frac{1}{2} v^{2}} d v\right)\right)
\end{aligned}
$$

Note that since

$$
e^{-\frac{1}{2}\left(\frac{\tau}{\left(a_{S}^{e}+\xi\right) \bar{\sigma} S}-\rho^{S}\right)^{2}}
$$

is an increasing function of $a_{S}^{e}$ for $\frac{\tau}{\left(a_{S}^{e}+\xi\right) \bar{\sigma}^{S}}-\rho^{S}>0$, we have, since $\frac{\tau}{\left(\hat{a}_{S}^{e}+h\right) \bar{\sigma}^{S}}-\rho^{S}>0$ (by (A97)),

$$
\begin{aligned}
& \frac{\tau}{\mu^{S}}\left(\int_{l}^{h} d \xi f^{u}(\xi)\left(R-\frac{\pi^{S} \bar{\sigma}^{S}}{\sqrt{2 \pi}} e^{-\frac{1}{2}\left(\frac{\tau}{\left(\hat{a}_{S}^{e}+\xi\right) \bar{\sigma}^{S}}-\rho^{S}\right)^{2}}\right)\right)
\end{aligned}
$$

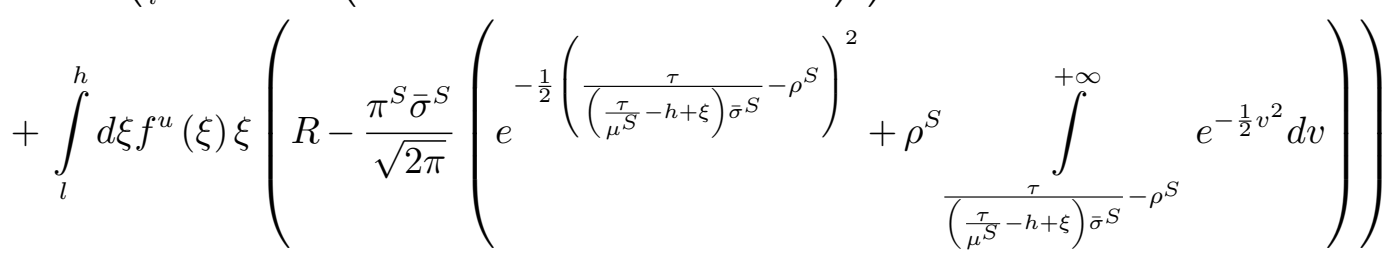

$$
\begin{aligned}
& \geq \frac{\tau}{\mu^{S}}\left(\int_{l}^{h} d \xi f^{u}(\xi)\left(R-\frac{\pi^{S} \bar{\sigma}^{S}}{\sqrt{2 \pi}} e^{-\frac{1}{2}\left(\frac{\tau}{\left(\frac{\tau}{\mu^{S}}-h+\xi\right) \bar{\sigma}^{S}}-\rho^{S}\right)^{2}}\right)\right)
\end{aligned}
$$




$$
+\int_{l}^{h} d \xi f^{u}(\xi) \xi\left(R-\frac{\pi^{S} \bar{\sigma}^{S}}{\sqrt{2 \pi}}\left(e^{-\frac{1}{2}\left(\frac{\tau}{\left(\frac{\tau}{\mu^{S}}-h+\xi\right) \bar{\sigma}^{S}}-\rho^{S}\right)^{2}}+\rho_{\frac{\tau}{\left(\frac{\tau}{\left.\mu^{S}-h+\xi\right) \bar{\sigma}^{S}}-\rho^{S}\right.}}^{+\infty} \int^{-\frac{1}{2} v^{2}} d v\right)\right)
$$

Then, the denominator of (A102) can be limited from below by

$$
\begin{aligned}
& \frac{\tau}{\mu^{S}}\left(\int_{l}^{h} d \xi f^{u}(\xi)\left(R-\frac{\pi^{S} \bar{\sigma}^{S}}{\sqrt{2 \pi}} e^{-\frac{1}{2}\left(\frac{\tau}{\left(\frac{\tau}{\left.\mu^{S}-h+\xi\right)} \bar{\sigma}^{S}\right.}-\rho^{S}\right)^{2}}\right)\right) \\
& +\int_{l}^{h} d \xi f^{u}(\xi) \xi\left(R-\frac{\pi^{S} \bar{\sigma}^{S}}{\sqrt{2 \pi}}\left(e^{-\frac{1}{2}\left(\frac{\tau}{\left(\frac{\tau}{\mu^{S}}-h+\xi\right) \bar{\sigma}^{S}}-\rho^{S}\right)^{2}}+\rho_{\frac{\tau}{\left(\frac{\tau}{\mu^{S}}-h+\xi\right) \bar{\sigma}^{S}}-\rho^{S}}^{+\infty} e^{-\frac{1}{2} v^{2}} d v\right)\right) \\
& =\frac{\tau}{\mu^{S}} R-\frac{\tau}{\mu^{S}}\left(\int_{l}^{h} d \xi f^{u}(\xi)\left(\frac{\pi^{S} \bar{\sigma}^{S}}{\sqrt{2 \pi}} e^{-\frac{1}{2}\left(\frac{\tau}{\left(\frac{\tau}{\mu^{S}}-h+\xi\right) \bar{\sigma}^{S}}-\rho^{S}\right)^{2}}\right)\right)
\end{aligned}
$$

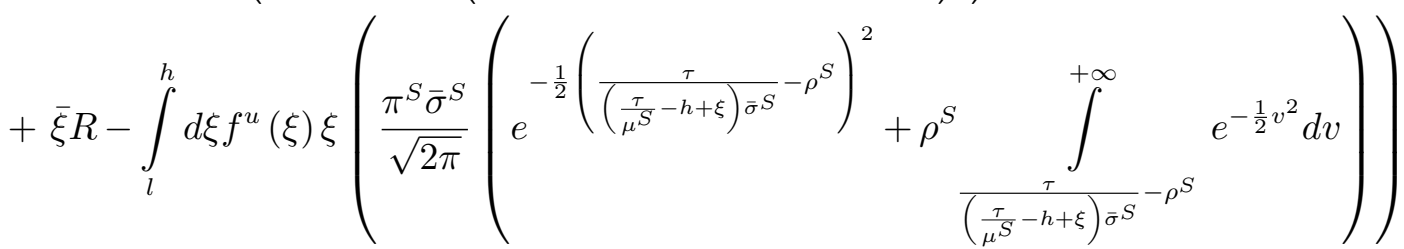

$$
\begin{aligned}
& =\left(\bar{\xi}+\frac{\tau}{\mu^{S}}\right) R-\frac{\tau}{\mu^{S}}\left(\frac{\pi^{S} \bar{\sigma}^{S}}{\sqrt{2 \pi}}\right) J_{1}^{S}-\left(\frac{\pi^{S} \bar{\sigma}^{S}}{\sqrt{2 \pi}}\right) J_{2}^{S}-\left(\frac{\pi^{S} \mu^{S}}{\sqrt{2 \pi}}\right) I_{2}^{S},
\end{aligned}
$$

where

$$
\begin{aligned}
J_{1}^{S} & =\int_{l}^{h} d \xi f^{u}(\xi) e^{-\frac{1}{2}\left(\frac{\tau}{\left(\frac{\tau}{\mu^{S}}-h+\xi\right) \bar{\sigma}^{S}}-\rho^{S}\right)^{2}}, \\
J_{2}^{S} & =\int_{l}^{h} d \xi f^{u}(\xi) \xi e^{-\frac{1}{2}\left(\frac{\tau}{\left(\frac{\tau}{\mu^{S}}-h+\xi\right) \bar{\sigma}^{S}}-\rho^{S}\right)^{2}}, \\
I_{2}^{S} & =\int_{l}^{h} d \xi f^{u}(\xi) \xi \int_{\tau}^{+\infty} e^{-\frac{1}{2} v^{2} d v} \\
& =\sqrt{\frac{\pi}{2}} \int_{l}^{h} d \xi f^{u}(\xi) \xi \operatorname{erfc}\left(\frac{1}{\sqrt{2}}\left(\frac{\tau+\rho^{S}}{\left(\frac{\tau}{\mu^{S}}-h+\xi\right) \bar{\sigma}^{S}}-\rho^{S}\right)\right) .
\end{aligned}
$$

Then, as it follows from (A102), the performance gap associated with setting the daily number of elective procedures to $\hat{a}_{S}^{e}$ satisfies 


$$
\begin{aligned}
& \frac{\Pi\left(\hat{a}^{e}\right)-\Pi\left(\hat{a}_{S}^{e}\right)}{\Pi\left(\hat{a}^{e}\right)} \leq \frac{\pi^{N}}{\sqrt{2 \pi}} \frac{I_{2}^{N}-\left(c-\left(\frac{\tau}{\mu^{S}}-h\right) \mu^{N}\right) I_{0}^{N}+I_{1}^{N}}{\left(\bar{\xi}+\frac{\tau}{\mu^{S}}\right) R-\frac{\tau}{\mu^{S}}\left(\frac{\pi^{S} \bar{\sigma}^{S}}{\sqrt{2 \pi}}\right) J_{1}^{S}-\left(\frac{\pi^{S} \bar{\sigma}^{S}}{\sqrt{2 \pi}}\right) J_{2}^{S}-\left(\frac{\pi^{S} \mu^{S}}{\sqrt{2 \pi}}\right) I_{2}^{S}} \\
= & \left(\frac{\pi^{N}}{\pi^{S} \bar{\sigma}^{S}}\right) \frac{I_{2}^{N}-\left(c-\left(\frac{\tau}{\mu^{S}}-h\right) \mu^{N}\right) I_{0}^{N}+I_{1}^{N}}{\sqrt{2 \pi}\left(\bar{\xi}+\frac{\tau}{\mu^{S}}\right)\left(\frac{R}{\pi^{S} \bar{\sigma}^{S}}\right)-\frac{\tau}{\mu^{S}} J_{1}^{S}-J_{2}^{S}-\rho^{S} I_{2}^{S}} .
\end{aligned}
$$

b) Consider two cdf functions $\Phi^{1}(\xi)$ and $\Phi^{1}(\xi)$, both strictly increasing on $\xi \in[l, h]$ and satisfying (58)-(60). Then, consider the numerator of the expression for $\epsilon^{S},(\mathrm{~A} 102)$,

$$
\begin{aligned}
\frac{\pi^{N}}{\sqrt{2 \pi}} \int_{\boldsymbol{\Xi}^{1}} d \boldsymbol{\Xi}_{t}^{1} \Phi^{1} & \left(\boldsymbol{\Xi}_{t}^{1}\right) \sqrt{Q A^{(2)}\left(\frac{\tau}{\mu^{S}}-h, \boldsymbol{\Xi}_{t}^{1}\right)} \\
& \times\left(e^{-\frac{1}{2}\left(\alpha\left(\frac{\tau}{\mu^{S}}-h, \boldsymbol{\Xi}_{t}^{1}\right)\right)^{2}}-\alpha\left(\frac{\tau}{\mu^{S}}-h, \boldsymbol{\Xi}_{t}^{1}\right) \int_{\alpha\left(\frac{\tau}{\mu^{S}}-h, \boldsymbol{\Xi}_{t}^{1}\right)}^{+\infty} e^{-\frac{1}{2} v^{2}} d v\right) .
\end{aligned}
$$

Note that both $A^{(2)}\left(\frac{\tau}{\mu^{S}}-h, \boldsymbol{\Xi}\right)$ and

$$
e^{-\frac{1}{2}\left(\alpha\left(\frac{\tau}{\mu^{S}}-h, \boldsymbol{\Xi}\right)\right)^{2}}-\alpha\left(\frac{\tau}{\mu^{S}}-h, \boldsymbol{\Xi}\right) \int_{\alpha\left(\frac{\tau}{\mu^{S}}-h, \boldsymbol{\Xi}\right)}^{+\infty} e^{-\frac{1}{2} v^{2}} d v
$$

are increasing functions of each component of $\boldsymbol{\Xi}=\left(\xi_{t-L_{\max }}, \ldots \xi_{t}\right)$. From the first-order stochastic dominance of $\Phi^{1}(\xi)$ over $\Phi^{2}(\xi)$, it follows that

$$
\begin{aligned}
& \frac{\pi^{N}}{\sqrt{2 \pi}} \int_{\boldsymbol{\Xi}^{1}} d \boldsymbol{\Xi}_{t}^{1} \Phi^{1}\left(\boldsymbol{\Xi}_{t}^{1}\right) \sqrt{Q A^{(2)}\left(\frac{\tau}{\mu^{S}}-h, \boldsymbol{\Xi}_{t}^{1}\right)}\left(e^{-\frac{1}{2}\left(\alpha\left(\frac{\tau}{\mu^{S}}-h, \boldsymbol{\Xi}_{t}^{1}\right)\right)^{2}}-\alpha\left(\frac{\tau}{\mu^{S}}-h, \boldsymbol{\Xi}_{t}^{1}\right) \int_{\alpha\left(\frac{\tau}{\mu^{S}}-h, \boldsymbol{\Xi}_{t}^{1}\right)}^{+\infty} e^{-\frac{1}{2} v^{2}} d v\right) \\
& \geq \frac{\pi^{N}}{\sqrt{2 \pi}} \int_{\Xi^{2}} d \boldsymbol{\Xi}_{t}^{2} \Phi^{2}\left(\boldsymbol{\Xi}_{t}^{2}\right) \sqrt{Q A^{(2)}\left(\frac{\tau}{\mu^{S}}-h, \boldsymbol{\Xi}_{t}^{2}\right)} \\
& \times\left(e^{-\frac{1}{2}\left(\alpha\left(\frac{\tau}{\mu^{S}}-h, \boldsymbol{\Xi}_{t}^{2}\right)\right)^{2}}-\alpha\left(\frac{\tau}{\mu^{S}}-h, \boldsymbol{\Xi}_{t}^{2} \int_{\alpha\left(\frac{\tau}{\mu^{S}}-h, \boldsymbol{\Xi}_{t}^{2}\right)}^{+\infty} e^{-\frac{1}{2} v^{2}} d v\right) .\right.
\end{aligned}
$$

Now, consider the denominator of the expression for $\epsilon^{S}$, (A122),

$$
\sqrt{2 \pi}\left(\bar{\xi}+\frac{\tau}{\mu^{S}}\right)\left(\frac{R}{\pi^{S} \bar{\sigma}^{S}}\right)-\frac{\tau}{\mu^{S}} J_{1}^{S}-J_{2}^{S}-\rho^{S} I_{2}^{S} .
$$


Note that each of the integrals in this expression, $J_{1}^{S}, J_{2}^{S}$, and $I_{2}^{S}$, is the expected value of a monotone function of $\xi$. Consider, for example, $I_{2}^{S}$,

$$
\sqrt{\frac{\pi}{2}} \int_{l}^{h} d \xi f^{u}(\xi) \xi \operatorname{erfc}\left(\frac{1}{\sqrt{2}}\left(\frac{\tau}{\left(\frac{\tau}{\mu^{S}}-h+\xi\right) \bar{\sigma}^{S}}-\rho^{S}\right)\right) .
$$

Given that $\xi \operatorname{erfc}\left(\frac{1}{\sqrt{2}}\left(\frac{\tau}{\left(\frac{\tau}{\mu^{S}}-h+\xi\right) \bar{\sigma}^{S}}-\rho^{S}\right)\right)$ is a monotone increasing function of $\xi$, the first-order stochastic dominance of $\Phi^{1}\left(\xi^{1}\right)$ over $\Phi^{2}\left(\xi^{2}\right)$ implies that

$$
\begin{aligned}
& \sqrt{\frac{\pi}{2}} \int_{l}^{h} d \Phi^{1}\left(\xi^{1}\right) \xi^{1} \operatorname{erfc}\left(\frac{1}{\sqrt{2}}\left(\frac{\tau}{\left(\frac{\tau}{\mu^{S}}-h+\xi^{1}\right) \bar{\sigma}^{S}}-\rho^{S}\right)\right) \\
\geq & \sqrt{\frac{\pi}{2}} \int_{l}^{h} d \Phi^{2}\left(\xi^{2}\right) \xi^{2} \operatorname{erfc}\left(\frac{1}{\sqrt{2}}\left(\frac{\tau}{\left(\frac{\tau}{\mu^{S}}-h+\xi^{2}\right) \bar{\sigma}^{S}}-\rho^{S}\right)\right) .
\end{aligned}
$$

Thus, the numerator of the expression for $\epsilon^{S}$ increases when evaluated using $\Phi^{1}\left(\xi^{1}\right)$ instead of $\Phi^{2}\left(\xi^{2}\right)$, while the denominator decreases. Hence, $\epsilon^{S}$ corresponding to $\Phi^{1}\left(\xi^{1}\right)$ is higher than that corresponding to $\Phi^{2}\left(\xi^{2}\right)$.

\section{Proof of Proposition 4}

a) Consider a portfolio of two procedure types $\left(a_{1}^{e}, a_{2}^{e}\right)$. Then, the expected surgery overtime on day $t$ can be computed as

$$
\begin{aligned}
& E\left[\left(D_{t}\left(a_{1}^{e}, a_{2}^{e}\right)-\tau\right)^{+}\right]=\frac{1}{\sqrt{2 \pi\left(a_{2}^{e}\right)^{2}\left(\bar{\sigma}_{2}^{S}\right)^{2}}} \int_{\tau}^{\infty}(x-\tau) e^{-\frac{\left(x-a_{1}^{e} \mu_{1}^{S}-a_{2}^{e} \mu_{2}^{S}\right)^{2}}{2\left(a_{2}^{e}\right)^{2}\left(\bar{\sigma}_{2}^{S}\right)^{2}}} d x \\
= & \frac{a_{2}^{e} \bar{\sigma}_{2}^{S}}{\sqrt{2 \pi}}\left(e^{-\frac{\left(\tau-a_{1}^{e} \mu_{1}^{S}-a_{2}^{e} \mu_{2}^{S}\right)^{2}}{2\left(a_{2}^{e}\right)^{2}\left(\bar{\sigma}_{2}^{S}\right)^{2}}}-\left(\frac{\tau-a_{1}^{e} \mu_{1}^{S}-a_{2}^{e} \mu_{2}^{S}}{a_{2}^{e} \bar{\sigma}_{2}^{S}}\right) \int_{\frac{\tau-a_{1}^{e} \mu_{1}^{S}-a_{2}^{e} \mu_{2}^{S}}{a_{2}^{e} \bar{\sigma}_{2}^{S}}}^{\infty} e^{-\frac{1}{2} v^{2}} d v\right) .
\end{aligned}
$$

Also, for the expected "overflow" in the number of occupied beds is

$$
E\left[\left(O_{t}\left(a_{1}^{e}, a_{2}^{e}\right)-c\right)^{+}\right]=\frac{a_{1}^{e} \bar{\sigma}_{1}^{N}}{\sqrt{2 \pi}}\left(e^{-\frac{\left(c-a_{1}^{e} \mu_{1}^{N}-a_{2}^{e} \mu_{2}^{N}\right)^{2}}{2\left(a_{1}^{e}\right)^{2}\left(\bar{\sigma}_{1}^{N}\right)^{2}}}-\left(\frac{c-a_{1}^{e} \mu_{1}^{N}-a_{2}^{e} \mu_{2}^{N}}{a_{1}^{e} \bar{\sigma}_{1}^{N}}\right) \int_{\frac{c-a_{1}^{e} \mu_{1}^{N}-a_{2}^{e} \mu_{2}^{N}}{a_{1}^{e} \bar{\sigma}_{1}^{N}}}^{\infty} e^{-\frac{1}{2} v^{2}} d v\right) .
$$


Thus, the daily expected hospital's profit is

$$
\begin{aligned}
\Pi\left(a_{1}^{e}, a_{2}^{e}\right) & =R_{1} a_{1}^{e}+R_{2} a_{2}^{e}-\frac{a_{2}^{e} \pi^{S} \bar{\sigma}_{2}^{S}}{\sqrt{2 \pi}}\left(e^{-\frac{\left(\tau-a_{1}^{e} \mu_{1}^{S}-a_{2}^{e} \mu_{2}^{S}\right)^{2}}{2\left(a_{2}^{e}\right)^{2}\left(\bar{\sigma}_{2}^{S}\right)^{2}}}-\left(\frac{\tau-a_{1}^{e} \mu_{1}^{S}-a_{2}^{e} \mu_{2}^{S}}{a_{2}^{e} \bar{\sigma}_{2}^{S}}\right) \int_{\frac{\tau-a_{1}^{e} \mu_{1}^{S}-a_{2}^{e} \mu_{2}^{S}}{a_{2}^{e} \bar{\sigma}_{2}^{S}}}^{\infty} e^{-\frac{1}{2} v^{2}} d v\right) \\
& -\frac{a_{1}^{e} \bar{\sigma}_{1}^{N} \pi^{N}}{\sqrt{2 \pi}}\left(e^{-\frac{\left(c-a_{1}^{e} \mu_{1}^{N}-a_{2}^{e} \mu_{2}^{N}\right)^{2}}{2\left(a_{1}^{e}\right)^{2}\left(\bar{\sigma}_{2}^{N}\right)^{2}}}-\left(\frac{c-a_{1}^{e} \mu_{1}^{N}-a_{2}^{e} \mu_{2}^{N}}{a_{1}^{e} \bar{\sigma}_{1}^{N}}\right) \int_{\frac{c-a_{1}^{e} \mu_{1}^{N}-a_{2}^{e} \mu_{2}^{N}}{a_{1}^{e} \bar{\sigma}_{1}^{N}}}^{\infty} e^{-\frac{1}{2} v^{2}} d v\right) \cdot(\mathrm{A} 131)
\end{aligned}
$$

Calculating the partial derivative of the objective function with respect to $a_{1}^{e}$ and $a_{2}^{e}$, we have

$$
\frac{\partial \Pi}{\partial a_{1}^{e}}=R_{1}-\frac{\pi^{N} \bar{\sigma}_{1}^{N}}{\sqrt{2 \pi}} e^{-\frac{1}{2}\left(\frac{c-a_{1}^{e} \mu_{1}^{N}-a_{2}^{e} \mu_{2}^{N}}{a_{1}^{e} \bar{\sigma}_{1}^{N}}\right)^{2}}-\frac{\pi^{N} \mu_{1}^{N}}{\sqrt{2 \pi}} \int_{\frac{c-a_{1}^{e} \mu_{1}^{N}-a_{2}^{e} \mu_{2}^{N}}{a_{1}^{e} \bar{\sigma}_{1}^{N}}}^{\infty} e^{-\frac{1}{2} v^{2}} d v-\frac{\pi^{S} \mu_{1}^{S}}{\sqrt{2 \pi}} \int_{\frac{\tau-a_{1}^{e} \mu_{1}^{S}-a_{2}^{e} \mu_{2}^{S}}{a_{2}^{e} \bar{\sigma}_{2}^{S}}}^{\infty} e^{-\frac{1}{2} v^{2}} d v,
$$

and

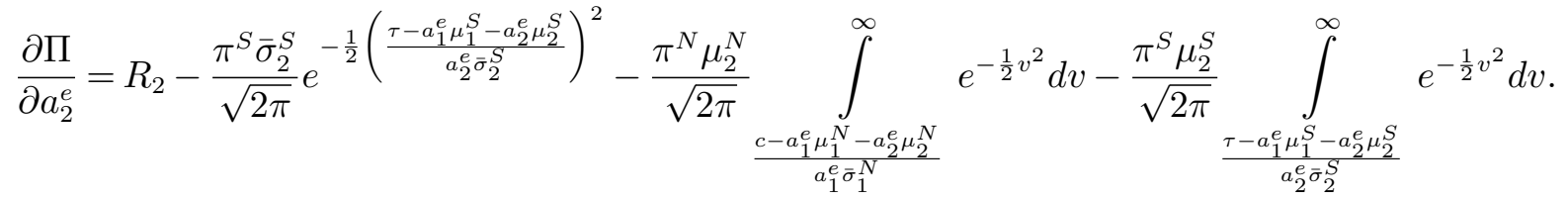

$\Pi\left(a_{1}^{e}, a_{2}^{e}\right)$ is concave if and only if

$$
\frac{\partial^{2} \Pi}{\partial\left(a_{1}^{e}\right)^{2}}<0
$$

and

$$
\left(\frac{\partial^{2} \Pi}{\partial a_{2}^{e} \partial a_{1}^{e}}\right)^{2} \leq\left(\frac{\partial^{2} \Pi}{\partial\left(a_{2}^{e}\right)^{2}}\right)\left(\frac{\partial^{2} \Pi}{\partial\left(a_{1}^{e}\right)^{2}}\right)
$$

Noting that:

$$
\begin{array}{r}
\frac{c-a_{1}^{e} \mu_{1}^{N}-a_{2}^{e} \mu_{2}^{N}}{a_{1}^{e} \bar{\sigma}_{1}^{N}}=\frac{c-a_{2}^{e} \mu_{2}^{N}}{a_{1}^{e} \bar{\sigma}_{1}^{N}}-\rho_{1}^{N}, \text { and } \\
\frac{\tau-a_{1}^{e} \mu_{1}^{S}-a_{2}^{e} \mu_{2}^{S}}{a_{2}^{e} \bar{\sigma}_{2}^{S}}=\frac{\tau-a_{1}^{e} \mu_{1}^{S}}{a_{2}^{e} \bar{\sigma}_{2}^{S}}-\rho_{2}^{S},
\end{array}
$$

we calculate the second order partial derivatives of the objective function with respect to $a_{1}^{e}$ and $a_{2}^{e}$ :

$\frac{\partial^{2} \Pi}{\partial\left(a_{1}^{e}\right)^{2}}=-\frac{\pi^{N} \bar{\sigma}_{1}^{N}}{\sqrt{2 \pi}}\left(\frac{c-a_{2}^{e} \mu_{2}^{N}}{a_{1}^{e} \bar{\sigma}_{1}^{N}}-\rho_{1}^{N}\right) \frac{c-a_{2}^{e} \mu_{2}^{N}}{\left(a_{1}^{e}\right)^{2} \bar{\sigma}_{1}^{N}} e^{-\frac{1}{2}\left(\frac{c-a_{2}^{e} \mu_{2}^{N}}{a_{1}^{e} \bar{\sigma}_{1}^{N}}-\rho_{1}^{N}\right)^{2}}-\frac{\pi^{N} \mu_{1}^{N}}{\sqrt{2 \pi}} \frac{c-a_{2}^{e} \mu_{2}^{N}}{\left(a_{1}^{e}\right)^{2} \bar{\sigma}_{1}^{N}} e^{-\frac{1}{2}\left(\frac{c-a_{2}^{e} \mu_{2}^{N}}{a_{1}^{e} \bar{\sigma}_{1}^{N}-\rho_{1}^{N}}\right)^{2}}$ 


$$
\begin{aligned}
& -\frac{\pi^{S} \mu_{1}^{S}}{\sqrt{2 \pi}} \frac{\mu_{1}^{S}}{a_{2}^{e} \bar{\sigma}_{2}^{S}} e^{-\frac{1}{2}\left(\frac{\tau-a_{1}^{e} \mu_{1}^{S}}{a_{2}^{e} \bar{\sigma}_{2}^{S}}-\rho_{2}^{S}\right)^{2}} \\
= & -\frac{\pi^{N} \bar{\sigma}_{1}^{N}}{\sqrt{2 \pi}}\left(\frac{c-a_{2}^{e} \mu_{2}^{N}}{\bar{\sigma}_{1}^{N}}\right)^{2} \frac{1}{\left(a_{1}^{e}\right)^{3}} e^{-\frac{1}{2}\left(\frac{c-a_{2}^{e} \mu_{2}^{N}}{a_{1}^{e} \bar{\sigma}_{1}^{N}}-\rho_{1}^{N}\right)^{2}}-\frac{\pi^{S} \mu_{1}^{S}}{\sqrt{2 \pi}} \frac{\mu_{1}^{S}}{a_{2}^{e} \bar{\sigma}_{2}^{S}} e^{-\frac{1}{2}\left(\frac{\tau-a_{1}^{e} \mu_{1}^{S}}{a_{2}^{e} \bar{\sigma}_{2}^{S}}-\rho_{2}^{S}\right)^{2}},
\end{aligned}
$$

which establishes (A134). Now we consider the other second order partial derivatives:

$$
\frac{\partial^{2} \Pi}{\partial\left(a_{2}^{e}\right)^{2}}=\frac{\pi^{S} \bar{\sigma}_{2}^{S}}{\sqrt{2 \pi}}\left(\frac{1}{\left(a_{2}^{e}\right)^{3}}\right)\left(\frac{\tau-a_{1}^{e} \mu_{1}^{S}}{\bar{\sigma}_{2}^{S}}\right)^{2} e^{-\frac{1}{2}\left(\frac{\tau-a_{1}^{e} \mu_{1}^{S}}{a_{2}^{e} \bar{\sigma}_{2}^{S}}-\rho_{2}^{S}\right)^{2}}-\frac{\pi^{N} \mu_{2}^{N}}{\sqrt{2 \pi}}\left(\frac{\mu_{2}^{N}}{a_{1}^{e} \bar{\sigma}_{1}^{N}}\right) e^{-\frac{1}{2}\left(\frac{c-a_{2}^{e} \mu_{2}^{N}}{a_{1}^{e} \bar{\sigma}_{1}^{N}}-\rho_{1}^{N}\right)^{2}},
$$

and

$$
\frac{\partial^{2} \Pi}{\partial a_{2}^{e} \partial a_{1}^{e}}=-\frac{\pi^{S} \bar{\sigma}_{2}^{S}}{\sqrt{2 \pi}}\left(\frac{\tau-a_{1}^{e} \mu_{1}^{S}}{a_{2}^{e} \bar{\sigma}_{2}^{S}}\right)\left(\frac{\mu_{1}^{S}}{a_{2}^{e} \bar{\sigma}_{2}^{S}}\right) e^{-\frac{1}{2}\left(\frac{\tau-a_{1}^{e} \mu_{1}^{S}}{a_{2}^{e} \bar{\sigma}_{2}^{S}}-\rho_{2}^{S}\right)^{2}}-\frac{\pi^{N} \mu_{2}^{N}}{\sqrt{2 \pi}}\left(\frac{c-a_{2}^{e} \mu_{2}^{N}}{\left(a_{1}^{e}\right)^{2} \bar{\sigma}_{1}^{N}}\right) e^{-\frac{1}{2}\left(\frac{c-a_{2}^{e} \mu_{2}^{N}}{a_{1}^{e} \bar{\sigma}_{1}^{N}}-\rho_{1}^{N}\right)^{2}} .
$$

Below we show that, in addition to (A134), we also have (A135). Since the penalty cost component of the profit function is the sum of two separate penalty cost components, it is sufficient to establish that each of those components is concave. In order to show that, it is sufficient to show that the statement of (A135) applies separately to each component. For the component associated with the recovery bed capacity, we get, using (A138)-(A140),

$$
\begin{aligned}
& \left(\frac{\pi^{N} \mu_{2}^{N}}{\sqrt{2 \pi}}\left(\frac{c-a_{2}^{e} \mu_{2}^{N}}{\left(a_{1}^{e}\right)^{2} \bar{\sigma}_{1}^{N}}\right) e^{-\frac{1}{2}\left(\frac{c-a_{2}^{e} \mu_{2}^{N}}{a_{1}^{e} \bar{\sigma}_{1}^{N}}-\rho_{1}^{N}\right)^{2}}\right)^{2} \\
& -\frac{\pi^{N} \bar{\sigma}_{1}^{N}}{\sqrt{2 \pi}}\left(\frac{c-a_{2}^{e} \mu_{2}^{N}}{\bar{\sigma}_{1}^{N}}\right)^{2} \frac{1}{\left(a_{1}^{e}\right)^{3}} e^{-\frac{1}{2}\left(\frac{c-a_{2}^{e} \mu_{2}^{N}}{a_{1}^{e} \bar{\sigma}_{1}^{N}}-\rho_{1}^{N}\right)^{2}} \times \frac{\pi^{N} \mu_{2}^{N}}{\sqrt{2 \pi}}\left(\frac{\mu_{2}^{N}}{a_{1}^{e} \bar{\sigma}_{1}^{N}}\right) e^{-\frac{1}{2}\left(\frac{c-a_{2}^{e} \mu_{2}^{N}}{a_{1}^{e} \bar{\sigma}_{1}^{N}}-\rho_{1}^{N}\right)^{2}},
\end{aligned}
$$

which is equal to 0 after some straightforward manipulation. Similarly, we have the following for the components associated with the OR capacity:

$$
\begin{aligned}
& \left(\frac{\pi^{S} \bar{\sigma}_{2}^{S}}{\sqrt{2 \pi}}\left(\frac{\tau-a_{1}^{e} \mu_{1}^{S}}{a_{2}^{e} \bar{\sigma}_{2}^{S}}\right)\left(\frac{\mu_{1}^{S}}{a_{2}^{e} \bar{\sigma}_{2}^{S}}\right) e^{-\frac{1}{2}\left(\frac{\tau-a_{1}^{e} \mu_{1}^{S}}{a_{2}^{e} \bar{\sigma}_{2}^{S}}-\rho_{2}^{S}\right)^{2}}\right)^{2} \\
& -\frac{\pi^{S} \bar{\sigma}_{2}^{S}}{\sqrt{2 \pi}} \frac{1}{\left(a_{2}^{e}\right)^{3}}\left(\frac{\tau-a_{1}^{e} \mu_{1}^{S}}{\bar{\sigma}_{2}^{S}}\right)^{2} e^{-\frac{1}{2}\left(\frac{\tau-a_{1}^{e} \mu_{1}^{S}}{a_{2}^{e} \bar{\sigma}_{2}^{S}}-\rho_{2}^{S}\right)^{2}} \times \frac{\pi^{S} \mu_{1}^{S}}{\sqrt{2 \pi}} \frac{\mu_{1}^{S}}{a_{2}^{e} \bar{\sigma}_{2}^{S}} e^{-\frac{1}{2}\left(\frac{\tau-a_{1}^{e} \mu_{1}^{S}}{a_{2}^{e} \bar{\sigma}_{2}^{S}}-\rho_{2}^{S}\right)^{2}},
\end{aligned}
$$

which is equal to 0 after some straightforward manipulation. Hence, $\Pi\left(a_{1}^{e}, a_{2}^{e}\right)$ is concave.

b) We first consider the case when there is no upper bound on the number of elective procedures. The optimal portfolio $\left(\hat{a}_{1}^{e}, \hat{a}_{2}^{e}\right)$ with $\hat{a}_{1}^{e}, \hat{a}_{2}^{e}>0$ must satisfy:

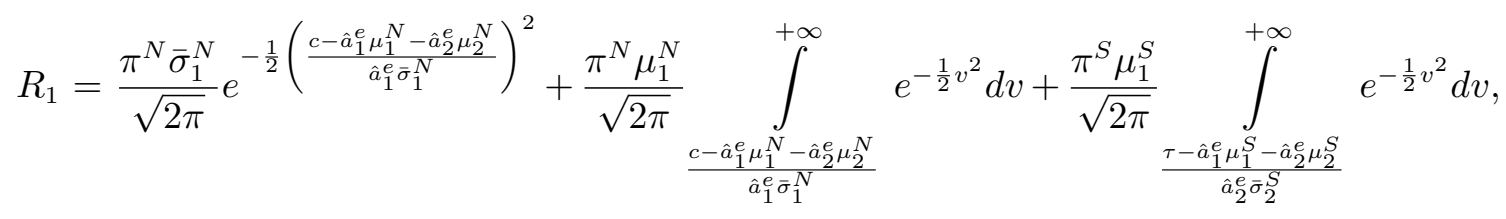




$$
R_{2}=\frac{\pi^{S} \bar{\sigma}_{2}^{S}}{\sqrt{2 \pi}} e^{-\frac{1}{2}\left(\frac{\tau-\hat{a}_{1}^{e} \mu_{1}^{S}-\hat{a}_{2}^{e} \mu_{2}^{S}}{\hat{a}_{2}^{e} \bar{\sigma}_{2}^{S}}\right)^{2}}+\frac{\pi^{N} \mu_{2}^{N}}{\sqrt{2 \pi}} \int_{\frac{c-\hat{a}_{1}^{e} \mu_{1}^{N}-\hat{a}_{2}^{e} \mu_{2}^{N}}{\hat{a}_{1}^{e} \bar{\sigma}_{1}^{N}}}^{+\infty} e^{-\frac{1}{2} v^{2}} d v+\frac{\pi^{S} \mu_{2}^{S}}{\sqrt{2 \pi}} \int_{\frac{\tau-\hat{a}_{1}^{e} \mu_{1}^{S}-\hat{a}_{2}^{e} \mu_{2}^{S}}{\hat{a}_{2}^{e} \bar{\sigma}_{2}^{S}}}^{+\infty} e^{-\frac{1}{2} v^{2}} d v .
$$

Defining

$$
\begin{aligned}
X_{a} & =\frac{c-\hat{a}_{1}^{e} \mu_{1}^{N}-\hat{a}_{2}^{e} \mu_{2}^{N}}{\hat{a}_{1}^{e} \bar{\sigma}_{1}^{N}}, \\
Y_{a} & =\frac{\tau-\hat{a}_{1}^{e} \mu_{1}^{S}-\hat{a}_{2}^{e} \mu_{2}^{S}}{\hat{a}_{2}^{e} \bar{\sigma}_{2}^{S}},
\end{aligned}
$$

we observe that (A143) and (A144) become

$$
R_{1}=\frac{\pi^{N} \bar{\sigma}_{1}^{N}}{\sqrt{2 \pi}} e^{-\frac{1}{2}\left(X_{a}\right)^{2}}+\frac{\pi^{N} \mu_{1}^{N}}{\sqrt{2 \pi}} \int_{X_{a}}^{+\infty} e^{-\frac{1}{2} v^{2}} d v+\frac{\pi^{S} \mu_{1}^{S}}{\sqrt{2 \pi}} \int_{Y_{a}}^{+\infty} e^{-\frac{1}{2} v^{2}} d v
$$

and

$$
R_{2}=\frac{\pi^{S} \bar{\sigma}_{2}^{S}}{\sqrt{2 \pi}} e^{-\frac{1}{2}\left(Y_{a}\right)^{2}}+\frac{\pi^{N} \mu_{2}^{N}}{\sqrt{2 \pi}} \int_{X_{a}}^{+\infty} e^{-\frac{1}{2} v^{2}} d v+\frac{\pi^{S} \mu_{2}^{S}}{\sqrt{2 \pi}} \int_{Y_{a}}^{+\infty} e^{-\frac{1}{2} v^{2}} d v
$$

Note that since the right-hand sides of (A147) and (A148) are decreasing functions of $X_{a}$ and $Y_{a}$, it is impossible to have positive values for both $X_{a}$ and $Y_{a}$ unless

$$
R_{1}<\frac{\pi^{N} \bar{\sigma}_{1}^{N}}{\sqrt{2 \pi}}+\frac{\pi^{N} \mu_{1}^{N}}{2}+\frac{\pi^{S} \mu_{1}^{S}}{2}
$$

and

$$
R_{2}<\frac{\pi^{S} \bar{\sigma}_{2}^{S}}{\sqrt{2 \pi}}+\frac{\pi^{N} \mu_{2}^{N}}{2}+\frac{\pi^{S} \mu_{2}^{S}}{2}
$$

Using (62) and (63), we can express (A149) and (A150) as

$$
1<\frac{\pi^{N}}{\sqrt{2 \pi} r^{N} \rho_{1}^{N}}+\frac{\pi^{N}}{2 r^{N}}+\frac{\pi^{S}}{2 r^{S}}
$$

and

$$
1<\frac{\pi^{S}}{\sqrt{2 \pi} r^{S} \rho_{2}^{S}}+\frac{\pi^{N}}{2 r^{N}}+\frac{\pi^{S}}{2 r^{S}}
$$

or as

$$
2<\frac{\pi^{N}}{r^{N}}+\frac{\pi^{S}}{r^{S}}+\sqrt{\frac{2}{\pi}} \min \left(\frac{\pi^{S}}{r^{S} \rho_{2}^{S}}, \frac{\pi^{N}}{r^{N} \rho_{1}^{N}}\right) .
$$

Using (62) and (63) in (A147) and (A148), we have

$$
\frac{\pi^{N} \bar{\sigma}_{1}^{N}}{\sqrt{2 \pi} R_{1}} e^{-\frac{1}{2}\left(X_{a}\right)^{2}}=\frac{\pi^{S} \bar{\sigma}_{2}^{S}}{\sqrt{2 \pi} R_{2}} e^{-\frac{1}{2}\left(Y_{a}\right)^{2}} .
$$


Let us define $Z$ such that

$$
\frac{1}{\sqrt{2 \pi}} e^{-\frac{1}{2} Z}=\frac{\pi^{N} \bar{\sigma}_{1}^{N}}{\sqrt{2 \pi} R_{1}} e^{-\frac{1}{2}\left(X_{a}\right)^{2}}=\frac{\pi^{S} \bar{\sigma}_{2}^{S}}{\sqrt{2 \pi} R_{2}} e^{-\frac{1}{2}\left(Y_{a}\right)^{2}}
$$

so that

$$
\begin{aligned}
& X_{a}=\sqrt{Z+2 \ln \left(\frac{\pi^{N}}{\rho_{1}^{N} r_{N}}\right)}, \\
& Y_{a}=\sqrt{Z+2 \ln \left(\frac{\pi^{S}}{\rho_{2}^{S} r_{S}}\right)} .
\end{aligned}
$$

Then, using (A148), we get

$$
\begin{aligned}
& \frac{1}{\sqrt{2 \pi}} e^{-\frac{1}{2} Z}=1-\frac{\pi^{N}}{\sqrt{2 \pi} r^{N}} \quad \int^{+\infty} e^{-\frac{1}{2} v^{2}} d v-\frac{\pi^{S}}{\sqrt{2 \pi} r^{S}} \int^{+\infty} e^{-\frac{1}{2} v^{2}} d v \\
& \sqrt{Z+2 \ln \left(\frac{\pi^{N}}{\rho_{1}^{N} r_{N}}\right)} \quad \sqrt{Z+2 \ln \left(\frac{\pi^{S}}{\rho_{2}^{S} r_{S}}\right)}
\end{aligned}
$$

or

$$
\frac{1}{\sqrt{2 \pi}} e^{-\frac{1}{2} Z}=1-\frac{\pi^{N}}{2 r^{N}} \operatorname{erfc}\left(\sqrt{\frac{1}{2} Z+\ln \left(\frac{\pi^{N}}{\rho_{1}^{N} r_{N}}\right)}\right)-\frac{\pi^{S}}{2 r^{S}} \operatorname{erfc}\left(\sqrt{\frac{1}{2} Z+\ln \left(\frac{\pi^{S}}{\rho_{2}^{S} r_{S}}\right)}\right) .
$$

Given that the left-hand side of (A159) is a decreasing function of $Z$ and the right-hand side an increasing one, the solution to (A159) is well-defined if, for $Z=2 \ln \left(\max \left(\frac{\rho_{1}^{N} r_{N}}{\pi^{N}}, \frac{\rho_{2}^{S} r_{S}}{\pi^{S}}\right)\right)$, we have the left-hand side value greater than the right-hand side value. Note that $X_{a}$ and $Y_{a}$ have real-valued solutions for $Z \geq 2 \ln \left(\frac{\rho_{1}^{N} r_{N}}{\pi^{N}}\right)$ and $Z \geq 2 \ln \left(\frac{\rho_{2}^{S} r_{S}}{\pi^{S}}\right)$ due to equations (A156) and (A157), respectively. Let, for example, $\frac{\rho_{1}^{N} r_{N}}{\pi^{N}}<\frac{\rho_{2}^{S} r_{S}}{\pi^{S}}$. Then, this condition can be expressed as

$$
\frac{1}{\sqrt{2 \pi}} \frac{\pi^{S}}{\rho_{2}^{S} r_{S}}>1-\frac{\pi^{N}}{2 r^{N}} \operatorname{erfc}\left(\sqrt{\ln \left(\frac{\pi^{N} \rho_{2}^{S} r_{S}}{\pi^{S} \rho_{1}^{N} r_{N}}\right)}\right)-\frac{\pi^{S}}{2 r^{S}}
$$

or

$$
\sqrt{\frac{2}{\pi}} \frac{\pi^{S}}{\rho_{2}^{S} r_{S}}+\frac{\pi^{N}}{r^{N}} \operatorname{erfc}\left(\sqrt{\ln \left(\frac{\pi^{N} \rho_{2}^{S} r_{S}}{\pi^{S} \rho_{1}^{N} r_{N}}\right)}\right)+\frac{\pi^{S}}{r^{S}}>2
$$

Note that the left-hand side of (A161) is smaller than the right-hand side of (A153). Thus, (A161) implies (A153). In the same fashion, for $\frac{\rho_{1}^{N} r_{N}}{\pi^{N}} \geq \frac{\rho_{2}^{S} r_{S}}{\pi^{S}}$, (A161) must be replaced by

$$
\sqrt{\frac{2}{\pi}} \frac{\pi^{N}}{\rho_{1}^{N} r_{N}}+\frac{\pi^{S}}{r^{S}} \operatorname{erfc}\left(\sqrt{\ln \left(\frac{\pi^{S} \rho_{1}^{N} r_{N}}{\pi^{N} \rho_{2}^{S} r_{S}}\right)}\right)+\frac{\pi^{N}}{r^{N}}>2
$$

which implies (A153).

Note that (A145) and (A146) are equivalent to

$$
\hat{a}_{1}^{e}=\frac{c\left(\mu_{2}^{S}+Y_{a} \bar{\sigma}_{2}^{S}\right)-\mu_{2}^{N} \tau}{\left(\mu_{1}^{N}+X_{a} \bar{\sigma}_{1}^{N}\right)\left(\mu_{2}^{S}+Y_{a} \bar{\sigma}_{2}^{S}\right)-\mu_{1}^{S} \mu_{2}^{N}},
$$




$$
\hat{a}_{2}^{e}=\frac{\tau\left(\mu_{1}^{N}+X_{a} \bar{\sigma}_{1}^{N}\right)-\mu_{1}^{S} c}{\left(\mu_{1}^{N}+X_{a} \bar{\sigma}_{1}^{N}\right)\left(\mu_{2}^{S}+Y_{a} \bar{\sigma}_{2}^{S}\right)-\mu_{1}^{S} \mu_{2}^{N}},
$$

or, using (A156)-(A157),

$$
\begin{gathered}
\hat{a}_{1}^{e}=\frac{c\left(\mu_{2}^{S}+\sqrt{Z+2 \ln \left(\frac{\pi^{S}}{\rho_{2}^{S} r_{S}}\right)} \bar{\sigma}_{2}^{S}\right)-\mu_{2}^{N} \tau}{\left(\mu_{1}^{N}+\sqrt{Z+2 \ln \left(\frac{\pi^{N}}{\rho_{1}^{N} r_{N}}\right)} \bar{\sigma}_{1}^{N}\right)\left(\mu_{2}^{S}+\sqrt{Z+2 \ln \left(\frac{\pi^{S}}{\rho_{2}^{S} r_{S}}\right)} \bar{\sigma}_{2}^{S}\right)-\mu_{1}^{S} \mu_{2}^{N}}, \\
\tau\left(\mu_{1}^{N}+\sqrt{\left.Z+2 \ln \left(\frac{\pi^{N}}{\rho_{1}^{N} r_{N}}\right) \bar{\sigma}_{1}^{N}\right)-\mu_{1}^{S} c}\right. \\
\hat{a}_{2}^{e}=\frac{\left.\mu_{1}^{N}+\sqrt{Z+2 \ln \left(\frac{\pi^{N}}{\rho_{1}^{N} r_{N}}\right)} \bar{\sigma}_{1}^{N}\right)\left(\mu_{2}^{S}+\sqrt{Z+2 \ln \left(\frac{\pi^{S}}{\rho_{2}^{S} r_{S}}\right)} \bar{\sigma}_{2}^{S}\right)-\mu_{1}^{S} \mu_{2}^{N}}{(\sqrt{Z}} .
\end{gathered}
$$

Dividing both the numerator and the denominator of the right-hand side of (A165) and (A166) by $\mu_{2}^{S} \mu_{1}^{N}$, and noting that

$$
\frac{\mu_{2}^{N}}{\mu_{2}^{S}}=\frac{R_{2} \mu_{2}^{N}}{R_{2} \mu_{2}^{S}}=\frac{r^{S}}{r^{N}}
$$

and

$$
\frac{\mu_{1}^{S}}{\mu_{1}^{N}}=\frac{R_{1} \mu_{1}^{S}}{R_{1} \mu_{1}^{N}}=\frac{r^{N}}{r^{S}}
$$

we get

$$
\begin{gathered}
\hat{a}_{1}^{e}=\frac{1}{\mu_{1}^{N}} \frac{c\left(1+\sqrt{Z+2 \ln \left(\frac{\pi^{S}}{\rho_{2}^{S} r_{S}}\right)} \frac{1}{\rho_{2}^{S}}\right)-\frac{r^{S}}{r^{N}} \tau}{\left(1+\sqrt{\left.Z+2 \ln \left(\frac{\pi^{N}}{\rho_{1}^{N} r_{N}}\right) \frac{1}{\rho_{1}^{N}}\right)\left(1+\sqrt{Z+2 \ln \left(\frac{\pi^{S}}{\rho_{2}^{S} r_{S}}\right)} \frac{1}{\rho_{2}^{S}}\right)-1}\right.}, \\
\hat{a}_{2}^{e}=\frac{1}{\mu_{2}^{S}} \frac{\tau\left(1+\sqrt{\left.Z+2 \ln \left(\frac{\pi^{N}}{\rho_{1}^{N} r_{N}}\right) \frac{1}{\rho_{1}^{N}}\right)-\frac{r^{N}}{r^{S}} c}\right.}{\left(1+\sqrt{Z+2 \ln \left(\frac{\pi^{N}}{\rho_{1}^{N} r_{N}}\right)} \frac{1}{\rho_{1}^{N}}\right)\left(1+\sqrt{Z+2 \ln \left(\frac{\pi^{S}}{\rho_{2}^{S} r_{S}}\right)} \frac{1}{\rho_{2}^{S}}\right)-1} .
\end{gathered}
$$

Then,

$$
\frac{\tau}{c}<\frac{r^{N}}{r^{S}}\left(1+\sqrt{Z+2 \ln \left(\frac{\pi^{S}}{\rho_{2}^{S} r_{S}}\right)} \frac{1}{\rho_{2}^{S}}\right)=m^{+},
$$

and

$$
\frac{\tau}{c}>\frac{r^{N}}{r^{S}} \frac{1}{\left(1+\sqrt{Z+2 \ln \left(\frac{\pi^{N}}{\rho_{1}^{N} r_{N}}\right)} \frac{1}{\rho_{1}^{N}}\right)}=m^{-}
$$

ensure that both numerators in (A165) and (A166) are positive, so that $\hat{a}_{1}^{e}>0$ and $\hat{a}_{2}^{e}>0$. Since $\Pi\left(a_{1}, a_{2}\right)$ is a concave function, $\left(\hat{a}_{1}^{e}, \hat{a}_{2}^{e}\right)$ is the optimal portfolio. Using the definitions for $m^{+}$and 
$m^{-}$in (A171) and (A172), respectively, we can express the numerator and the denominator in (A169) as

$$
\frac{r^{S}}{r^{N}}\left(c m^{+}-\tau\right)
$$

and

$$
\frac{m^{+}}{m^{-}}-1
$$

resulting, using $\frac{1}{\mu_{1}^{N}} \frac{r^{S}}{r^{N}}=\frac{1}{\mu_{1}^{S}}$, in (74). (75) is obtained in the same way.

Now assume that the numbers of procedures are bounded from above, so that $\hat{a}_{i}^{e} \leq U_{i}$ for $i=1,2$. Note that $\left(a_{1}^{0}, a_{2}^{0}\right)$, given by (74) and (75), still satisfy the first-order conditions (A147) and (A148).

In this case, we aim to solve the following constrained optimization problem:

$$
\begin{array}{ll} 
& \max \Pi\left(a_{1}^{e}, a_{2}^{e}\right) \\
\text { subject to } & 0 \leq a_{1}^{e} \leq U_{1}, \\
& 0 \leq a_{2}^{e} \leq U_{2} .
\end{array}
$$

The corresponding Karush-Kuhn-Tucker (KKT) conditions are as follows:

$$
\begin{aligned}
& \frac{\partial \Pi}{\partial a_{1}^{e}}\left(\hat{a}_{1}^{e}, \hat{a}_{2}^{e}\right)=\mu_{1}, \\
& \frac{\partial \Pi}{\partial a_{2}^{e}}\left(\hat{a}_{1}^{e}, \hat{a}_{2}^{e}\right)=\mu_{2}, \\
& \hat{a}_{1}^{e} \leq U_{1}, \\
& \hat{a}_{2}^{e} \leq U_{2}, \\
& 0 \leq \mu_{1}, \\
& 0 \leq \mu_{2}, \\
& \mu_{1}\left(\hat{a}_{1}^{e}-U_{1}\right)=0, \\
& \mu_{2}\left(\hat{a}_{2}^{e}-U_{2}\right)=0 .
\end{aligned}
$$

Part (1) is obvious with $\mu_{1}=\mu_{2}=0$.

Parts (2) and (3) are similar, so we consider only part (3) here, so that $a_{1}^{0}>U_{1}$ and $a_{2}^{0} \leq U_{2}$. Setting $\hat{a}_{1}^{e}=U_{1}$ satisfies conditions (A180) and (A184). Since $\Pi$ is concave and $U_{1}<a_{1}^{0}, \mu_{1}=$ $\frac{\partial \Pi}{\partial a_{1}^{e}}\left(U_{1}, a_{2}\right)>0$ for all $a_{2}$, which satisfies conditions (A178) and (A182). Let $a_{2}^{0}$ satisfy the first-order condition when $\hat{a}_{1}^{e}=U_{1}$. Hence,

$$
R_{2}=\frac{\pi^{S} \bar{\sigma}_{2}^{S}}{\sqrt{2 \pi}} e^{-\frac{1}{2}\left(Y_{U_{1}}\right)^{2}}+\frac{\pi^{N} \mu_{2}^{N}}{\sqrt{2 \pi}} \int_{X_{U_{1}}}^{+\infty} e^{-\frac{1}{2} v^{2}} d v+\frac{\pi^{S} \mu_{2}^{S}}{\sqrt{2 \pi}} \int_{Y_{U_{1}}}^{+\infty} e^{-\frac{1}{2} v^{2}} d v
$$


where

$$
\begin{aligned}
X_{U_{1}} & =\frac{c-U_{1} \mu_{1}^{N}-\hat{a}_{2}^{0} \mu_{2}^{N}}{U_{1} \bar{\sigma}_{1}^{N}}, \\
Y_{U_{1}} & =\frac{\tau-U_{1} \mu_{1}^{S}-\hat{a}_{2}^{0} \mu_{2}^{S}}{\hat{a}_{2}^{0} \bar{\sigma}_{2}^{S}} .
\end{aligned}
$$

From (A188), we have:

$$
\hat{a}_{2}^{0}=\frac{\tau-U_{1} \mu_{1}^{S}}{\mu_{2}^{S}+\bar{\sigma}_{2}^{S} Y_{U_{1}}}
$$

Plugging (A189) in (A187) and setting $Z_{2}=\left(Y_{U_{1}}\right)^{2}$ we get, with straightforward algebraic manipulation,

$$
X_{U_{1}}=\frac{1}{\bar{\sigma}_{1}^{N}}\left(\frac{c}{U_{1}}-\mu_{1}^{N}-\frac{\mu_{2}^{N}\left(\tau / U_{1}-\mu_{1}^{S}\right)}{\mu_{2}^{S}+\bar{\sigma}_{2}^{S} \sqrt{Z_{2}}}\right)
$$

Dividing all terms in (A186) by $R_{2}$, we use (A190) to obtain equation (79). If $\hat{a}_{2}^{0} \leq U_{2}$, we set $\hat{a}_{2}^{e}=\hat{a}_{2}^{0}$ and $\mu_{2}=0$. Then, conditions (A179), (A181), (A183) and (A185) are satisfied, so that $\left(U_{1}, \hat{a}_{2}^{0}\right)$ is an optimal solution to the constrained problem. If $\hat{a}_{2}^{0}>U_{2}$, we set $\hat{a}_{2}^{e}=U_{2}$ and $\mu_{2}=\frac{\partial \Pi}{\partial a_{2}^{e}}\left(U_{1}, U_{2}\right)$. Since $\Pi$ is concave and $\hat{a}_{2}^{0}>U_{2}, \mu_{2}>0$, which implies that conditions (A179), (A181), (A183) and (A185) hold. Hence, $\left(U_{1}, U_{2}\right)$ is an optimal solution to the constrained problem.

Finally, assume that $a_{1}^{0}>U_{1}$ and $a_{2}^{0}>U_{2}$. When we set $\left(\hat{a}_{1}^{e}, \hat{a}_{2}^{e}\right)=\left(U_{1}, U_{2}\right)$, all KKT conditions are satisfied. Note that conditions (A182) and (A183) are also true since $\Pi$ is concave and $U_{i}<a_{i}^{0}$ for $i=1,2$. 


\section{Appendix B: Modeling Extensions}

\section{B.1. Analysis of Settings with $n>2$ Procedures}

For the analysis of the general setting with $n>2$ types of surgical procedures, it is convenient to introduce the following quantities:

$$
\begin{aligned}
\rho_{i}^{N} & =\frac{\mu_{i}^{N}}{\bar{\sigma}_{i}^{N}}, i=1, \ldots, n, \\
\rho_{i}^{S} & =\frac{\mu_{i}^{S}}{\bar{\sigma}_{i}^{S}}, i=1, \ldots, n, \\
\zeta_{i}^{N} & =\sum_{k \neq i}\left(\rho_{k}^{N}\right)^{2}, i=1, \ldots, n, \\
\zeta_{i}^{S} & =\sum_{k \neq i}\left(\rho_{k}^{S}\right)^{2}, i=1, \ldots, n .
\end{aligned}
$$

The following proposition characterizes the optimal portfolio of elective procedures through the solution to a system of four non-linear equations.

Proposition B1. In the absence of urgent procedures, consider a setting where

$$
\begin{aligned}
\frac{\pi^{N} \mu_{i}^{N}}{2} \operatorname{erfc}\left(\frac{\zeta_{i}^{N}}{\sqrt{2}}\right) & +\frac{\pi^{S} \mu_{i}^{S}}{2} \operatorname{erfc}\left(\frac{\zeta_{i}^{S}}{\sqrt{2}}\right) \leq R_{i} \leq \frac{\pi^{N} \bar{\sigma}_{i}^{N}}{\sqrt{2 \pi}}\left(e^{-\frac{1}{2}\left(\rho_{i}^{N}\right)^{2}}+\rho_{i}^{N} \sqrt{\frac{2}{\pi}} \operatorname{erfc}\left(\frac{\rho_{i}^{N}}{\sqrt{2}}\right)\right) \\
& +\frac{\pi^{S} \bar{\sigma}_{i}^{S}}{\sqrt{2 \pi}}\left(e^{-\frac{1}{2}\left(\rho_{i}^{S}\right)^{2}}+\rho_{i}^{S} \sqrt{\frac{2}{\pi}} \operatorname{erfc}\left(\frac{\rho_{i}^{S}}{\sqrt{2}}\right)\right), i=1, \ldots, n,
\end{aligned}
$$

and let $\left(M_{S}^{*}, \Sigma_{S}^{*}, M_{N}^{*}, \Sigma_{N}^{*}\right)$ be the solution to the system of equations

$$
\begin{aligned}
& M_{S}^{*}=\sum_{i=1}^{n} \mu_{i}^{S}\left(\frac{R_{i}-\mu_{i}^{S}\left(\frac{\pi^{S}}{2} \operatorname{erfc}\left(\frac{\tau-M_{S}^{*}}{\sqrt{2} \Sigma_{S}^{*}}\right)\right)-\mu_{i}^{N}\left(\frac{\pi^{N}}{2} \operatorname{erfc}\left(\frac{c-M_{N}^{*}}{\sqrt{2} \Sigma_{N}^{*}}\right)\right)}{\left(\bar{\sigma}_{i}^{S}\right)^{2}\left(\frac{\pi^{S}}{\sqrt{2 \pi} \Sigma_{S}^{*}} e^{-\frac{1}{2}\left(\frac{\tau-M_{S}^{*}}{\Sigma_{S}^{*}}\right)^{2}}\right)+\left(\bar{\sigma}_{i}^{N}\right)^{2}\left(\frac{\pi^{N}}{\sqrt{2 \pi} \Sigma_{N}^{*}} e^{-\frac{1}{2}\left(\frac{c-M_{N}^{*}}{\Sigma_{N}^{*}}\right)^{2}}\right)}\right), \\
& \Sigma_{S}^{*}=\sqrt{\sum_{i=1}^{n}\left(\sigma_{i}^{S}\right)^{2}\left(\frac{R_{i}-\mu_{i}^{S}\left(\frac{\pi^{S}}{2} \operatorname{erfc}\left(\frac{\tau-M_{S}^{*}}{\sqrt{2} \Sigma_{S}^{*}}\right)\right)-\mu_{i}^{N}\left(\frac{\pi^{N}}{2} \operatorname{erfc}\left(\frac{c-M_{N}^{*}}{\sqrt{2} \Sigma_{N}^{*}}\right)\right)}{\left(\bar{\sigma}_{i}^{S}\right)^{2}\left(\frac{\pi^{S}}{\sqrt{2 \pi} \Sigma_{S}^{*}} e^{-\frac{1}{2}\left(\frac{\tau-M_{S}^{*}}{\Sigma_{S}^{*}}\right)^{2}}\right)+\left(\bar{\sigma}_{i}^{N}\right)^{2}\left(\frac{\pi^{N}}{\sqrt{2 \pi} \Sigma_{N}^{*}} e^{-\frac{1}{2}\left(\frac{c-M_{N}^{*}}{\Sigma_{N}^{*}}\right)^{2}}\right)}\right)^{2}}, \\
& M_{N}^{*}=\sum_{i=1}^{n} \mu_{i}^{N}\left(\frac{R_{i}-\mu_{i}^{S}\left(\frac{\pi^{S}}{2} \operatorname{erfc}\left(\frac{\tau-M_{S}^{*}}{\sqrt{2} \Sigma_{S}^{*}}\right)\right)-\mu_{i}^{N}\left(\frac{\pi^{N}}{2} \operatorname{erfc}\left(\frac{c-M_{N}^{*}}{\sqrt{2} \Sigma_{N}^{*}}\right)\right)}{\left(\bar{\sigma}_{i}^{S}\right)^{2}\left(\frac{\pi^{S}}{\sqrt{2 \pi} \Sigma_{S}^{*}} e^{-\frac{1}{2}\left(\frac{\tau-M_{S}^{*}}{\Sigma_{S}^{*}}\right)^{2}}\right)+\left(\bar{\sigma}_{i}^{N}\right)^{2}\left(\frac{\pi^{N}}{\sqrt{2 \pi} \Sigma_{N}^{*}} e^{-\frac{1}{2}\left(\frac{c-M_{N}^{*}}{\Sigma_{N}^{*}}\right)^{2}}\right)}\right)
\end{aligned}
$$

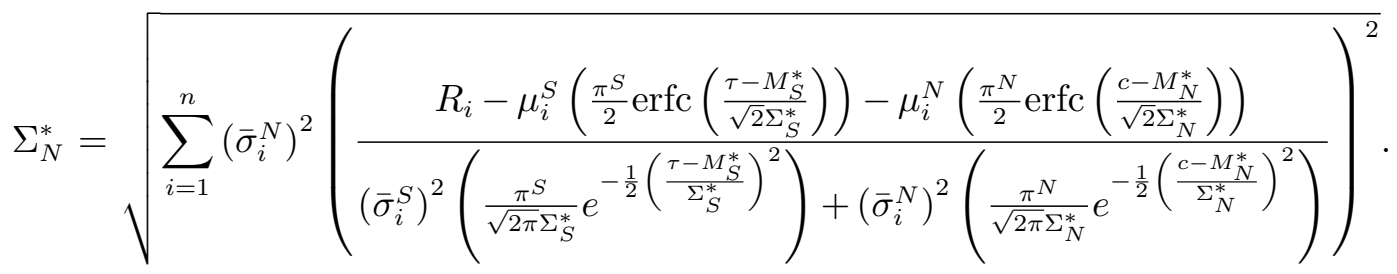


Then, assuming that there is a unique optimal solution, the portfolio of procedures optimizing (29) is given by

$$
\hat{a}_{i}^{e}=\frac{R_{i}-\mu_{i}^{S}\left(\frac{\pi^{S}}{2} \operatorname{erfc}\left(\frac{\tau-M_{S}^{*}}{\sqrt{2} \Sigma_{S}^{*}}\right)\right)-\mu_{i}^{N}\left(\frac{\pi^{N}}{2} \operatorname{erfc}\left(\frac{c-M_{N}^{*}}{\sqrt{2} \Sigma_{N}^{*}}\right)\right)}{\left(\bar{\sigma}_{i}^{S}\right)^{2}\left(\frac{\pi^{S}}{\sqrt{2 \pi} \Sigma_{S}^{*}} e^{-\frac{1}{2}\left(\frac{\tau-M_{S}^{*}}{\Sigma_{S}^{*}}\right)^{2}}\right)+\left(\bar{\sigma}_{i}^{N}\right)^{2}\left(\frac{\pi^{N}}{\sqrt{2 \pi} \Sigma_{N}^{*}} e^{-\frac{1}{2}\left(\frac{c-M_{N}^{*}}{\Sigma_{N}^{*}}\right)^{2}}\right)}, i=1, \ldots, n .
$$

Proposition B1 reduces the problem of allocating the surgery and recovery bed capacity among $n$ procedure types in "elective only" setting to the task of solving a system of four nonlinear equations. For a large number of procedure types, this task is in general much easier to accomplish than directly solving a system of $n$ first-order optimality conditions.

A sharper characterization of the optimal portfolio in "elective only" cases can be obtained in a special setting, where the ratios $\frac{R_{i}}{\mu_{i}^{S}}, \frac{R_{i}}{\mu_{i}^{N}}$, and $\frac{\sigma_{i}^{S}}{\sigma_{i}^{N}}$ are the same for all procedure types $i=1, \ldots, n$. Similar to our discussion of a two-procedure setting above, we will use the term "completely proportional" to denote such a setting. In a completely proportional setting, it is convenient to define

$$
\begin{aligned}
r_{S} & =\frac{R_{i}}{\mu_{i}^{S}}, i=1, \ldots, n, \\
r_{N} & =\frac{R_{i}}{\mu_{i}^{N}}, i=1, \ldots, n, \\
\rho_{\sigma} & =\frac{\bar{\sigma}_{i}^{S}}{\bar{\sigma}_{i}^{N}}, i=1, \ldots, n .
\end{aligned}
$$

Then, the optimal portfolio in a completely proportional setting can be described as follows.

Corollary B1. In a completely proportional setting defined by $\left\{R_{i}, \bar{\sigma}_{i}^{S}\right\}, i=1, \ldots, n$ and $\left\{r_{S}, r_{N}, \rho_{\sigma}\right\}$, let $Z_{P}$ be the unique solution to

$$
\begin{aligned}
\omega_{S} & =\frac{\pi^{S}}{\sqrt{2 \pi}}\left(e^{-\frac{1}{2}\left(\frac{\tau}{\omega_{S} Z_{P}}-\rho_{P}^{S}\right)^{2}}+\sqrt{\frac{\pi}{2}} \rho_{P}^{S} \operatorname{erfc}\left(\frac{1}{\sqrt{2}}\left(\frac{\tau}{\omega_{S} Z_{P}}-\rho_{P}^{S}\right)\right)\right) \\
& +\frac{\pi^{N}}{\sqrt{2 \pi} \rho_{\sigma}}\left(e^{-\frac{1}{2}\left(\frac{c \rho_{\sigma}}{\omega_{S} Z_{P}}-\rho_{P}^{N}\right)^{2}}+\sqrt{\frac{\pi}{2}} \rho_{P}^{N} \operatorname{erfc}\left(\frac{1}{\sqrt{2}}\left(\frac{c \rho_{\sigma}}{\omega_{S} Z_{P}}-\rho_{P}^{N}\right)\right)\right),
\end{aligned}
$$

where

$$
\begin{aligned}
\omega_{S} & =\sqrt{\sum_{i=1}^{n}\left(\frac{R_{i}}{\bar{\sigma}_{i}^{S}}\right)^{2}}, \\
\rho_{P}^{S} & =\frac{\omega_{S}}{r_{S}} \\
\rho_{P}^{N} & =\frac{\rho_{\sigma} \omega_{S}}{r_{N}} .
\end{aligned}
$$

Then, the optimal portfolio of procedures is given by

$$
\hat{a}_{i}^{e}=\frac{R_{i}}{\left(\bar{\sigma}_{i}^{S}\right)^{2}} Z_{P}, i=1, \ldots, n .
$$



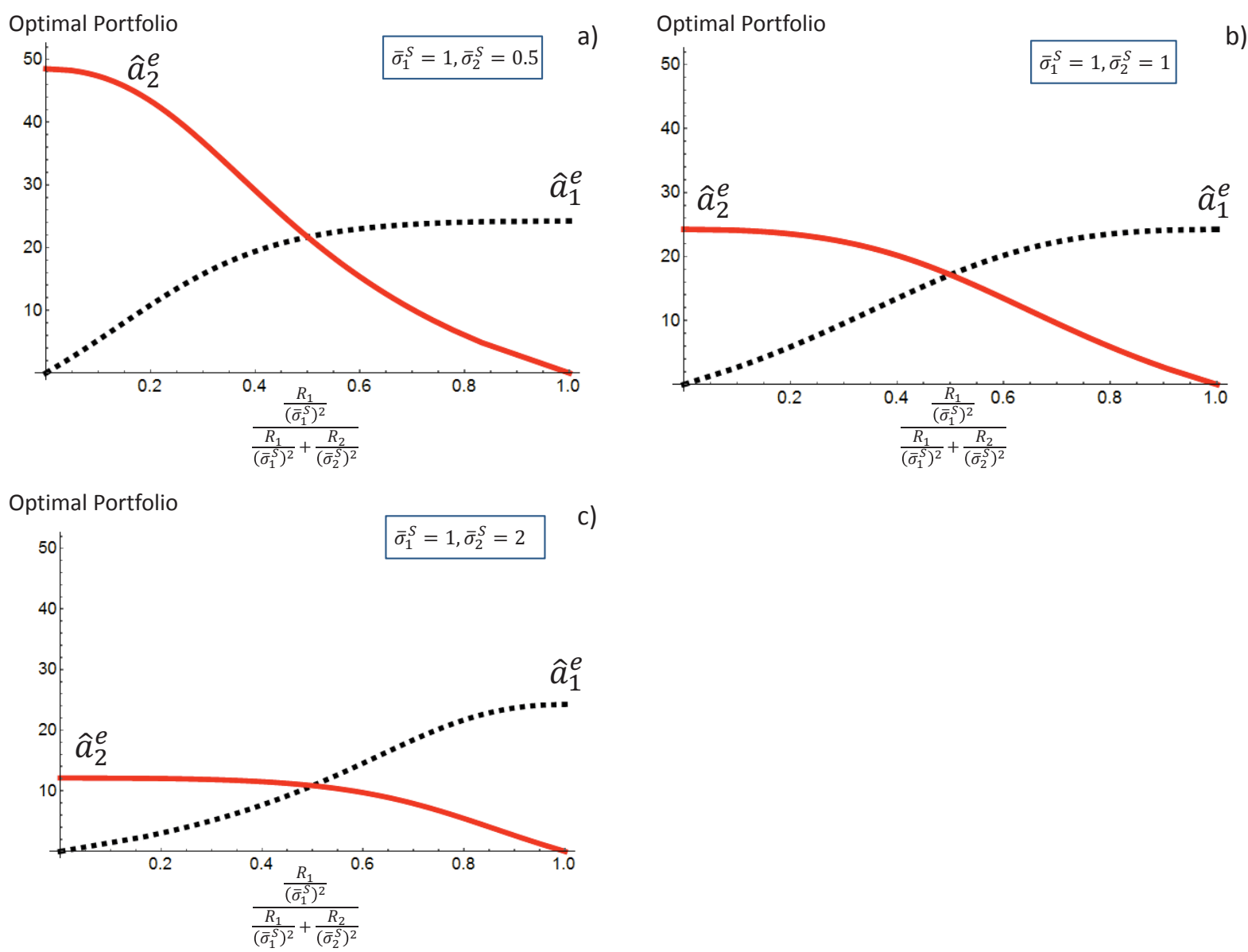

Figure B1: The optimal portfolio in the case of $n=2$ procedures in the completely proportional setting as a function of revenue values for different values of standard deviation parameters $\bar{\sigma}_{1}^{S}$ and $\bar{\sigma}_{2}^{S}$ $\left(\left(\frac{R_{1}}{\bar{\sigma}_{1}^{S}}\right)^{2}+\left(\frac{R_{2}}{\bar{\sigma}_{2}^{S}}\right)^{2}=1, \tau=50, c=100, \pi^{N}=\pi^{S}=1.5, r_{N}=r_{S}=1, \rho_{\sigma}=0.5\right)$.

As Corollary B1 states, in a completely proportional setting, the overall load that a portfolio of procedures exerts on the surgical and recovery bed capacity can be described via a common factor $Z_{P}$ that plays a role of an effective portfolio size.

Figure B1 illustrates the results of Corollary B1 in the case of two procedure types. In this figure we show how the composition of the optimal portfolio is affected by the revenues and standard deviation parameters associated with procedures. In particular, for each combination of $\bar{\sigma}_{1}^{S}$ and $\bar{\sigma}_{2}^{S}$, we change the procedure revenues so as to keep the parameter $\omega_{S}$ constant. Thus, the value of $Z_{P}$ also remains constant and the optimal portfolio composition, as stated in (B18), is determined by the ratios $\frac{R_{1}}{\left(\bar{\sigma}_{1}^{S}\right)^{2}}$ and $\frac{R_{2}}{\left(\bar{\sigma}_{2}^{S}\right)^{2}}$. Note that, as (B18) implies, the value of the total daily revenue generated by the optimal portfolio, $R_{1} \hat{a}_{1}^{e}+R_{2} \hat{a}_{2}^{e}=\left(\left(\frac{R_{1}}{\bar{\sigma}_{1}^{S}}\right)^{2}+\left(\frac{R_{2}}{\bar{\sigma}_{2}^{S}}\right)^{2}\right) Z_{P}=Z_{P}$ remains constant in each graph. As Figure B1 indicates, the contribution of a particular procedure to the optimal way to generate a fixed amount of total daily revenue grows with the revenue contribution of that 
procedure, as expected. Note that in the completely proportional setting, an increase in the value of $\bar{\sigma}_{2}^{S}$ for constant $\rho^{\sigma}$ implies a corresponding increase in $\bar{\sigma}_{2}^{N}$, and, consequently, results in a reduced "attractiveness" of type-2 procedures. This effect is illustrated by the reduction in the number of type-2 procedures included in the optimal portfolio as $\bar{\sigma}_{2}^{S}$ increases from 0.5 (Figure B1a) to 2 (Figure B1c).

The results of Proposition B1 and Corollary B1 provide important analytical insights on how two-dimensional hospital service capacity should be allocated among an arbitrary number of procedure types in special settings. In the general setting, however, in order to describe the optimal composition of the portfolio, it is necessary to resort to a numerical study.

\section{Proof of Proposition B1}

We start by defining, for a given portfolio of elective procedures $\left(a_{1}^{e}, \ldots, a_{n}^{e}\right)$,

$$
\begin{aligned}
M_{S} & =\sum_{i=1}^{n} a_{i}^{e} \mu_{i}^{S}, \\
\left(\Sigma_{S}\right)^{2} & =\sum_{i=1}^{n}\left(a_{i}^{e}\right)^{2}\left(\bar{\sigma}_{i}^{S}\right)^{2}, \\
M_{N} & =\sum_{i=1}^{n} a_{i}^{e} \mu_{i}^{N}, \\
\left(\Sigma_{N}\right)^{2} & =\sum_{i=1}^{n}\left(a_{i}^{e}\right)^{2}\left(\bar{\sigma}_{i}^{N}\right)^{2},
\end{aligned}
$$

and calculating the expected value of the overtime in surgery durations on day $t$ :

$$
\begin{aligned}
& E\left[\left(D_{t}\left(a_{1}^{e}, \ldots, a_{n}^{e}\right)-\tau\right)^{+}\right]=\frac{1}{\sqrt{2 \pi \sum_{i=1}^{n}\left(a_{i}^{e}\right)^{2}\left(\bar{\sigma}_{i}^{S}\right)^{2}}} \int_{\tau}^{\infty}(x-\tau) e^{-\frac{\left(x-\sum_{i=1}^{n} a_{i}^{e} \mu_{i}^{S}\right)^{2}}{2\left(\sum_{i=1}^{n}\left(a_{i}^{e}\right)^{2}\left(\bar{\sigma}_{i}^{S}\right)^{2}\right)}} d x \\
& =\frac{1}{\sqrt{2 \pi \Sigma_{S}^{2}}} \int_{\tau}^{\infty}(x-\tau) e^{-\frac{\left(x-M_{S}\right)^{2}}{2 \Sigma_{S}^{2}}} d x \\
& =\frac{1}{\sqrt{2 \pi \Sigma_{S}^{2}}} \int_{0}^{\infty} y e^{-\frac{\left(y+\tau-M_{S}\right)^{2}}{2 \Sigma_{S}^{2}}} d y=\frac{\Sigma_{S}}{\sqrt{2 \pi}} \int_{0}^{\infty} z e^{-\frac{1}{2}\left(z+\frac{\tau-M_{S}}{\Sigma_{S}}\right)^{2}} d z \\
& =\frac{\Sigma_{S}}{\sqrt{2 \pi}}\left(\int_{\frac{\tau-M_{S}}{\Sigma_{S}}}^{\infty} v e^{-\frac{1}{2} v^{2}} d v-\left(\frac{\tau-M_{S}}{\Sigma_{S}}\right) \int_{\frac{\tau-M_{S}}{\Sigma_{S}}}^{\infty} e^{-\frac{1}{2} v^{2}} d v\right) \\
& =\frac{\Sigma_{S}}{\sqrt{2 \pi}}\left(e^{-\frac{1}{2}\left(\frac{\tau-M_{S}}{\Sigma_{S}}\right)^{2}}-\left(\frac{\tau-M_{S}}{\Sigma_{S}}\right) \int_{\frac{\tau-M_{S}}{\Sigma_{S}}}^{\infty} e^{-\frac{1}{2} v^{2}} d v\right) .
\end{aligned}
$$

In a similar fashion, for the "overflow" in the number of occupied beds, we have

$$
E\left[\left(O_{t}\left(a_{1}^{e}, \ldots, a_{n}^{e}\right)-c\right)^{+}\right]=\frac{\Sigma_{N}}{\sqrt{2 \pi}}\left(e^{-\frac{1}{2}\left(\frac{c-M_{N}}{\Sigma_{N}}\right)^{2}}-\left(\frac{c-M_{N}}{\Sigma_{N}}\right) \int_{\frac{c-M_{N}}{\Sigma_{N}}}^{\infty} e^{-\frac{1}{2} v^{2}} d v\right) .
$$


Thus, the hospital profit can be expressed as

$$
\begin{aligned}
\Pi\left(a_{1}^{e}, \ldots, a_{n}^{e}\right) & =\sum_{i=1}^{n} R_{i} a_{i}^{e}-\frac{\pi^{S} \Sigma_{S}}{\sqrt{2 \pi}}\left(e^{-\frac{1}{2}\left(\frac{\tau-M_{S}}{\Sigma_{S}}\right)^{2}}-\left(\frac{\tau-M_{S}}{\Sigma_{S}}\right) \int_{\frac{\tau-M_{S}}{\Sigma_{S}}}^{\infty} e^{-\frac{1}{2} v^{2}} d v\right) \\
& -\frac{\pi^{N} \Sigma_{N}}{\sqrt{2 \pi}}\left(e^{-\frac{1}{2}\left(\frac{c-M_{N}}{\Sigma_{N}}\right)^{2}}-\left(\frac{c-M_{N}}{\Sigma_{N}}\right) \int_{\frac{c-M_{N}}{\Sigma_{N}}}^{\infty} e^{-\frac{1}{2} v^{2}} d v\right) .
\end{aligned}
$$

Calculating the partial derivative of the objective function with respect to $a_{i}^{e}, i=1, \ldots, n$, we have,

$$
\begin{aligned}
& \frac{\partial \Pi}{\partial a_{i}^{e}} \\
= & R_{i}-\frac{\pi^{N}}{\sqrt{2 \pi}}\left(\frac{\left(\bar{\sigma}_{i}^{N}\right)^{2} a_{i}^{e}}{\Sigma_{N}}\left(e^{-\frac{1}{2}\left(\frac{c-M_{N}}{\Sigma_{N}}\right)^{2}}-\left(\frac{c-M_{N}}{\Sigma_{N}}\right) \int_{\frac{c-M_{N}}{\Sigma_{N}}}^{\infty} e^{-\frac{1}{2} v^{2}} d v\right)+\Sigma_{N}\left(-\int_{\frac{c-M_{N}}{\Sigma_{N}}}^{\infty} e^{-\frac{1}{2} v^{2}} d v\right) \frac{\partial\left(\frac{c-M_{N}}{\Sigma_{N}}\right)}{\partial a_{i}^{e}}\right) \\
- & \frac{\pi^{S}}{\sqrt{2 \pi}}\left(\frac{\left(\bar{\sigma}_{i}^{S}\right)^{2} a_{i}^{e}}{\Sigma_{S}}\left(e^{-\frac{1}{2}\left(\frac{\tau-M_{S}}{\Sigma_{S}}\right)^{2}}-\left(\frac{\tau-M_{S}}{\Sigma_{S}}\right) \int_{\frac{\tau-M_{S}}{\Sigma_{S}}}^{\infty} e^{-\frac{1}{2} v^{2}} d v\right)+\Sigma_{S}\left(-\int_{\frac{\tau-M_{S}}{\Sigma_{S}}}^{\infty} e^{-\frac{1}{2} v^{2}} d v\right) \frac{\partial\left(\frac{\tau-M_{S}}{\Sigma_{S}}\right)}{\partial a_{i}^{e}}\right) .
\end{aligned}
$$

Note that

$$
\begin{aligned}
& \Sigma_{S} \frac{\partial\left(\frac{\tau-M_{S}}{\Sigma_{S}}\right)}{\partial a_{i}^{e}}=\Sigma_{S}\left(\frac{-\mu_{i}^{S} \Sigma_{S}-\left(\tau-M_{S}\right) \frac{\left(\bar{\sigma}_{i}^{S}\right)^{2} a_{i}^{e}}{\Sigma_{S}}}{\Sigma_{S}^{2}}\right) \\
= & -\mu_{i}^{S}-\left(\frac{\tau-M_{S}}{\Sigma_{S}}\right)\left(\frac{\left(\bar{\sigma}_{i}^{S}\right)^{2} a_{i}^{e}}{\Sigma_{S}}\right),
\end{aligned}
$$

and

$$
\Sigma_{N} \frac{\partial\left(\frac{c-M_{N}}{\Sigma_{N}}\right)}{\partial a_{i}^{e}}=-\mu_{i}^{N}-\left(\frac{c-M_{N}}{\Sigma_{N}}\right)\left(\frac{\left(\bar{\sigma}_{i}^{N}\right)^{2} a_{i}^{e}}{\Sigma_{N}}\right) .
$$

Then, (B26) becomes

$$
\begin{aligned}
\frac{\partial \Pi}{\partial a_{i}} & =R_{i}-\frac{\pi^{N}}{\sqrt{2 \pi}}\left(\frac{\left(\bar{\sigma}_{i}^{N}\right)^{2} a_{i}^{e}}{\Sigma_{N}}\left(e^{-\frac{1}{2}\left(\frac{c-M_{N}}{\Sigma_{N}}\right)^{2}}\right)+\mu_{i}^{N} \int_{\frac{c-M_{N}}{\Sigma_{N}}}^{\infty} e^{-\frac{1}{2} v^{2}} d v\right) \\
& -\frac{\pi^{S}}{\sqrt{2 \pi}}\left(\frac{\left(\bar{\sigma}_{i}^{S}\right)^{2} a_{i}^{e}}{\Sigma_{S}}\left(e^{\left.-\frac{1}{2}\left(\frac{\tau-M_{S}}{\Sigma_{S}}\right)^{2}\right)+\mu_{i}^{S}} \int_{\frac{\tau-M_{S}}{\Sigma_{S}}}^{\infty} e^{-\frac{1}{2} v^{2}} d v\right)\right. \\
& =R_{i}-\frac{\pi^{N}}{\sqrt{2 \pi}}\left(\frac{\left(\bar{\sigma}_{i}^{N}\right)^{2} a_{i}^{e}}{\Sigma_{N}}\left(e^{-\frac{1}{2}\left(\frac{c-M_{N}}{\Sigma_{N}}\right)^{2}}\right)+\sqrt{\frac{\pi}{2}} \mu_{i}^{N} \operatorname{erfc}\left(\frac{c-M_{N}}{\sqrt{2} \Sigma_{N}}\right)\right) \\
& -\frac{\pi^{S}}{\sqrt{2 \pi}}\left(\frac{\left(\bar{\sigma}_{i}^{S}\right)^{2} a_{i}^{e}}{\Sigma_{S}}\left(e^{-\frac{1}{2}\left(\frac{\tau-M_{S}}{\Sigma_{S}}\right)^{2}}\right)+\sqrt{\frac{\pi}{2}} \mu_{i}^{S} \operatorname{erfc}\left(\frac{\tau-M_{S}}{\sqrt{2} \Sigma_{S}}\right)\right) .
\end{aligned}
$$

Note that (B5) ensures that $\lim _{a_{i}^{e} \rightarrow 0} \frac{\partial \Pi}{\partial a_{i}^{e}} \geq 0$ and $\lim _{a_{i}^{e} \rightarrow \infty} \frac{\partial \Pi}{\partial a_{i}^{e}} \leq 0$ for any $a_{j}^{e} \geq 0, j \neq i$. 
Next, equating (B29) to 0 , we get

$$
a_{i}^{e}=\frac{R_{i}-\mu_{i}^{S}\left(\frac{\pi^{S}}{2} \operatorname{erfc}\left(\frac{\tau-M_{S}}{\sqrt{2} \Sigma_{S}}\right)\right)-\mu_{i}^{N}\left(\frac{\pi^{N}}{2} \operatorname{erfc}\left(\frac{c-M_{N}}{\sqrt{2} \Sigma_{N}}\right)\right)}{\left(\bar{\sigma}_{i}^{S}\right)^{2}\left(\frac{\pi^{S}}{\sqrt{2 \pi} \Sigma_{S}} e^{-\frac{1}{2}\left(\frac{\tau-M_{S}}{\Sigma_{S}}\right)^{2}}\right)+\left(\bar{\sigma}_{i}^{N}\right)^{2}\left(\frac{\pi^{N}}{\sqrt{2 \pi} \Sigma_{N}} e^{-\frac{1}{2}\left(\frac{c-M_{N}}{\Sigma_{N}}\right)^{2}}\right)} .
$$

Substituting (B30) into

$$
\begin{aligned}
M_{S} & =\sum_{i=1}^{n} a_{i}^{e} \mu_{i}^{S}, \\
\Sigma_{S} & =\sqrt{\sum_{i=1}^{n}\left(a_{i}^{e}\right)^{2}\left(\sigma_{i}^{S}\right)^{2}}, \\
M_{N} & =\sum_{i=1}^{n} a_{i}^{e} \mu_{i}^{N}, \\
\Sigma_{N} & =\sqrt{\sum_{i=1}^{n}\left(a_{i}^{e}\right)^{2}\left(\Sigma^{N}\left(\mathbf{a}^{e}, \mathbf{\Xi}_{t}\right)_{i}\right)^{2}},
\end{aligned}
$$

we obtain (B6)-(B9) as a set of equations that $M_{S}, \Sigma_{S}, M_{L}$, and $\Sigma_{L}$ must satisfy. Denoting the solution to this set of equations as $M_{S}^{*}, \Sigma_{S}^{*}, M_{L}^{*}$, and $\Sigma_{L}^{*}$, we obtain (B10).

\section{Proof of Corollary B1}

In a completely proportional setting, (B10) becomes

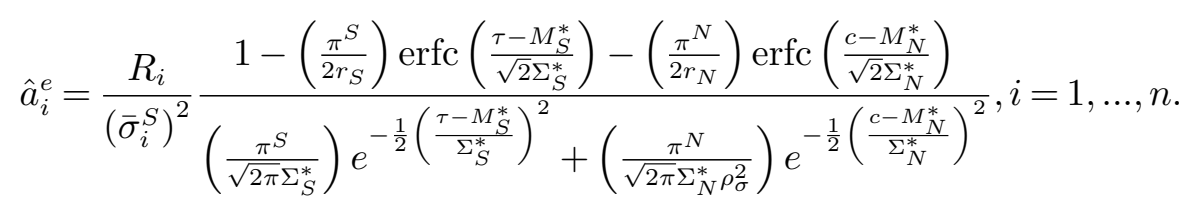

Let us designate

$$
Z_{P}=\frac{1-\left(\frac{\pi^{S}}{2 r_{S}}\right) \operatorname{erfc}\left(\frac{\tau-M_{S}^{*}}{\sqrt{2} \Sigma_{S}^{*}}\right)-\left(\frac{\pi^{N}}{2 r_{N}}\right) \operatorname{erfc}\left(\frac{c-M_{N}^{*}}{\sqrt{2} \Sigma_{N}^{*}}\right)}{\left(\frac{\pi^{S}}{\sqrt{2 \pi} \Sigma_{S}^{*}}\right) e^{-\frac{1}{2}\left(\frac{\tau-M_{S}^{*}}{\Sigma_{S}^{*}}\right)^{2}}+\left(\frac{\pi^{N}}{\sqrt{2 \pi} \Sigma_{N}^{*} \rho_{\sigma}^{2}}\right) e^{-\frac{1}{2}\left(\frac{c-M_{N}^{*}}{\Sigma_{N}^{*}}\right)^{2}}}
$$

and note that

$$
M_{S}^{*}=\sum_{i=1}^{n} \mu_{i}^{S} \hat{a}_{i}^{e}=Z_{P} \sum_{i=1}^{n} \mu_{i}^{S} \frac{R_{i}}{\left(\bar{\sigma}_{i}^{S}\right)^{2}}=\frac{Z_{P}}{r_{S}} \sum_{i=1}^{n}\left(\frac{R_{i}}{\bar{\sigma}_{i}^{S}}\right)^{2}=\frac{Z_{P}}{r_{S}} \omega_{S}^{2}
$$

with

$$
\omega_{S}=\sqrt{\sum_{i=1}^{n}\left(\frac{R_{i}}{\bar{\sigma}_{i}^{S}}\right)^{2}}
$$

and

$$
M_{N}^{*}=\frac{Z_{P}}{r_{N}} \omega_{S}^{2}
$$




$$
\begin{aligned}
\Sigma_{S}^{*} & =Z_{P} \omega_{S}, \\
\Sigma_{N}^{*} & =\frac{Z_{P}}{\rho_{\sigma}} \omega_{S} .
\end{aligned}
$$

Then, (B36) can be expressed as

$$
Z_{P}=\frac{1-\left(\frac{\pi^{S}}{2 r_{S}}\right) \operatorname{erfc}\left(\frac{\tau}{\sqrt{2} \omega_{S} Z_{P}}-\frac{\omega_{S}}{\sqrt{2} r_{S}}\right)-\left(\frac{\pi^{N}}{2 r_{N}}\right) \operatorname{erfc}\left(\frac{c \rho_{\sigma}}{\sqrt{2} \omega_{S} Z_{P}}-\frac{\rho_{\sigma} \omega_{S}}{\sqrt{2} r_{N}}\right)}{\left(\frac{\pi^{S}}{\sqrt{2 \pi} \omega_{S} Z_{P}}\right) e^{-\frac{1}{2}\left(\frac{\tau}{\omega_{S} Z_{P}}-\frac{\omega_{S}}{r_{S}}\right)^{2}}+\left(\frac{\pi^{N}}{\sqrt{2 \pi} \omega_{S} Z_{P} \rho_{\sigma}}\right) e^{-\frac{1}{2}\left(\frac{c \rho_{\sigma}}{\omega_{S} Z_{P}}-\frac{\rho_{\sigma} \omega_{S}}{r_{N}}\right)^{2}}}
$$

which is the same as (B14). Note that the right-hand side of (B42) is a decreasing function of $Z_{P}$, so that (B42) has a unique solution.

\section{B.2. Special case for single procedure type: Comparing the exact and the CLT-based solutions}

In the analysis presented in Section 4, the key role is played by the CLT-based approximations to the required amounts of front-end and back-room resources derived in Proposition 1. In order to investigate the quality of this approximation, we consider a special case where the distributions of the surgical times and patient LOS durations allow for closed-form expressions of the optimal number of elective procedures without having to utilize the CLT limits.

We assume that the daily number of urgent procedures is deterministic and equal to $a^{u}$, and there is only one surgical team, so that the surgical and LOS duration pairs for all procedures performed on a given day are perfectly correlated. Since the approximations of Proposition 1 rely on the limit where the number of surgical teams goes to infinity, a setting with a single surgical team can provide a "worst-case" comparison with the CLT-based results.

In the special case we consider, we assume that the surgical duration $S$ and LOS $L$ for a procedure performed on any day $t$ can each take one of the two values, so that the joint distribution for $(S, L)$ pairs can be expressed as

$$
\begin{aligned}
& p\left(S=s_{1}, L=\ell_{1}\right)=p q, \\
& p\left(S=s_{2}, L=\ell_{1}\right)=(1-p) q, \\
& p\left(S=s_{1}, L=\ell_{2}\right)=p(1-q), \\
& p\left(S=s_{2}, L=\ell_{2}\right)=(1-p)(1-q),
\end{aligned}
$$

where we have dropped the dependence on day index $t$ and procedure number $j=1, \ldots, a_{e}+a_{u}$. The corresponding marginal distributions for the surgical and LOS durations are:

$$
P\left(S=s_{1}\right)=p, P\left(S=s_{2}\right)=1-p
$$




$$
P\left(L=\ell_{1}\right)=q, \quad P\left(L=\ell_{2}\right)=1-q,
$$

with $s_{1}<s_{2}$ and $\ell_{1}<\ell_{2}$. In (B43) we implicitly assume that the surgical and LOS durations are not correlated, but our analysis will also apply to any value of the correlation coefficient between $L$ and $S$ which results in the same marginal distributions (B44). For the analysis below, it is convenient to define

$$
\begin{aligned}
& r_{S}=\frac{R}{p s_{1}+(1-p) s_{2}}, \\
& r_{N}=\frac{R}{q \ell_{1}+(1-q) \ell_{2}},
\end{aligned}
$$

and

$$
\Phi_{B}(v)= \begin{cases}1, & \text { if } v<0, \\
\sum_{y=v}^{\ell_{2}-\ell_{1}}\left(\begin{array}{c}
\ell_{2}-\ell_{1} \\
y
\end{array}\right)(1-q)^{y-\ell_{1}} q^{\ell_{2}-\ell_{1}-y}\left(\frac{\ell_{1}+y}{q \ell_{1}+(1-q) \ell_{2}}\right), & \text { if } 0 \leq v \leq \ell_{2}-\ell_{1}, \\
0, & \text { if } v>\ell_{2}-\ell_{1} .\end{cases}
$$

In addition, we define the set of values

$$
I_{a}=\left\{\frac{\tau}{s_{2}}, \frac{\tau}{s_{1}}, \frac{c}{\ell_{2}}, \ldots, \frac{c}{\ell_{1}}\right\}
$$

The following result describes the number of elective procedures, $\hat{a}^{e}$ that maximizes the hospital's expected daily profit $\Pi\left(a^{e}\right)$ subject to $a_{e} \geq 0$ in the presence of a fixed daily number of urgent procedures. To exclude unrealistic scenarios from our analysis, we assume that the hospital has enough capacity to handle all urgent procedures for any realization of the surgery durations and patient LOS, i.e., that

$$
\begin{aligned}
& a^{u} s_{2}<\tau, \\
& a^{u} \ell_{2}<c .
\end{aligned}
$$

While the assumptions (B49)-(B50) simplify the algebraic analysis, they are non-essential, as our results can be easily extended to the settings that do not satisfy these conditions.

Proposition B2. Consider a setting with a single procedure type described by (B43) and a fixed daily number of urgent patients $a^{u}$. Suppose that (B49)-(B50) hold and that, in addition, the set (B48) contains $\ell_{2}-\ell_{1}+3$ distinct elements, and

$$
\begin{aligned}
& \left(\frac{\pi^{S}}{r_{S}}\right)\left(\left(\frac{(1-p) s_{2}}{p s_{1}+(1-p) s_{2}}\right) \eta\left(a s_{2}-\tau\right)+\left(\frac{p s_{1}}{p s_{1}+(1-p) s_{2}}\right) \eta\left(a s_{1}-\tau\right)\right) \\
+ & \left(\frac{\pi^{N}}{r_{N}}\right) \Phi_{B}\left(\left[\frac{c}{a}-\ell_{1}\right]+1\right) \neq 1, \quad \forall a \in I_{a},
\end{aligned}
$$

where

$$
\eta(x)= \begin{cases}1, & \text { if } x \geq 0 \\ 0, & \text { if } x<0\end{cases}
$$


and $[x]$ is the integer part of $x$.

Then, the following statements hold.

a) The expected daily profit $\Pi\left(a^{e}\right)$ is a concave function of $a^{e}$ with a unique maximum.

b) If $\frac{\pi^{S}}{r_{S}}+\frac{\pi^{N}}{r_{N}}<1$, the optimal number of elective procedures is given by $a^{e}=U$.

c) For

$$
\frac{\pi^{S}}{r_{S}}+\frac{\pi^{N}}{r_{N}}>1
$$

define the following sets

$$
\begin{aligned}
\Delta_{\tau, i}= & \left(\left(\pi^{S}, \pi^{N}\right) \mid\left(\frac{\pi^{N}}{r_{N}}\right) \Phi_{B}\left(\ell_{2}-\ell_{1}+1-\left[\left(\ell_{2}-\ell_{1}+1-\left(\left(\frac{c}{\tau}\right) s_{i}-\ell_{1}\right)^{+}\right)\right]\right)<1\right. \\
< & \left.\frac{\pi^{S}}{r_{S}}\left(\frac{(1-p) s_{2}}{p s_{1}+(1-p) s_{2}}\right)+\left(\frac{\pi^{N}}{r_{N}}\right) \Phi_{B}\left(\ell_{2}-\ell_{1}+1-\left[\left(\ell_{2}-\ell_{1}+1-\left(\left(\frac{c}{\tau}\right) s_{i}-\ell_{1}\right)^{+}\right)\right]\right)\right), \\
& i=1,2,
\end{aligned}
$$

and

$$
\begin{aligned}
\Delta_{c, m}=\left\{\begin{array}{l}
\left(\left(\pi^{S}, \pi^{N}\right) \mid\left(\frac{\pi^{N}}{r_{N}}\right) \Phi_{B}\left(\ell_{2}-\ell_{1}+1-m\right)<1<\left(\frac{\pi^{N}}{r_{N}}\right) \Phi_{B}\left(\ell_{2}-\ell_{1}-m\right)\right), \text { if } m<\ell_{2}-\left(\frac{c}{\tau}\right) s_{2}, \\
\left(\pi^{S}, \pi^{N}\right) \mid \frac{\pi^{S}}{r_{S}}\left(\frac{(1-p) s_{2}}{p s_{1}+(1-p) s_{2}}\right)+\left(\frac{\pi^{N}}{r_{N}}\right) \Phi_{B}\left(\ell_{2}-\ell_{1}+1-m\right)<1 \\
\left.<\frac{\pi^{S}}{r_{S}}\left(\frac{(1-p) s_{2}}{p s_{1}+(1-p) s_{2}}\right)+\left(\frac{\pi^{N}}{r_{N}}\right) \Phi_{B}\left(\ell_{2}-\ell_{1}-m\right)\right), \text { if } \ell_{2}-\left(\frac{c}{\tau}\right) s_{2}<m<\ell_{2}-\left(\frac{c}{\tau}\right) s_{1}, \\
\left(\left(\pi^{S}, \pi^{N}\right) \mid\left(\frac{\pi^{N}}{r_{N}}\right) \Phi_{B}\left(\ell_{2}-\ell_{1}+1-m\right)<1<\left(\frac{\pi^{N}}{r_{N}}\right) \Phi_{B}\left(\ell_{2}-\ell_{1}-m\right)\right), \text { if } m>\ell_{2}-\left(\frac{c}{\tau}\right) s_{1},
\end{array}\right. \\
m=0, \ldots, \ell_{2}-\ell_{1},
\end{aligned}
$$

and let

$$
\hat{a}= \begin{cases}\frac{\tau}{s_{i}}, & \text { if }\left(\pi^{S}, \pi^{N}\right) \in \Delta_{\tau, i}, i=1,2, \\ \frac{c}{\ell_{2}-m}, & \text { if }\left(\pi^{S}, \pi^{N}\right) \in \Delta_{c, m}, m=0, \ldots, \ell_{2}-\ell_{1} .\end{cases}
$$

Then, the optimal number of elective procedures is given by:

$$
\hat{a}^{e}=\min \left\{\hat{a}-a^{u}, U\right\} .
$$

Figure B2 illustrates the effect of using the CLT-based approximation on the optimal numbers of elective procedures in the setting without urgent procedures. In this Figure, we use the symmetric two-value distributions for the surgical durations and the patient LOS, and, similar to Figure 2, we vary the bed capacity factor as well as the relative composition of the set of penalty costs: "backroom" penalty cost only (Figure B2a), "balanced" penalty costs (Figure B2b), and "front-end" penalty cost only (Figure B2c). As Figure B2 indicates, the CLT-based approximation provides an adequate representation of the exact values in settings where the "back room" capacity is limited and its overload is costly. On the other hand, when bed capacity is substantial or extending it is 


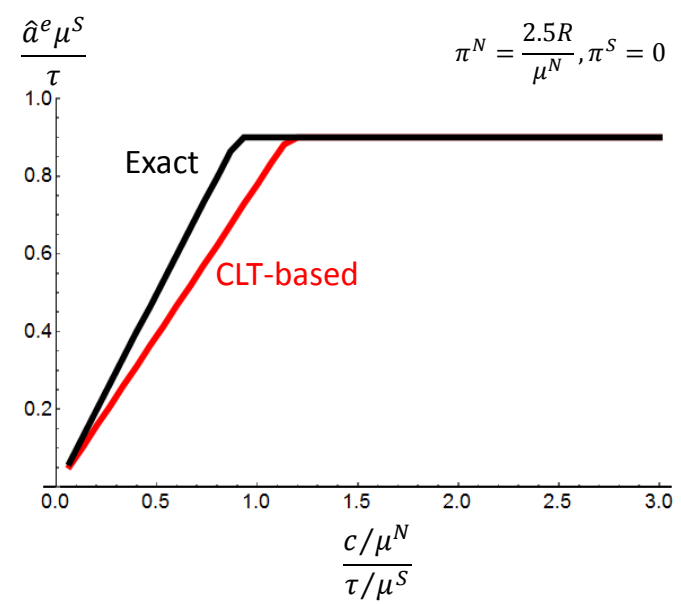

(a)

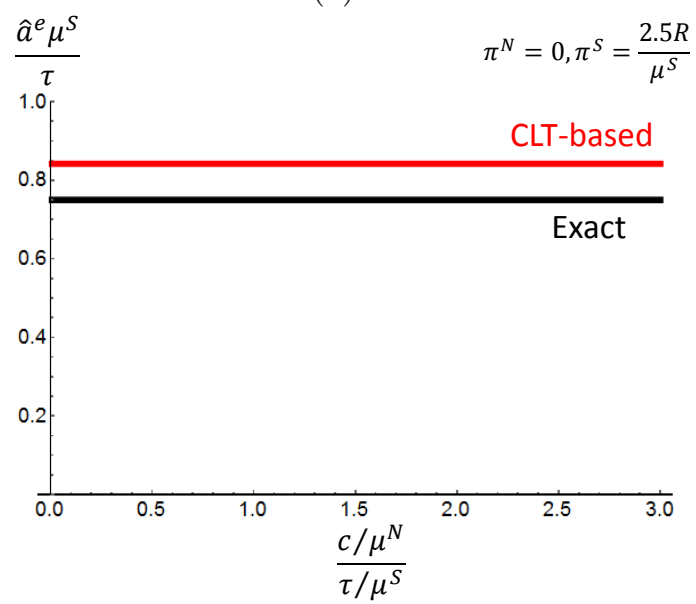

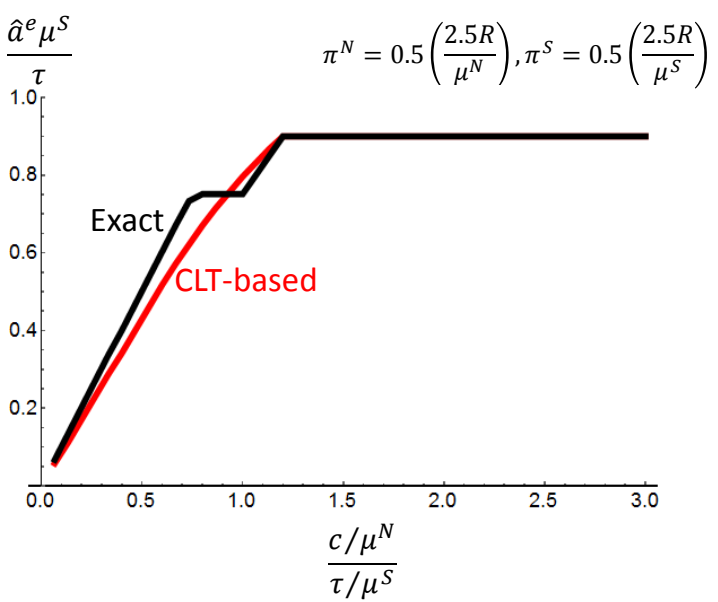

(b)

(c)

Figure B2 : The exact optimal and the CLT-based expected daily surgical loads from elective procedures, $\frac{\hat{a}^{e} \mu^{S}}{\tau}$ in the absence of urgent procedures, as a function of the bed capacity factor $\frac{\frac{c}{\mu^{N}}}{\frac{\tau}{\mu^{S}}}$ for three different penalty cost settings: (a) $\pi^{S}=\frac{2.5 R}{\mu^{S}}, \pi^{N}=0$, (b) $\pi^{S}=0.5\left(\frac{2.5 R}{\mu^{S}}\right), \pi^{N}=0.5\left(\frac{2.5 R}{\mu^{N}}\right)$, and (c) $\pi^{S}=0, \pi^{N}=\frac{2.5 R}{\mu^{N}}$ $\left(\mu_{S}=\mu_{N}=3, s_{1}=\mu_{S}-1, s_{2}=\mu_{S}+1, \ell_{1}=\mu_{N}-1, \ell_{2}=\mu_{N}+1, p=q=0.5, R=1, \tau=15, U=0.9 \frac{\tau}{\mu_{S}}\right)$.

not financially taxing, the optimal management of the "front-end" capacity takes precedence, and the quality of the CLT-based approximation decreases. While the CLT-based values for the daily numbers of elective procedures do not systematically "overshoot" or "undershoot" the exact ones, it is clear that the normal approximation is less successful in representing the two-valued surgery durations than it is in modeling binomial distribution of the number of required beds. Our results indicate that the CLT-based approach combines the clear benefit of being able to handle arbitrary surgery and LOS distributions with adequate performance in settings where a proper accounting must be made for the presence of a limited "backroom" capacity. However, the overall assessment of the value of the CLT-based approach must also weigh in potential costs associated with using the approximation in settings where the "front-end" capacity becomes hospital's "bottleneck". 


\section{Proof of Proposition B2}

For the LOS distribution (B44), all patients who underwent the surgical procedures on days $t-$ $\ell_{1}, \ldots, t$ will be still at the hospital on day $t$, whereas patients whose procedures were performed on any of the days $t-\ell_{2}, \ldots, t-\ell_{1}-1$ will remain in the hospital with probability $1-q$. Let $Y$ be the number of days from the set $\left\{t-\ell_{2}, \ldots, t-\ell_{1}-1\right\}$ whose patients remain in the hospital on day $t$. Under our assumptions, $Y$ is a binomial random variable with parameters $\left(\ell_{2}-\ell_{1}, 1-q\right)$.

The hospital expected daily profit function is given by:

$$
\Pi\left(a^{e}\right)=R\left(a^{e}+a^{u}\right)-\pi^{S} E\left[D_{t}\left(a^{e}, a^{u}\right)\right]-\pi^{N} E\left[O_{t}\left(a^{e}, a^{u}\right)\right],
$$

where we have slightly modified the notation for the total bed occupancy to account for the same number of urgent procedures performed on all days, and

$$
\begin{aligned}
& E\left[D_{t}\left(a^{e}, a^{u}\right)\right]=(1-p)\left(\left(a^{e}+a^{u}\right) s_{2}-\tau\right)^{+}+p\left(\left(a^{e}+a^{u}\right) s_{1}-\tau\right)^{+}, \\
& E\left[O_{t}\left(a^{e}, a^{u}\right)\right]=\sum_{y=0}^{\ell_{2}-\ell_{1}}\left(\begin{array}{c}
\ell_{2}-\ell_{1} \\
y
\end{array}\right)(1-q)^{y} q^{\ell_{2}-\ell_{1}-y}\left(\left(a^{e}+a^{u}\right)\left(\ell_{1}+y\right)-c\right)^{+},
\end{aligned}
$$

with $x^{+}=\max (x, 0)$.

a) Both $E\left[D_{t}\left(a^{e}, a^{u}\right)\right]$ and $E\left[O_{t}\left(a^{e}, a^{u}\right)\right]$ are piece-wise linear convex functions of $a^{e}$. Thus, (B56) is piece-wise linear concave in $a^{e}$. Consider the derivative of $\Pi\left(a^{e}\right)$ with respect to $a^{e}$ :

$$
\begin{aligned}
\frac{d \Pi}{d a^{e}} & =R-\pi^{S}\left((1-p) s_{2} \eta\left(a s_{2}-\tau\right)+p s_{1} \eta\left(a s_{1}-\tau\right)\right) \\
& -\pi^{N} \sum_{y=0}^{\ell_{2}-\ell_{1}}\left(\begin{array}{c}
\ell_{2}-\ell_{1} \\
y
\end{array}\right)(1-q)^{y-\ell_{1}} q^{\ell_{2}-\ell_{1}-y}\left(\ell_{1}+y\right) \eta\left(a\left(\ell_{1}+y\right)-c\right),
\end{aligned}
$$

where $a=a^{e}+a^{u}$. It is a piece-wise constant, non-increasing function of $a^{e}$ with discontinuities at the values of $a^{e}=a-a^{u}$ with $a \in I_{a}$. Then, (B51) establishes that $\Pi\left(a^{e}\right)$ has a unique maximum since it ensures that $\frac{d \Pi}{d a^{e}}$ cannot be equal to zero for any $a^{e}=a-a^{u}$ with $a \in I_{a}$.

b) As $a^{e}$ goes to infinity, all $\eta(\cdot)$ expressions on the right-hand side of (B59) become 1, and the right-hand side of (B59) is equal to:

$$
R-\pi^{S}\left(p s_{1}+(1-p) s_{2}\right)-\pi^{N}\left(q \ell_{1}+(1-q) \ell_{2}\right) .
$$

Then, for $R>\pi^{S}\left(p s_{1}+(1-p) s_{2}\right)-\pi^{N}\left(q \ell_{1}+(1-q) \ell_{2}\right)$, or $\frac{\pi^{S}}{r_{S}}+\frac{\pi^{N}}{r_{N}}<1$, the hospital would like to have as many surgical procedures as possible so that the optimal number of elective procedures is set to the upper bound on the demand, $U$.

c) In this part, we first consider the case without the demand constraint. The expected daily profit is concave in $a^{e}$, and its first derivative with respect to $a=a^{e}+a^{u}$ is a decreasing, piecewise-constant function of $a$ with discontinuities at the following $\ell_{2}-\ell_{1}+3$ points: $\frac{\tau}{s_{2}}, \frac{\tau}{s_{1}}$, and $\frac{c}{\ell_{2}}$, 
$\frac{c}{\ell_{2}-1}, \ldots, \frac{c}{\ell_{1}}$. Under conditions of the Proposition, the optimal value of $a$ exists, is unique, and is achieved at one of these points. Define, for $i=1,2$,

$$
k_{i}^{*}= \begin{cases}0, & \text { if } \frac{\tau}{s_{i}}<\frac{c}{\ell_{2}}, \\ \left(k \in\left\{1, \ldots, \ell_{2}-\ell_{1}\right\} \mid \frac{c}{\ell_{2}-k+1} \leq \frac{\tau}{s_{i}}<\frac{c}{\ell_{2}-k}\right), & \text { if } \frac{c}{\ell_{2}} \leq \frac{\tau}{s_{i}}<\frac{c}{\ell_{1}}, \\ \ell_{2}-\ell_{1}+1, & \text { if } \frac{\tau}{s_{i}} \geq \frac{c}{\ell_{1}} .\end{cases}
$$

Note that we can express $k_{i}^{*}$ as

$$
k_{i}^{*}=\left[\left(\ell_{2}-\ell_{1}+1-\left(\left(\frac{c}{\tau}\right) s_{i}-\ell_{1}\right)^{+}\right)^{+}\right],
$$

where $[x]$ is the integer part of $x$, and $x^{+}$is $\max (x, 0)$. Note that $k_{1}^{*} \geq k_{2}^{*}$.

Suppose first that the optimal value of $a$ is $\hat{a}=\frac{\tau}{s_{2}}$. Then, $\hat{a}=\frac{\tau}{s_{2}}$ is optimal if and only if

$$
\begin{gathered}
\pi^{N} \sum_{y=\ell_{2}-\ell_{1}+1-k_{2}^{*}}^{\ell_{2}-\ell_{1}}\left(\begin{array}{c}
\ell_{2}-\ell_{1} \\
y
\end{array}\right)(1-q)^{y-\ell_{1}} q^{\ell_{2}-\ell_{1}-y}\left(\ell_{1}+y\right)<R \\
<\pi^{S}(1-p) s_{2}+\pi^{N} \sum_{y=\ell_{2}-\ell_{1}+1-k_{2}^{*}}^{\ell_{2}-\ell_{1}}\left(\begin{array}{c}
\ell_{2}-\ell_{1} \\
y
\end{array}\right)(1-q)^{y-\ell_{1}} q^{\ell_{2}-\ell_{1}-y}\left(\ell_{1}+y\right) .
\end{gathered}
$$

Defining

$$
\Phi_{B}(v)= \begin{cases}1, & \text { if } v<0, \\
\sum_{y=v}^{\ell_{2}-\ell_{1}}\left(\begin{array}{c}
\ell_{2}-\ell_{1} \\
y
\end{array}\right)(1-q)^{y-\ell_{1}} q^{\ell_{2}-\ell_{1}-y}\left(\frac{\ell_{1}+y}{q \ell_{1}+(1-q) \ell_{2}}\right), & \text { if } 0 \leq v \leq \ell_{2}-\ell_{1}, \\
0, & \text { if } v>\ell_{2}-\ell_{1},\end{cases}
$$

we can express (B62) as

$$
\begin{aligned}
& \left(\frac{\pi^{N}}{r_{N}}\right) \Phi_{B}\left(\ell_{2}-\ell_{1}+1-k_{2}^{*}\right)<1 \\
< & \frac{\pi^{S}}{r_{S}}\left(\frac{(1-p) s_{2}}{p s_{1}+(1-p) s_{2}}\right)+\left(\frac{\pi^{N}}{r_{N}}\right) \Phi_{B}\left(\ell_{2}-\ell_{1}+1-k_{2}^{*}\right) .
\end{aligned}
$$

In a similar fashion, we can show that the optimal value of $a$ is $\hat{a}=\frac{\tau}{s_{1}}$ if and only if

$$
\begin{aligned}
& \left(\frac{\pi^{N}}{r_{N}}\right) \Phi_{B}\left(\ell_{2}-\ell_{1}+1-k_{1}^{*}\right)<1 \\
< & \frac{\pi^{S}}{r_{S}}\left(\frac{(1-p) s_{2}}{p s_{1}+(1-p) s_{2}}\right)+\left(\frac{\pi^{N}}{r_{N}}\right) \Phi_{B}\left(\ell_{2}-\ell_{1}+1-k_{1}^{*}\right) .
\end{aligned}
$$

Since the optimum of $\Pi(\hat{a})$ is unique, the intervals of values of $R$ described by (B63) and (B64) are non-overlapping for $s_{2}>s_{1}$. Algebraically, this follows from $k_{1}^{*} \geq k_{2}^{*}$.

Now, suppose that the optimum of the expected daily profit is reached at $\hat{a}=\frac{c}{\ell_{2}-m^{*}}, m^{*} \in$ $\left\{0,1, \ldots, \ell_{2}-\ell_{1}\right\}$. We need to consider three possible cases: a) $\frac{c}{\ell_{2}-m^{*}}<\frac{\tau}{s_{2}}$, b) $\frac{\tau}{s_{2}}<\frac{c}{\ell_{2}-m^{*}}<\frac{\tau}{s_{1}}$, and c) $\frac{\tau}{s_{1}}<\frac{c}{\ell_{2}-m^{*}}$. In case a), we have

$$
m^{*}<\ell_{2}-\left(\frac{c}{\tau}\right) s_{2}
$$


and $\hat{a}=\frac{c}{\ell_{2}-m^{*}}$ is optimal if and only if

$$
\begin{aligned}
& \pi^{N} \sum_{y=\ell_{2}-\ell_{1}-m^{*}+1}^{\ell_{2}-\ell_{1}}\left(\begin{array}{c}
\ell_{2}-\ell_{1} \\
y
\end{array}\right)(1-q)^{y-\ell_{1}} q^{\ell_{2}-\ell_{1}-y}\left(\ell_{1}+y\right) \leq R \\
&<\pi^{N} \sum_{y=\ell_{2}-\ell_{1}-m^{*}}^{\ell_{2}-\ell_{1}}\left(\begin{array}{c}
\ell_{2}-\ell_{1} \\
y
\end{array}\right)(1-q)^{y-\ell_{1}} q^{\ell_{2}-\ell_{1}-y}\left(\ell_{1}+y\right),
\end{aligned}
$$

or, equivalently,

$$
\begin{aligned}
& \left(\frac{\pi^{N}}{r_{N}}\right) \Phi_{B}\left(\ell_{2}-\ell_{1}+1-m^{*}\right)<1 \\
< & \pi^{N}\left(\frac{\pi^{N}}{r_{N}}\right) \Phi_{B}\left(\ell_{2}-\ell_{1}-m^{*}\right) .
\end{aligned}
$$

In case b), we have

$$
\ell_{2}-\left(\frac{c}{\tau}\right) s_{2}<m^{*}<\ell_{2}-\left(\frac{c}{\tau}\right) s_{1},
$$

and $\hat{a}=\frac{c}{\ell_{2}-m^{*}}$ is optimal if and only if

$$
\begin{aligned}
& \frac{\pi^{S}}{r_{S}}\left(\frac{(1-p) s_{2}}{p s_{1}+(1-p) s_{2}}\right)+\left(\frac{\pi^{N}}{r_{N}}\right) \Phi_{B}\left(\ell_{2}-\ell_{1}+1-m^{*}\right)<1 \\
< & \frac{\pi^{S}}{r_{S}}\left(\frac{(1-p) s_{2}}{p s_{1}+(1-p) s_{2}}\right)+\left(\frac{\pi^{N}}{r_{N}}\right) \Phi_{B}\left(\ell_{2}-\ell_{1}-m^{*}\right) .
\end{aligned}
$$

Finally, in case c), we have

$$
\ell_{2}-\left(\frac{c}{\tau}\right) s_{1}<m^{*}
$$

and $\hat{a}=\frac{c}{\ell_{2}-m^{*}}$ is optimal if and only if

$$
\begin{aligned}
& \frac{\pi^{S}}{r_{S}}+\left(\frac{\pi^{N}}{r_{N}}\right) \Phi_{B}\left(\ell_{2}-\ell_{1}+1-m^{*}\right)<1 \\
< & \frac{\pi^{S}}{r_{S}}+\left(\frac{\pi^{N}}{r_{N}}\right) \Phi_{B}\left(\ell_{2}-\ell_{1}-m^{*}\right) .
\end{aligned}
$$

Comparing (B65), (B68), and (B70), we observe that the intervals of values of $R$ described by (B67), (B69), and (B71) cannot overlap, and each of the conditions (B67), (B69), and (B71) can hold for at most one value of $m^{*}$, consistent with the uniqueness of the optimum of the hospital expected daily profit function. Hence, $\hat{a}^{e}=\hat{a}-a^{e}$, when there is no demand constraint.

When the demand is bounded above by $U$, the optimal number of elective procedures is given by $\hat{a}^{e}=\min \left\{\hat{a}-a^{e}, U\right\}$. 


\section{Appendix C: Additional Numerical Results}

\section{C.1. Data}

Table C1 shows the relative contributions of the top ten procedures to the overall portfolio of surgeries performed at the hospital during this time period. As the percentages in Table $\mathrm{C} 1$ indicate, the portfolio of the surgical procedures for the hospital we study is not overly concentrated on any particular procedure: the cumulative share of the top ten procedures is $36.91 \%$.

\begin{tabular}{|c|c|}
\hline Procedure & $\begin{array}{c}\text { Percentage of the Total } \\
\text { Number of Procedures }\end{array}$ \\
\hline 1 & 7.17 \\
\hline 2 & 5.73 \\
\hline 3 & 4.38 \\
\hline 4 & 3.96 \\
\hline 5 & 3.37 \\
\hline 6 & 3.07 \\
\hline 7 & 2.38 \\
\hline 8 & 2.35 \\
\hline 9 & 2.26 \\
\hline 10 & 2.24 \\
\hline
\end{tabular}

Table C1 Ten most frequent surgical procedures.

\section{C.2. "Base Case" Estimation}

In our numerical study we use the "base case" setting defined as follows. We set the OR capacity value $\tau$ and recovery bed capacity value $c$ to ensure that the current portfolio of three procedures results in the expected utilization levels of OR time and recovery beds that match the estimates for the respective average utilization levels 0.7022 and 0.7298 , respectively, in the hospital we study. In other words, using the data from Table 1, we require

$$
3.20(1.07+0.17)+4.38(0.77+0.18)+2.90(0.75+0.03)=0.7022 \tau,
$$

and

$$
4.31(1.07+0.17)+5.80(0.77+0.18)+2.55(0.75+0.03)=0.7298 c,
$$

so that $\tau=14.79$ hours and $c=17.60$ beds, but we round the bed capacity to the closest integer and use $c=18.00$ for the base case.

The final two parameters we use for the "base case" setting are the penalty cost values $\pi^{N}$ and $\pi^{S}$ associated with exceeding the nominal bed and OR capacities, respectively. We estimate these cost parameters using a simple method of minimizing the aforementioned sum of squared percentage deviations. We need to do this because we do not observe the cost parameters, $\pi^{N}$ and 
$\pi^{S}$, in the data we collected. The estimation is done via a structural approach which assumes that the decision maker (the hospital administrators) is rational, knows the problem parameters (e.g., LOS distributions, cost parameters), and selects a portfolio of elective procedures that maximizes the hospitals objective function (profit). These assumptions are standard in the existing literature; see, for example, Cohen et al. (2003), Olivares et al. (2008), and Deshpande and Arıkan (2012). Regarding the particular assumption that the hospital is profit maximizing, there is strong empirical evidence that hospitals (both for-profit and not-for-profit) can be treated as profit maximizing entities that are aware of their operating costs (Duggan 2000, Brickley and Van Horn 2002).

We select the values of $\pi^{N}$ and $\pi^{S}$ such that the resulting optimal portfolio of elective procedures provides as close as possible a match for the current portfolio as well as the current utilization values achieved at $\tau=14.79$ and $c=18.00$. We measure the degree of such a match using the sum of the squared percentage deviations between the current portfolio components (numbers of elective procedures for each procedure type); the current capacity utilization levels; and the portfolio components and capacity utilization levels optimal under a particular combination of the penalty cost values.

To find these "best-fit" $\pi^{N}$ and $\pi^{S}$ values for our base case, we perform a grid search with the objective of minimizing the aforementioned sum of squared percentage deviations. The range of $\pi^{N}$ and $\pi^{S}$ values in the grid search was 0 to 1,000 with a step size of 0.1 . For each pair of $\pi^{N}$ and $\pi^{S}$ values on the grid, we find the optimal portfolio that maximizes the expected daily profit in (27). We evaluate the integral in (27) using Monte Carlo simulation because the number of combinations of nonelective procedures is prohibitively large for the direct evaluation of the integrals. In particular, there are three procedures in our example, each with an LOS support of 20 days. Thus, each integral evaluationsec-num2 depends on the number of nonelective arrivals in the past 20 days for each procedure. To simplify the problem further, consider the case in which the number of nonelective cases for each procedure on each day is either 0 or 1 with probabilities reported in column 3 of Table 1 . In such a problem, the number of possible combinations in the integral is $2^{3 \times 20}=2^{60}$. Our Monte Carlo simulation evaluates the integral by randomly drawing the values for the daily numbers of nonelective procedures using $n=1,000$ simulation runs.

Because the resulting best-fit values of $\pi^{N}$ and $\pi^{S}$ depend on the random seed of the Monte Carlo simulation, we perform the grid search using 10 different random seeds. The subsequent average best-fit penalty cost values are $\pi^{N}=14.03$ and $\pi^{S}=0.55$. Note that both values are expressed as multiples of the hospital charges for procedure 2. In what follows we use $\pi_{b}^{N}$ and $\pi_{b}^{S}$ to denote these "base-case" penalty cost values. 
Percent Profit Difference:

Exact vs. Approximated

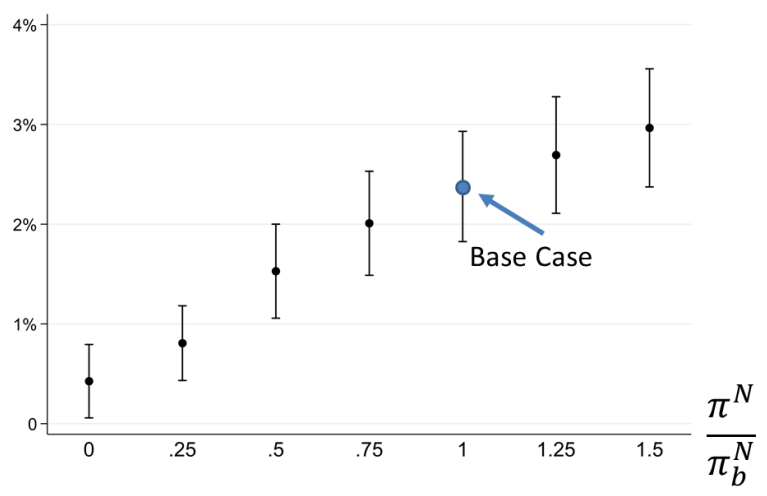

Percent Profit Difference:

Exact vs. Approximated

b)

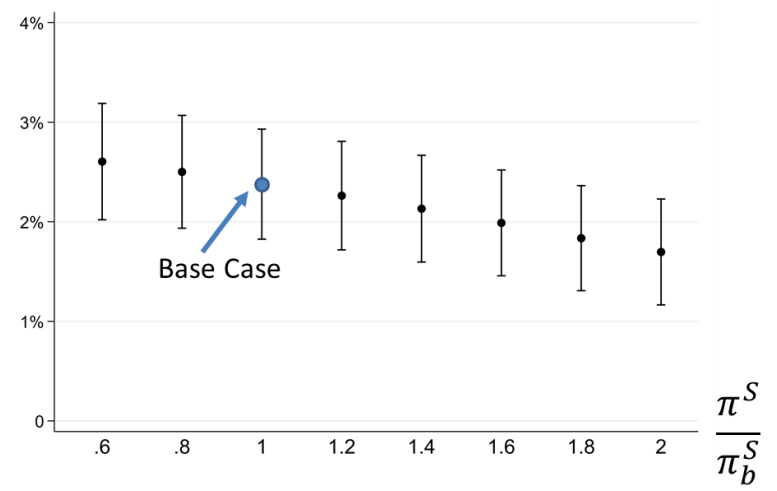

Figure C1 : Percentage difference between the exact and approximated values of the optimal profit as a function of a) the penalty cost $\pi^{N}$ associated with exceeding the nominal bed capacity $c$, and b) the penalty cost $\pi^{S}$ associated with exceeding the nominal OR capacity $\tau$. The bars show the simulation-based $95 \%$ confidence intervals.

\section{C.3. The Impact of Penalty Costs, $\pi^{N}$ and $\pi^{S}$, on the Accuracy of the CLT-based Approximation}

We investigate the impact of the penalty $\operatorname{costs}, \pi^{N}$ and $\pi^{S}$, on the performance of our approximation in Figure C1. To compare the exact and the approximate approaches, we used 20 different random seeds for each value of $\pi^{N}$ and $\pi^{S}$. We observe that as the values of $\pi^{N}$ and $\pi^{S}$ change by $50 \%$ in either direction, the approximated optimal profit stays within $5 \%$ of its exact value.

In addition, in Figure C2, we examine how changing the value of $\pi^{N}$ impacts the difference between the exact and approximated optimal values of each procedure in the portfolio. As the value of $\pi^{N}$ increases, the approximated portfolio favors procedure 2 at the expense of procedures 1 and 3. The differences are, however, in a reasonable range. The only exception is when $\pi^{N}$ approaches zero in Figure C2c, but this seemingly large difference is due to procedure 3 having a near-zero share in the approximate and exact portfolios for small values $\pi^{N}$. Note that for $\pi^{N}=0$, the optimal value of procedure 3 in the approximated solution is $\hat{a}_{3}^{e}=4.57 \times 10^{-7}$, which is almost 0 , and the optimal value of procedure 3 in the exact solution is also small, $\hat{a}_{3, E}^{e}=6.04 \times 10^{-4}$. This leads to the percentage difference between the two values to be about $100 \%\left(\frac{\hat{a}_{3, E}^{e}-\hat{a}_{3}^{e}}{\hat{a}_{3, E}^{e}} \times 100 \approx 100 \%\right)$, but both $\hat{a}_{3}^{e}$ and $\hat{a}_{3, E}^{e}$ are practically negligible compared to the other two procedures in the portfolio, $\hat{a}_{1, E}^{e}=3.16$ and $\hat{a}_{2, E}^{e}=0.88$.

In a similar fashion, Figure C3 examines how changes in the value of $\pi^{S}$ impact the difference between the exact and approximated optimal values of each procedure in the portfolio. We observe minor changes in the difference as a function of $\pi^{S}$ for all three procedures.

Based on our observations in Figures C1-C3, and the results in Figure 7, we conclude that the approximation performs well. 
Percent Difference, Procedure 1:

Exact vs. Approximated

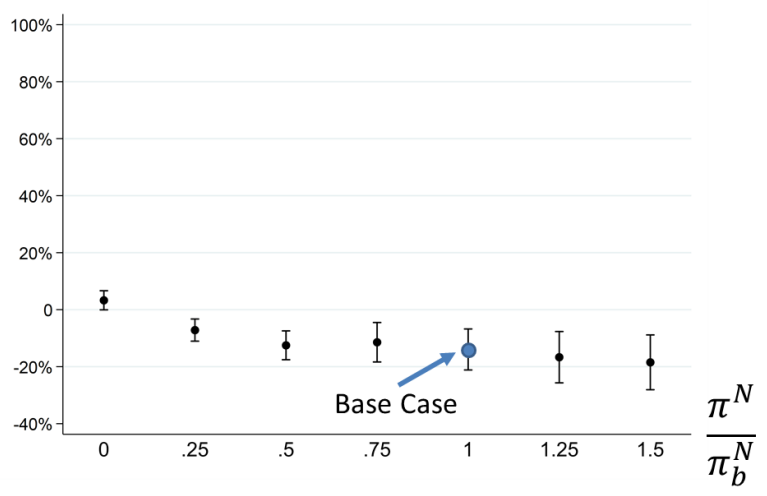

Percent Difference, Procedure 2:

Exact vs. Approximated

b)

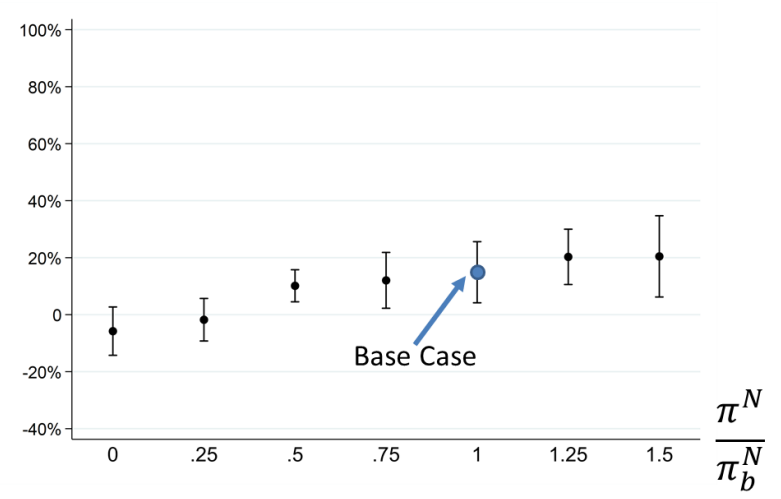

Percent Difference, Procedure 3:

Exact vs. Approximated

c)

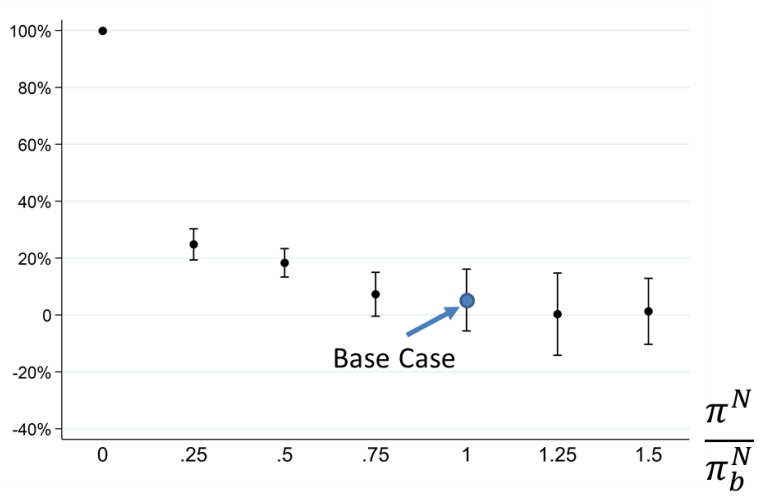

Figure C2 : Percentage difference between the exact and approximated values of each procedure in the optimal portfolio as a function of the penalty $\operatorname{cost} \pi^{N}$ associated with exceeding the nominal bed capacity $c$. The bars show the simulation-based $95 \%$ confidence intervals. 
Percent Difference, Procedure 1:

Exact vs. Approximated

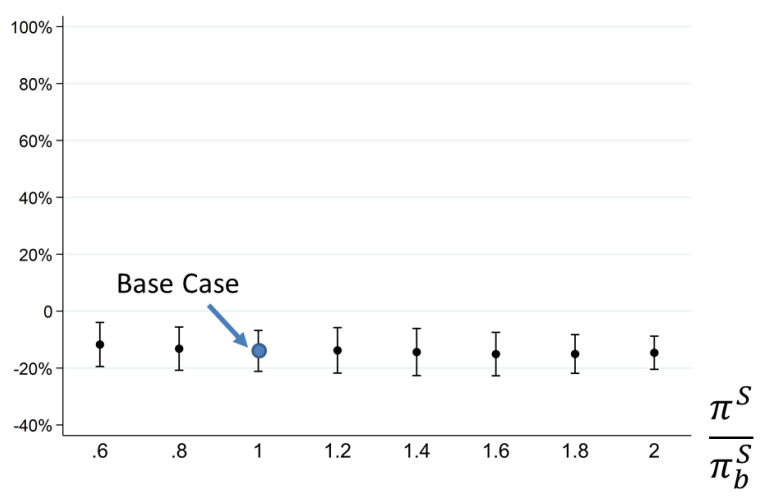

Percent Difference, Procedure 2:

Exact vs. Approximated

b)

Percent Difference, Procedure 3:

Exact vs. Approximated
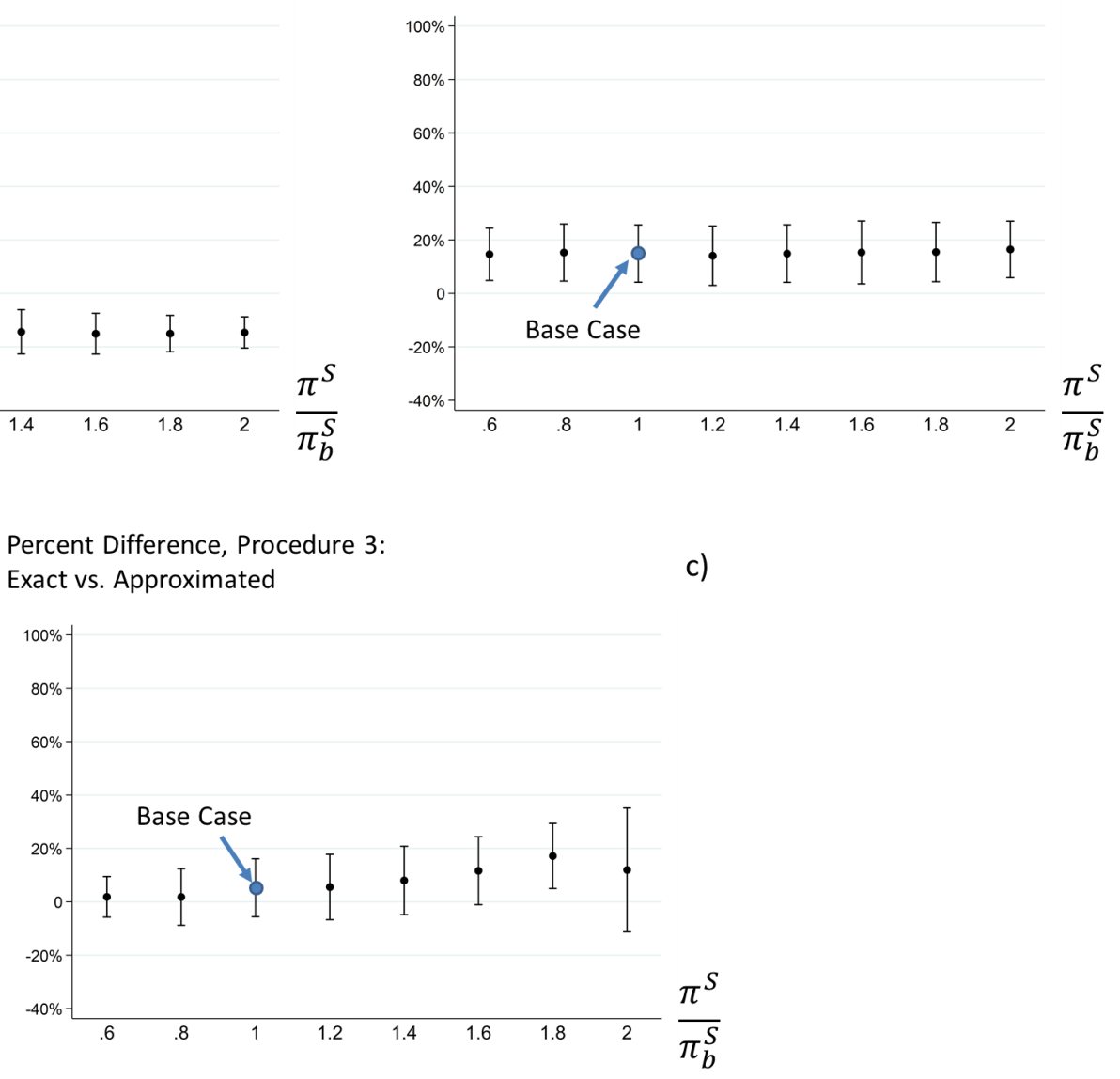

Figure C3 : Percentage difference between the exact and approximated values of each procedure in the optimal portfolio as a function of the penalty $\operatorname{cost} \pi^{S}$ associated with exceeding the nominal OR capacity $\tau$. The bars show the simulation-based $95 \%$ confidence intervals. 


\section{C.4. Figures for Section 6.3}

In Figures $\mathrm{C} 6$ and $\mathrm{C} 7$, we illustrate the impact of varying the parameters of the distributions of OR time and bed occupancy for procedure 1. To study the impact of changes in the variance of OR time, we maximize (27) for different values of $\left(\sigma_{1}^{S}\right)^{2}$. This is feasible because $\left(\sigma_{1}^{S}\right)^{2}$ can be changed without changing the mean OR time, $\mu_{1}^{S}$. For bed occupancy, however, both $\mu_{1}^{N}$ and $\left(\sigma_{1}^{N}\right)^{2}$ are functions of $p_{1, T}^{L}, T=0, \cdots, L_{\max }^{1}$. Therefore, we alter the distribution of bed occupancy by solving the following constrained optimization problem. The objective function is to keep the overall shape of the new bed occupancy distribution "close" to the current one for procedure 1 by minimizing the sum of squared errors between the LOS pdf of the new distribution and the empirical LOS pdf for procedure 1. The two constraints are that the new LOS distribution (for which we use the subscript $1^{\prime}$ ) should have (1) the same mean, i.e., $\mu_{1^{\prime}}^{N}=\mu_{1}^{N}$, and (2) the "scaled" variance of bed occupancy, i.e., $\left(\sigma_{1^{\prime}}^{N}\right)^{2}=\kappa\left(\sigma_{1}^{N}\right)^{2}$, where $0.5 \leq \kappa \leq 1.5$ is the scaling parameter. 
Expected Bed Utilization

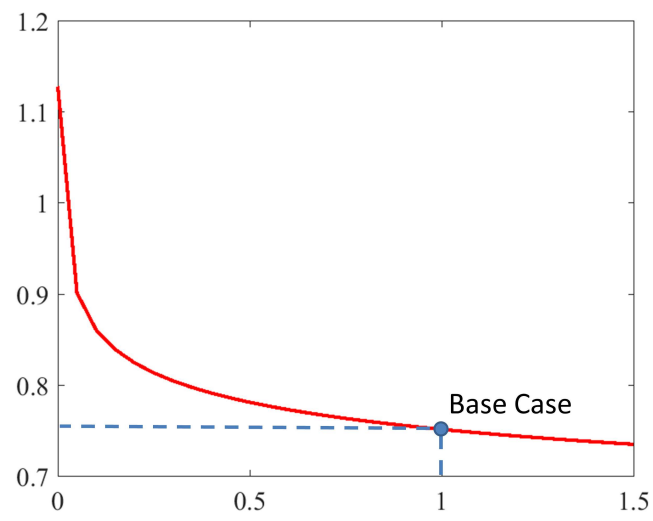

Components of Elective Bed Utilization

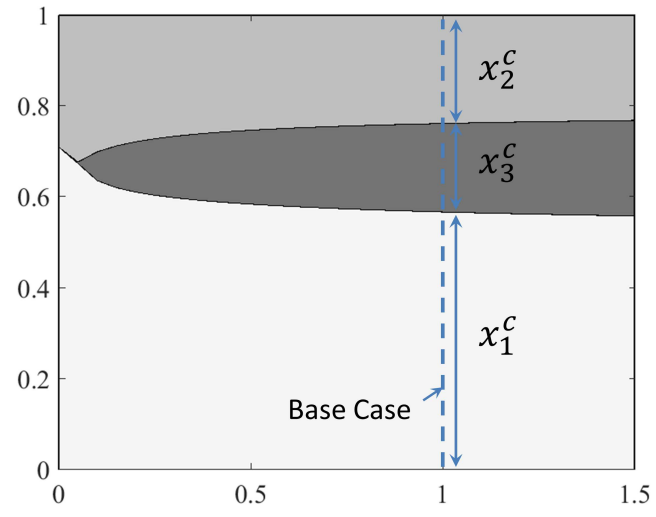

a) Expected OR Utilization

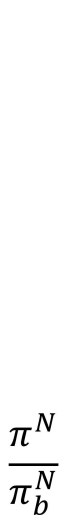

c)

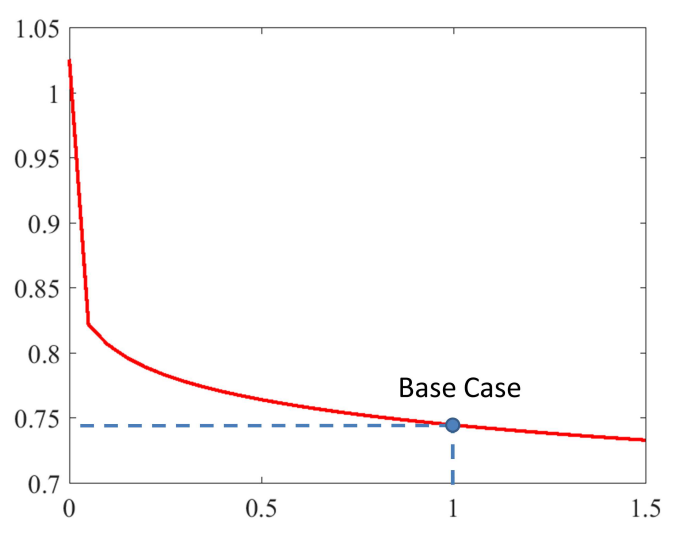

b)

Components of Elective OR Utilization

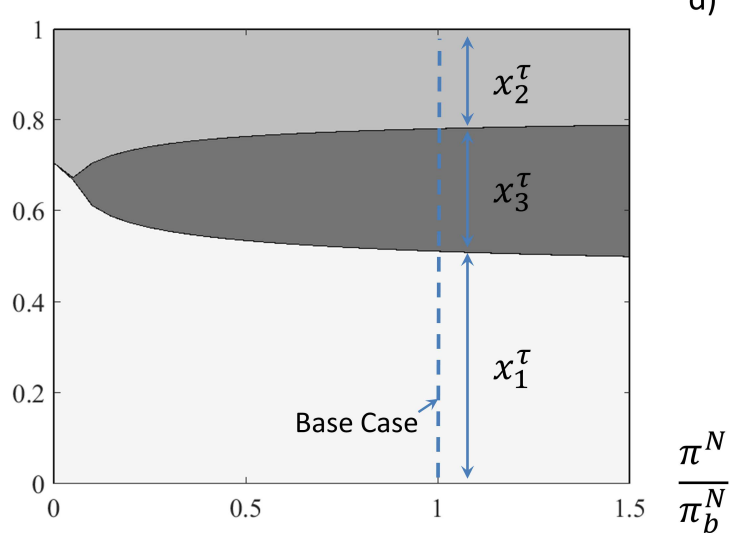

Figure C4: The impact of changes in the penalty $\operatorname{cost} \pi^{N}$ associated with exceeding the nominal bed capacity $c$ : a) expected bed utilization, $u_{c}$, b) expected OR utilization, $\left.u_{\tau}, \mathrm{c}\right)$ procedure components of the elective bed utilization, $\left(x_{1}^{c}, x_{2}^{c}, x_{3}^{c}\right)$, and d) procedure components of the elective OR utilization, $\left(x_{1}^{\tau}, x_{2}^{\tau}, x_{3}^{\tau}\right)$. 


\section{Expected Bed Utilization}

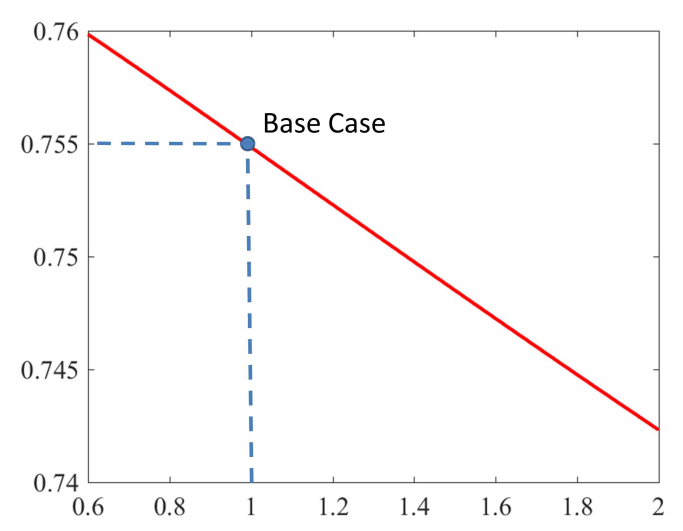

Components of Elective Bed Utilization

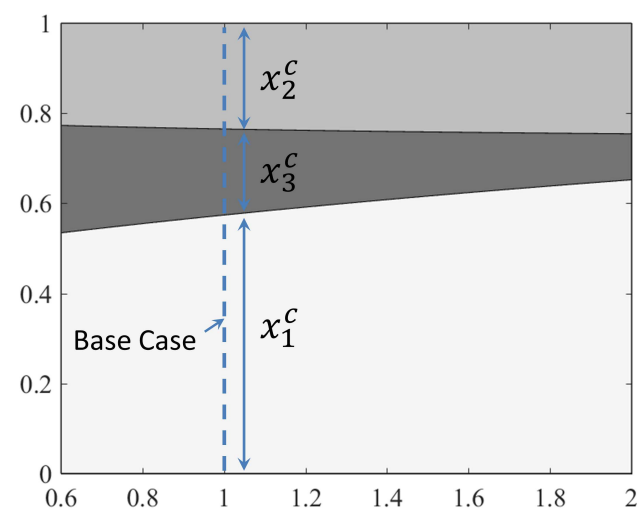

a)

\section{Expected OR Utilization}

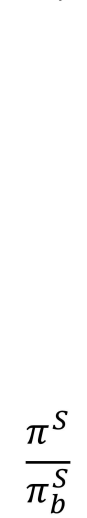

c)

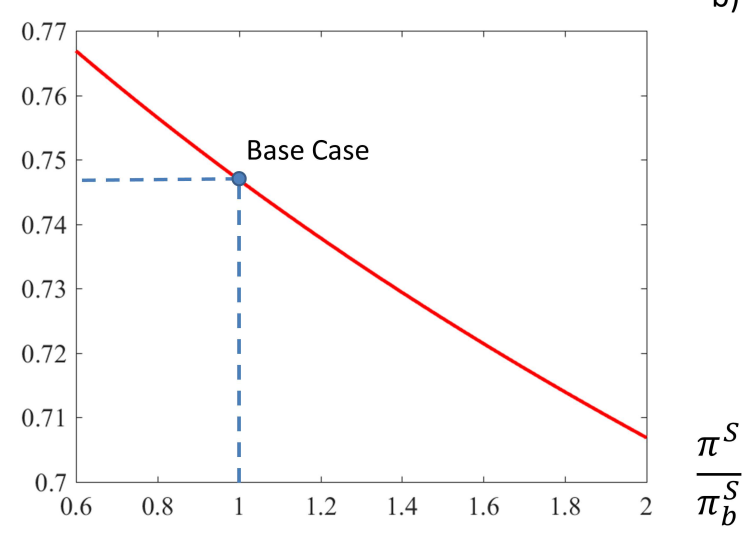

Components of Elective OR Utilization

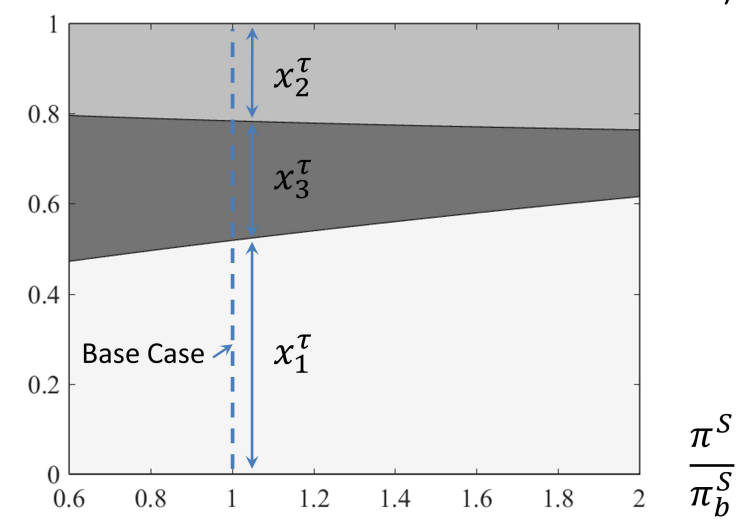

Figure C5 : The impact of changes in the penalty cost $\pi^{S}$ associated with exceeding the nominal OR capacity $\tau$ : a) expected bed utilization, $u_{c}$, b) expected OR utilization, $\left.u_{\tau}, \mathrm{c}\right)$ procedure components of the elective bed utilization, $\left(x_{1}^{c}, x_{2}^{c}, x_{3}^{c}\right)$, and d) procedure components of the elective OR utilization, $\left(x_{1}^{\tau}, x_{2}^{\tau}, x_{3}^{\tau}\right)$. 
Expected Bed Utilization

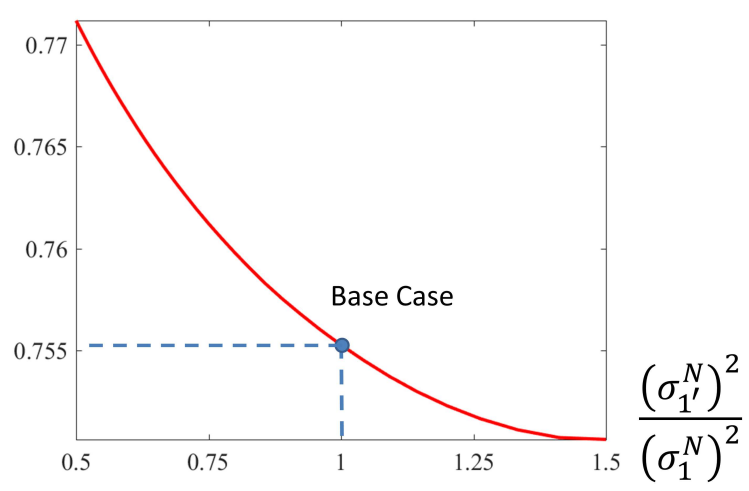

Components of Elective Bed Utilization

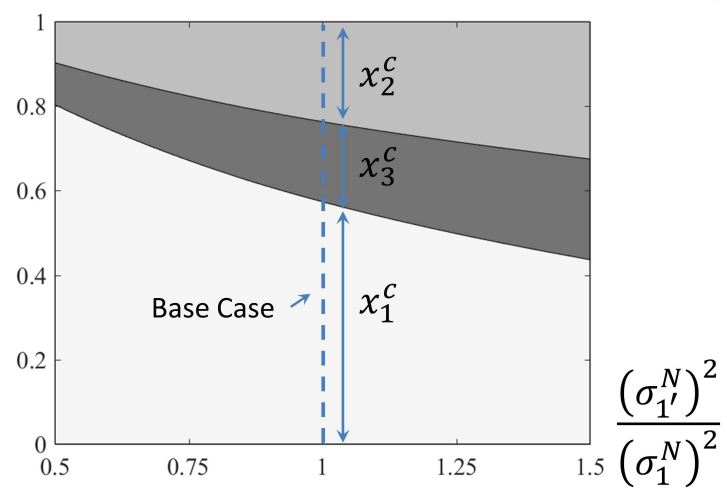

a)
Expected OR Utilization

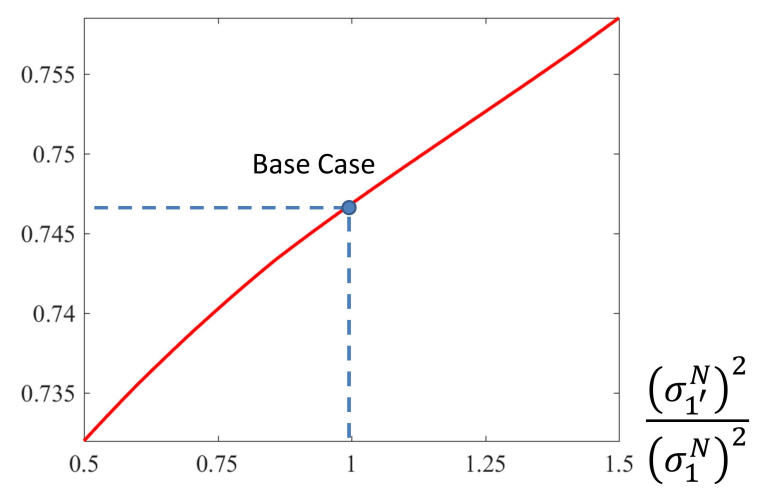

Components of Elective OR Utilization

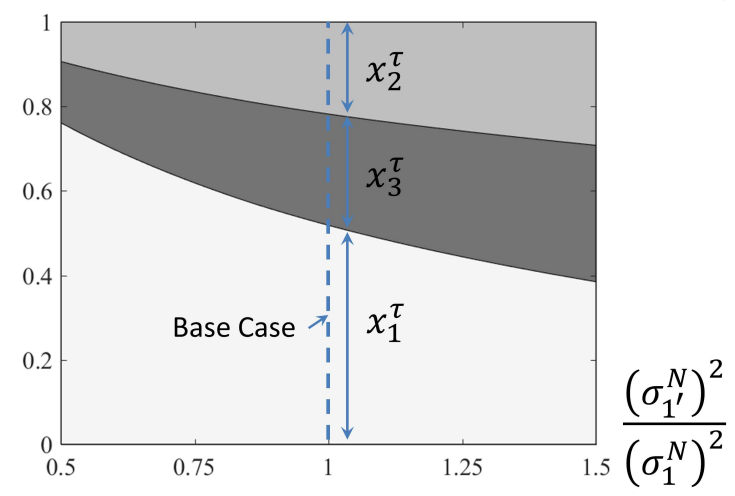

Figure C6 : The impact of changes in the variance of bed occupancy for procedure 1: a) expected bed utilization, $u_{c}$, b) expected OR utilization, $\left.u_{\tau}, \mathrm{c}\right)$ procedure components of the elective bed utilization, $\left(x_{1}^{c}, x_{2}^{c}, x_{3}^{c}\right)$, and $\mathrm{d})$ procedure components of the elective OR utilization, $\left(x_{1}^{\tau}, x_{2}^{\tau}, x_{3}^{\tau}\right)$. 
Expected Bed Utilization

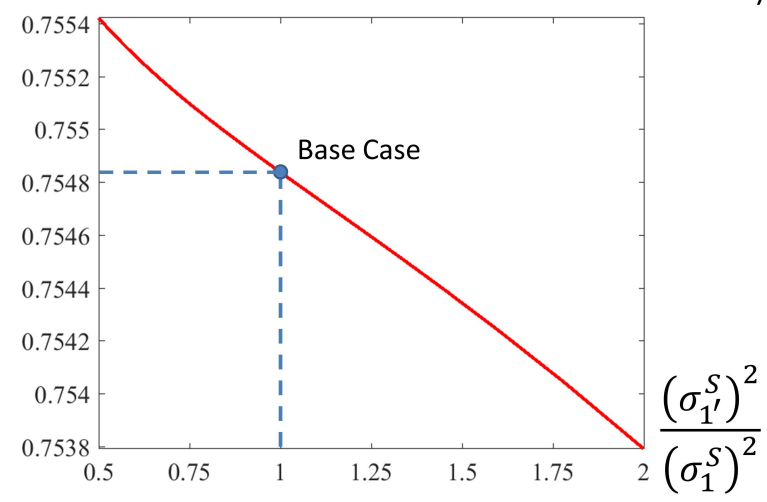

Components of Elective Bed Utilization

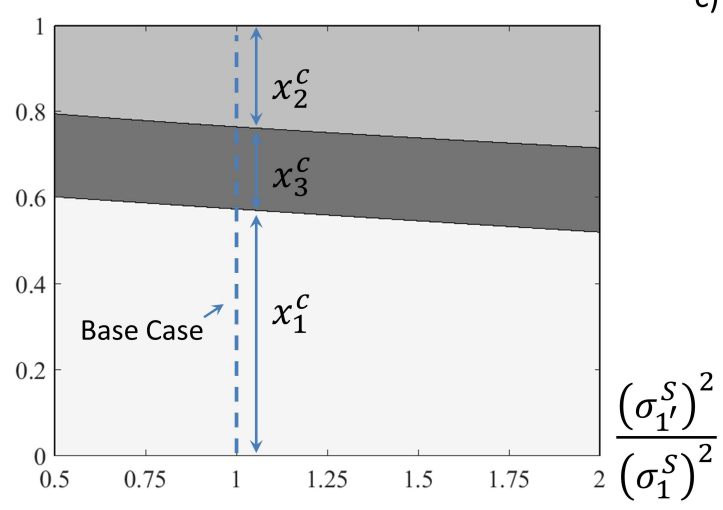

a)
Expected OR Utilization

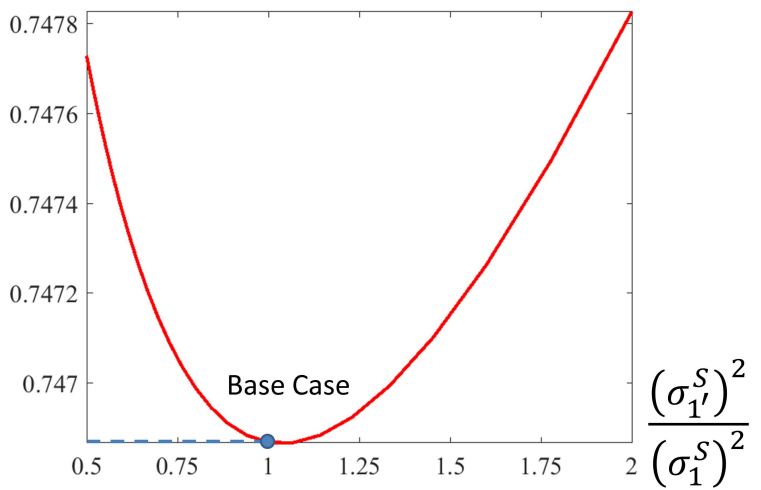

Components of Elective OR Utilization

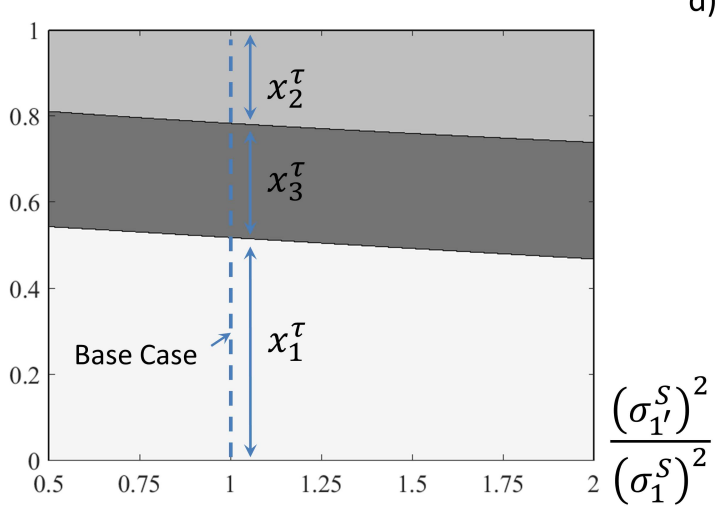

Figure C7 : The impact of changes in the variance of surgery duration for procedure 1: a) expected bed utilization, $\left.u_{c}, \mathrm{~b}\right)$ expected OR utilization, $\left.u_{\tau}, \mathrm{c}\right)$ procedure components of the elective bed utilization, $\left(x_{1}^{c}, x_{2}^{c}, x_{3}^{c}\right)$, and $\mathrm{d}$ ) procedure components of the elective OR utilization, $\left(x_{1}^{\tau}, x_{2}^{\tau}, x_{3}^{\tau}\right)$. 


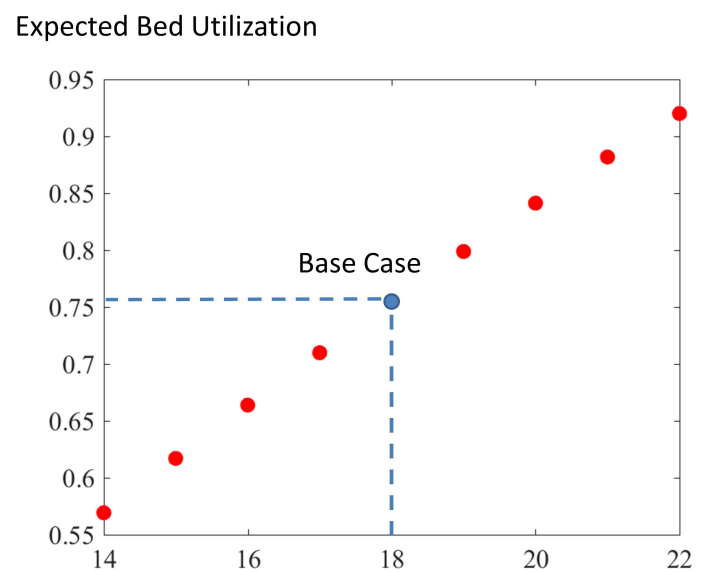

a)

Expected OR Utilization

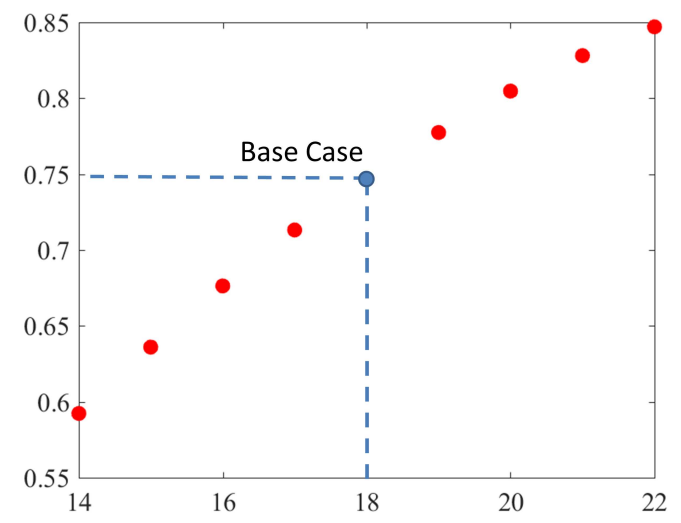

Components of Elective OR Utilization

c)

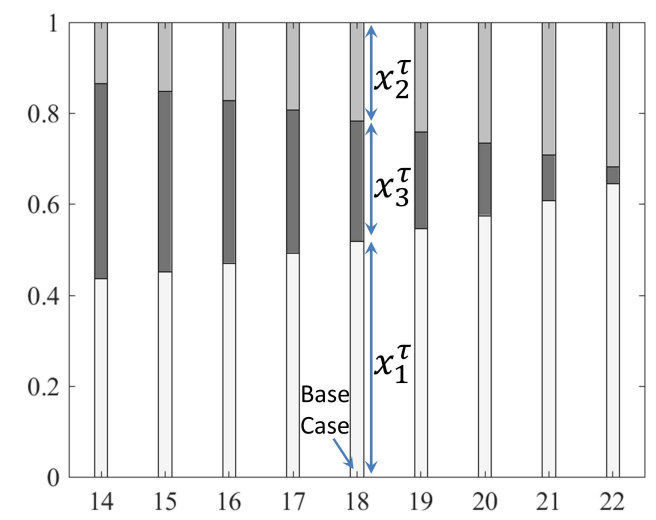

$C$ b)

$C$

d)

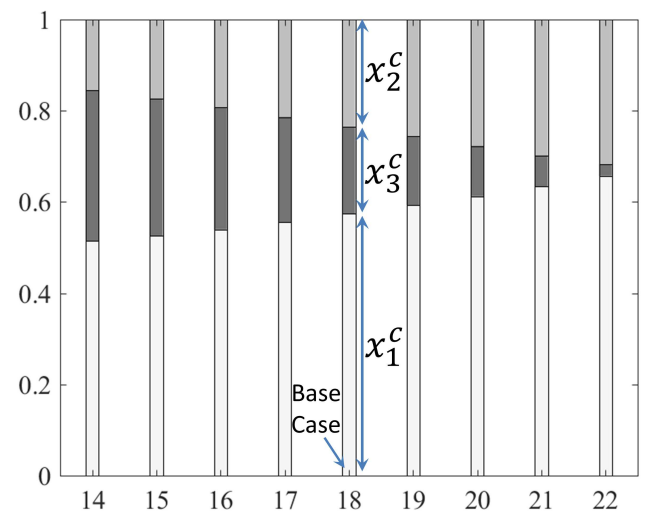

Figure C8 : The impact of changes in the hospital bed capacity: a) expected bed utilization, $u_{c}$, b) expected OR utilization, $\left.u_{\tau}, \mathrm{c}\right)$ procedure components of the elective bed utilization, $\left(x_{1}^{c}, x_{2}^{c}, x_{3}^{c}\right)$, and d) procedure components of the elective OR utilization, $\left(x_{1}^{\tau}, x_{2}^{\tau}, x_{3}^{\tau}\right)$. 
Performance Gap of the "Front-End" Approach, $\Delta_{S}$

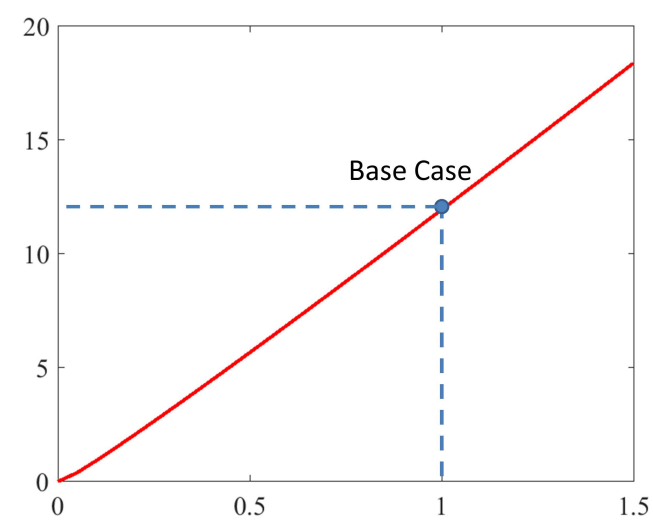

Performance Gap of the "Front-End" Approach, $\Delta_{S}$

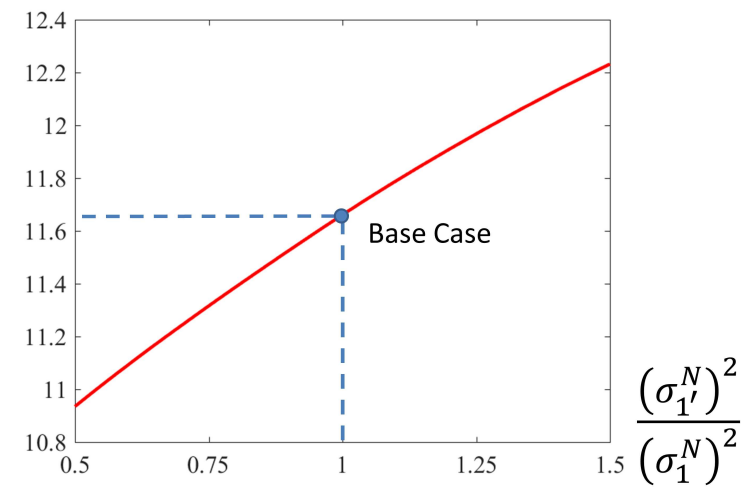

a)
Performance Gap of the "Front-End" Approach, $\Delta_{S}$

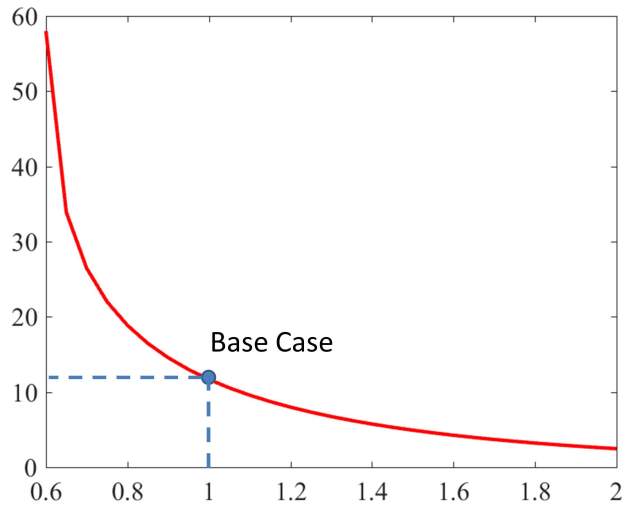

$\frac{\pi^{S}}{\pi_{b}^{S}}$

Performance Gap of the "Front-End" Approach, $\Delta_{S}$

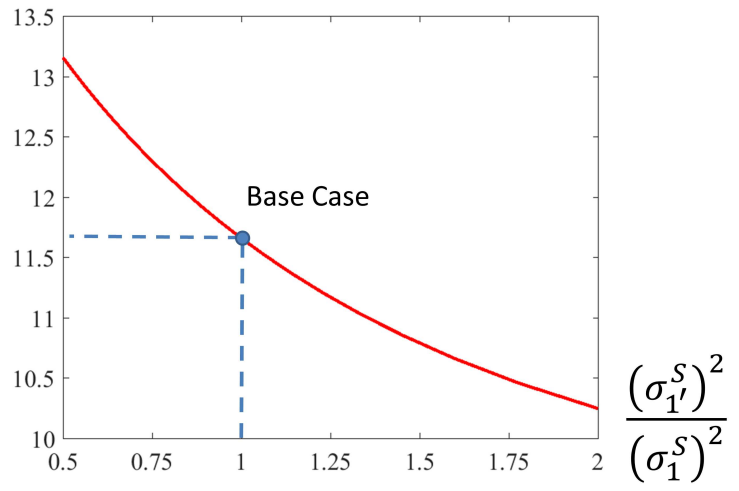

b)

d)

Performance Gap of the "Front-End" Approach, $\Delta_{S}$

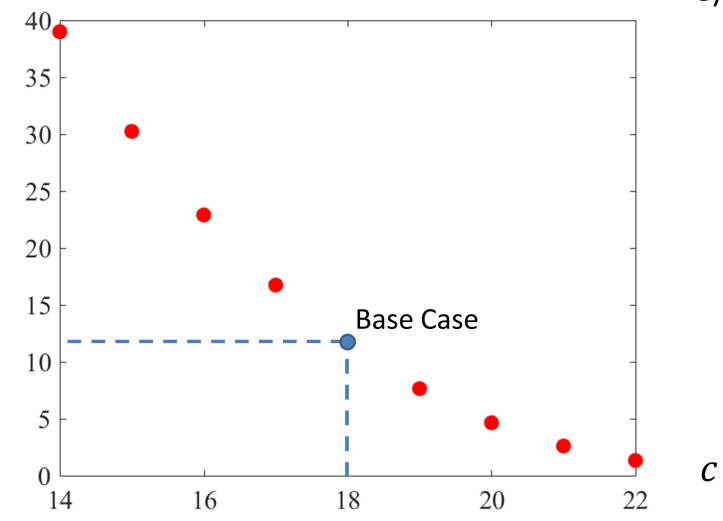

Figure C9 : The impact of changes in the $\pi^{N}, \pi^{S},\left(\sigma_{1}^{N}\right)^{2},\left(\sigma_{1}^{S}\right)^{2}$, and $c$ on the performance gap of the "front-end" approach, $\Delta_{S}$. 


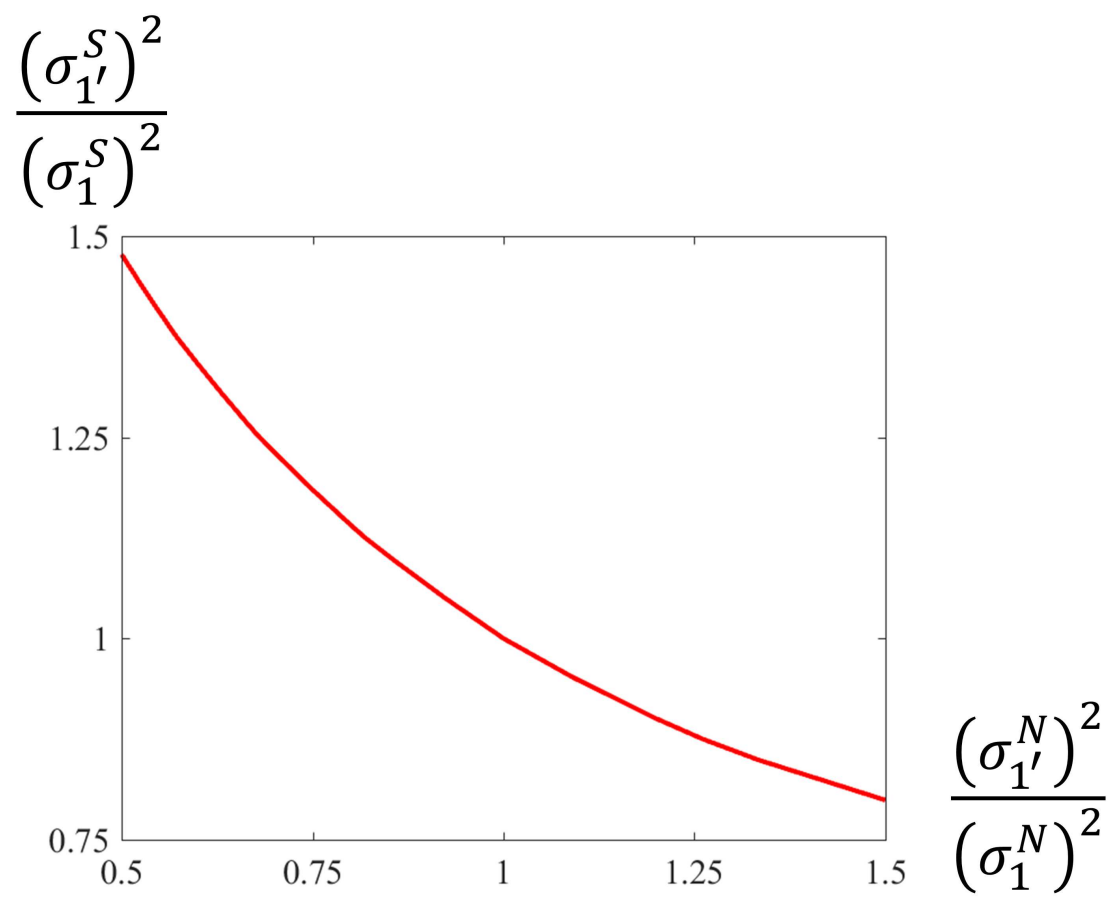

Figure C10: The bed occupancy and OR time variance levels that keep the front-end performance gap at the baseline value. 